\title{
Driver Speed and Acceleration Behaviour on Canadian Roads
}

\author{
Submitted by \\ Letian Yang, B. Eng.
}

A thesis submitted to the Faculty of Graduate Studies and Research in partial fulfillment of the requirements for the degree of Master of Applied Science

\author{
Department of Civil and Environmental Engineering \\ Carleton University \\ Ottawa, Ontario
}

(C) Letian Yang 2007

The Master of Applied Science in Civil Engineering Program is a joint program with the University of Ottawa, administrated by the Ottawa-Carleton Institute for Civil Engineering 


$\begin{array}{ll}\begin{array}{l}\text { Library and } \\ \text { Archives Canada }\end{array} & \begin{array}{l}\text { Bibliothèque et } \\ \text { Archives Canada }\end{array} \\ \begin{array}{l}\text { Published Heritage } \\ \text { Branch }\end{array} & \begin{array}{l}\text { Direction du } \\ \text { Patrimoine de l'édition }\end{array} \\ \begin{array}{l}\text { 395 Wellington Street } \\ \text { Ottawa ON K1A 0N4 }\end{array} & \begin{array}{l}\text { 395, rue Wellington } \\ \text { Ottawa ON K1A ON4 }\end{array} \\ \text { Canada } & \begin{array}{l}\text { Canada } \\ \end{array}\end{array}$

Yourfile Votre référence

ISBN: 978-0-494-36835-0

Ourfile Notre référence

ISBN: 978-0-494-36835-0

NOTICE:

The author has granted a nonexclusive license allowing Library and Archives Canada to reproduce, publish, archive, preserve, conserve, communicate to the public by telecommunication or on the Internet, loan, distribute and sell theses worldwide, for commercial or noncommercial purposes, in microform, paper, electronic and/or any other formats.

The author retains copyright ownership and moral rights in this thesis. Neither the thesis nor substantial extracts from it may be printed or otherwise reproduced without the author's permission.
AVIS:

L'auteur a accordé une licence non exclusive permettant à la Bibliothèque et Archives Canada de reproduire, publier, archiver, sauvegarder, conserver, transmettre au public par télécommunication ou par l'Internet, prêter, distribuer et vendre des thèses partout dans le monde, à des fins commerciales ou autres, sur support microforme, papier, électronique et/ou autres formats.

L'auteur conserve la propriété du droit d'auteur et des droits moraux qui protège cette thèse. $\mathrm{Ni}$ la thèse ni des extraits substantiels de celle-ci ne doivent être imprimés ou autrement reproduits sans son autorisation.
In compliance with the Canadian

Privacy Act some supporting forms may have been removed from this thesis.

While these forms may be included in the document page count, their removal does not represent any loss of content from the thesis.
Conformément à la loi canadienne sur la protection de la vie privée, quelques formulaires secondaires ont été enlevés de cette thèse.

Bien que ces formulaires aient inclus dans la pagination, il n'y aura aucun contenu manquant.

\section{Canadä}


This thesis is dedicated to:

My wife, Lei Guo 


\begin{abstract}
Driver speed and acceleration/deceleration behaviour studies are often used in highway facility design, vehicle fuel consumption and emission studies, and traffic safety programs. This study analyzed driver behaviour in terms of speed choice and acceleration and deceleration performances using experimental data collected from real road driving in the fall of 2005 . The selected test route covered the most common road classes including an urban freeway, two-lane rural highways, a rural freeway, and urban/suburban roads in Eastern Ontario. The test vehicle was equipped with a variety of instruments capable of measuring diverse operational data. Driver speed, acceleration, and deceleration behaviours on different road classes were analyzed under both free-flow and non-free-flow conditions based on the experimental data collected. Additionally, the drivers involved in this experiment were classified into three types based on their speed behaviour. The speed choice and acceleration/deceleration characteristics of the different driver types were studied and compared accordingly.
\end{abstract}




\section{ACKNOWLEDGEMENTS}

I would like to express my deepest gratitude to my supervisor, Professor Yasser Hassan, for his excellent guidance, encouragement, patience, and understanding during this research. This work could not have been completed without his invaluable input.

I would like to thank the experimenters, Dalia Said and Bin Nie, for their diligent efforts in collecting data. I would also like to express my thanks to the volunteer drivers and other participants for their help in the experiment. Without their selfless contributions, the field study would have not been accomplished and this research was certainly not possible.

I would like to thank Netistix Technologies Corporation for their generous supports in the installation of the Vehicle Interface Unit (VIU) for data collection, and for their extraction and provision of the VIU data. Financial support by Netistix Technologies Corporation is gratefully acknowledged as well. The equipment used in this research was acquired by funds provided by the Canada Foundation for Innovation (CFI) and the Ontario Trust Fund.

Finally, I want to express a special gratitude to my wife, Lei Guo, for her support, encouragement, love, and incredible help throughout my work. She always gave me strength when I tried new things. 


\section{TABLE OF CONTENTS}

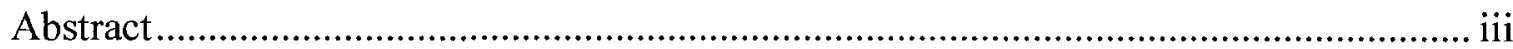

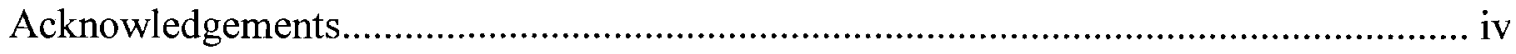

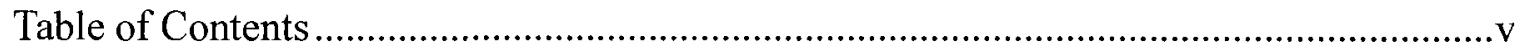

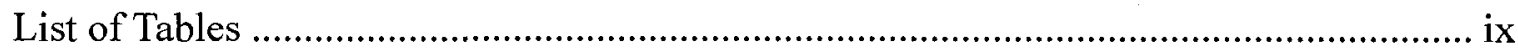

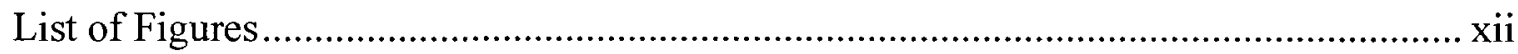

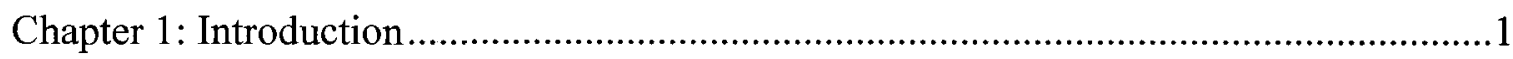

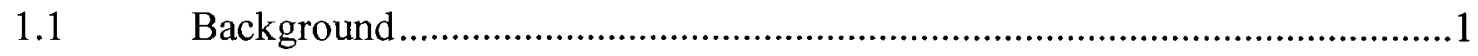

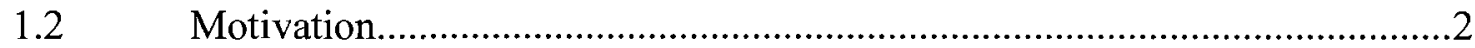

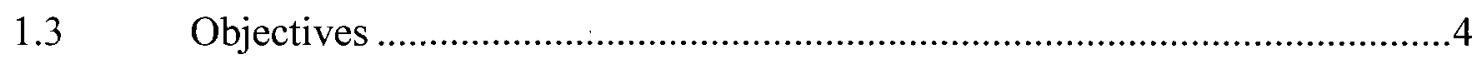

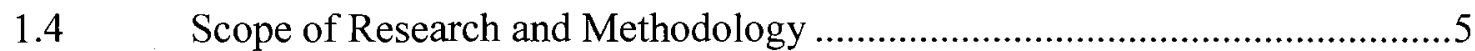

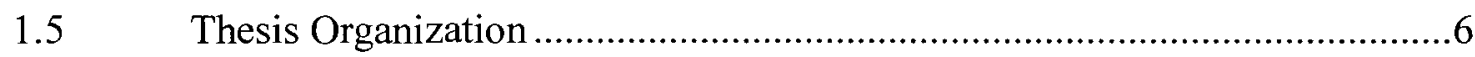

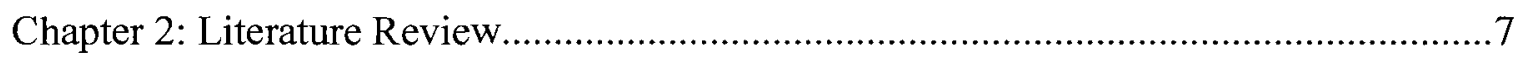

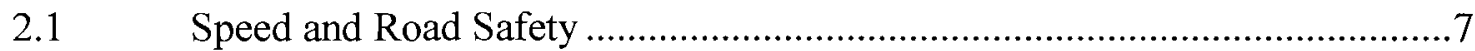

Speed and Its Influencing Factors.................................................. 12

2.2.1 Definition of Speed and Speeding .......................................................... 12

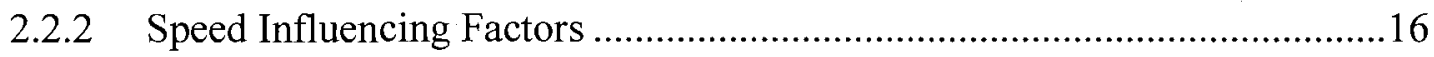

2.2.2.a Speed Influencing Factors Detected by Experiments ..................17

2.2.2.b Speed Influencing Factors Reported by Motorists .....................23

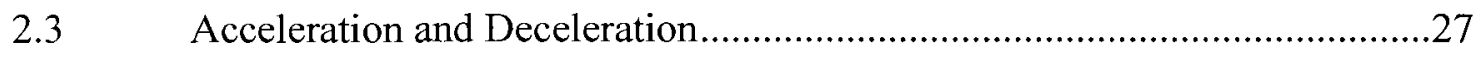

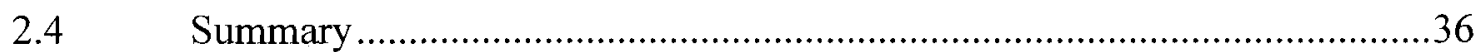

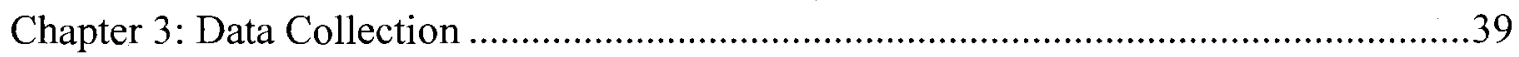

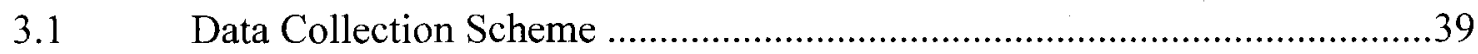




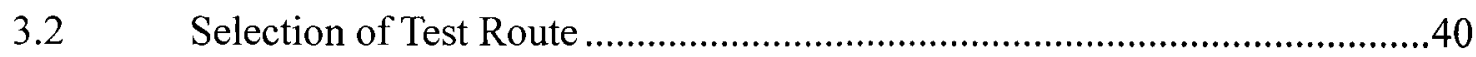

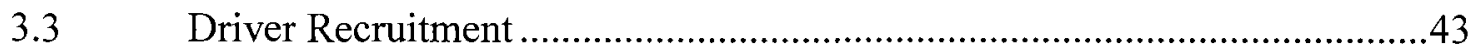

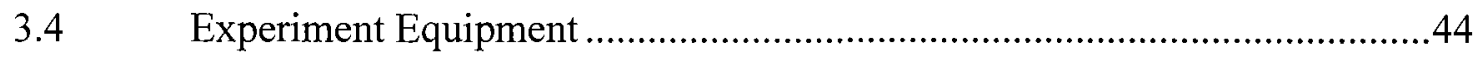

3.4.1 Carleton Data Collection System.................................................................

3.4.1.a Corsa Data Acquisition Box.............................................................46

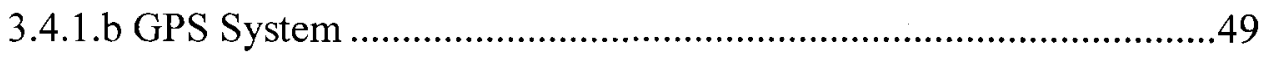

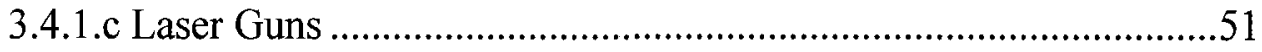

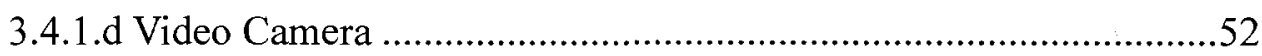

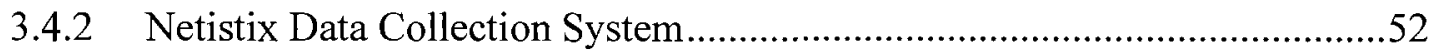

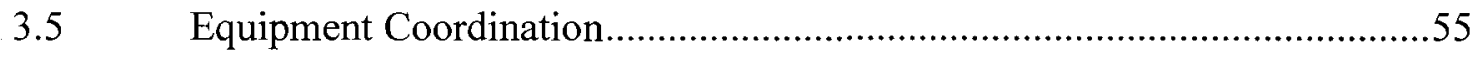

Chapter 4: Database Construction and Comparison of Two Measures ................................56

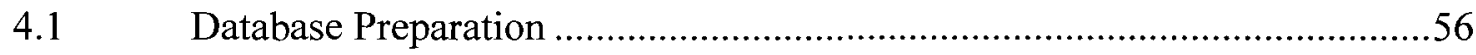

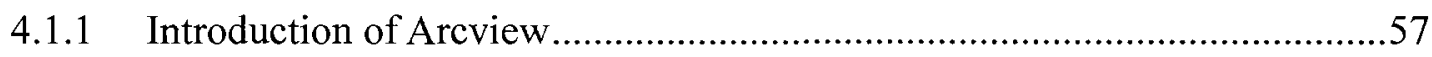

4.1.2 Procedure of Database Construction ..........................................................57

4.2 Speed Comparison ...........................................................................61

4.2.1 Speed Data Reduction...............................................................................61

4.2.2 Difference between VIU and Corsa Speeds .................................................62

4.2.3 Correlation Analysis of Speed Data................................................................65

4.2.4 Summary of Speed Comparison ..............................................................68

4.3 Throttle Data Comparison .........................................................................69

4.3.1 Difference of Raw Throttle data ..................................................................

4.3.2 Difference of Calibrated Throttle data ………...............................................73

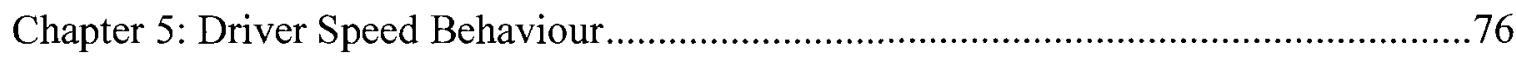




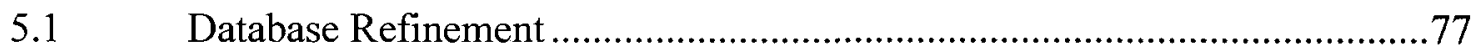

5.2 Driver Speed Behaviour on Different Road Classes .......................................81

5.2.1 Urban Freeway (Highway 417) ..............................................................

5.2.2 Two-Lane Rural Highways .........................................................................84

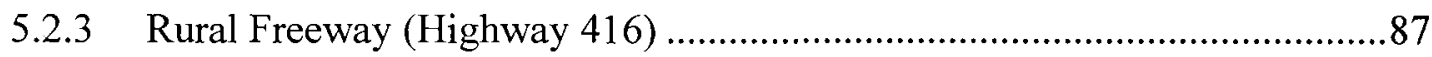

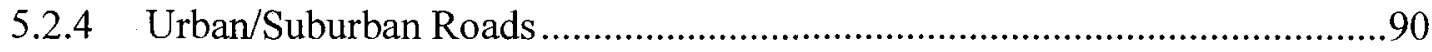

5.2.5 Summary of Driver Speed Behaviour on Different Road Classes..................93

5.3 Speed Behaviour of Different Driver Types .............................................98

5.3.1 Categorization of Driver Speed Behaviour...................................................98

5.3.2 Actual Driving Patterns versus Self-Reported Driving Patterns .................103

5.3.3 Speed Choice of the Three Driver Types.......................................................104

5.3.3.a Urban Freeway (Highway 417) …………………......................105

5.3.3.b Two-Lane Rural Highways .........................................................107

5.3.3.c Rural Highway (Highway 416)...................................................109

5.3.3.d Urban/Suburban Roads ..............................................................111

5.3.3.e Summary of Speed Choice of the Driver Types ...........................116

5.4 Framework for Judging Driving Aggressiveness .......................................117

Chapter 6: Driver Acceleration and Deceleration Behaviour ...........................................130

6.1 Computation of Instantaneous Acceleration Rates ....................................130

6.2 Driver Acceleration/Deceleration Behaviour on Different Road Classes ..132

6.2.1 Urban Freeway (Highway 417) ..............................................................133

6.2.2 Two-Lane Rural Highways .....................................................................137

6.2.3 Rural Highway (Highway 416) ...............................................................142 


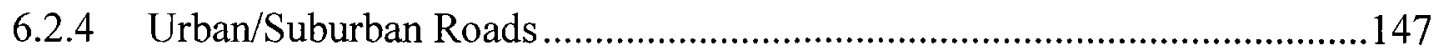

6.2.5 Comparison of Acceleration/Deceleration on Different Road Classes.......151

6.2.5.a Acceleration Behaviour...............................................................151

6.2.5.b Deceleration Behaviour .................................................................156

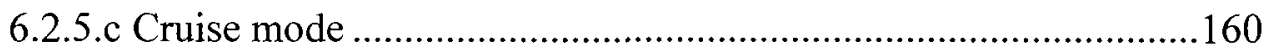

6.3 Acceleration/Deceleration Behaviour of Different Driver Types................162

6.3.1 Comparison of Acceleration Behaviour.....................................................163

6.3.2 Comparison of Deceleration Behaviour ....................................................164

Chapter 7: Conclusions and Recommendations ..........................................................166

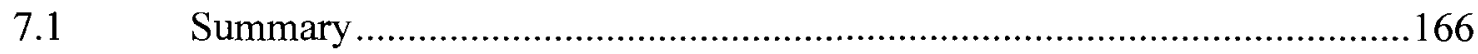

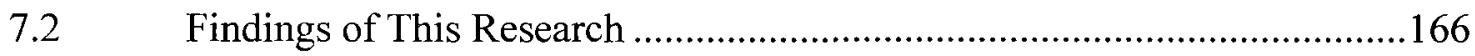

7.3 Contributions of This Research …………..........................................170

7.4 Recommendations for Future Study ....................................................170

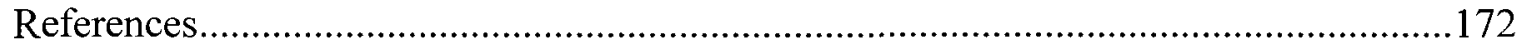

Appendix A: Speed Statistics of Individual Drivers for Different Road Classes .............181

Appendix B: Speed Distribution of Individual Drivers and the Estimated Speed

Distribution of the Common Driver Population ..............................................................194

Appendix C: Acceleration and Deceleration of Different Types of Drivers.....................221 


\section{LIST OF TABLES}

Table 2-1: Collision Location by Class of Collision (MTO, 2006; Table 3.13) ................8

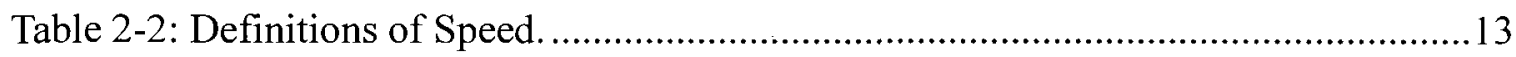

Table 2-3: Factors Affecting Drivers' Choice of Speed (ETSC, 1995). ..........................16

Table 2-4: Normal Acceleration and Deceleration Rates (ITE, 1982).........................28

Table 3-1: Seven Roads and Their Segment Lengths in the Test Route..........................41

Table 3-2: Summary of the Experimental Instruments and Data Types.......................45

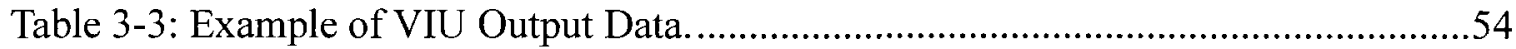

Table 4-1: The Speed Difference between the Two Systems (All Speed Pairs Included). 63

Table 4-2: The Speed Difference between the Two Systems (Excluded Speed Pairs if

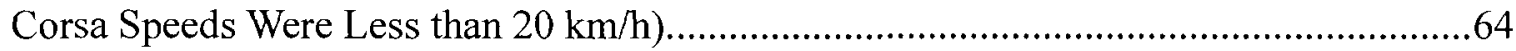

Table 4-3: Results of Correlation Analysis (All Speed Data Included).........................66

Table 4-4: Results of Correlation Analysis (Corsa Speeds Less than $20 \mathrm{~km} / \mathrm{h}$ )............66

Table 4-5: Results of Correlation Analysis (Corsa Speeds Equal to or Greater than 20

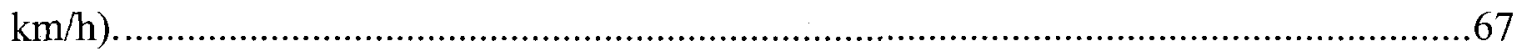

Table 4-6: The Difference of Throttle Data Using the Raw data................................72

Table 4-7: Minimum and Maximum Throttle Usages Recorded by the VIU and Corsa...73

Table 4-8: The Difference of Throttle Data Using the Calibrated data. ...........................74

Table 5-1: Speed Distribution for Urban Freeway (Highway 417). ............................83

Table 5-2: Speed Distribution for Two-Lane Rural Highways.....................................86

Table 5-3: Speed Distribution for Rural Freeway (Highway 416). .............................89

Table 5-4: Speed Distribution for Urban/Suburban Roads.................................... 92

Table 5-5: Summary of Statistics of Speeds on Different Road Classes. ........................94 
Table 5-6: Example of Distinguishing Driving Manners (Non-Free-Flow Conditions on Highway 416). 100

Table 5-7: Result of Judging Aggressive and Defensive Driving on the Four Road Classes. 102

Table 5-8: Comparison of Self-Reported and Actual Driving Patterns. 103

Table 5-9: Speed Distribution of Three Driver Types on Urban Freeway under Free-Flow Conditions 106

Table 5-10: Speed Distribution of Three Driver Types on Two-Lane Rural Highways under Free-Flow Conditions. 108

Table 5-11: Speed Distribution of Three Driver Types on Rural Freeway under Free-Flow Conditions. 110

Table 5-12: Speed Distribution of Three Driver Types on Urban/Suburban Roads under Free-Flow Conditions (PSL=50 km/h). 112

Table 5-13: Speed Distribution of Three Driver Types on Urban/Suburban Roads under Free-Flow Conditions $(\mathrm{PSL}=60 \mathrm{~km} / \mathrm{h}$ ) 113

Table 5-14: Speed Distribution of Three Driver Types on Urban/Suburban Roads under

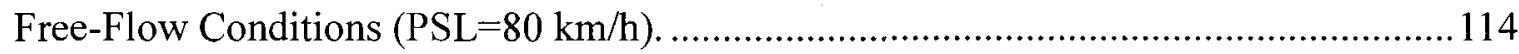

Table 5-15: Parameters for Judging the Aggressiveness of a Driver. ............................119

Table 5-16: Example of Observed and Predicted Speed Distributions...........................123

Table 5-17: Predicted 95\% Confidence Interval of Speed Distribution of the Common

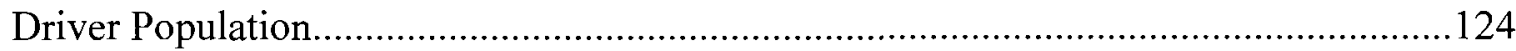

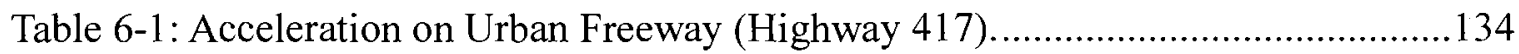

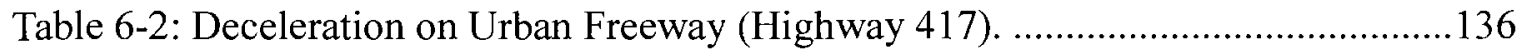


Table 6-3: Acceleration on Two-Lane Rural Highways.

Table 6-4: Deceleration on Two-Lane Rural Highways.

Table 6-5: Acceleration on Rural Freeway (Highway 416). 144

Table 6-6: Deceleration on Rural Freeway (Highway 416).

Table 6-7: Acceleration on Urban/Suburban Roads. 148

Table 6-8: Deceleration on Urban/Suburban Roads. 149

Table 6-9: Acceleration on Each Road Class under Free-Flow Conditions. 153

Table 6-10: Acceleration on Each Road Class under Non-Free-Flow Conditions. .154

Table 6-11: Deceleration on Each Road Class under Free-Flow Conditions. 157

Table 6-12: Deceleration on Each Road Class under Non-Free-Flow Conditions. 158

Table 6-13: Cruise Distribution on Each Road Class under Mixed-Flow Conditions.....161

Table 6-14: Statistics of Acceleration of the Three Driver Types under Mixed Flow Conditions. 164

Table 6-15: Statistics of Deceleration of the Three Driver Types under Mixed Flow Conditions 165 


\section{LIST OF FIGURES}

Figure 2-1: Four Common Acceleration Models (Wang et al., 2004)........................29

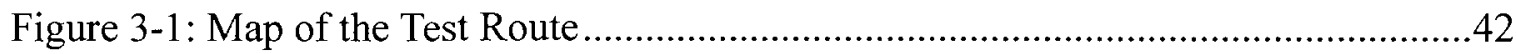

Figure 3-2: Test Vehicle and the Corsa Data Acquisition System. ...............................48

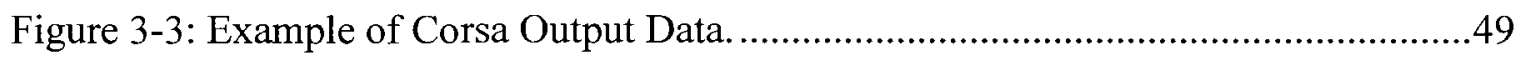

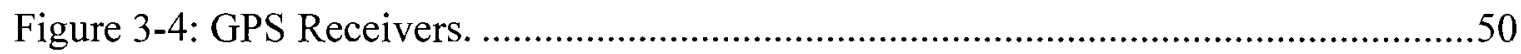

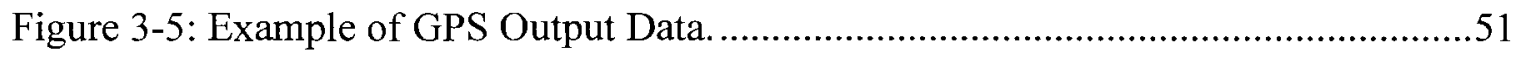

Figure 3-6: Laser Gun and Video Camera. ...............................................................52

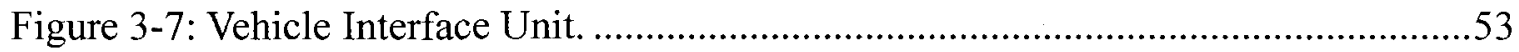

Figure 4-1: Procedure for Database Construction. ...................................................60

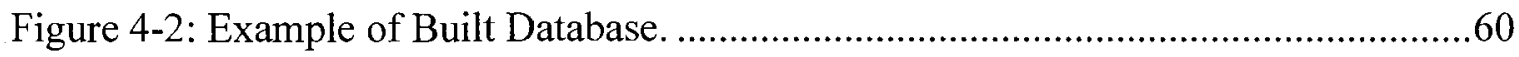

Figure 4-3: Example of Speed Difference. ...........................................................62

Figure 4-4: Example of Combined Throttle Data..................................................... 70

Figure 5-1: Example of Refined Database in Excel. ................................................77

Figure 5-2: Speed Distribution for Urban Freeway (Highway 417) ..........................84

Figure 5-3: Speed Distribution for Two-Lane Rural Highways. ..................................87

Figure 5-4: Speed Distribution for Rural Freeway (Highway 416) .............................90

Figure 5-5: Speed Distribution for Urban/Suburban Roads. ..................................93

Figure 5-6: Speed Choice in Relation to PSL on Different Road Classes under Free-Flow

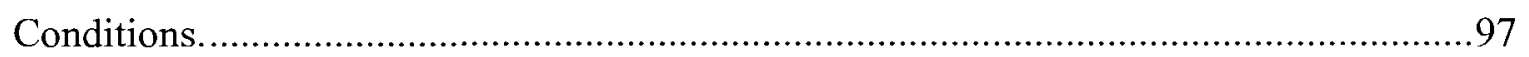

Figure 5-7: Speed Choice in Relation to PSL on Urban/Suburban Roads under Free-Flow

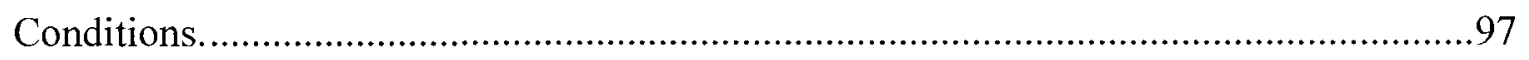

Figure 5-8: Procedure for Categorizing Driver Types. ............................................99 
Figure 5-9: Comparison of Self-Reported and Actual Driving Patterns. 104

Figure 5-10: Speed Choice of Three Driver Types on Urban Freeway under Free-Flow Conditions. 107

Figure 5-11: Speed Choice of Three Driver Types on Two-Lane Rural Highways under Free-Flow Conditions. 109

Figure 5-12: Speed Choice of Three Driver Types on Rural Freeway under Free-Flow Conditions 111

Figure 5-13: Speed Choice of Three Driver Types on Urban/Suburban Roads under FreeFlow Conditions (PSL $=50 \mathrm{~km} / \mathrm{h}$ ). 115

Figure 5-14: Speed Choice of Three Driver Types on Urban/Suburban Roads under FreeFlow Conditions (PSL $=60 \mathrm{~km} / \mathrm{h}$ ). 115

Figure 5-15: Speed Choice of Three Driver Types on Urban/Suburban Roads under FreeFlow Conditions $(\mathrm{PSL}=80 \mathrm{~km} / \mathrm{h}$ ). 116

Figure 5-16: Speed Distribution of Driver 02 and the Common Driver Population. ...... 125

Figure 5-17: Cumulative Frequency of Speed Distribution of Driver 02 and the Common

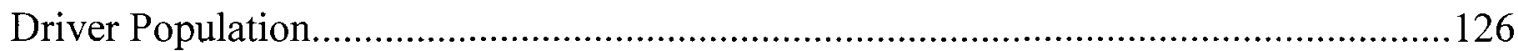

Figure 5-18: Speed Distribution of Driver 12 and the Common Driver Population. ...... 126 Figure 5-19: Cumulative Frequency of Speed Distribution of Driver 12 and the Common Driver Population. 127

Figure 5-20: Speed Distribution of Driver 17 and the Common Driver Population. ......127 Figure 5-21: Cumulative Frequency of Speed Distribution of Driver 17 and the Common

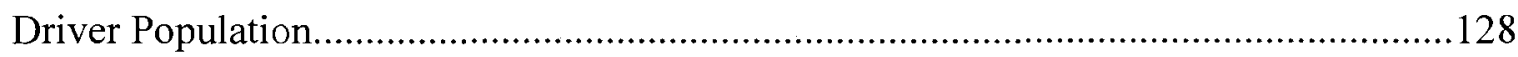

Figure 6-1: Example of Calculated Acceleration Rates in the Database. 132 
Figure 6-2: Acceleration on Urban Freeway (Highway 417)...........................................135

Figure 6-3: Deceleration on Urban Freeway (Highway 417) ……................................137

Figure 6-4: Acceleration on Two-Lane Rural Highways ................................................140

Figure 6-5: Deceleration on Two-Lane Rural Highways.................................................142

Figure 6-6: Acceleration on Rural Freeway (Highway 416).........................................146

Figure 6-7: Deceleration on Rural Freeway (Highway 416)...........................................146

Figure 6-8: Acceleration on Urban/Suburban Roads...................................................150

Figure 6-9: Deceleration on Urban/Suburban Roads....................................................150

Figure 6-10: Acceleration on Each Road Class under Free-Flow Conditions...................155

Figure 6-11: Acceleration on Each Road Class under Non-Free-Flow Conditions..........155

Figure 6-12: Deceleration on Each Road Class under Free-Flow Conditions..................159

Figure 6-13: Deceleration on Each Road Class under Non-Free-Flow Conditions. .......159

Figure 6-14: Cruise Distribution on Each Road Class under Mixed-Flow Conditions...162

Figure 6-15: Acceleration of the Three Driver Types under Mixed Flow Conditions.....164

Figure 6-16: Deceleration of the Three Driver Types under Mixed Flow Conditions. ...165 


\section{CHAPTER 1: INTRODUCTION}

\subsection{Background}

The highway system is fundamental to the development of the economies of communities, regions, and nations. In addition to directly transporting people and goods in an efficient manner, the highway system provides links among all modes of transportation. Canada and the USA are in the top list of vehicle ownership rates per capita in the world and highways are vital to the quality of life in North America. In the United States, more than 90 percent of passenger trips and 69 percent of total freight value are transported by the highway system (TRB, 2001). Canada has more than 900,000 kilometres of roads, which serve almost 19 million vehicles and over 21 million drivers nationwide (Transport Canada, 2004). In Ontario, there are over 16,500 kilometres of highways, on which about $\$ 1.2$ trillion worth of goods were transported in $2004(\mathrm{MTO}, 2006)$.

Despite the benefits resulting from the development of the highway system, road safety is gaining increased attention due to the fact that traffic collisions have become a major source of social pains and economic losses. In 2005, there were 43,443 people killed in motor vehicle traffic crashes, 2,699,000 people injured, and 4,304,000 crashes involving property damage only in the United States. This death toll means that on average, 119 persons died each day in motor vehicle crashes in the United States in 2005; i.e., one every 12 minutes (NHSTA, 2006a). In that same year, a total of 210,629 victims suffered from traffic collisions in Canada, of which 2,923 people were killed and 17,529 people were seriously injured (Transport Canada, 2007b). Over the past few years, the 
annual average fatalities on Canadian roads have hovered around 3,000 deaths, thus accounting for 95 percent of the transportation fatalities nationwide (Transport Canada, 2006). With consideration of health care costs, property losses, and other factors, the economic cost of traffic collisions to Canadians was as high as \$25 billion annually (Transport Canada, 2004). According to the Ontario Road Safety Annual Report (MTO, 2006 ), there were 231,548 total reportable collisions in Ontario in 2004 , involving 411,271 drivers and 426,951 vehicles. These collisions resulted in 799 fatalities, 3,565 serious injuries, and 29,918 minor injuries.

\subsection{Motivation}

With increased attention and awareness of road safety problems, numerous efforts have been made in the research and practices of transportation as well as the motor vehicle industry to reduce crash severity and collision involvement. The efforts in the discipline of transportation include improving roadway design consistency, providing more forgiving roadsides, developing safety devices, and enforcing traffic control. In addition, the motor vehicle manufacturing industry is improving vehicle crash avoidance capabilities and occupant protection through the voluntary enhancement of existing technologies and the introduction of innovative new technologies. Notable advancements include airbag systems, anti-lock brake systems, and adaptive cruise control systems.

In a driver-vehicle-road system, the relationships among the three components are complex and the driver is the most flexible but unstable component. Driver behaviour links directly with vehicle speed and safety. Although the improvements of highway design techniques and enhancements of vehicle qualities in the past decades have made 
impressive progress in transportation safety, positive changes in driver behaviour should be made as well since unsafe driving manners are a substantial contributing factor in a large portion of road accidents. Although numerous researchers have made tremendous efforts in solving the safety problems on roads through the study of driver behaviour, these research efforts and the associated findings have suffered a number of limitations.

The reliability and accuracy of outcomes in previous research could be questioned due to the absence of effective data collection in their studies. For example, many studies relied on using a radar gun for measuring vehicle speeds, which faces two problems. One is that drivers possibly change their speeds because of the presence of experimenters with the equipment. The other one is the cosine error resulting from the deviation between the radar gun beam and the vehicle travel direction, especially on curved roadways. In addition, the spot speeds were often detected by the devices fixed at or near the interested sites, examples of which are photography devices mounted at intersections or the speed detectors dispersed along a road section. These kinds of data collection methods make it impossible to track individual drivers for a long time. Unlike previous data collection methods, in this study a variety of devices were installed in an instrumented vehicle. These devices are capable of observing different measures providing abundant reliable information, which helps examine driver behaviour in detail.

In addition, most previous research focused on a single road class, especially two-lane rural highways. Admittedly, traffic crashes on undivided rural roadways account for a major part of the entire adversities on roads. However, it is very common now that people drive on different classes of roads in a single trip with the high numbers of vehicles in Canadian families and development of transportation networks. Traffic collisions on 
high-speed freeways often result in extremely serious consequences and those on urban streets could impose severe harm on vulnerable road users such as pedestrians and cyclists. Therefore, more efforts should address the issues related to other road types.

Few studies are available that investigate the difference of driver behaviour among the driver population. On one hand, it has been reported that different driver types may hold unique attitudes in their speed choice with relation to speed limits (Fitzpatrick et al., 2003). Many fatalities (12\%) and serious injuries (8\%) were found to be attributed to much fewer ( $3 \%$ to $4 \%$ ) high-risk drivers, who exhibit the most dangerous behaviours (Transport Canada, 2007a). The studies of driver aggressiveness put more emphasis on examining behaviours such as discourteous gestures and violations of traffic laws (Shinar, 1998; Raub et al., 2002). Little research has attempted a comprehensive analysis of driver speed behaviour, and the acceleration and deceleration behaviour for different driver types in one study.

\subsection{Objectives}

Based on the earlier explanation, it is imperative to perform a comprehensive study that explores driver performance on a diversity of road environments. Therefore, the main objective of this research is to investigate driver speed behaviour and acceleration/deceleration behaviour on different road classes in Canada. The findings of this research are expected to serve the following purposes:

- Recognizing driver speed behaviour on the most common road classes in Canada.

- Understanding the differences of driver acceleration and deceleration performance on different road classes. 
- Identifying different driver types based on their speed choice in the field experiment, and thus acknowledging the differences in acceleration (and deceleration) behaviour among these driver types.

It is hoped that the results in this research will serve as a reference for both transportation professionals and individual drivers to better understand driver behaviour on our roads. As a result, any improvement in both the practices of transportation engineering and public driving patterns would be expected to increase road safety in Canada.

\subsection{Scope of Research and Methodology}

To fulfill these objectives, a field experiment was conducted in real driving circumstances, which involved participation of 30 volunteer drivers. The recruited volunteer drivers were limited to those who were fully-licensed, as a driver sample for the majority of driver population in Ontario. A test route was intentionally pre-designated to cover the most common road classes for the experiment. The four road classes covered by the test route were an urban freeway, two-lane rural highways, a rural freeway, and urban/suburban streets. For practical reasons, the test route was selected in the vicinity of the City of Ottawa. The test runs were conducted during the daytime and under normal weather conditions in the fall of 2005. The experiment was limited to passenger cars only, using a Ford passenger minivan as the test vehicle. The vehicle was equipped with a variety of experimental devices.

During the experiment, the volunteer drivers drove the test vehicle on the test route according to their normal driving patterns. The devices installed in the test vehicle were 
capable of recording a variety of driver's operation data such as the instantaneous speeds, the use of fuel pedal, braking, and steering, etc. Employing GPS techniques, the vehicle's actual trajectories were tracked on the basis of coordinates provided by a GPS receiver on the test vehicle. Using the collected data, driver's speed behaviour, and acceleration and deceleration behaviour were analyzed afterwards.

\subsection{Thesis Organization}

This thesis comprises seven chapters. The first chapter presents research background, the study interests and objectives, the scope of the study, the framework of experiment, and the thesis structure. The second chapter gives a comprehensive review of previous literature pertaining to this study. Chapter 3 presents the details of data collection in this study, including introducing the experimental devices and describing data collected by these devices. Chapter 4 discusses the procedure of database construction and comparison of speed and throttle data collected by two sets of devices. Chapter 5 presents analysis of speed data on different road classes, identification of different driver types, and comparison of speed choice of these driver types. Chapter 6 provides analysis of acceleration and deceleration behaviours of the driver sample on the four road classes and comparison of acceleration and deceleration behaviours between the different driver types. The last chapter gives a summary of this research. 


\section{CHAPTER 2: LITERATURE REVIEW}

This chapter presents an extensive review of existing literature pertaining to driver speed and acceleration/deceleration behaviour, as well as the factors related to a driver's speed choice and a discussion of the relationship between speed and road safety. The relationship between speed and road safety is first examined on the basis of the latest statistical information in North America and the findings in previous research. Then the definitions of different speeds are stated according to the most recent design guides in North America. More emphasis is directed at reviewing literature relevant to studying factors that influence a driver's speed choice through either quantitative experiments or self-reports by motorists. Another important portion studies previous research pertinent to driver acceleration and deceleration behaviours. Summary and critical comments are presented at the end of this chapter.

\subsection{Speed and Road Safety}

Speed is one of the most prevalent factors contributing to traffic collisions. For example, 13,113 lives were lost in the USA in speeding related crashes in 2005, and speeding was a factor in 30 percent of all fatal crashes in that year. In addition, the economic cost of speeding-related crashes was estimated to be $\$ 40.4$ billion each year (NHSTA, 2006b). Due to the serious social pain and economic losses resulting from traffic crashes, transportation researchers and professionals keep on investigating the relationship between traffic crashes and driving speed.

According to the Ontario Ministry of Transportation (MTO) study for 2004 (MTO, 2006), in the 411,271 collisions on Ontario's highways, the majority of involved drivers 
$(321,486)$ were driving under normal conditions without impairment due to alcohol, drugs, or health problems. Moreover, as depicted in Table 2.1, almost half of the total collisions took place on roadway sections far from the influence of complicated roadway configurations like intersections, driveways, railway crossings, tunnels, and bridges. In addition, 62.5 percent of all collisions happened on dry road surfaces without the influence of bad weather. All the above information implies that a large number of collisions were not necessarily related to adverse factors such as complicated situations (e.g., intersections and railway crossings), bad weather, or driver impairment resulting from alcohol, drugs, or fatigue. Rather, the driving manner itself might contribute to the occurrence of traffic crashes.

Table 2-1: Collision Location by Class of Collision (MTO, 2006; Table 3.13).

\begin{tabular}{lrrrrrrrr}
\hline & \multicolumn{9}{c}{ Class of Collision } & & Total & $\%$ \\
\hline $\begin{array}{l}\text { Road } \\
\text { Location }\end{array}$ & Fatal & $\%$ & $\begin{array}{c}\text { Personal } \\
\text { Injury }\end{array}$ & $\%$ & $\begin{array}{r}\text { Property } \\
\text { Damage }\end{array}$ & $\%$ & & \\
\hline $\begin{array}{l}\text { Non- } \\
\text { intersection }\end{array}$ & 452 & 63.0 & 19,046 & 38.1 & 81,319 & 45.0 & 100,817 & 43.5 \\
\hline $\begin{array}{l}\text { Intersection } \\
\text { Related }\end{array}$ & 66 & 9.2 & 12,434 & 24.9 & 42,854 & 23.7 & 55,354 & 23.9 \\
\hline $\begin{array}{l}\text { At } \\
\text { Intersection }\end{array}$ & 118 & 16.4 & 12,939 & 25.9 & 31,897 & 17.6 & 44,954 & 19.4 \\
\hline $\begin{array}{l}\text { At/Near } \\
\text { Private Drive }\end{array}$ & 62 & 8.6 & 5,123 & 10.3 & 22,962 & 12.7 & 28,147 & 12.2 \\
\hline At Railway & 9 & 1.3 & 81 & 0.2 & 351 & 0.2 & 441 & 0.2 \\
\hline $\begin{array}{l}\text { Underpass or } \\
\text { Tunnel }\end{array}$ & 2 & 0.3 & 50 & 0.1 & 180 & 0.1 & 232 & 0.1 \\
\hline $\begin{array}{l}\text { Overpass or } \\
\text { Bridge }\end{array}$ & 8 & 1.1 & 219 & 0.4 & 987 & 0.5 & 1214 & 0.5 \\
\hline Other & 1 & 0.1 & 56 & 0.1 & 332 & 0.2 & 389 & 0.2 \\
\hline Total & 718 & 100 & 49,948 & 100 & 180,882 & 100 & 231,548 & 100 \\
\hline
\end{tabular}

There are two concerns regarding speed and road safety: the relationship between speed and collision severity, and the relationship between speed and collision 
involvement. With consideration of driver capability and vehicle physics, the Insurance Institute of Highway Safety (IIHS, 2007) listed the reasons why higher speeds would result in more collisions and more serious outcomes:

- A vehicle at a higher speed travels a longer distance during a fixed perceptionreaction time.

- A vehicle at a higher speed needs a longer distance to stop in an emergency, and thus increases the risk of collision.

- A vehicle at a higher speed is more prone to loss of control in an emergency, especially under adverse weather conditions.

- A vehicle at a higher speed results in more serious outcomes because the energy released during a collision is at a square power rate with a vehicle speed.

It is widely accepted that higher driving speeds lead to a dramatic increase in collision severity according to the laws of physics and as indicated in some studies. For example, Pasanen and Salmivaara (1993) studied the relationship of free vehicle speeds and safety of pedestrians using the real-life accident information recorded by video-tape in Helsinki, Finland. They claimed that pedestrian safety depends to an alarming degree on vehicular speed. For instance, a speed of $50 \mathrm{~km} / \mathrm{h}$ increases the risk of pedestrian death almost eight-fold compared to a speed of $30 \mathrm{~km} / \mathrm{h}$.

However, the relationship between speed and collision involvement is complicated, and researchers hold different views to this issue based on their own research. For example, Kloeden et al. (1997) stated that the relative risk of being involved in a casualty crash approximately doubles for each $5 \mathrm{~km} / \mathrm{h}$ increase in free traveling speed above the 
speed limit of $60 \mathrm{~km} / \mathrm{h}$. On the other hand, Rodriguez (1990) found that the average speed of the driving population has a negative correlation with the fatality rate, and Lave (1985) did not find a significant relationship between the average speed and fatality rate.

Using speed and accident data on rural highways in the United States in the 1950s, Solomon (1964) found that if a vehicle travels either too slow or too fast related to the average speed, the vehicle is more likely to be involved in a crash. Garber and Gadiraju (1989) also indicated that speed variance is an important factor affecting road safety, and that accident rates do not necessarily increase with an increase in average speed. However, accidents do increase with an increase in speed variance. Moreover, other researchers (e.g., Lave, 1985; Fowles and Loeb, 1989; Levy and Asch, 1989; Snyder, 1989; Rodriguez, 1990) also affirmed that speed variance positively affects the fatality rate. Garber and Gadiraju (1989) indicated that speed variance is mainly due to the inconsistency between the design speed and the posted speed limit since drivers tend to drive faster with the improvement of roadway geometric characteristics, regardless of the posted speed limit.

Finch et al. (1994) studied the relationship between changes in mean speed and concurrent changes in numbers of accidents. This study was based on rich speed and accident data on various types of roads in Denmark, Finland, Germany, Sweden, Switzerland, the UK, and the USA. The authors concluded that a $1 \mathrm{~km} / \mathrm{h}$ increase in mean traffic speed typically results in a 3 percent increase in accident frequency.

A similar trend was also reported by Liu and Popoff (1997), who examined the relationship between travel speed and collision involvement on Saskatchewan provincial highways using speed data and the corresponding accident data for 26 years from 1969 to 
1994 in Saskatchewan, Canada. They found that casualties would be reduced by about 7 percent for every $1 \mathrm{~km} / \mathrm{h}$ reduction in average travel speed and casualty rates on provincial highways were positively correlated to speed differentials. The authors also reported that speed-related human errors contributed to 30 percent of all collisions and more than 40 percent of all casualty collisions in Saskatchewan. These speed-related errors included exceeding speed limits, driving too fast for road conditions, following too closely, failing to yield, and disregarding traffic control devices.

Feng (2001) reviewed the previous literature pertaining to speed and road safety and discussed the factors that affect speed and safety. Factors including environment, distraction, headway, and speed limit were mainly explained in relation to safety. The influence of speed limit, environment, driver behaviour, and advisory and regulatory information was discussed in relation to speed. The author stated that the factors of environmental conditions, driver behaviour, and speed limits were strongly related to both speed and safety. The author suggested that a speed limit must reflect real-time road, traffic, and weather conditions; otherwise it would be easily violated by drivers. In addition, it was suggested that subjective methods should not be heavily relied upon as drivers may not always accurately rate their driver behaviour. Moreover, environmental factors, such as rain, ice, etc., were found to have a close relationship to speed and safety since they had an impact on driver visibility, and vehicle stability and controllability.

Jun et al. (2007) assessed the differences of driving behaviour activity patterns in terms of mileage exposures, speed patterns, and acceleration patterns between two driver groups of crash-involved drivers and non-crash-involved drivers for a 14-month study period. The second-by-second vehicle activities, including vehicle position and vehicle 
speed, were observed by a GPS unit instrumented in participants' vehicles. Also considered was the driver had been involved in a previous crash as self-reported by the driver. Based on the comparison of activity patterns, crash-involved drivers usually traveled longer mileage, normally drove at higher speeds than non-crash-involved drivers, and employed hard deceleration more frequently.

\subsection{Speed and Its Influencing Factors}

\subsubsection{Definition of Speed and Speeding}

Speed is a fundamental factor in transportation engineering; it is often denoted by different terms while applied in different situations such as a design criterion, a measure of the level of service and an operational control parameter. Table 2-2 contains speed definitions in recent publications of the North American guides: A Policy on Geometric Design of Highways and Streets (AASHTO, 2004), Manual of Uniform Traffic Control Devices (MUTCD, 2003), and Geometric Design Guide for Canadian Roads (TAC, 1999).

The design speed concept was initially used in 1930s in highway geometric design in Germany and the United States. In the AASHTO design guides of 1994 and earlier, the design speed was denoted as the "maximum safe speed" that can be maintained over a section of highway under favourable conditions. However, the "safe" term was removed in the Green Books of AASHTO after 1994 due to the recognition that speeds higher than the design speed do not necessarily result in safety problems, and that speeds lower than the design speed are not crash-free. 
Table 2-2: Definitions of Speed.

\begin{tabular}{|c|c|c|}
\hline Measure & Reference & Definition \\
\hline \multirow{3}{*}{$\begin{array}{l}\text { Design } \\
\text { Speed }\end{array}$} & $\begin{array}{l}\text { MUTCD } \\
2003\end{array}$ & $\begin{array}{l}\text { a selected speed used to determine the various } \\
\text { geometric design features of a roadway }\end{array}$ \\
\hline & $\begin{array}{l}\text { AASHTO } \\
2004\end{array}$ & $\begin{array}{l}\text { a selected speed used to determine the various } \\
\text { geometric design features of the roadway }\end{array}$ \\
\hline & $\begin{array}{l}\text { TAC } \\
1999\end{array}$ & $\begin{array}{l}\text { a speed selected as a basis to establish appropriate } \\
\text { geometric design elements for a particular section } \\
\text { of road. }\end{array}$ \\
\hline \multirow{3}{*}{$\begin{array}{l}\text { Operating } \\
\text { Speed }\end{array}$} & $\begin{array}{l}\text { MUTCD } \\
2003\end{array}$ & $\begin{array}{l}\text { a speed at which a typical vehicle or the overall } \\
\text { traffic operates. Operating speed may be defined } \\
\text { with speed values such as the average, pace, or } \\
\text { 85th percentile speeds. }\end{array}$ \\
\hline & $\begin{array}{l}\text { AASHTO } \\
2004\end{array}$ & $\begin{array}{l}\text { the speed at which drivers are observed operating } \\
\text { their vehicles during free-flow conditions. The } \\
85 \text { th percentile of the distribution of observed } \\
\text { speeds is the most frequently used measure of the } \\
\text { operating speed associated with a particular } \\
\text { location or geometric feature. }\end{array}$ \\
\hline & $\begin{array}{l}\text { TAC } \\
1999\end{array}$ & $\begin{array}{l}\text { the speed at which a driver is observed operating a } \\
\text { vehicle (a "spot" speed at a particular location). } \\
\text { The operating speed of all vehicles at a particular } \\
\text { location is reported as either a mean or } 85 \text { th } \\
\text { percentile operating speed. }\end{array}$ \\
\hline $\begin{array}{l}\text { Average } \\
\text { Speed }\end{array}$ & $\begin{array}{l}\text { MUTCD } \\
2003\end{array}$ & $\begin{array}{l}\text { the summation of the instantaneous or spot- } \\
\text { measured speeds at a specific location of vehicles } \\
\text { divided by the number of vehicles observed. }\end{array}$ \\
\hline \multirow{3}{*}{$\begin{array}{l}\text { Posted } \\
\text { Speed }\end{array}$} & \begin{tabular}{|l} 
MUTCD \\
2003
\end{tabular} & $\begin{array}{l}\text { the speed limit determined by law and shown on } \\
\text { Speed Limit signs. }\end{array}$ \\
\hline & $\begin{array}{l}\text { AASHTO } \\
2004\end{array}$ & $\begin{array}{l}\text { Posted speed limits, as a matter of policy, are not } \\
\text { the highest speeds that might be used by drivers. } \\
\text { Instead, such limits are usually set to approximate } \\
\text { the } 85 \text { th-percentile speed of traffic as determined } \\
\text { by measuring the speeds of a sizable sample of } \\
\text { vehicles. }\end{array}$ \\
\hline & $\begin{array}{l}\text { TAC } \\
1999\end{array}$ & $\begin{array}{l}\text { a speed limitation, consciously introduced for } \\
\text { reasons of safety and economy, traffic control and } \\
\text { government regulatory policies. }\end{array}$ \\
\hline
\end{tabular}


The Green Book (AASHTO, 2004) presents the determining factors in selecting a design speed. These factors include the topography, anticipated operating speed, the adjacent land use, and the functional classification of highways. Moreover, the Green Book depicts other considerations with respect to the choice of design speed: design speed should be consistent with drivers' expected speed; design speed should fit expected speed for nearly all drivers; and design speed should consider the average trip length. The definition of design speed and the determining factors in selecting a design speed in the TAC (1999) guide are identical to that in the Green Book. However the Green Book provides specific guidance in more detail for the selection of design speed than the TAC guide.

After the design speed is selected, diverse roadway parameters such as horizontal curve radius, superelevation, and vertical curve length should be appropriately determined in accordance to the value of design speed. The recent publications of AASHTO Green Books (2001 and 2004) and the TAC (1999) guide avoid the term of "standard", and the choice of the above parameters in highway facility design relies mainly on the judgement and experience of transportation practitioners. However, the design speed method has some limitations in determining highway facility parameters, as pointed out by Krammes (2000). A fundamental limitation is that the design speed applies directly to horizontal curves and not to tangents between these curves. Another limitation is that the selected parameters, even though they are all above the minimum values, cannot ensure consistency between successive elements (e.g., horizontal curves) of the highway alignment. 
With the development of current design procedures, posted speed limits are set based on statistical analysis of the individual vehicular speeds observed along the roadways. Based on the review of existing methods regarding how to set the speed limits, Fitzpatrick et al. (2003) found that the $85^{\text {th }}$ percentile speed is the predominant factor in setting speed limits in the states of the USA. Furthermore, it was found that roadway geometry, accident experience, and roadside development are the most popular factors considered by most agencies when they set speed limits.

Speeding is another concept that often appears in literature. "The term 'speeding' is used to describe the behaviour of a driver who is operating a vehicle at a speed which is considered too fast for the prevailing conditions or at a speed greater than that specified by the posted speed limits" (Zaal, 1994). MTO (2006) and the National Highway Traffic Safety Administration (NHTSA, 2006b) gave the same description for speeding. The European Transport Safety Council (ETSC, 1995) used "excess speed" to denote speed exceeding the speed limit and "inappropriate speed" to denote the speed that is too fast for the prevailing conditions. In fact, all the above agencies give an almost identical description of speeding.

Speeding is one of the most prevalent factors contributing to traffic accidents. As noted in the report of MTO (2006), drivers who exceed the speed limit by $30 \mathrm{~km} / \mathrm{h}$ or more have 6 times the probability of injuring or killing themselves or other road users as compared to those who obey the speed limits. ETSC (1995) indicated that more than 11,000 deaths and 180,000 injuries caused by accidents could be avoided annually in the European Union if the average speed is reduced by just $5 \mathrm{~km} / \mathrm{h}$. 


\subsubsection{Speed Influencing Factors}

In order to understand better the mechanism of how speed works, it is necessary to examine carefully what stands behind speed. The Institute of Transportation Engineers (ITE, 1992), from an aggregate perspective, presented and described five travel factors that influence traffic speeds: driver factors, vehicle factors, roadway factors, traffic factors, and environmental factors. How an individual driver chooses to drive a particular vehicle is the outcome of a complex process, which involves personal characteristics and circumstances as well as the type of road, its layout and surroundings, the amount and composition of the traffic, and the prevailing environmental conditions (ETSC, 1995). The factors that influence the driver's behaviour in speed choice can be divided into three categories: road and vehicle related, traffic and environment related, and driver related factors. Table 2-3 gives a list of these factors

Table 2-3: Factors Affecting Drivers' Choice of Speed (ETSC, 1995).

\begin{tabular}{|l|l|l|}
\hline $\begin{array}{l}\text { Road and } \\
\text { Vehicle related }\end{array}$ & $\begin{array}{l}\text { Traffic and } \\
\text { Environment related }\end{array}$ & Driver Related \\
\hline Road: & Traffic: & age \\
width & density & gender \\
gradient & composition & reaction time \\
alignment & prevailing speed & attitudes \\
surroundings & Environment: & thrill seeking \\
layout & weather & risk acceptance \\
markings & surface condition & hazard perception \\
surface quality & natural light & alcohol level \\
Vehicle: & road lighting & ownership of vehicle \\
type & signs & circumstances of journey \\
power/weight ratio & speed limit & occupancy of vehicle \\
maximum speed & enforcement & \\
comfort & & \\
\hline
\end{tabular}




\subsection{2.a Speed Influencing Factors Detected by Experiments}

Driving involves an interaction between the driver, vehicle, and roadway environment. While driving, the driver perceives the immediate scenario he/she encounters and thus takes a favourable manoeuvre accordingly. Two factors are generally deemed significant in affecting driver behaviour (Chakroborty et al. 2004); one is the driver's concern for safety and the other is the driver's urge to reach his/her destination as soon as possible. The two factors, together with the existing traffic rules and regulations, determine the perception-reaction mechanism of every driver in all driving situations. Extensive research of driver behaviour has been performed in relation to these factors, intending to improve the performance of highways in terms of safety and efficiency.

Van Aerde and Yagar (1983) reported the effects of traffic volume on speeds of twolane rural highways, using the speed and volume data collected on Ontario highways in 1980. They derived regression equations for $10^{\text {th }}, 50^{\text {th }}$, and $90^{\text {th }}$ percentile free-flow speeds predicted by variables of traffic volumes. The variables represented volumes of different vehicle types in the mainline direction and total traffic volume in the opposite direction. The authors observed that these three percentile speeds were generally quite insensitive to traffic volumes under the normal operating condition (i.e., without flow breakdown), while they tended to converge as volume increased to flow breakdown and queuing. In addition, the authors found that traffic volumes presented more impact on the faster vehicles, represented by the $90^{\text {th }}$ percentile, than on the slower vehicles, represented by the $10^{\text {th }}$ percentile.

Yagar and Van Aerde (1983) studied the influence of diverse highway properties (e.g., curvature, grade, land use, lane width, etc.) within a 1500-m stretch upstream on the spot 
speeds of interested sites. They found that, on two-lane rural highways in Ontario, adjacent land use and legal speed limit imposed the most significant impact on speeds, followed by elements of grade, access, and lane width. However, the factors of road curvature, extra lane, sight distance, centerline markings, and lateral obstructions were not found to have a statistically significant effect on the variation of speeds. The findings in their study were not in accordance with that of many others. The authors explained that the relatively high and uniform design standard of Ontario highways may contribute to the findings. However, the treatment of exponentially averaging the geometric properties within 1500-m upstream may have produced a bias since the influence of some variables could be overestimated or underestimated using their average values.

Kanellaidis et al. (1990) investigated the influence of various geometric design parameters on driver speed behaviour on horizontal curves on rural roads. The geometric parameters included the radius of curvature, desired speed, superelevation rate, lane width, shoulder width, and length of curve. The authors found that the operating speed was substantially related to the horizontal curvature and the driver's desired speed.

Fitzpatrick et al. (1997) investigated the relationship between design speed and operating speed on suburban arterials using speed data collected on horizontal and vertical curves on suburban arterials in Texas. In their research, vehicle speeds were measured using laser guns or radar guns. The speed data of the drivers that may have reacted to the data collection process were discarded and only free-flow speeds with a minimum headway of 5-second were applied in the study. The authors analyzed the effects of only two variables on operating speeds on horizontal or vertical curves since the two factors were deemed, by the authors, to have the most significant influence on 
driver speed behaviour and to affect other variables. The two variables were curve radius and access density for horizontal curves and inferred design speed and access density for vertical curves. Inferred design speed was a calculated design speed using current design policy and known variables like vertical-curve length and grades. The authors found that drivers operated at speeds greater than the inferred design speed of $70 \mathrm{~km} / \mathrm{h}$ or less on suburban horizontal curves. By contrast, drivers used greater speeds than the inferred design speeds of $90 \mathrm{~km} / \mathrm{h}$ or less on horizontal curves on two-lane rural highways.

Gattis and Watts (1999) presented their findings of the relationship among vehicle speed, street width, and street function (arterial versus local) for six two-lane urban streets in Fayetteville, Arkansas. Their findings suggested that street width might have a small influence on vehicle speed, but other factors such as street function might impose more significant effects on the average and $85^{\text {th }}$ percentile speeds. In fact, they tentatively inferred that observed speeds were related more to the expected travel distance before stopping rather than road type and street width.

Ericsson (2000) compared driving patterns in terms of speed and acceleration between different street configurations, traffic conditions, and types of drivers in an urban area. In the study, the test route contained four street types: main street in a residential area, local feeder road in a residential area, radial arterial towards the city center, and street in the city center. Twelve drivers conducted the test for both peak hours and offpeak hours. The author found that the average speed and average acceleration differed significantly for all street types, and that streets with high average speed tended to have low average acceleration and deceleration levels, and vice versa. Consequently, the 
author concluded that street type imposed the greatest influence on the driving pattern, followed by the driver factor of gender.

Ottesen and Krammes (2000) studied the operating speeds on 138 horizontal curves and 78 approach tangents for 29 two-lane rural highways in five states in the USA. Based on the statistical analysis, they found that degree of curvature, length of curvature, and deflection angle significantly influenced vehicle speeds on curves. No satisfactory model was found to predict the operating speed with road characteristics.

Fitzpatrick et al. (2001) investigated how the design parameters of the road alignment, cross section, roadside, and traffic control device affect driver behaviour on four-lane suburban arterials. Alignment factors in terms of curve radius, curve length, and deflection angle were included in the horizontal curve analysis, while straight section length was used for the straight section analysis. Cross section factors considered included lane width, superelevation rate, and median type and width. Roadside factors included roadside development, access density, roadside environment, and pedestrian activity. Traffic control factors consisted of signal spacing, posted speed limit, and presence of advisory signs. The relationship between the operating speed $\left(85^{\text {th }}\right.$ percentile free-flow speed) and the above-mentioned factors was investigated for both horizontal curves and straight sections. The authors found that posted speed limit was the most significant variable for both straight and curved sections if all variables were considered. On the other hand, if posted speed limit was not used in the analysis, lane width was a significant variable for the straight sections, while median presence and roadside development were significant for the curved section. 
Fitzpatrick et al. (2003) examined speeds under free-flow conditions at a number of sites on various functional roadway classes to investigate the relationship between speeds of different percentiles and the posted speed limit. They found that the $50^{\text {th }}$ percentile speed was closest to the posted speed with a coefficient of determination $R^{2}$ of 0.911 . Furthermore, the $85^{\text {th }}$ percentile speed was predicted to increase in a nearly linear manner with the increase of the posted speed limit, and this estimated speed was more than 7 $\mathrm{mi} / \mathrm{h}$ above the posted speed limit. The authors, in addition, found that there were perhaps three types of drivers in terms of their speed choice behaviours, based on the speed distribution plots. Conservative drivers always tried to keep speed below the posted speed limit; moderate drivers, who accounted for the majority of drivers, generally tried not to exceed speed limit to unreasonable extents; and aggressive drivers, who considered speed limits as the bottom bound, usually attempted to drive at higher speeds.

Giles (2003) studied spot speeds relevant to different road environments using speed data observed on 79 rural and 115 metropolitan roads in Western Austria. Different from other studies that included only passenger vehicles, this study considered different types of vehicles aggregately. Four seconds was used as the threshold to select free-flow speeds. The author found that two-thirds of drivers traveled at speeds within 10 to $14 \mathrm{~km} / \mathrm{h}$ of the speed limit and the speed deviation from the speed limit tended to be larger under higher speed limits. The author indicated that drivers chose vehicle speeds based on the factors of road environment and vehicle other than the desire to be non-compliant.

Chakroborty et al. (2004) proposed a comprehensive microscopic model for driver behaviour in uninterrupted traffic flow, which is used to predict the driver behaviour of both steering control and speed control in free-flow conditions or forced-flow conditions. 
The drivers analyzed driver behaviour in a variety of driving scenarios such as carfollowing, overtaking, and reacting to on-coming vehicles. The factors of road edge, lane marking, and static obstacle were considered in the research. However, this model is limited to a two-way traffic circumstance without consideration of near-capacity or stopand-go conditions.

Ogle (2005) presented a framework and methods for quantifying and analyzing individual driver behaviour using 172 instrumented vehicles from the Commute Atlanta Program. The speed and location of each vehicle were captured second by second using a GPS unit and other devices installed in the vehicle. She found that nearly 40 percent of all driving activities in the sample population were above the posted speed limit, and approximately 12 percent of the driving activities took place at more than $10 \mathrm{mi} / \mathrm{h}$ above the posted speed. Furthermore, the amount and extent of speeding were found to decrease with the increase of the driver's age.

Nesamani et al. (2006) investigated the extent of the influence of traffic characteristics, roadside characteristics, and geometric elements over the vehicle average speed on urban arterials in the heterogeneous conditions in India. The test vehicle speeds were recorded by GPS at one second intervals. The traffic characteristics considered included vehicle composition and traffic flow. The geometric characteristics considered comprised four factors: lane width, grade, road quality (pavement condition), and road type (straight or curved section). The roadside characteristics considered included pedestrian movements, parking activities, adjacent land uses, and cross traffic access. The authors found that the traffic factors had the most significant influence over the variation in speed, followed by roadside characteristics and geometric design factors. According to 
the research, only the variable of lane width in geometric factors was found to be significant for the speed variation, and the road type of tangent or curve was not significant.

EI-Basha et al. (2007) conducted a study regarding driver speed and deceleration behaviour on deceleration lanes preceding the exit ramp terminals on an urban freeway. Vehicle speeds were measured on Highway 417 in the city of Ottawa using laser guns. Driver speed behaviour was represented in parameters of $85^{\text {th }}$ percentile diverge speed and $85^{\text {th }}$ percentile speed at gore area in exiting operations. Driver deceleration behaviour was examined using parameters of $85^{\text {th }}$ percentile average deceleration rates and $85^{\text {th }}$ percentile maximum deceleration rates on speed change lanes. The authors produced multiple regression models in which these speed or deceleration parameters could be estimated using other predictors in terms of highway geometric parameters (e.g., length of deceleration lane, and divergence angle between exit ramp and the right through lane), and traffic characteristics (e.g., hourly traffic volume and mean speed of right through lane). The authors found that the factors of mean speed on freeway right lane, length of deceleration lane, divergence angle, and diverging traffic volume created most of the influence on the diverge speed.

\subsection{2.b Speed Influencing Factors Reported by Motorists}

The literature in the previous section addresses mainly the factors that may influence driving speed through experiments or other forms of data collection of some quantitative speed measures. This section addresses the factors that may determine driver speed choice, according to the motorists, as identified through questionnaires or telephone 
surveys. Results in this part can spell out how road users rank the importance of various factors in affecting their driving speed.

In the research by Kanellaidis (1995), a questionnaire was conducted to collect drivers' attitudes in rating 14 elements of the road environment that influence their speed choice on road curves. The fourteen elements were pavement condition, number of traffic lanes, lane width, existence of free roadside space, existence of median, existence of safety barriers, sharp curvature, standard curve-warning signing, additional warning signing, speed-limit signing, superelevation rate, sight distance, length of curve, and gradient. Four out of the 14 elements were rated to have the most influence on drivers' choice of speed on curves: separation of opposing traffic, cross-section characteristics, alignment, and signage. Although the research proposed elements that were only related with speed choice on interurban road curves, the results provided an insight into the significances of various roadway factors in affecting drivers' operating speeds, particularly while vehicle traversed curved road sections.

In 1997, NHTSA (1998) conducted a national survey of the driving public in the USA. A total of 6,000 effective surveys were completed by phone interviews. The basic speedrelated questions and the corresponding responses are shown below.

(1) Drivers were asked how important a series of factors was in selecting their speeds.

- The most important factor was the weather condition, where 86 percent of the respondents felt that weather was extremely important and another 10 percent felt that it was moderately important. 
- The second most important factor was the posted speed limit, where 54 percent of the respondents rated it as extremely important and an additional 35 percent rated it as moderately important.

- The third most important factor was past experience on that road. More than 84 percent of the interviewees rated it as extremely important $(48 \%)$ or moderately important $(37 \%)$.

- The next three important factors included traffic density, the chances of being stopped by police, and the speed of other traffic.

(2) Drivers were asked why they considered driving at speeds above the maximum speed to be unsafe.

- For rural non-interstate roads, the most important factors were road condition, traffic patterns and flow, the presence of people, safety, drivers' reaction time and the braking ability of the vehicle.

- For interstate highways, the most important factor was safety due to the likelihood of losing control. The second factor was the drivers' reaction time and the braking ability of the vehicle.

(3) The drivers who reported that they drove faster now than they did one year ago were asked the reasons. The three most important reasons reported by the drivers were increased speed limits ( $52 \%$ of respondents), the increased experience of the driver (18\% of respondents), and improved traffic flow.

(4) Similarly, those who reported that they now drove slower than one year ago were asked the reasons. Two in five drivers mentioned driver-related reasons, especially the maturity of the driver. More than one third reported safety 
concerns, of which about half of the concerns were related to driving more cautiously. About 13 percent of respondents reported they drove slower to avoid crashes or because they had been involved in a crash. Other reasons resulting in driving slower were related to vehicle or traffic enforcement factors.

(5) The drivers were asked the possible causes resulting in more aggressive driving if they reported that other drivers were driving more aggressively in their area. Around 23 percent of drivers thought that drivers drove more aggressively because they were in a hurry or behind schedule. Another 22 percent of the drivers thought that this was due to increased traffic volume and congestion.

NHTSA (2002) undertook another survey in 2002, which was similar to the survey in 1997. A total of 4,010 drivers throughout the USA were involved in the latter survey. One question was how important various factors were in determining drivers' speed choice on different types of roads. The four road types covered in the survey were multi-lane interstate highways; non-interstate multi-lane roads; two-lane roads with posted speed limits of $45 \mathrm{mi} / \mathrm{h}$; and city, town, or neighbourhood streets. Across different road types, the top five most important factors were weather conditions, driver's personal assessment of safe speed, posted speed limits, traffic volume, and driver's personal experience on that road. Comparing the two surveys, it is found that most results of the latter survey appear to be unchanged from 1997. On the other hand, drivers reported more aggressive driving in their area than in the 1997 survey. 


\subsection{Acceleration and Deceleration}

Acceleration and deceleration rates are a critical factor in determining highway design features such as intersection, ramps, climbing lanes, passing zone, and stopping distance (AASHTO, 2004). Two kinds of acceleration (or deceleration) rates often appear in publications: maximum rate and normal rate. The maximum acceleration rate of different vehicles depends on the vehicle's size, weight, and engine power. Vehicle maximum acceleration capabilities are a factor for evaluation of passing zone lengths on two-lane highways and determination of minimum lengths of acceleration lanes at interchanges (ITE, 1992). The maximum deceleration rates are determined by the effective coefficient of friction at the tire-pavement surface. This coefficient of friction depends on the pavement type, tire condition, and whether the pavement surface is dry or wet. Maximum deceleration rates are used for estimating minimum stopping sight distance in emergencies. However, maximum acceleration and deceleration rates are seldom used in normal driving, except in emergency situations.

In normal driving, drivers usually apply reasonably comfortable acceleration and deceleration rates. Therefore, normal acceleration and deceleration rates are a factor that is often applied in diverse practices. For example, normal acceleration rates are used for determining cycle lengths of traffic signals, and computing fuel and travel time values (ITE, 1992). Normal deceleration rates are a basis for estimating reasonable time and road lengths for stops at signs and signals (ITE, 1992). The typical values of acceleration and deceleration rates for passenger cars are presented in Table 2-4, which is based on the Transportation and Traffic Engineering Hand Book (ITE, 1982). 
Table 2-4: Normal Acceleration and Deceleration Rates (ITE, 1982).

\begin{tabular}{cccccc}
\hline \multicolumn{2}{c}{ Speed Range } & \multicolumn{2}{c}{ Acceleration } & \multicolumn{2}{c}{ Deceleration } \\
$\mathrm{km} / \mathrm{h}$ & $\mathrm{mph}$ & $\mathrm{km} / \mathrm{h} / \mathrm{s}$ & $\mathrm{mph} / \mathrm{s}$ & $\mathrm{km} / \mathrm{h} / \mathrm{s}$ & $\mathrm{mph} / \mathrm{s}$ \\
\hline $0-24$ & $0-15$ & 5.3 & 3.3 & 8.5 & 5.3 \\
$0-48$ & $0-30$ & 5.3 & 3.3 & 7.3 & 4.6 \\
$48-64$ & $30-40$ & 5.3 & 3.3 & 5.3 & 3.3 \\
$64-80$ & $40-50$ & 4.2 & 2.6 & 5.3 & 3.3 \\
$80-97$ & $50-60$ & 3.2 & 2.0 & 5.3 & 3.3 \\
$97-113$ & $60-70$ & 2.1 & 1.3 & 5.3 & 3.3 \\
\hline
\end{tabular}

Driver acceleration and deceleration characteristics have been extensively analyzed in the past decades. It is observed that the acceleration and deceleration rates applied by drivers vary from driver to driver, and that the use of acceleration and deceleration depends on other factors such as the type of vehicle and the prevailing traffic and weather conditions. Due to the fact that drivers seldom apply extreme acceleration and deceleration rates in their normal driving, driver acceleration/deceleration behaviour is more closely related to the driver's preference rather than the characteristic of the vehicle. The following is a review of existing research pertaining to driver acceleration and deceleration behaviour.

Researchers hold different opinions on driver acceleration behaviour based on their own research. As shown in Figure 2-1, there are four kinds of typical acceleration models developed by previous researchers: constant acceleration model, two-phase model, linearly-decreasing acceleration model, and polynomial acceleration model. The constant acceleration model assumes that drivers maintain the average acceleration rate throughout 
the acceleration procedure. The two-phase acceleration model assumes that drivers use two acceleration rates during their acceleration procedure. More specifically, drivers would apply a higher acceleration rate at lower speeds and a lower acceleration rate at higher speeds. The linearly-decreasing acceleration model assumes that drivers will apply a highest acceleration rate at the initial speed of zero and reduce the acceleration rate linearly with the increase of speed till a constant speed is reached. The polynomial model provides a formation showing that the acceleration rates at the start and end of acceleration are zero and change smoothly in between.

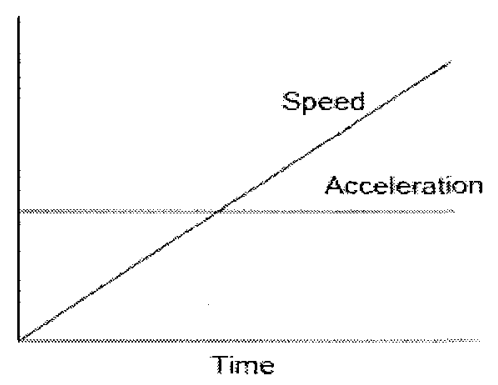

(a)

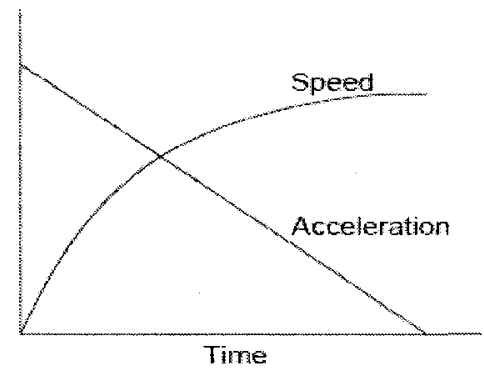

(c)

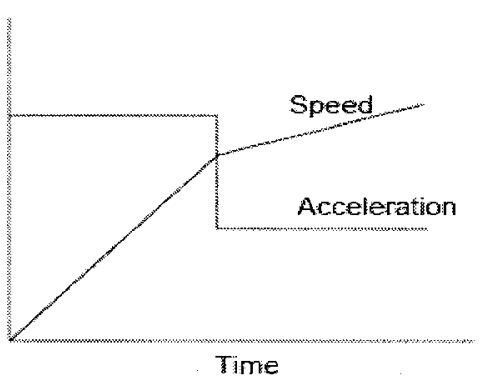

b)

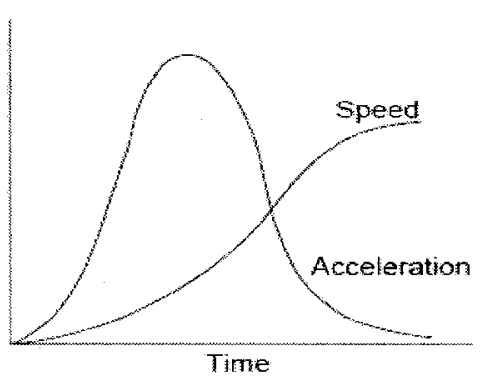

(d)

(a) Constant, (b) Two-phase, (c) Linearly-decreasing, and (d) Polynomial.

Figure 2-1: Four Common Acceleration Models (Wang et al., 2004).

Akcelik and Biggs (1987) studied vehicle acceleration characteristics from a stop condition in three types of locations (central business district, urban, and non-urban) in Australia. Speeds of 1,037 vehicles were measured second by second using an instrumented car. A polynomial model of acceleration was proposed, which gave a 
realistic reflection of zero jerks at the start and end of acceleration with an acceleration rate of zero. The relationship nearly appeared as a semicircle on an acceleration-versusspeed plot. The advantage of this study is that it had rich speed measurements, which recorded vehicle movements at evenly spaced time intervals. Therefore, the proposed model could be expected to give a better reflection of vehicle acceleration characteristics, especially at low speeds.

Long (2000) studied vehicle acceleration characteristics of both passenger cars and trucks from a standstill state by extensively reviewing pertinent literature. In addition, the author critically evaluated the acceleration parameters for design in the AASHTO Green Book. The author supported the theory that a linearly decreasing acceleration model better describes both maximum vehicle acceleration capabilities and actual motorist behaviour rather than a constant acceleration pattern. After comparing the acceleration parameters in the AASHTO Green Book with that in other publications that were based on thousands of field observations, Long claimed that design accelerations in the AASHTO Green Book deviate substantially from the observed accelerations.

Haas et al. (2004) evaluated driver deceleration and acceleration behaviour at stop sign-controlled intersections on rural highways using data collected during the Intelligent Cruise Control Field Operational Test in 1996. A total of 299 deceleration and 214 acceleration events were identified and, subsequently, a mathematical model was used to compute average rates of deceleration and acceleration for each of two events. The average rates were then compared for different subgroups which were based on initial speed, final speed, driver gender and type of street. The results showed that only the initial speed, from which deceleration began, had significant influence on the average 
deceleration rate, whereas other factors showed little influence on these average rates. Moreover, the observed rates of both deceleration and acceleration showed large variations in these average values. One conclusion drawn by the authors in this analysis was that the driver deceleration and acceleration behaviour cannot be effectively modeled by applying average rates of deceleration or acceleration.

Wang et al. (2004) verified the aforementioned four acceleration models for the allway stop-controlled locations using a database acquired from 100 vehicles equipped with Global Positioning System (GPS) and other instruments capable of recording location information and vehicle speeds at one-second intervals. The authors categorised the driving manoeuvres into straight and turning subsets. These two acceleration manoeuvres of straight and turning, according to their study, had no significant difference. Moreover, their verification revealed that the constant and two-phase acceleration models did not fit their data at all. In addition, the acceleration rates decreased with the increase of the average speed, but the relationship was not in a linearly decreasing manner. Therefore, the polynomial model was evaluated with more emphasis; however, the polynomial model proposed by Akcelik and Biggs (1987) did not fit the field acceleration rate either. As a result, new models were developed in interpreting the relationship between acceleration rate and average speed for straight and turning manoeuvres, respectively. The new models can be expressed as Equation 2.1:

$$
\sqrt{a}=a_{0}-a_{1} v
$$

Where $a$ is acceleration rate,

$v$ is speed, and

$a_{0}$ and $a_{1}$ are constant parameters. 
Wortman and Matthias (1983) studied driver deceleration behaviour associated with the traffic signal change interval in the Phoenix and Tucson areas. Time-lapse photography was used to observe driver behaviour at six intersections in daytime, and two of the six intersections were also observed using the same technology during night time. The authors analyzed driver behaviour parameters in terms of vehicle approach speed, average deceleration rate, and driver perception-reaction time based on the information extracted from the time-lapse film. The mean deceleration rates at their observed six sites ranged from 7.0 to $13.9 \mathrm{ft} / \mathrm{s}^{2}$, and the mean value for all observations was $11.6 \mathrm{ft} / \mathrm{s}^{2}$. Furthermore, the authors noted that the comparison of driver deceleration behaviours in daytime and night time did not yield a consistent result for the two locations. The finding of mean acceleration rate in the research was slightly greater than the comfortable deceleration of $10 \mathrm{ft} / \mathrm{s}^{2}$ suggested by ITE (1992).

Bennett and Dunn (1995) presented a study of driver deceleration behaviour in Auckland, New Zealand. They used the data acquired by seven pairs of axle detectors installed on a freeway exit ramp, at the end of which there was a traffic signal. The research was carried out for three classes of vehicles: (a) passenger car and small light commercial, (b) medium commercial, and (c) heavy commercial vehicle. Three models were developed for these three classes of vehicles, in a format as shown in Equation 2.2:

$$
S=S_{0}+a_{0} S_{0} t^{2}
$$

Where $S$ is the speed of the vehicle at time $t$ in $\mathrm{km} / \mathrm{h}$,

$S_{0}$ is the approach speed of the vehicle in $\mathrm{km} / \mathrm{h}$, and $a_{0}$ is the regression model coefficient. 
It was stated that vehicle deceleration was a function of approach speed and cumulative time. Furthermore, the authors found that vehicles decelerated over the same distance irrespective of the initial speed. More specifically, higher-speed drivers applied higher deceleration rates before approaching the stop in their research.

EI-Shawarby et al. (2007) studied driver deceleration characteristics at the onset of a yellow-phase transition using the data collected on a $3.5 \mathrm{~km}$ research road with a fourway signalized intersection. Sixty drivers were recruited in the experiment; their ages were between 20 and 82 and the numbers of male and female drivers were approximately equal. No other vehicle was allowed on the test road when the experiment was being conducted. During the experiment, participants were required to cruise at a speed of 72 $\mathrm{km} / \mathrm{h}$ while approaching the signalized intersection and the yellow phases were triggered at five different distances from the stop bar.

The authors found that drivers' mean deceleration rate increased with the decrease of available stopping distance. They also mentioned that a constant deceleration rate of 3.0 $\mathrm{m} / \mathrm{s}^{2}$ suggested by ITE (1992) cannot reflect the real deceleration rates, especially for the shorter time-to-stop line. In addition, it was found that male drivers applied slightly higher deceleration rates than female drivers. Moreover, the mean deceleration rate of younger drivers (under 40 years old) was significantly higher than the other age groups. Furthermore, the driver group of 60 years of age or older used greater deceleration rates than the medium age driver group (40 to 59 years old). Based on the results presented above, the authors suggested that a distribution of model parameters rather than constant values should be employed for the design of yellow phases. 
There were some limitations in the research by EI-Shawarby et al. (2007). One is that there were no surrounding factors affecting driver behaviour, which could therefore be far from the real driving conditions. Secondly, since the yellow phase was triggered much more frequently than the green phase in the experiment, drivers were more likely to expect a yellow phase and to make a corresponding stop after several test runs. Both of these two conditions suggest that the experiment environment was not a typical natural driving condition.

Gates (2007) studied the behaviour of 1001 drivers in the typical dilemma zone upstream of signalized intersections when the yellow phase started. Eight-millimetre analog video cameras were used to record factors of approaching vehicle movement including vehicle type, approach speed, distance upstream of the intersection at the start of the yellow signal, and brake-response time. From the collected data, deceleration rates and brake-response times were evaluated for first-to-stop vehicles and last-to-go vehicles. The deceleration rate for each individual driver was his/her average deceleration rate during the fully-stopped deceleration manoeuvre using the approach speed over the entire braking time. It was found that the $15^{\text {th }}, 50^{\text {th }}$, and $85^{\text {th }}$ percentile deceleration rates for first-to-stop vehicles were $7.2,9.9$, and $12.9 \mathrm{ft} / \mathrm{s}^{2}$, respectively. Moreover, deceleration rates were found to increase with the increase of approach speed and decrease with the increase of distance from the intersection. The variable of approach speed was found to present the heaviest effect on deceleration rate, followed by variables of distance to intersection and brake-response time.

In addition, the authors reported the deceleration behaviour of drivers in two speed groups: approach speeds greater than $40 \mathrm{mi} / \mathrm{h}$ and less than or equal to $40 \mathrm{mi} / \mathrm{h}$. Sixty- 
nine percent of the drivers in the first group used a deceleration greater than $10 \mathrm{ft} / \mathrm{s}^{2}$, which is the comfortable stopping deceleration rate specified by ITE (1992). In the second group, 26 percent of drivers used a deceleration rate greater than $10 \mathrm{ft} / \mathrm{s}^{2}$. Therefore, the authors suggested that the design values for comfortable deceleration at a signalized intersection should be based on approach speed rather than a single default value.

Liu et al. (2007) studied driver behaviour in dilemma zones at six signalized intersections, using the data of 1,123 drivers observed in the yellow phase. The driver population at each sample intersection was classified into three distinct driver groups: aggressive, conservative, and normal. Classifying the drivers was undertaken according to their responses to the yellow phase along with the relationship between their distanceto-stop-line $\left(x_{d}\right)$ and the critical distance $\left(d_{c}\right)$. Conservative drivers were those who took the stop action even though they could proceed through the intersection during the yellow phase $\left(x_{d}<d_{c}\right)$; aggressive drivers were those who passed the intersection even though they were quite far away $\left(x_{d}>d_{c}\right)$; and normal drivers were those who took the stop action when $x_{d}>d_{c}$ or the pass action when $x_{d}<d_{c}$. After comparing the key characteristics among the three driving groups, it was found that the aggressive group generally applied harder acceleration than other two groups while leaving intersections, and the conservative group usually applied harder deceleration than the other two groups while approaching intersections. The authors also noted that the aggressive-pass group usually applied an approaching speed about 10-20 percent higher than the average traffic flow speed, while the conservative-stop group usually applied an approaching speed of about 10-15 percent lower than the average traffic flow speed. 


\subsection{Summary}

This chapter presented the review of existing literature covering the relationship between speed and road safety, examination of driver speed choice, and driver acceleration and deceleration characteristics. Numerous researchers and professionals have made important efforts in improving the mobility, efficiency, and safety of the ground transportation system, by examining traffic crash inducements, developing highway design technology, investigating driving characteristics, and improving vehicle quality. However, there are still many issues that need to be addressed with consideration of the current traffic safety realities in Canada. These issues may be related to any of the three key components (the driver, the roadway, and the vehicle) of transportation system, or with their interaction.

Although surrogate safety measures in terms of improvements in the roadway design and development of vehicle safety devices can increase transportation safety, positive changes in driver behaviour should be developed as well since unsafe driving manners are a substantial contributing factor in a large number of road accidents. Undoubtedly, extensive attempts have been undertaken in previous studies which provide better understanding of driving behaviour and the related aspects. However, some limitations in previous research can still be highlighted as follows:

Study scope: Almost all previous studies focused on one road type, especially twolane rural highways, and the majority of previous research involved one specific location such as horizontal curves or intersections. Little research is available to draw a big picture of driver behaviour on different road classifications. 
Data collection: Although any measurement has some level of error in measuring drivers' speeds, some data collection techniques have systematic errors that tend to create some bias in the data. For example, the speed measurement in many previous studies relied on the use of radar guns, which reportedly produced a significant drop in speeds averaging $7 \mathrm{~km} / \mathrm{h}$ in a Canadian study (Hassan, 2004).

Outdated data: Capabilities of current vehicles are considerably different from those in the past decades due to the great development of the vehicle industry. Meantime, highway design and construction technologies have been improved significantly and driver attitude may have changed with the development of society. This means that the reliability of relatively old data may be compromised for addressing today's issues.

Applicability: It is believed generally that driver behaviour varies from one region to another due to differences in traffic composition, highway features, and levels of traffic enforcement. Since research pertaining to driver behaviour on Canadian roads is relatively small and it is questionable to apply the results of research overseas directly to Canadian cases, it is necessary to perform new research based on local information.

Due to these limitations, it is necessary to undertake a comprehensive investigation of contemporary driver behaviour on Canadian roads. This research studies driver speed and acceleration/deceleration behaviour using the real driving data collected on the four most common road classes in Eastern Ontario. The results should provide a good insight of driver behaviour on Canadian roads for individual drivers and interested agencies. As a result, it is expected that any necessary education could be developed to rectify driver 
behaviour to enhance road safety and, additionally, to reduce fuel consumption and exhaust emission on our roads. Additionally, it should be helpful for transportation professionals to build a more compatible transportation system on the basis of awareness of driving behaviour on Canadian roads. 


\section{CHAPTER 3: DATA COLLECTION}

In order to fulfill the objectives of this research, an experiment was undertaken to measure driver behaviour on different road classes under real driving circumstances. The pre-designated test route covered four road types: urban freeway, two-lane rural highway, rural freeway, and urban/suburban road. Data collection was conducted on the test route from August to October 2005 using a test vehicle. The test vehicle was equipped with a variety of instruments capable of recording the vehicle's kinematic and dynamic parameters, vehicle positions, and traffic conditions outside of the test vehicle.

This chapter presents the data collection scheme that gives the whole picture of the data collection procedure, followed by the selection of the test route and the recruitment of volunteer drivers for this experiment. This chapter also includes the introduction of the equipment used in the experiment and the description of the data collected by each piece of equipment. In addition, the method of equipment synchronization is also explained in this chapter.

\subsection{Data Collection Scheme}

The data collection process was composed of two parts, one was the preparation before field experiment and the other was the actual data collection. Experiment preparation included determination of the test route, installation and calibration of the equipment, and recruitment of volunteer drivers. In each data collection run, one of the volunteer drivers drove the test vehicle along the selected test route. Meanwhile, two other experimenters were responsible for ensuring that the instruments were working

properly and were also operating some instruments like the laser guns. Drivers' 
performance during the test was recorded in terms of multiple measurements, including instantaneous speed of the vehicle and use of throttle, braking, and steering by the driver. The positions of the vehicle were measured by a GPS receiver. In addition, a diversity of the vehicle's operation parameters (e.g., engine revolution, voltage, etc.) was recorded by a Vehicle Interface Unit (VIU), which was also used to measure the vehicle's speed. After each test run, the raw data collected by the various instruments, except those collected by the VIU, were immediately downloaded onto a laptop. The VIU data were dynamically extracted using the wireless technology (Wi-Fi). The test runs were all arranged in daytime and under normal weather conditions (e.g., without rain, fog, etc.).

In data collection, the only instruction given to the drivers was to drive according to their usual driving habits. Before the start of data collection, the drivers were allowed to drive for 20-30 minutes to become familiar with the vehicle. The commencement of data collection was intentionally not announced to the drivers.

\subsection{Selection of Test Route}

Before the commencement of the actual test runs, a test route was selected in Eastern Ontario to cover four common road types: urban freeway, two-lane rural highway, rural freeway and urban/suburban road. For practical purposes, it was selected in the vicinity of the City of Ottawa. The test route consisted of seven roads and four freeway interchanges. The total length of the test route was approximately $110 \mathrm{~km}$, and the average time elapsed on the test route was about 90 minutes. Table 3-1 shows the names of the seven roads, the segment length of each road, and the corresponding road types in the test route along the direction of test run. 
Table 3-1: Seven Roads and Their Segment Lengths in the Test Route.

\begin{tabular}{|l|l|r|}
\hline Road Type & \multicolumn{1}{|c|}{ Road Name } & Length (m) \\
\hline Interchange Entry Ramp & Bronson Ave. to Highway 417 & 280 \\
\hline Urban Freeway & Highway 417 & 24,885 \\
\hline Interchange Exit Ramp & Highway 417 to Highway 7 & 2,254 \\
\hline \multirow{3}{*}{ Rural Highway } & Highway 7 (Provincial Road 7) & 9,095 \\
\cline { 2 - 4 } & Regional Road 3 (Dwyer Hill Rd.) & 22,862 \\
\cline { 2 - 4 } & Regional Road 6 (Roger Stevens Dr.) & 19,122 \\
\hline Interchange Entry Ramp & Regional Road 6 to Highway 416 & 365 \\
\hline Rural Freeway & Highway 416 & 16,473 \\
\hline Interchange Exit Ramp & Highway 416 to Regional Road 12 & 620 \\
\hline \multirow{2}{*}{ Urban/Suburban Road } & Regional Road 12 (Fallowfield Rd.) & 8,523 \\
\cline { 2 - 4 } & Regional Road 73 (Prince of Wales Dr.) & 6,766 \\
\hline \multicolumn{2}{|c|}{ Total Length: 112,245 } \\
\hline
\end{tabular}

As shown in Table 3-1, Highway 417 is an urban freeway; Highway 7, and Regional Roads 3 and 6 are undivided two-lane rural highways; Highway 416 is a rural freeway; and Regional Roads 12 and 73 are urban/suburban roads. Highway 7, Regional Roads 3 and 6, and Highway 416 traverse rural areas where light traffic is expected. The section of Highway 417 in this study connects central Ottawa and Kanata. Regional Roads 12 and 73 are located in or near the City of Ottawa, where moderate to heavy traffic volumes are prevalent. 


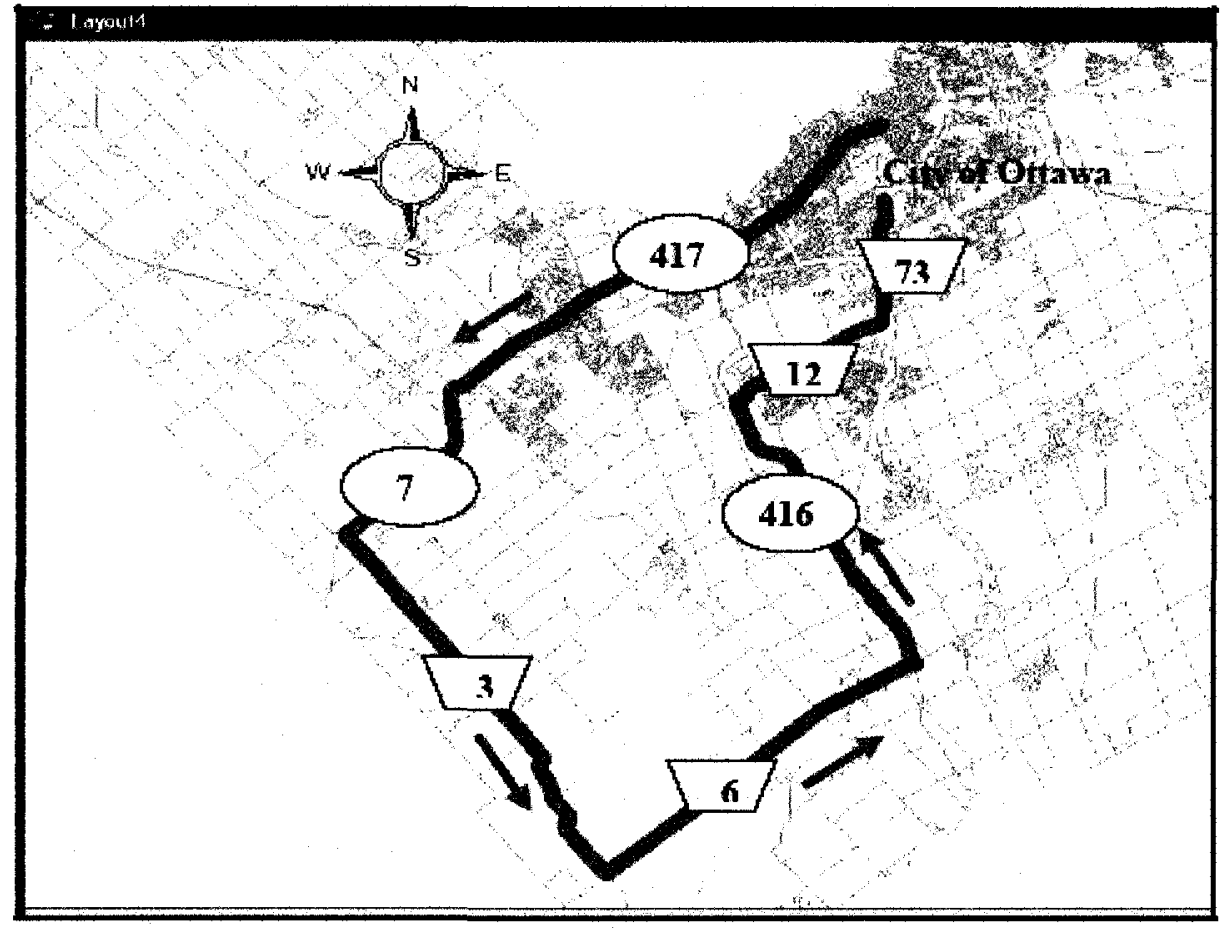

Figure 3-1: Map of the Test Route

Figure 3-1 illustrates the map of the related road network, in which the test route is highlighted as a bold line. The highway number of the seven involved roads is displayed on corresponding road sections and the arrows represent the driving direction of the test vehicle. As shown in this figure, the direction of the test runs is depicted as follows:

- Highway 417: from Bronson entry ramp to Exit No. 145 of Highway 417.

- Provincial Route 7: from Exit No. 145 of Highway 417 to the intersection with Regional Route 3. This intersection was traffic-light-controlled.

- Regional Route 3: from the intersection with Provincial Route 7 to the intersection with Regional Route 6. This intersection was stop-sign-controlled.

- Regional Route 6: from the intersection with Regional Route 3 to the interchange with Highway 416. 
- Highway 416: from the interchange with Regional Route 6 to Exit No. 66 of Highway 416.

- Regional Route 12: from Exit No. 66 of Highway 416 to the intersection with Regional Route 73. This intersection was traffic-light-controlled.

- Regional Route 73: from the intersection with Regional Route 12 to the intersection with Hog's Back Road.

\subsection{Driver Recruitment}

In order to obtain real driving data, volunteer drivers were recruited to participate in the test driving. The selected driver sample should be representative of the majority of driver population in Ontario. According to an annual report of MTO (2006), G and G2licensed drivers in 2004 were of 74.81 and 7.64 percent of all Ontario drivers, respectively. Namely, the G-licensed drivers account for more than 90 percent of passenger car drivers. Additionally, for safety reasons, a G license indicates that the driver has been officially proven to have enough driving experience in high-speed environments since the test runs in the experiment involved driving on high speed freeways. So the selection of volunteer drivers was limited to G-licensed drivers. Thirty qualified drivers were recruited as a driver sample for the experiment to meet the requirement of the minimum sample size for statistics. The sample covered a wide range of driver ages from 20 to 50 years and driving experience from less than 5 years up to more than 20 years.

Before the start of each test run, the experiment, including the test route and the instruments, was introduced to the driver. This explanation ensured that the driver had no 
confusion about their task and was comfortable with the instruments beside him/her. In addition, each driver was asked to answer a questionnaire regarding his/her basic information and self-reported general driving pattern according to five general categories: extremely cautious, moderately cautious, slightly cautious, slightly aggressive, and aggressive. After completion of one test run, the driver was asked to fill another form regarding his/her experience during the test. One question was whether he/she was comfortable during the test, for which all drivers gave a positive answer.

\subsection{Experiment Equipment}

The test vehicle used in the experiment was a Ford Windstar minivan manufactured in 2003 and owned by Carleton University. The test vehicle was equipped with a variety of instruments, including a Corsa Data Acquisition Box, a GPS receiver, two laser guns, a video camera, and a Vehicle Interface Unit (VIU). The instruments, except the VIU, belonged to Carleton University, while the VIU was manufactured and owned by the Netistix Technologies Corporation. Therefore, in the later subsections, the VIU and the rest of the equipment are introduced separately under two nominal categories: Netistix System and Carleton System.

These instruments were used to record a diversity of data. The Corsa Data Acquisition Box was used to measure instantaneous speed of the vehicle; the use of throttle, braking and steering; and the lateral and longitudinal acceleration of the vehicle. The Global Positioning System (GPS) receiver was used to record the vehicle trajectory in the format of three-dimensional coordinates: easting, northing, and elevation. The laser guns were used to measure the distances between the test vehicle and both of the leading and 
following vehicles in the same lane. The video camera was used to record driver's front view during the test. The VIU was connected to the on-board diagnostics (OBDII) and was used to detect multiple measurements. Table 3-2 shows a summary of the instruments used in the experiment and the type of data measured by each instrument. More details regarding each individual instrument and its collected data are described in the following sections.

Table 3-2: Summary of the Experimental Instruments and Data Types.

\begin{tabular}{|c|c|c|c|}
\hline \multicolumn{2}{|c|}{ Carleton System } & \multicolumn{2}{|c|}{ Netistix System } \\
\hline Data Type & Instrument & Data Type & Description \\
\hline Speed & \multirow{5}{*}{$\begin{array}{l}\text { Corsa Data } \\
\text { Acquisition Box }\end{array}$} & Speed & Instantaneous speed \\
\hline Throttle & & Throttle & Throttle position \\
\hline Braking & & Odometer & Odometer reading \\
\hline Steering & & Vehicle states & $\begin{array}{l}\text { Vehicle off, stationary } \\
\text { or moving }\end{array}$ \\
\hline Acceleration & & VIU events & $\begin{array}{l}\text { Wi-Fi connection and } \\
\text { disconnection, } \\
\text { engine on and off }\end{array}$ \\
\hline $\begin{array}{l}\text { Vehicle path } \\
\text { (coordinates) }\end{array}$ & GPS Receiver & Engine data & $\begin{array}{l}\text { Engine run time, } \\
\text { idle time, } \\
\text { revolution } / \mathrm{min} \text {, coolant } \\
\text { temperature }\end{array}$ \\
\hline $\begin{array}{l}\text { Distance from } \\
\text { vehicle in front }\end{array}$ & Laser Gun & Voltage & $\begin{array}{l}\text { Voltage of startup, } \\
\text { during running, } \\
\text { and engine off }\end{array}$ \\
\hline $\begin{array}{l}\text { Video } \\
\text { (front view) }\end{array}$ & Video Camera & MIL status & $\begin{array}{l}\text { Malfunction Indicator } \\
\text { Light (MIL) }\end{array}$ \\
\hline
\end{tabular}




\subsection{Carleton Data Collection System}

\subsection{1.a Corsa Data Acquisition Box}

The Corsa Box is a data acquisition system that records vehicle kinematic and dynamic characteristics through a variety of sensors connected to corresponding parts. The Corsa Box was configured beforehand to allow it to record a set of readings every 0.2 seconds. The time stamps recorded in the Corsa Box were in a cumulative manner beginning with zero and cumulating by 0.2 seconds. The collected raw data were downloaded to a laptop after completion of each run, and were then processed using a proprietary software to produce easy-to-read files, as specified by the user. The following are the types of data measured by the Corsa Box.

a. Use of Throttle, Braking and Steering:

The use of throttle, braking, and steering was measured using three sensors connected to the moving part of the brake pedal, fuel pedal, and steering wheel of the test vehicle, respectively. The readings reflected how far the three devices moved from its original position. The three kinds of readings are shown in units of percent, and a sign before the steering value represents the turning direction of the steer as shown in the example (Figure 3-3).

\section{b. Lateral and Longitudinal Accelerations:}

The lateral and longitudinal accelerations were measured using two accelerometers within the data acquisition box. The accelerations were recorded in units of $g(9.81$ $\left.\mathrm{m} / \mathrm{sec}^{2}\right)$ 


\section{c. Instantaneous Speed:}

The instantaneous speed was measured using a magnetic speed sensor attached to the left rear wheel of the test vehicle. The speed sensor is a switch that responds whenever a magnet passes by its face. In the sensor installation, four magnets were mounted on the moving part of the wheel, and the speed sensor was assembled facing the magnets on a bracket fixed on the vehicle body. When the wheel turned, the sensor detected the signal when it passed by each of magnets and the output signals were transmitted to the data acquisition box through a connecting cable. The Corsa system then converted the signals to a speed value in units of $\mathrm{km} / \mathrm{h}$. The magnitude of the speeds depended on the changing frequency of the signals. The more frequent change of the signals, the faster the speed.

Calibration regarding the conversion of signal pulses into speed values was carried out beforehand in the configuration file of the Corsa system. The calibration procedure contained the following steps to obtain the required parameter:

- Determination of the tire rolling circumference (inch).

- Computation of number of tire revolutions per mile using 63,360 (inch/mile) over the measured tire rolling circumference.

- Calculation of signal pulse per mile using the number of tire revolutions per mile to multiply the number of magnets mounted on the wheel.

- Determination of the parameter of "Scale" (Hz/mph) for the configuration file. The scale was obtained using pulse per mile over 3600 (second/hour), and then it was inputted in the configuration file to finish the calibration. 
Figure 3-2 shows the test vehicle; the Corsa Box; the speed sensor; and sensors for measuring throttle, braking, and steering, respectively. Figure 3-3 shows an example of Corsa output data file in Excel format.

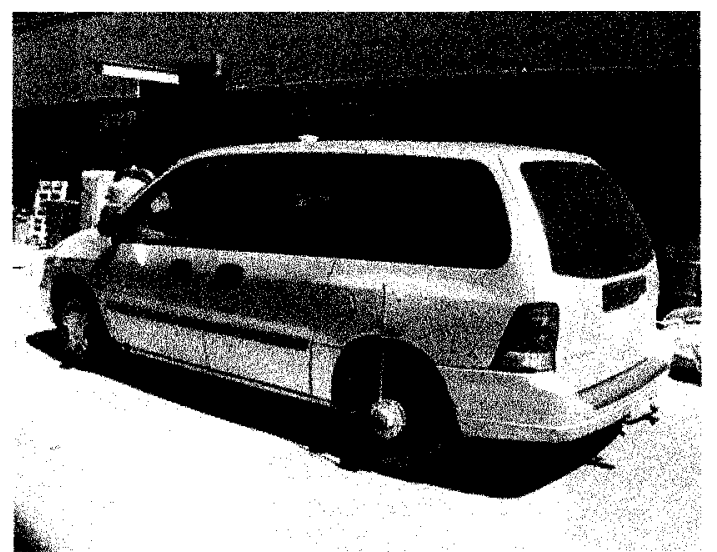

(a) Test Vehicle

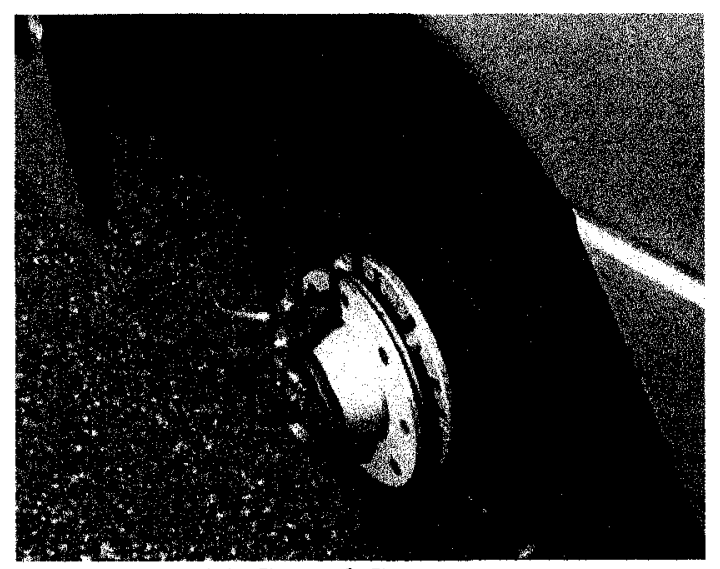

(c) Speed Sensor

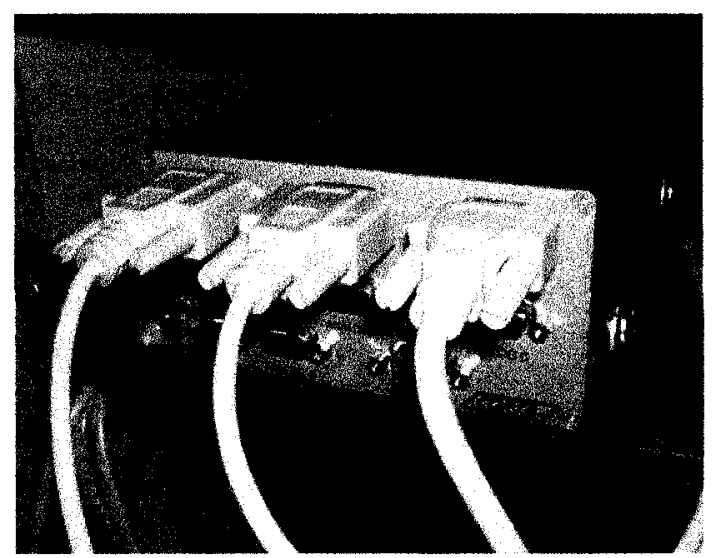

(b) Corsa Data Acquisition Box

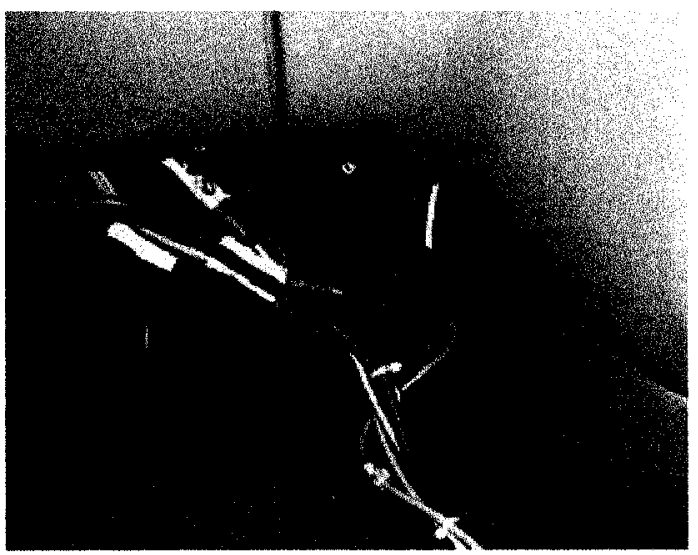

(d) Sensors for Measuring Throttle, Braking, and Steering

Figure 3-2: Test Vehicle and the Corsa Data Acquisition System. 


\begin{tabular}{|c|c|c|c|c|c|c|c|c|c|c|}
\hline \multicolumn{11}{|c|}{ 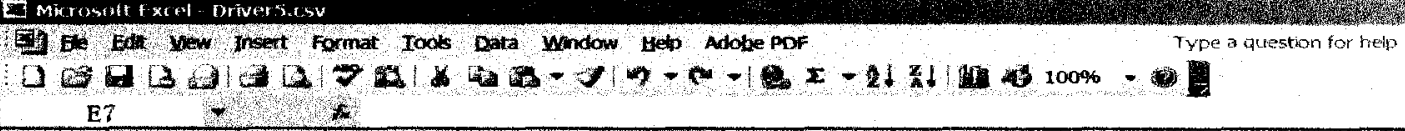 } \\
\hline & A & $\mathrm{B}$ & C & $\mathrm{b}$ & W & F & G & $\sqrt{1}$ & I & 23 \\
\hline 1 & \multicolumn{10}{|c|}{ Driver5. dat start $=0.000$ end $=4985.600$} \\
\hline 2 & Time & Lateral & LonG & Box Temp & RPM & peed & Brake & Throttle & Steering & \\
\hline 3. & 0 & -0.207 & 0.136 & -16.1 & 0 & 73.83 & 0.429 & 55.714 & -11.505 & \\
\hline 4 & 0.2 & -0.117 & 0.129 & & & 49.851 & 0.286 & 55.714 & -7.742 & \\
\hline 6 & 0.4 & -0.048 & 0.141 & & & 50.983 & 0.286 & 55.714 & -6.129 & \\
\hline 6 & 0.6 & -0.008 & 0.134 & & & 51.163 & 0.286 & 55.429 & -5.376 & \\
\hline U. & 0.8 & -0.031 & o. 129 & & & 51.98 & 0.286 & 50.429 & -4.516 & \\
\hline 8 & 1 & -0.026 & 0.129 & -16.1 & & 53.077 & o. 286 & 54.857 & -3.978 & \\
\hline 9 & 1. 2 & -0.024 & o. 139 & & & 53.393 & 0.286 & 54. 571 & -3.656 & \\
\hline 10 & 1. 4 & -0.019 & 0.111 & & & 54.237 & 0.286 & 54.857 & -2.151 & \\
\hline 11 & 1.6 & 0.01 & 0.125 & & & 55.319 & 0.286 & 54.857 & -0.968 & \\
\hline 12 & 1.8 & 0.027 & 0.139 & & & 56.058 & 0.286 & 54.857 & -1.183 & \\
\hline 13 & 2 & 0.008 & 0. 129 & -16.1 & & 56.159 & 0. 286 & 54.857 & -1.075 & \\
\hline 14 & 2. 2 & 0.001 & 0.129 & & & 57.232 & o. 286 & 54.857 & -0.86 & \\
\hline 15 & 2. 4 & 0.001 & o. 129 & & & 58 & 0.286 & 55.143 & -0.108 & \\
\hline 16 & 2.6 & 0.008 & 0.125 & & & 58.565 & 0.286 & 53.714 & 0.968 & \\
\hline 17 & 2.8 & 0.039 & 0.125 & & & 59.06 & o. 286 & 50. 571 & 1.935 & \\
\hline 18 & 3 & 0.058 & 0.12 & -16 & & 59.923 & 0.286 & 50.857 & 3.333 & \\
\hline 19 & 3.2 & 0.086 & 0.111 & & & 60.037 & 0. 286 & 52 & 4. 731 & \\
\hline 20 & 3. 4 & 0.126 & 0.115 & & & 60.909 & 0.143 & 52.857 & 5. 269 & \\
\hline 21 & 3.6 & o. 143 & 0.113 & & & 62.011 & o. 143 & 51.429 & 5. 806 & \\
\hline
\end{tabular}

Figure 3-3: Example of Corsa Output Data.

\subsection{1.b GPS System}

The Global Positioning System (GPS) is capable of determining the geographic location of an object if it is equipped with a receiver to receive signals from satellites in space. However, the GPS data may be affected by several error sources. These sources of errors include Selective Availability (SA), Geometric Dilution of Precision (GDOP), and a range of other errors including satellite's internal and external errors, receiver errors, atmospheric errors, and multipath errors (Sin, 2001). In this experiment, the Differential Kinematic GPS survey method was used, which could minimize various errors during the GPS observation and thus improve the accuracy of measurements.

For employing the Differential Kinematic GPS method, two sets of GPS receivers (Leica SR530 Geodetic RTK Receivers) were used in the experiment. One was a static receiver placed on a control station whose coordinates were known, while the other was a rover receiver, whose antenna was mounted atop the test vehicle to capture the vehicle 
position during the test runs. The control station was chosen close to the centre of test route. Due to the relatively short distance between the static receiver and rover receiver, the errors imposed on both of the receivers were considered almost identical. The readings of the rover receiver were then calibrated, using the errors detected by the static receiver, in the software SKI-Pro (Leica Geosystems Inc., 2001). This software can also transform the original coordinates into the local coordination system; this capability provides facilities for further integration of the GPS data with the highway centreline in GIS environments.

In the experiment, the GPS receivers were set to record 5 readings per second. The vehicle instantaneous coordinates were recorded with a time stamp for each reading. Figure 3-4 shows the static GPS receiver on the control station and the rover GPS receiver on the test vehicle. Figure 3-5 presents an example of GPS output data.

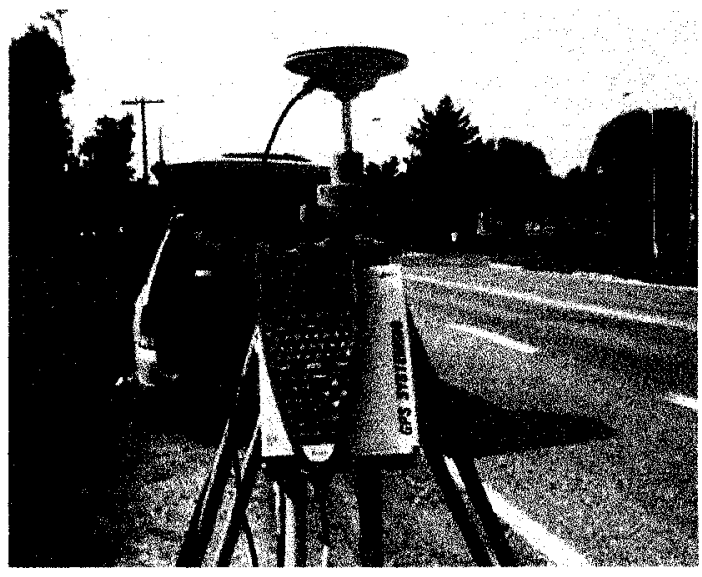

(a) Static GPS Receiver.

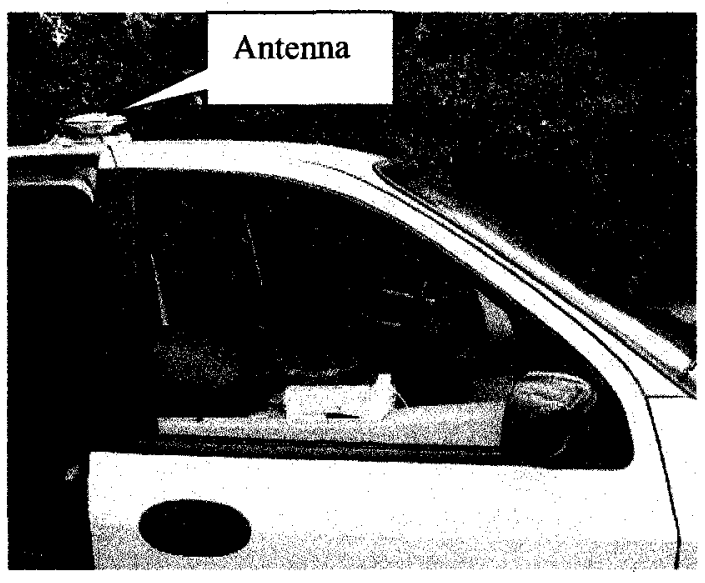

(b) Rover GPS Receiver.

Figure 3-4: GPS Receivers. 


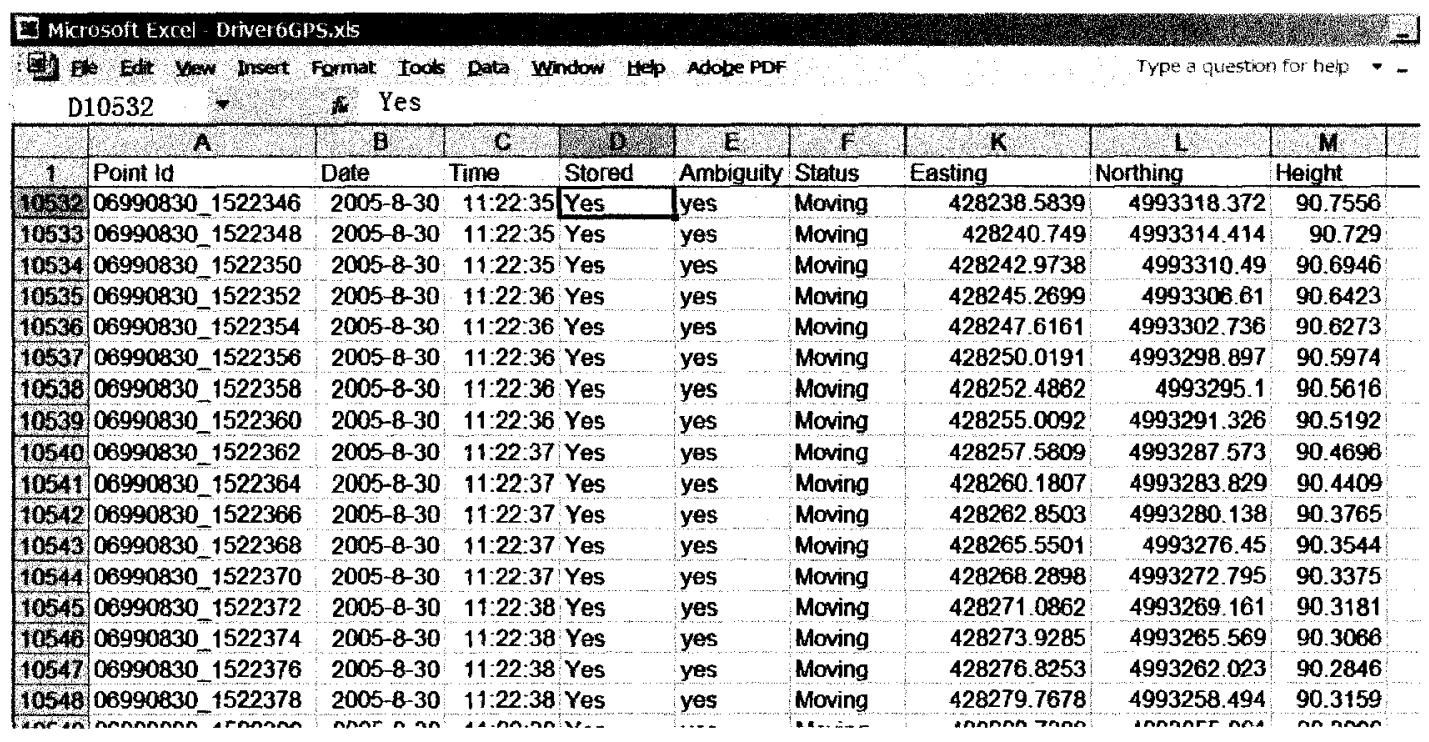

Figure 3-5: Example of GPS Output Data.

\subsection{1.c Laser Guns}

Two laser guns (SpeedLaser ${ }^{9}$ ) were mounted in the test vehicle; one was set at the front as shown in Figure 3-6 and the other was set at the back. The laser guns were used to measure the speed difference and the distance between the test vehicle and the leading and following vehicle in the same lane. The distance range of the laser guns is up to 7,000 feet $(2,133$ meters $)$ and their speed range is from $10 \mathrm{mph}(16 \mathrm{~km} / \mathrm{h})$ to $200 \mathrm{mph}(320$ $\mathrm{km} / \mathrm{h}$ ). The recording elements in this data file contained the distance in units of meter and relative speed in units of $\mathrm{km} / \mathrm{h}$ if detected successfully, under the time tag when the laser gun was triggered. The distance between the vehicles could be used to identify whether the speed of the test vehicle was under free-flow conditions or constrained conditions. 


\subsection{1.d Video Camera}

A video camera was installed to record driver's front view during the test runs (Figure 3-10). The videos provided important evidence of any occasional events that might have influenced the driver's behaviour during the test course.

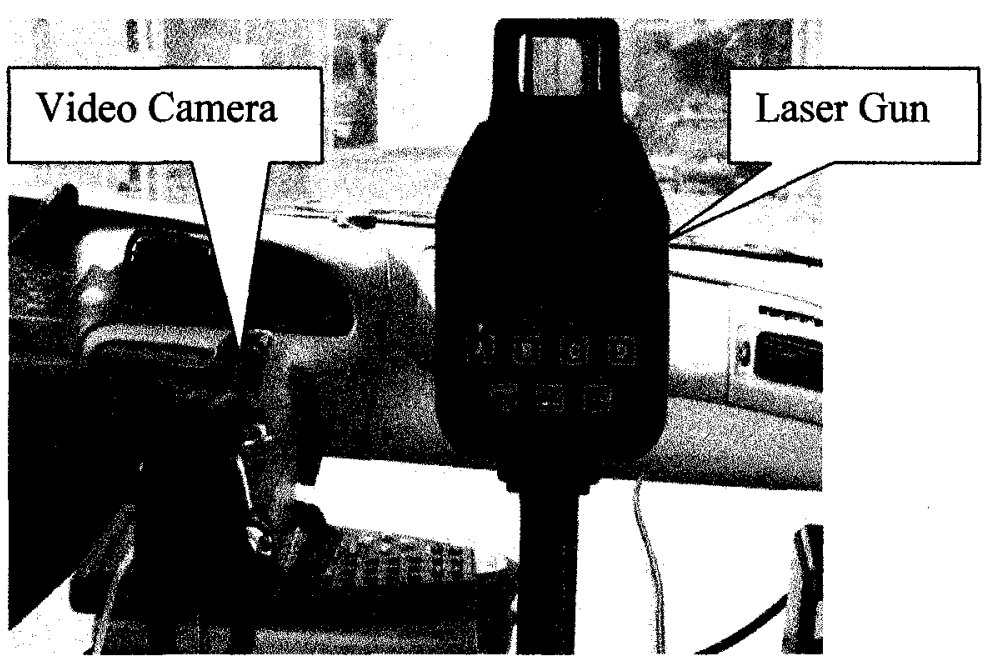

Figure 3-6: Laser Gun and Video Camera.

\subsubsection{Netistix Data Collection System}

In addition to the above devices belonging to Carleton University, another data collection device was a cabled Vehicle Interface Unit (as shown in Figure 3-7), which is a product of Netistix Technologies Corporation. The VIU is a below-dash-mounted device that plugs directly into the on-board diagnostics (OBDII) port and collects data from the engine control unit (ECU). The OBDII is provided in all cars built since 1996. The onboard diagnostics systems are able to provide engine control and monitor parts of the chassis, body and accessory devices, as well as the diagnostic control network of the car. 


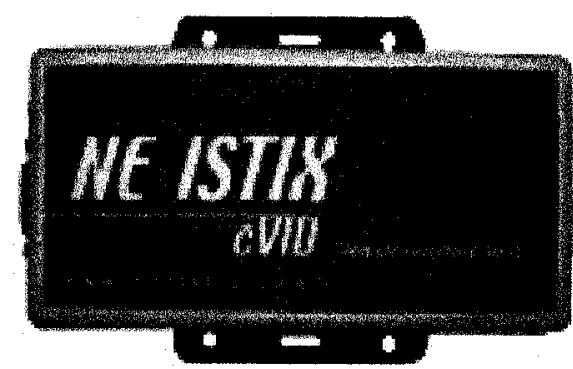

(a) Vehicle Interface Unit.

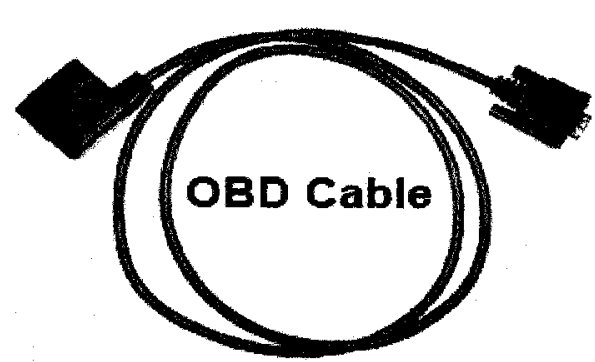

(b) OBD Cable.

Figure 3-7: Vehicle Interface Unit.

The VIU can extract the vehicle's operational data in three layers. One is basic logistical data including odometer, battery, engine coolant, and malfunction indicator light (MIL) status. The second type is in-depth technical indicators including misfires, oxygen sensor status, fuel trim, etc. Another type of data is the vehicle's kinematic and dynamic characteristics such as speed and use of throttle.

Recorded operational data were stored in the VIU internal disk. The data in the VIU can be seamlessly transferred by utilizing the wireless technology (Wi-Fi) from the vehicle to the VIUPoint Authentication Server, which has a complete wireless access point typically installed at convenient places such as fuelling depots, vehicle service areas, and fleet yards. The VIUPoint is used to acquire data dynamically from the VIU as well as download programming information to the VIU. The VIUPoint, in turn, delivers acquired in-field data to the OverVIU Information Manager, which gathers and analyses the collected data and presents reports to users and interested third parties.

Additionally, the VIU allows a GPS receiver to be plugged into its existing serial port. This allows the vehicle's location to be tracked using the GPS receiver. The GPS location information can also be stored in the VIU and conveniently downloaded to the VIUPoint 
as other data. In this experiment, no GPS receiver was installed onto the VIU because more accurate GPS receivers had already been employed as mentioned above.

In this study, the raw data extracted from the Netistix system were written in 17 separate files in the ASCII format. In each recording item, the recorded data was tagged with the corresponding time of day. An example of the VIU output data is shown in Table 3-3 together with a simple description of each data item. In the interests of this research, some data types are omitted in the example.

Table 3-3: Example of VIU Output Data.

\begin{tabular}{|c|c|c|c|}
\hline \multirow{2}{*}{ File } & \multicolumn{2}{|r|}{ Data Format } & \multirow{2}{*}{ Description } \\
\hline & Recorded Value & Time of Day & \\
\hline Speed & 41 & 26-Aug-2005 11:52:10 & $\mathrm{V}=41 \mathrm{~km} / \mathrm{h}$ \\
\hline $\begin{array}{l}\text { Throttle } \\
\text { Position }\end{array}$ & 24.3 & 26-Aug-2005 11:47:00 & $\mathrm{TP}=24.3 \%$ \\
\hline Odometer & 9708.1 & 07-Sep-2005 15:59:44 & $\begin{array}{l}\text { Odometer = } \\
9708.1 \mathrm{~km}\end{array}$ \\
\hline \multirow{3}{*}{$\begin{array}{l}\text { Vehicle } \\
\text { States }\end{array}$} & 87 & 27-Sep-2005 9:23:51 Moving & $\begin{array}{l}\text { Before } 9: 23: 51 \\
\text { the car moved for } \\
87 \text { seconds }\end{array}$ \\
\hline & 24 & 27-Sep-2005 9:24:15 Idle & $\begin{array}{l}\text { The car idled from } \\
9: 23: 51 \text { to } 9: 24: 15 \\
\text { for } 24 \text { seconds }\end{array}$ \\
\hline & 748 & 27-Sep-2005 9:36:43 Off & $\begin{array}{l}\text { And then the car } \\
\text { kept off for } 748 \\
\text { seconds. }\end{array}$ \\
\hline \multirow{4}{*}{$\begin{array}{c}\text { VIU } \\
\text { Events }\end{array}$} & Engine Started & 07-Sep-2005 10:11:18 & Engine was on \\
\hline & WIFI Connect & 07-Sep-2005 10:12:48 & $\begin{array}{l}\text { Wi-Fi connection } \\
\text { started }\end{array}$ \\
\hline & WIFI Disconnect & 07-Sep-2005 10:12:52 & $\begin{array}{l}\text { Wi-Fi connection } \\
\text { stopped }\end{array}$ \\
\hline & Engine Stopped & 07-Sep-2005 10:17:03 & Engine was off \\
\hline
\end{tabular}




\subsection{Equipment Coordination}

Since multiple pieces of equipment were used during the data collection, it was important to synchronize all equipment before the start of each test run in order to facilitate future data analysis. The VIU has an internal clock that could automatically record the time of day for every recording item. The GPS receivers and laser guns were set to the same exact timing with the laptop, which was used to store the raw data, before each test run. After completion of data collection each time, the collected data by the Carleton System (Corsa Box, GPS, and laser guns) were downloaded to the laptop. The VIU data was extracted and provided by Netistix Technologies Corporation after completion of the 30 test runs. 


\section{CHAPTER 4: DATABASE CONSTRUCTION AND COMPARISON OF TWO MEASURES}

In addition to the data of driving characteristics and the vehicle trajectory attained using the diverse instruments in the experiment, the highway network map related to this research was obtained from the City of Ottawa. The highway network map, which is in GIS environments, contains the road features in the Ottawa area such as road names, segment length of roads, and spatial attributes of roadway centerlines. Database construction was to relate the observed data to the roadway centerlines for each driver, by linking the actual vehicle travel trajectory to highway centerline data.

Moreover, in order to understand better the measurements of the two systems, Carleton's and Netistix's, comparison of the measurements of the two systems was conducted for their common data. Although the VIU recorded multiple types of data, only instantaneous speed and throttle position are the common ones with that of Corsa Box in this experiment. Furthermore, these two types of data are the ones related to driver behaviour, which is the focus of this research. Therefore, the speed data and throttle data collected by the two systems were compared.

\subsection{Database Preparation}

To prepare the database, a stepwise procedure was performed in Arcview 3.2a and Excel to execute the data integration. Nie (2006), using the same raw data, explained the methodology and procedure in details in combining the speed data collected by the Corsa Box and the vehicle trajectory with the roadway centerline. Since this research uses the intermediate results of data integration processed by Nie (2006), his related efforts are 
briefly introduced, followed by the additional steps performed in the context of this research to integrate the speed data collected by the VIU into the database.

\subsubsection{Introduction of Arcview}

In this study, Arcview 3.2a is an important software that was used in combining the data from different sources. Arcview is a geographic information system software made by the Environmental Systems Research Institute (ESRI) that is capable of visualizing, exploring, analyzing, and managing data geographically. It supports a variety of data formats such as vector data (e.g., shape files), raster data (image files) and tabular data (e.g., dBase files). The shape files are useful in displaying objects in points, lines, and polygons in the view and the tabular data can be used to store and describe the attributes of the objects. A shape file and its attribute table are interactively correlated, thus any change in the shape file could be updated easily in its corresponding attribute table and vice versa. Moreover, Arcview is capable of reading and processing the tabular data from external sources in many data formats such as dBase files, ARC/INFO tables, and comma delimited or tab delimited files. The tabular data can also be exported to various data types for application of other software.

\subsubsection{Procedure of Database Construction}

Integration of the Corsa data and GPS data was conducted for each individual driver by Nie (2006) in two steps:

1. The Corsa data and the GPS data were loaded in Arcview 3.2a. 
2. The Corsa data and the GPS data were joined to produce a new dataset using the "Table Join" function in Arcview. The join was based on the time stamps in their common fields in these two datasets.

The created dataset contained the Corsa measurements (e.g., speed, throttle, and so on) and GPS measurements (e.g., coordinates), referenced by the time.

The datasets created by Nie (2006) did not include the speed data collected by the VIU. Therefore, additional steps were undertaken to integrate the VIU data with the created datasets. It should be noted that the VIU was designed to record basically vehicle speed every second. However, in order to use effectively the internal memory of the VIU, one of the design features is that the VIU does not necessarily record the instantaneous speed for the periods of time it remains constant. Another situation was that the data collection by the VIU was independent of the operation of the experimenters. The VIU constantly recorded the vehicle operational data as long as it was installed in the vehicle.

Integration of the VIU speed data into the database involved the following steps, continuing after Steps 1 and 2 above:

3. The VIU speed data for each driver were sorted and separated into individual files according to their experiment time using Excel.

4. The dataset created by Nie (2006) as completion of Steps 1 and 2 was exported from Arcview 3.2a, and then filtered to match the VIU data based on their common time stamps for each driver in Excel. The filtered data were thereafter saved in a separate file, which contained the Corsa data, VIU data, and GPS data. 
5. The centerlines of all roads on the driving route were loaded into Arcview 3.2a and were then converted from line shape into point shape and the coordinates of each point were produced in its attribute table.

6. The created dataset produced in step 4 was loaded into Arcview 3.2a in the same file as the route centerline data. This dataset was designated as a destination table whereas the attribute table produced in step 5 was designated as a source table. The two tables were combined by performing "Spatial Join", which performed the combination on the basis of the spatial relationship between the points in both the destination and source tables. Afterwards, the created new dataset was converted to a point-shape file, which visually displayed the vehicle trajectory along the test route.

Following the above six steps, the database was produced containing the driving measurements associated with each point along the route centerline, tagged with the time for each record. As mentioned previously, the database can be easily exported to various data formats for post processing and further analysis. It should be noted that the raw data of four drivers were missed in the VIU records. Thus only datasets for 26 drivers are available. The procedure of database construction is shown in Figure 4-1 and an example of the produced database is exhibited in Figure 4-2. 


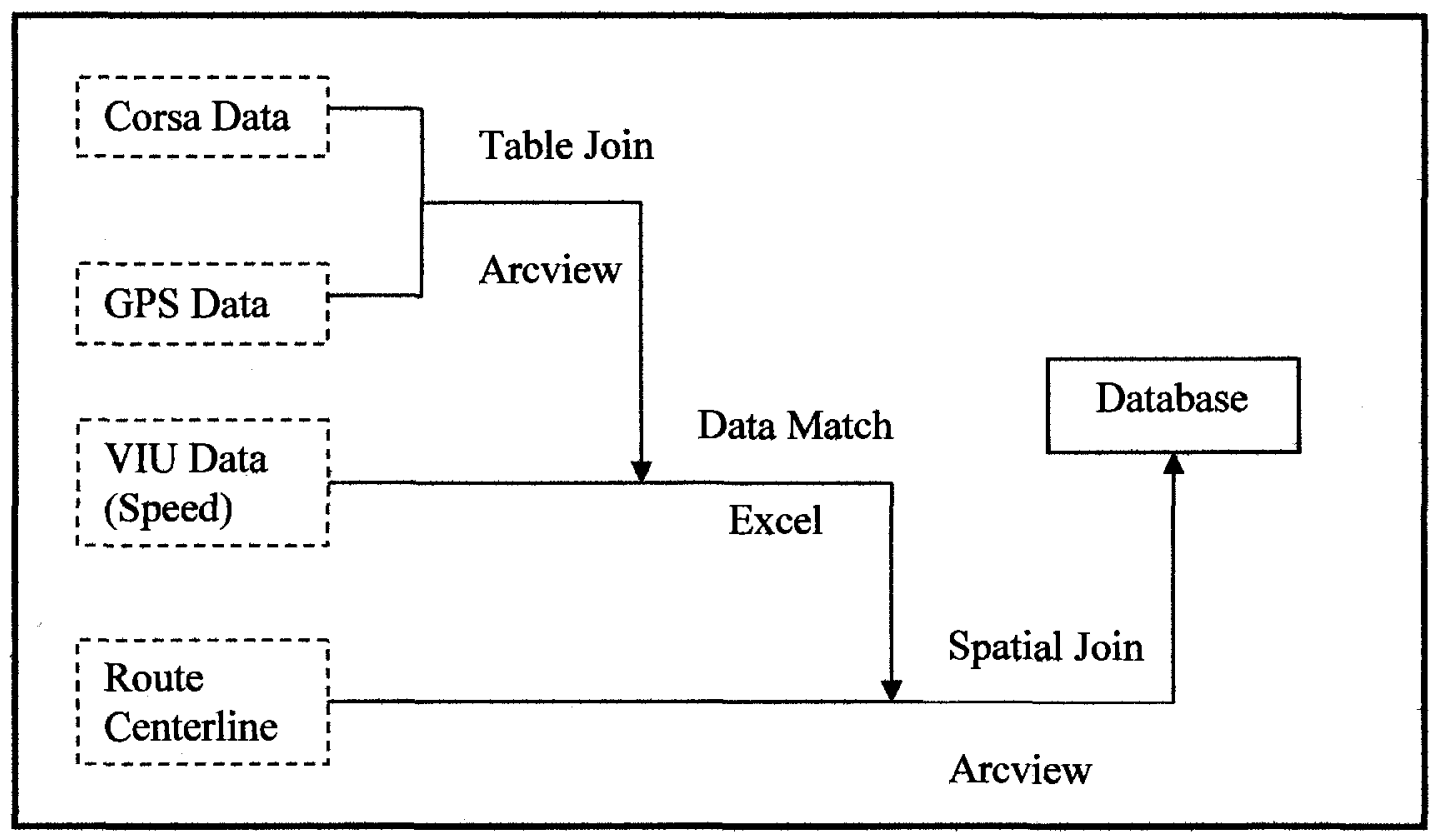

Figure 4-1: Procedure for Database Construction.

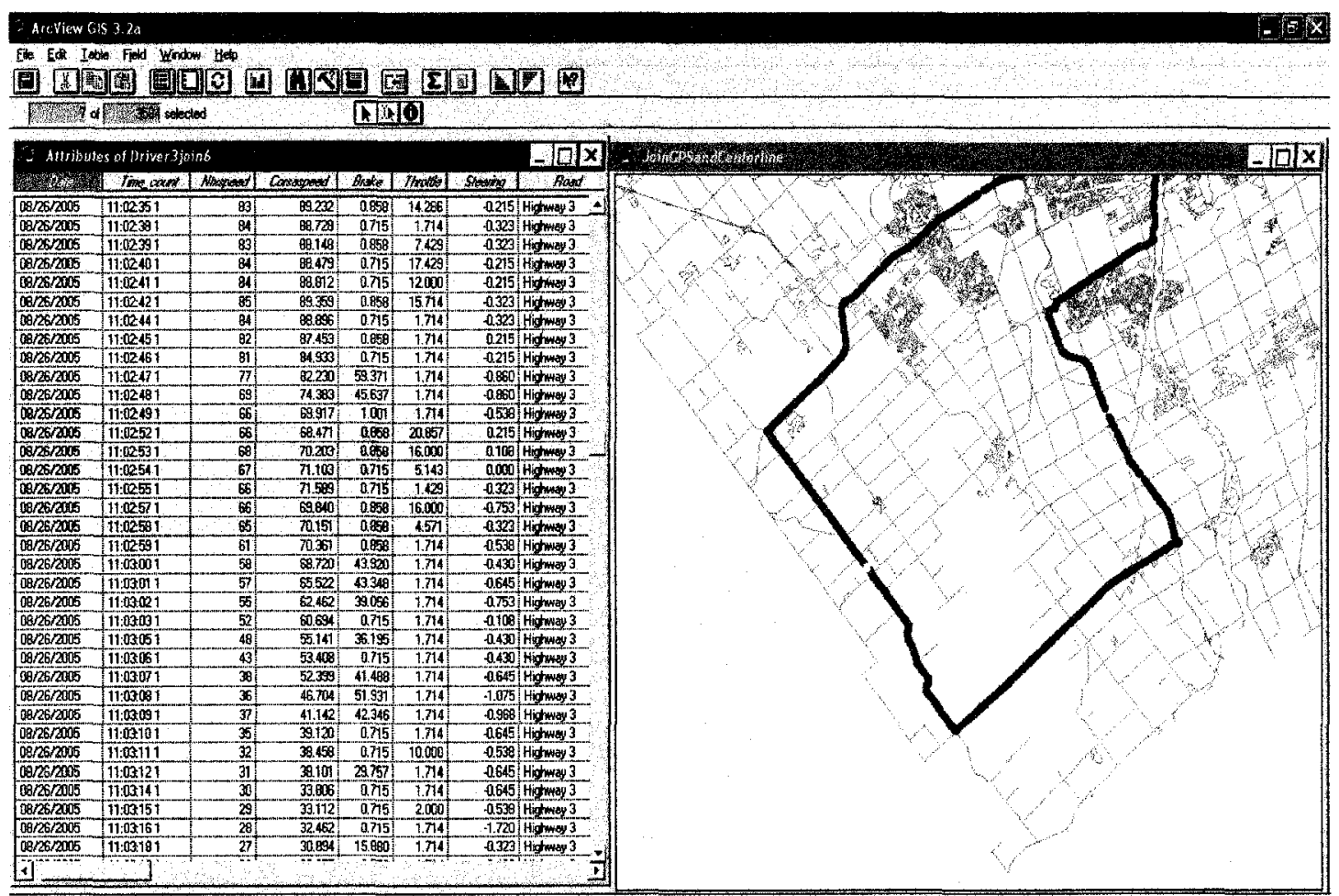

Figure 4-2: Example of Built Database. 


\subsection{Speed Comparison}

As mentioned earlier, each driver's instantaneous speeds were measured using two pieces of equipment, the Corsa Box and the VIU, as they drove on the route. In order to understand better the measurements of the two systems, the speeds recorded by the two systems were compared for each of the 26 drivers. The dataset for each driver was extracted to a delimited text format from the database built in Arcview, as explained earlier. In the following subsections, data reduction is explained. The results of the speed data comparison are then exhibited, followed by correlation analyses for the variables of speed difference, the Corsa speed, and the VIU speed.

\subsubsection{Speed Data Reduction}

A total of 88,159 pairs of speed data was recorded by both the Corsa and the VIU in the 26 trips. Some Corsa speed readings were screened out because of obvious discrepancies according to one of the following three conditions:

- The speed reading was apparently out of the range within which the driver would drive during the test, e.g., speed greater than $200 \mathrm{~km} / \mathrm{h}$.

- The single speed reading was not compatible with its adjacent speed readings. For example, speeds at the preceding second and following second were all less than $25 \mathrm{~km} / \mathrm{h}$, whereas only this single speed in-between was greater than $100 \mathrm{~km} / \mathrm{h}$.

- The abnormal speed readings that happened immediately after the Corsa system restarted from power failure during the test. 
In total, 300 Corsa speed readings were screened out, which accounted only for 0.34 percent of the available data. Thus, exclusion of these data from speed comparison and correlation analysis was not expected to affect the outcome.

\subsubsection{Difference between VIU and Corsa Speeds}

After screening out the obvious discrepancies of the speed readings, the differences between VIU speed and Corsa speed measurements were calculated in the Excel environment. An example of speed difference is shown in Figure 4-3. The summary of speed difference statistics for each driver is exhibited in Table 4-1. According to the outputs in Table 4-1, the speed observations by the VIU were generally 3 to $4 \mathrm{~km} / \mathrm{h}$ lower than those by the Corsa Box.

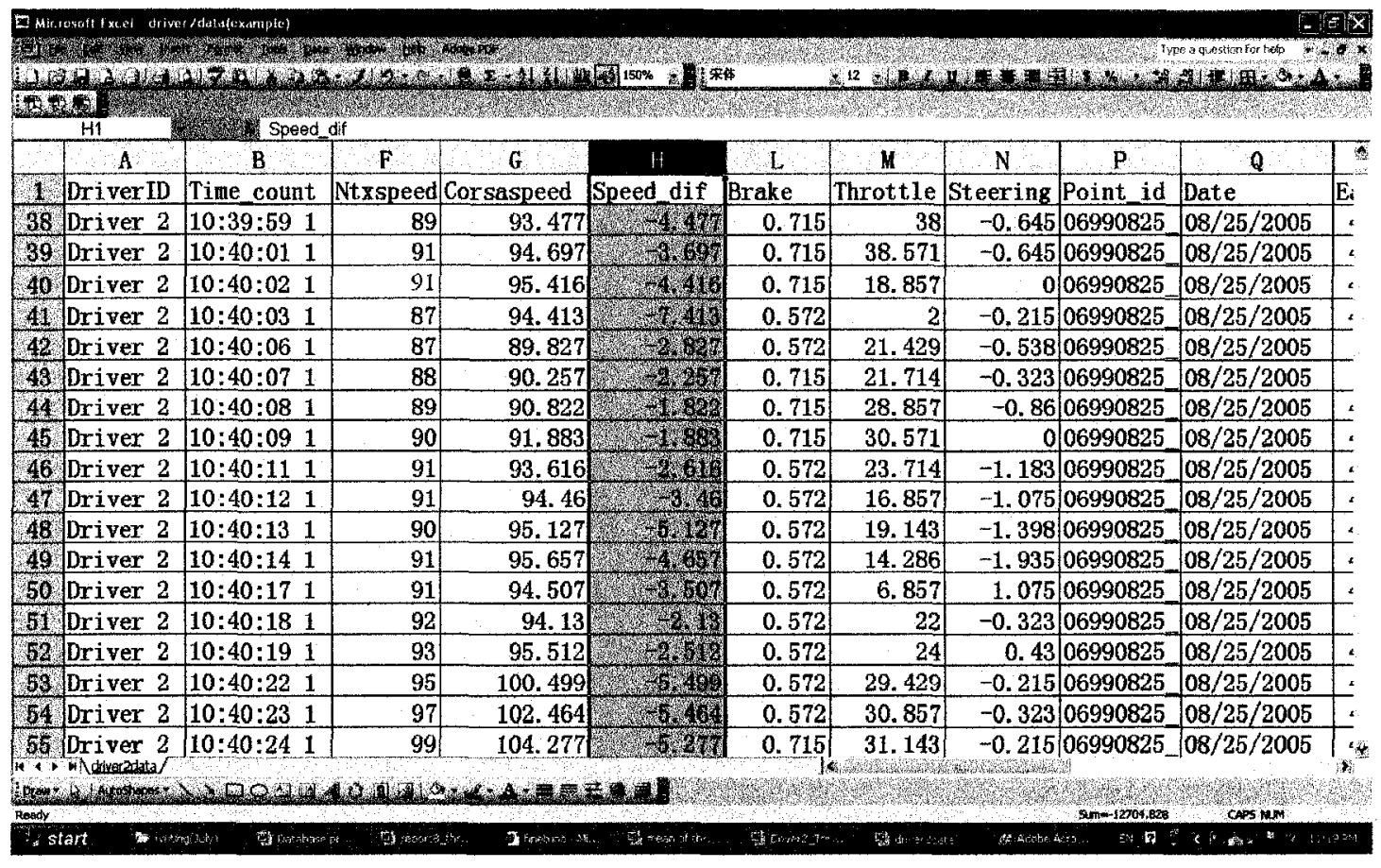

Figure 4-3: Example of Speed Difference. 
Table 4-1: The Speed Difference between the Two Systems (All Speed Pairs Included).

\begin{tabular}{|c|c|c|c|c|}
\hline Driver ID & $\mathbf{N}$ & Mean & Median & STD \\
\hline 02 & 3,194 & -4.166 & -4.460 & 2.739 \\
\hline 03 & 3,582 & -3.916 & -4.237 & 2.363 \\
\hline 04 & 3,264 & -3.636 & -4.110 & 3.875 \\
\hline 05 & 3,643 & -3.797 & -3.945 & 2.240 \\
\hline 06 & 3,066 & -3.693 & -4.128 & 2.651 \\
\hline 08 & 3,349 & -3.593 & -4.084 & 2.693 \\
\hline 09 & 3,212 & -3.886 & -4.420 & 2.626 \\
\hline 10 & 3,298 & -3.882 & -4.120 & 2.242 \\
\hline 11 & 3,235 & -3.802 & -4.118 & 2.229 \\
\hline 12 & 3,280 & -4.039 & -4.935 & 3.822 \\
\hline 13 & 3,243 & -4.202 & -4.500 & 2.654 \\
\hline 14 & 3,552 & -4.059 & -4.245 & 2.170 \\
\hline 15 & 2,984 & -3.977 & -4.480 & 2.653 \\
\hline 16 & 3,601 & -4.084 & -4.474 & 2.587 \\
\hline 17 & 3,533 & -3.533 & -3.838 & 3.017 \\
\hline 18 & 3,501 & -4.031 & -4.485 & 3.080 \\
\hline 19 & 3,715 & -3.605 & -3.928 & 2.171 \\
\hline 20 & 3,375 & -3.938 & -4.395 & 2.300 \\
\hline 21 & 3,553 & -3.871 & -4.189 & 2.138 \\
\hline 22 & 3,504 & -3.903 & -4.090 & 2.228 \\
\hline 23 & 3,767 & -3.581 & -3.949 & 2.688 \\
\hline 24 & 3,232 & -3.476 & -3.923 & 2.927 \\
\hline 25 & 4,069 & -3.760 & -3.966 & 2.379 \\
\hline 26 & 2,967 & -3.920 & -4.249 & 2.317 \\
\hline 31 & 3,186 & -3.918 & -4.350 & 2.858 \\
\hline 32 & 2,954 & -4.173 & -4.469 & 2.666 \\
\hline Total & 87,859 & -3.858 & -4.211 & 2.663 \\
\hline
\end{tabular}

Note 1: Speed difference $=$ VIU speed - Corsa speed

Note 2: Speed is in units of $\mathrm{km} / \mathrm{h}$.

STD: Standard deviation. 
Table 4-2: The Speed Difference between the Two Systems (Excluded Speed Pairs if Corsa Speeds Were Less than $20 \mathrm{~km} / \mathrm{h}$ ).

\begin{tabular}{|c|c|c|c|c|}
\hline Driver ID & $\mathbf{N}$ & Mean & Median & STD \\
\hline 02 & 3,112 & -4.358 & -4.490 & 2.187 \\
\hline 03 & 3,464 & -4.151 & -4.272 & 1.624 \\
\hline 04 & 3,163 & -3.916 & -4.149 & 3.099 \\
\hline 05 & 3,544 & -3.952 & -3.980 & 1.804 \\
\hline 06 & 2,920 & -3.996 & -4.195 & 1.932 \\
\hline 08 & 3,193 & -3.909 & -4.125 & 1.808 \\
\hline 09 & 3,082 & -4.193 & -4.481 & 1.783 \\
\hline 10 & 3,199 & -4.081 & -4.156 & 1.696 \\
\hline 11 & 3,151 & -3.985 & -4.148 & 1.687 \\
\hline 12 & 3,161 & -4.373 & -5.005 & 3.205 \\
\hline 13 & 3,150 & -4.378 & -4.552 & 2.259 \\
\hline 14 & 3,454 & -4.203 & -4.292 & 1.702 \\
\hline 15 & 2,879 & -4.239 & -4.517 & 1.933 \\
\hline 16 & 3,483 & -4.345 & -4.523 & 1.803 \\
\hline 17 & 3,445 & -3.715 & -3.864 & 2.467 \\
\hline 18 & 3,395 & -4.255 & -4.532 & 2.450 \\
\hline 19 & 3,573 & -3.839 & -3.983 & 1.502 \\
\hline 20 & 3,277 & -4.163 & -4.440 & 1.654 \\
\hline 21 & 3,436 & -4.092 & -4.227 & 1.429 \\
\hline 22 & 3,407 & -4.076 & -4.129 & 1.725 \\
\hline 23 & 3,633 & -3.823 & -3.981 & 1.928 \\
\hline 24 & 3,027 & -3.867 & -3.994 & 2.061 \\
\hline 25 & 3,965 & -3.928 & -3.995 & 1.913 \\
\hline 26 & 2,876 & -4.147 & -4.302 & 1.705 \\
\hline 31 & 3,077 & -4.164 & -4.386 & 2.069 \\
\hline 32 & 2,875 & -4.370 & -4.499 & 2.095 \\
\hline Total & 84,941 & -4.091 & -4.251 & 2.028 \\
\hline
\end{tabular}

Note 1: Speed difference $=$ VIU speed - Corsa speed

Note 2: Speed is in units of $\mathrm{km} / \mathrm{h}$.

STD: Standard deviation. 
Another speed difference analysis was undertaken for the speed pairs if the Corsa speeds were equal to or greater than $20 \mathrm{~km} / \mathrm{h}$ (Table 4-2). This analysis was performed due to the observation that Corsa speed readings at low speeds sometimes were discrepant from the supposed actual speeds to a large extent. For example, when the vehicle approached a stop sign, speeds less than $15 \mathrm{~km} / \mathrm{h}$ were recorded as zero. Compared with the results in Table 4-1, the mean speed difference increases and the standard deviation decreases for all individual drivers when low speeds were excluded from the computation. These results were expected since more positive speed differences exist at low speeds.

\subsubsection{Correlation Analysis of Speed Data}

In order to investigate the correlations among the three variables: speed difference, VIU speed, and Corsa speed, correlation analysis was conducted using Bivariate Correlations in the software Statistical Package for Social Science (SPSS v15). The Bivariate Correlations tool in SPSS v15 is capable of qualifying the degree of covariation among sets of variables with three fundamental dimensions: significance, direction, and magnitude. Correlation analyses were performed for two conditions:

- All speed measurements and speed differences were used in the analysis (Table 4-3).

- The data (Corsa speeds, VIU speeds, and their speed differences) were sorted into two groups by the Corsa speeds: speeds less than $20 \mathrm{~km} / \mathrm{h}$, and speeds equal to or greater than $20 \mathrm{~km} / \mathrm{h}$. Analyses were conducted for each of the two groups (Table 4-4 and 4-5). 
Table 4-3: Results of Correlation Analysis (All Speed Data Included).

\begin{tabular}{|l|l|r|r|r|}
\hline \multirow{5}{*}{ VIU Speed } & $\begin{array}{l}\text { VIU } \\
\text { Speed }\end{array}$ & $\begin{array}{l}\text { Corsa } \\
\text { Speed }\end{array}$ & $\begin{array}{l}\text { Speed } \\
\text { Difference }\end{array}$ \\
\hline \multirow{5}{*}{ Corsa Speed } & Pearson Correlation & 1 & $.989\left(^{* *}\right)$ & $-.475\left(^{* *}\right)$ \\
\cline { 2 - 5 } & Sig. (2-tailed) &. & .000 & .000 \\
\cline { 2 - 5 } & $\mathrm{N}$ & 88,159 & 88,159 & 87,859 \\
\hline \multirow{3}{*}{ Speed Difference } & Pearson Correlation & $.989\left(^{* *}\right)$ & 1 & $-.549\left(^{* *}\right)$ \\
\cline { 2 - 5 } & Sig. (2-tailed) & .000 & .000 \\
\cline { 2 - 5 } & $\mathrm{N}$ & 88,159 & 88,159 & 87,859 \\
\cline { 2 - 5 } & Pearson Correlation & $-.475\left(^{* *}\right)$ & $-.549\left(^{* *}\right)$ & 1 \\
\cline { 2 - 5 } & Sig. (2-tailed) & .000 & .000 & 87,859 \\
\cline { 2 - 5 } & $\mathrm{N}$ & 87,859 & 87,859 &. \\
\hline
\end{tabular}

** Correlation is significant at the 0.01 level of significance (2-tailed).

Table 4-4: Results of Correlation Analysis (Corsa Speeds Less than $20 \mathrm{~km} / \mathrm{h}$ ).

\begin{tabular}{|l|l|r|r|r|}
\hline \multirow{5}{*}{ VIU Speed } & $\begin{array}{l}\text { VIU } \\
\text { Speed }\end{array}$ & $\begin{array}{l}\text { Corsa } \\
\text { Speed }\end{array}$ & $\begin{array}{l}\text { Speed } \\
\text { Difference }\end{array}$ \\
\hline \multirow{5}{*}{ Corsa Speed } & Pearson Correlation & 1 & $.416\left(^{* *}\right)$ & $.427\left(^{* *}\right)$ \\
\cline { 2 - 5 } & Sig. (2-tailed) &. & .000 & .000 \\
\cline { 2 - 5 } & $\mathrm{N}$ & 2985 & 2985 & 2918 \\
\hline \multirow{3}{*}{ Speed Difference } & Pearson Correlation & $.416\left(^{* *}\right)$ & 1 & $-.506\left(^{* *}\right)$ \\
\cline { 2 - 5 } & Sig. (2-tailed) & .000 & .000 \\
\cline { 2 - 5 } & $\mathrm{N}$ & 2985 & 2985 & 2918 \\
\cline { 2 - 5 } & Pearson Correlation & $.427(* *)$ & $-.506\left(^{* *}\right)$ & 1 \\
\cline { 2 - 5 } & Sig. (2-tailed) & .000 & .000 & 2918 \\
\cline { 2 - 5 } & $\mathrm{N}$ & 2918 & 2918 & .000 \\
\hline
\end{tabular}

** Correlation is significant at the 0.01 level of significance (2-tailed). 
Table 4-5: Results of Correlation Analysis (Corsa Speeds Equal to or Greater than 20 $\mathrm{km} / \mathrm{h})$.

\begin{tabular}{|l|l|l|l|l|}
\hline \multirow{5}{*}{ VIU Speed } & & $\begin{array}{l}\text { VIU } \\
\text { Speed }\end{array}$ & $\begin{array}{l}\text { Corsa } \\
\text { Speed }\end{array}$ & $\begin{array}{l}\text { Speed } \\
\text { Difference }\end{array}$ \\
\hline \multirow{5}{*}{ Corsa Speed } & Pearson Correlation & 1 & $.988\left(^{* *}\right)$ & $-.384\left(^{* *}\right)$ \\
\cline { 2 - 5 } & Sig. (2-tailed) &. & .000 & .000 \\
\cline { 2 - 5 } & $\mathrm{N}$ & 85174 & 85174 & 84941 \\
\hline \multirow{3}{*}{ Speed Difference } & Pearson Correlation & $.988\left(^{* *}\right)$ & 1 & $-.457\left(^{* *}\right)$ \\
\cline { 2 - 5 } & Sig. (2-tailed) & .000 &. & .000 \\
\cline { 2 - 5 } & $\mathrm{N}$ & 85174 & 85174 & 84941 \\
\cline { 2 - 5 } & Pearson Correlation & $-.384\left(^{* *}\right)$ & $-.457(* *)$ & 1 \\
\cline { 2 - 5 } & Sig. (2-tailed) & .000 & .000 &. \\
\cline { 2 - 5 } & $\mathrm{N}$ & 84941 & 84941 & 84941 \\
\hline
\end{tabular}

** Correlation is significant at the 0.01 level of significance (2-tailed).

Referring to the outcomes of correlation analysis for the first condition (Table 4-3), the speed difference has a moderate correlation with both the VIU speed and Corsa speed, and the correlation is statistically significant at the 0.01 level of significance in the twotailed test. The sign of the correlation coefficient indicates that the speed difference decreases as the actual speed increases. Since the mean speed difference is negative, the interpretation of these negative correlations is that the speed measurements of the VIU are generally less than the measurements of the Corsa system especially as the vehicle's actual speed becomes higher.

Referring to the outcomes of correlation analysis for the group of Corsa speeds less than $20 \mathrm{~km} / \mathrm{h}$ (Table 4-4), the speed difference has a negative moderate correlation with the Corsa speed and a positive moderate correlation with the VIU speed, and both correlations are statistically significant at the 0.01 level of significance in the two-tailed test. The interpretation of these correlations is that, under low-speed conditions, the speed 
difference generally decreases with the increase of the Corsa speed measurement while it increases with the increase of the VIU speed measurement. As previously mentioned, it is found that the Corsa Box sometimes recorded low speeds as zero. Therefore, it is understandable that the speed difference increases with the increase of the VIU speed. In addition, the correlation between the VIU speed and the Corsa speed in Table 4-4 is much smaller if compared with those in Table 4-3 and 4-5. It indicates that the changes of these two speed measurements are in a relatively lower accordance in the low speed range. All this information may imply that the Corsa Box is less sensitive in measuring low speeds than the VIU.

In the group of Corsa speeds equal to or greater than $20 \mathrm{~km} / \mathrm{h}$ (Table $4-5)$, there were 84,941 records of speed differences between the VIU speeds and the Corsa speeds. Referring to the outcomes of the correlation analysis, the speed difference has a negative moderate correlation with both the VIU speed and the Corsa speed, and both correlations are statistically significant at the 0.01 level of significance in the two-tailed test. These negative correlations indicate that the speed difference between the two speed measures becomes bigger with the increase of vehicular speed, and the VIU in general recorded lower speeds than the Corsa did, in the range of the Corsa speed equal to or greater than $20 \mathrm{~km} / \mathrm{h}$. This result is in accordance with the previous analysis that contains all speeds.

\subsubsection{Summary of Speed Comparison}

Based on the above analysis, one could reach the following findings with respect to the comparison of speed measurements between the VIU and the Corsa system: 
- In general, the speed measurements of the Corsa system are higher than those of the VIU.

- The measure of the Corsa system may exhibit low sensitivity for low speed conditions (i.e., less than $20 \mathrm{~km} / \mathrm{h}$ ).

- The speed difference between these two measurements becomes greater with the increase of the actual driving speed when the driving speed is higher than 20 $\mathrm{km} / \mathrm{h}$.

- The Corsa speeds show higher correlations with the speed differences than the VIU speeds, which implies that the Corsa speed accounts more for the change of speed differences.

\subsection{Throttle Data Comparison}

The measurements of throttle use represent the throttle position during driving in units of percent. In theory, a value of 100 means the maximum throttle use when the throttle pedal is fully pressed and 0 means the minimum status of throttle use when the throttle pedal is not touched. Since both the Corsa Box and the VIU were employed in detecting throttle use during the test, the measurements of the two systems are compared in this section. First, the procedure of combining the Corsa and VIU throttle data is briefly presented, then a comparison of the raw throttle data of the two systems is discussed, and finally a comparison of the calibrated throttle data of the two systems is explained.

Because raw VIU throttle data were provided in a separate file from VIU speed data, an extra effort was made to build another database for the throttle data that contains both 
the VIU and Corsa throttle data. The procedure for building the throttle database was similar to that for building the speed database:

- The Corsa throttle data were extracted from the database, which was built after the completion of step 2 in Section 4.1.2.

- The VIU throttle data for each driver were sorted and separated into individual files according to their experiment time.

- For each individual driver, the Corsa throttle data were filtered to match the VIU data according to their common time stamp.

A total of 17,994 pairs of throttle data was recorded by both the Corsa Box and the VIU in the 26 trips. An example of combined throttle data is exhibited in Figure 4-4.

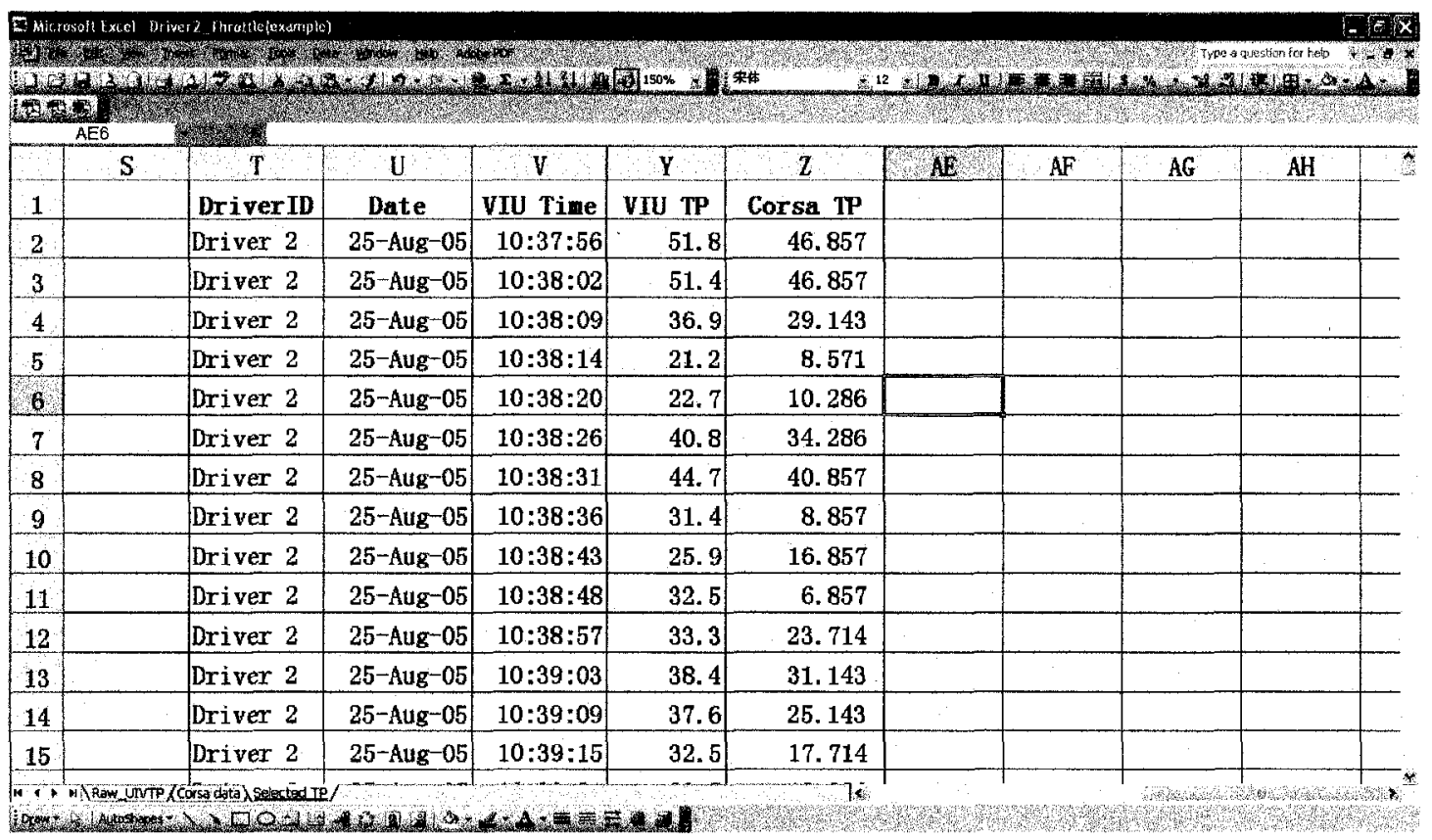

Figure 4-4: Example of Combined Throttle Data. 


\subsubsection{Difference of Raw Throttle data}

The differences of throttle data between the two systems were computed using the raw VIU measurement minus raw Corsa measurement. The summary of the throttle difference statistics for each driver is presented in Table 4-6. The results show that, in general, the VIU detected more throttle use than the Corsa Box did. However, the minimum throttle usages recorded by the two systems were substantially different, as shown in Table 4-7. Therefore, these outcomes may be biased due to their distinctive origins. For a meaningful comparison between the two systems, the raw throttle observations needed to be calibrated. 
Table 4-6: The Difference of Throttle Data Using the Raw data.

\begin{tabular}{|c|c|c|c|}
\hline Driver ID & Mean & Median & STD \\
\hline 02 & 10.516 & 10.071 & 4.377 \\
\hline 03 & 11.833 & 10.771 & 5.662 \\
\hline 04 & 12.141 & 11.029 & 5.357 \\
\hline 05 & 10.813 & 10.150 & 5.738 \\
\hline 06 & 11.057 & 10.871 & 3.192 \\
\hline 08 & 11.127 & 10.529 & 3.522 \\
\hline 09 & 10.516 & 9.686 & 3.430 \\
\hline 10 & 11.455 & 11.157 & 3.403 \\
\hline 11 & 11.127 & 10.857 & 3.614 \\
\hline 12 & 10.520 & 9.136 & 6.175 \\
\hline 13 & 10.241 & 9.486 & 5.757 \\
\hline 14 & 11.277 & 10.486 & 4.417 \\
\hline 15 & 10.645 & 10.071 & 4.528 \\
\hline 16 & 11.307 & 10.257 & 5.885 \\
\hline 17 & 12.418 & 11.443 & 5.681 \\
\hline 18 & 10.800 & 9.186 & 5.701 \\
\hline 19 & 11.823 & 11.271 & 3.144 \\
\hline 20 & 10.900 & 10.257 & 4.307 \\
\hline 21 & 11.636 & 10.700 & 4.137 \\
\hline 22 & 11.178 & 10.108 & 6.809 \\
\hline 23 & 11.457 & 10.943 & 3.970 \\
\hline 24 & 11.726 & 11.500 & 3.380 \\
\hline 25 & 11.827 & 11.786 & 6.279 \\
\hline 26 & 10.574 & 10.079 & 3.984 \\
\hline 31 & 10.671 & 9.971 & 3.732 \\
\hline 32 & 10.682 & 9.729 & 3.823 \\
\hline Total & 11.190 & 10.586 & 4.768 \\
\hline
\end{tabular}

Note: Throttle difference $=$ VIU throttle - Corsa throttle STD: Standard deviation. 
Table 4-7: Minimum and Maximum Throttle Usages Recorded by the VIU and Corsa.

\begin{tabular}{|c|c|c|}
\hline & VIU Measurement & Corsa Measurement \\
\hline Minimum & 19.6 & 0.857 \\
\hline Maximum & 76.1 & 68.857 \\
\hline Range & 56.5 & 68 \\
\hline
\end{tabular}

\subsubsection{Difference of Calibrated Throttle data}

The objective of calibration was to convert the raw readings of throttle position to new readings on a scale from zero to 100 . Equation 4.1 was used for the calibration.

$$
T P_{n e w}=\frac{t p_{i}-t p_{\min }}{t p_{\max }-t p_{\min }} \times 100
$$

Where $T P_{n e w}$ is the converted throttle position measurement, $t p_{i}$ is the raw throttle position measurement, $t p_{\min }$ is the minimum value of the raw throttle position measurement, and $t p_{\max }$ is the maximum value of the raw throttle position measurement.

After calibration of the raw throttle data, throttle data comparison was conducted using the calibrated data. The summary statistics of the difference of calibrated throttle data are exhibited in Table 4-7. Similar to the comparison using raw throttle data, the differences here are equal to the calibrated VIU data minus the calibrated Corsa data. 
Table 4-8: The Difference of Throttle Data Using the Calibrated data.

\begin{tabular}{|c|c|c|c|}
\hline Driver ID & Mean & Median & STD \\
\hline 02 & -9.784 & -10.589 & 6.100 \\
\hline 03 & -7.906 & -9.149 & 8.120 \\
\hline 04 & -8.736 & -9.426 & 5.673 \\
\hline 05 & -9.181 & -9.943 & 8.303 \\
\hline 06 & -9.269 & -9.593 & 4.390 \\
\hline 08 & -9.110 & -9.967 & 4.869 \\
\hline 09 & -9.601 & -10.411 & 4.446 \\
\hline 10 & -8.648 & -9.041 & 4.545 \\
\hline 11 & -8.950 & -9.462 & 4.955 \\
\hline 12 & -8.363 & -9.661 & 8.496 \\
\hline 13 & -8.887 & -10.075 & 7.686 \\
\hline 14 & -8.616 & -9.571 & 5.878 \\
\hline 15 & -9.557 & -10.363 & 6.507 \\
\hline 16 & -8.549 & -9.608 & 6.910 \\
\hline 17 & -8.232 & -9.017 & 7.148 \\
\hline 18 & -8.187 & -10.192 & 7.259 \\
\hline 19 & -8.201 & -8.863 & 4.070 \\
\hline 20 & -8.487 & -9.667 & 5.335 \\
\hline 21 & -8.078 & -9.282 & 5.412 \\
\hline 22 & -8.599 & -10.218 & 10.017 \\
\hline 23 & -8.859 & -9.593 & 5.645 \\
\hline 24 & -8.529 & -8.908 & 4.917 \\
\hline 25 & -7.846 & -8.308 & 9.028 \\
\hline 26 & -9.265 & -10.064 & 5.306 \\
\hline 31 & -9.551 & -10.589 & 5.089 \\
\hline 32 & -9.024 & -10.254 & 4.758 \\
\hline Total & -8.765 & -9.593 & 6.395 \\
\hline
\end{tabular}

Note: Throttle difference $=$ VIU throttle - Corsa throttle STD: Standard deviation. 
This comparison is more reasonable because the distinction between the minimum observations of the two systems was treated. According to the outcomes in Table 4-8, on average the throttle usages recorded by the VIU were less than those by the Corsa. In summary, the measurements of the two systems in detecting throttle position show general discrepancies. The disparity of the two systems might be attributed to their different detection modes: the VIU operated through the port of on-board diagnostics (OBDII), which is inherent in the vehicle, whereas the Corsa system detected throttle position through the sensor attached on the throttle itself. 


\section{CHAPTER 5: DRIVER SPEED BEHAVIOUR}

This chapter presents the analysis of driver speed behaviour corresponding to each road type under investigation. In addition, one interest of this research is to identify the relative driving manner of individual drivers in terms of aggressive, common, and defensive. Therefore, this chapter also covers the procedure for distinguishing three categories of drivers in the driver sample and the comparison of speed choices of the three driver categories. Before analyzing driver speed behaviour, the database was refined. This mainly involved the identification of free-flow speeds and non-free-flow speeds.

Free-flow speed indicates that a vehicle's speed is believed to be free from the constraints of outside traffic, whereas non-free-flow speed denotes that a vehicle's speed is believed to be under the constraints of other vehicles. Identification of free-flow and non-free-flow speeds is an essential step for studying driver speed behaviour, because driver speed choice under these two conditions can vary substantially. For example, drivers may have to slow down their vehicles if there is a slow moving vehicle in front impeding traffic and the driver has no chance to pass or change lanes. On the other hand, if there is no close vehicle ahead of them, drivers are likely to speed up and travel at their desired speed. Time headway, defined as the time elapsed for two successive vehicles passing the same point, is popularly used to identify free-flow and non-free-flow speeds. Generally, time headway can be computed by dividing the distance between two successive vehicles, as measured by the front laser gun, by the instantaneous speed of the trailing vehicle. 


\subsection{Database Refinement}

The objective of database refinement was to make the data applicable for statistical analysis using a statistics package such as SPSS v15.0. According to the findings in the foregoing chapter, the Corsa Box may present low sensitivity for low speeds. Thus the VIU speeds were used for analysis in this chapter, and they were also used to compute the acceleration rates in the next chapter.

An example of the refined database in Excel for Driver 6 is given in Figure 5-1.

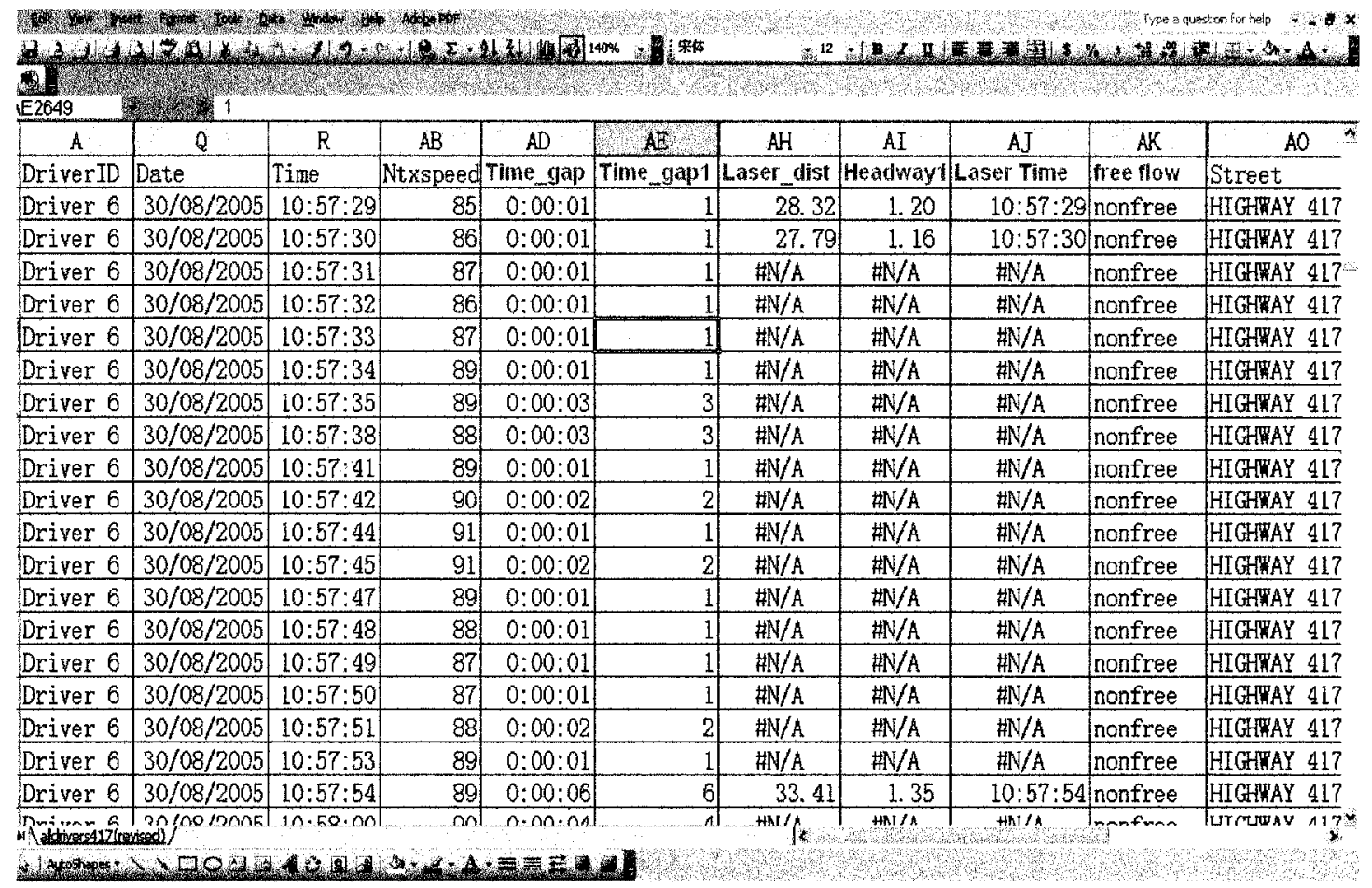

Figure 5-1: Example of Refined Database in Excel.

Database refinement involved the following steps:

1. The datasets for individual drivers were exported from Arcview 3.2a to delimited text files. Since the database built in Arcview 3.2a set up a link between driver operational data and the road centerline along the test route, the instantaneous 
speeds can be determined in relation to specific road type. As shown in the example of Figure 5-1, these speeds for Driver 6 took place on Highway 417.

2. As mentioned earlier, the VIU did not record speeds at every second if the speed remained constant. Thus the duration of each driving speed was computed on the basis of the time difference between this record and the successive record. Column $\mathrm{AD}$ in the example presents the computed time difference. This time difference was then converted from time format to numeric format, which denoted the speed duration in units of seconds as shown in Column AE in Figure 5-1. The time difference in numeric format was used for calculating speed frequency and computing acceleration rate.

3. The front laser gun data, which contain the measured distances between the test vehicle and the vehicle preceding, were matched in Excel with corresponding speeds with the same time reference. In this example, Column $\mathrm{AH}$ shows the distances measured with the laser gun, in which "\#N/A" denotes that no distance data is available. The physical meaning of this condition is explained later in this section.

4. Time headway was computed for the records, whenever the laser gun distance data was available, by dividing the measured distance over the observed speed. In this example, Column AI shows the calculated time headway in units of seconds. As in previous research, a headway of 5-second was applied to distinguish freeflow speeds from non-free-flow speeds for freeways and two-lane rural highways. Nevertheless, on urban and suburban roads, high traffic volume is expected and intersections appear more frequently. Furthermore, driving speeds can be affected 
by other factors such as on-street parking and pedestrian activity in the vicinity of the roadway. Therefore, a 5-second headway is seldom expected on urban and suburban roads. Rather, a 3-second headway is used in this research for separating free-flow and non-free-flow speeds on urban/suburban roads as recommended by Pasanen and Salmivaara (1993). If the time headway was greater than the threshold of 5 seconds (or 3 seconds for urban/suburban roads), this speed was sorted as free-flow speed; otherwise it was non-free-flow speed.

5. Although distance measured using laser guns is an effective parameter that can be directly used to recognize free-flow and non-free-flow speeds, one concern is judging the speeds whose corresponding laser gun distance was unavailable. Thus, video was employed to assist in judging those speeds whose corresponding laser gun distance was unavailable. There are three cases.

- In one case, the same leading vehicle stayed in front for the period where the distance measurement was not available, and the computed headways at the two ends of this period were either larger or lower than the headway threshold. The speeds in this case were judged as free-flow or non-free-flow depending on whether the headways at the two ends were greater or lower than the headway threshold, respectively.

- In the second case, although the same leading vehicle stayed in front, speeds of the test vehicle at the ends of the period where no distance measurements were available changed from free-flow to non-free-flow conditions, and vice versa. The interpolation method was applied in this case to compute the distances for those speeds in between, using the distances at the two ends and 
assuming a linear rate of distance change. Time headways were then computed using the interpolated distances and their corresponding speeds. Thereafter, the speeds in between were distinguished as free-flow or non-freeflow, according to the computed headways.

- In another case, measured distances in a certain period were associated with different leading vehicles due to lane-change by the test vehicle or the leading vehicles. The lane-change moments were marked according to the video, therefore, this case can be transformed into and treated as one of the two cases stated above.

6. Following the abovementioned criteria, each individual speed was marked as free-flow speed or non-free-flow speed, using a newly introduced column in Excel. As shown in the example, Column AK indicates that the speeds during the time period from 10:57:29 a.m. to 10:57:54 a.m. for Driver 6 were all under nonfree-flow conditions on Highway 417.

7. After completion of the above steps in Excel, the data were loaded into SPSS and a dummy variable was introduced to denote free-flow or non-free-flow conditions.

8. Since each line of records has been marked with a specific road, another dummy variable was introduced in the SPSS file to describe the four road classes: urban freeway, rural freeway, two-lane rural highway, and urban/suburban road. As stated earlier, Highway 417 is an urban freeway; Highway 416 is a rural freeway; Provincial Road 7, and Regional Roads 3 and 6 belong to two-lane rural highways; and Regional Roads 12 and 73 are urban/suburban roads. 
Following the above procedure, a refined database was established in SPSS v15.0 for further statistical analysis. The methodology and results relative to driver speed behaviour analysis are presented in the following section.

\subsection{Driver Speed Behaviour on Different Road Classes}

This section presents the results of driver speed behaviour analysis, which was performed aggregately for the driver sample on each of the four road types under the three speed conditions: free-flow condition, non-free-flow condition and mixed flow condition, which is a combination of free-flow and non-free-flow. For each road type, speed distribution was used to describe the speed frequency by speed ranges. Each speed range has an interval of $10 \mathrm{~km} / \mathrm{h}$, of which the lower boundary speed is inclusive whereas the upper boundary speed is exclusive. The speed duration was counted in seconds and its corresponding time percentage on this road type was computed as well. In addition, a histogram, in which the percentage of each speed range was computed for the corresponding flow conditions, was used to illustrate the speed distributions for the three flow conditions. The results of driver speed behaviour on different road classes are individually introduced in the following subsections.

\subsubsection{Urban Freeway (Highway 417)}

In addition to inter-city and inter-provincial traffic, Highway 417 serves a significant volume of east-west commuting traffic across the City of Ottawa. The posted speed limit (PSL) on Highway 417 is $100 \mathrm{~km} / \mathrm{h}$. 
The speed distribution for Highway 417 is summarized in Table 5-1 and the results are also illustrated in Figure 5-2. There was a total of 22,068 seconds of travel time on Highway 417 by the 26 drivers. The observed speeds ranged from 34 to $137 \mathrm{~km} / \mathrm{h}$, and the average speed for the driver sample was $101.23 \mathrm{~km} / \mathrm{h}$ for mixed flow conditions. This average speed was very close to the PSL of $100 \mathrm{~km} / \mathrm{h}$. However it was examined to be statistically different from the PSL at $5 \%$ level of significance, using the one-sample ttest in SPSS v15.0. Nearly 60 percent of the speeds were above or equal to the PSL, of which two-thirds fell in the interval of $10 \mathrm{~km} / \mathrm{h}$ above the PSL. In summary, most of the speeds for the driver sample were in the range between 90 and $120 \mathrm{~km} / \mathrm{h}$. The pace speed, which denotes a speed range with the most frequently observed speeds, was from 100 to $110 \mathrm{~km} / \mathrm{h}$.

By comparing the free-flow and non-free-flow speeds, around one quarter of the speeds took place under free-flow conditions whereas the other three quarters of speeds were under non-free-flow conditions on Highway 417, as shown in Table 5-1. The average speed under free-flow conditions was about $4 \mathrm{~km} / \mathrm{h}$ higher than that under nonfree-flow conditions. In order to examine if these two mean speeds were statistically different, a t-test of two independent samples was performed in SPSS v15.0. The output suggests that the two mean speeds were significantly different at $5 \%$ level of significance. In addition, both of the two mean speeds were also statistically different from the posted speed of 100, as checked in SPSS v15.0 using the one-sample t-test. In addition, the most evident difference of the speed distribution between free-flow and non-free-flow conditions appeared in two speed ranges: 90 to $100 \mathrm{~km} / \mathrm{h}$ and 110 to $120 \mathrm{~km} / \mathrm{h}$. A higher percentage of non-free-flow speeds occurred in the speed range between 90 to $100 \mathrm{~km} / \mathrm{h}$ than free-flow speeds, while a larger percentage of the free-flow speeds existed in the speed range between 110 to $120 \mathrm{~km} / \mathrm{h}$ than non-free-flow speeds. 


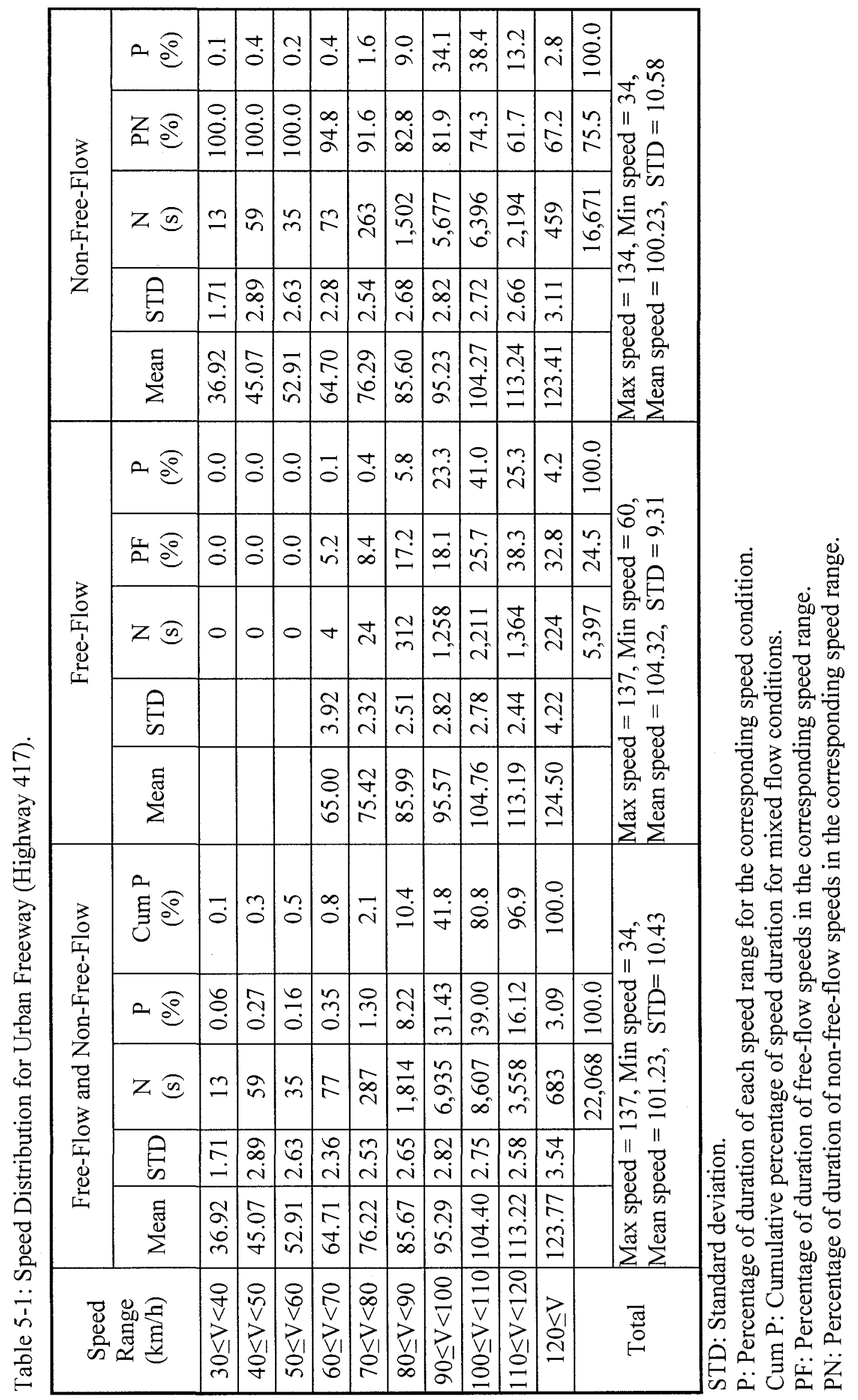




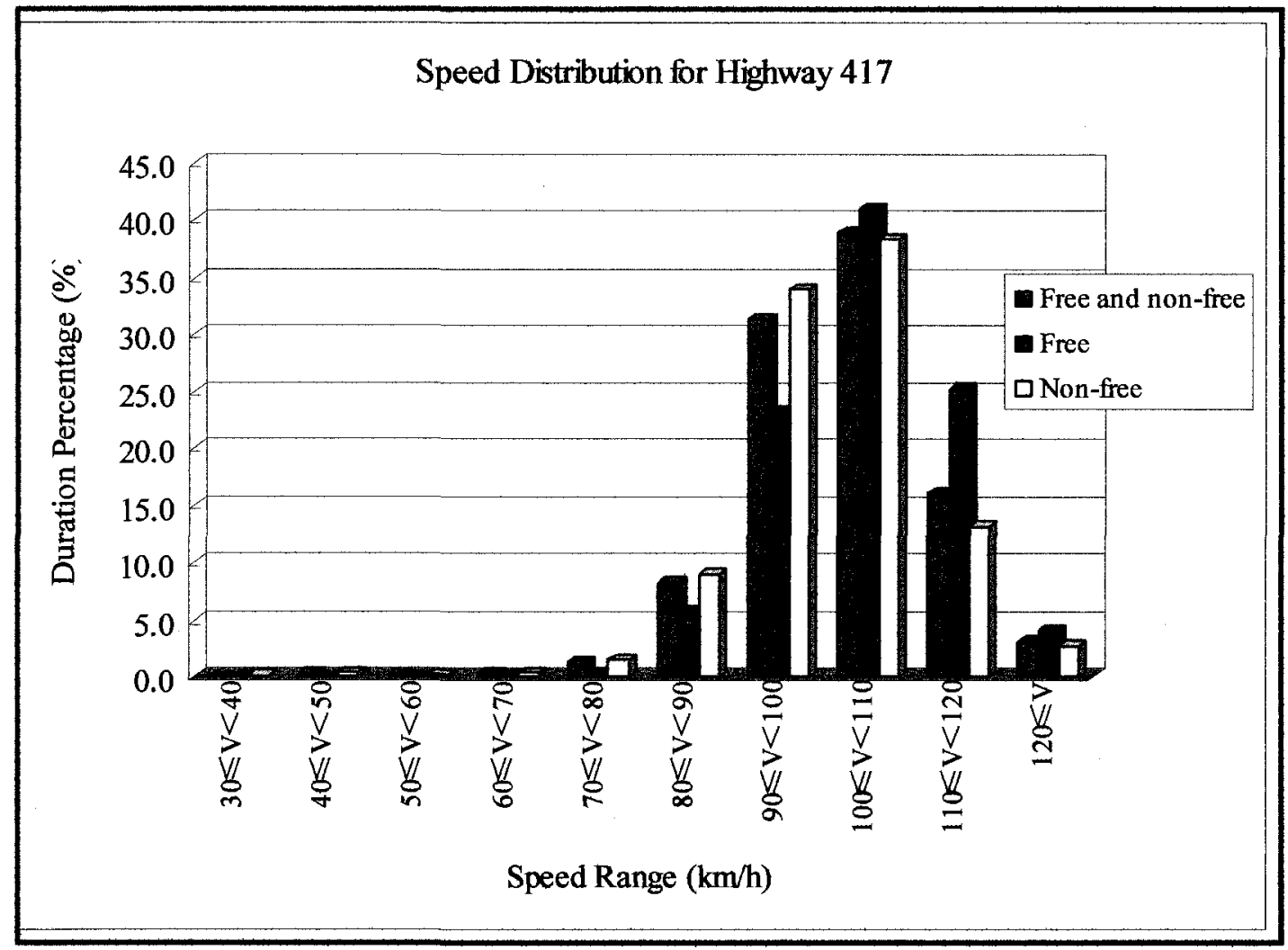

Figure 5-2: Speed Distribution for Urban Freeway (Highway 417).

\subsubsection{Two-Lane Rural Highways}

Provincial Route 7 and Regional Routes 3 and 6 on the selected test route are typical undivided two-lane rural highways on which the speed limit is $80 \mathrm{~km} / \mathrm{h}$. The three highways have homogenous physical conditions in terms of adjacent land use, shoulder type, and surface conditions. Lane and shoulder width is uniform and pavement surface is in fair condition without major distresses. The predominant traffic volumes on the three highways are low to moderate, according to the data of MTO. For example, the Annual Average Daily Traffic volume (AADT) on the experiment sections of Highway 7 was between 14,400 and 16,500 in 2004 (MTO, 2004).

The speed distribution for two-lane rural highways is presented in Table 5-2 and the corresponding results are also illustrated in Figure 5-3. Excluding the standstill time at 
intersections, the total travel time was 56,453 seconds spent by the 26 drivers on these roads. The maximum speed observed in the experiment reached $133 \mathrm{~km} / \mathrm{h}$, which considerably exceeded the speed limit of $80 \mathrm{~km} / \mathrm{h}$. Although the result of the one-sample t-test suggests that the mean speed of $80.98 \mathrm{~km} / \mathrm{h}$ for the mixed condition was significantly different from the speed limits of $80 \mathrm{~km} / \mathrm{h}$ at $5 \%$ level of significance, the difference was relatively small in magnitude. As on Highway 417, a large portion of the speeds selected by the drivers was greater than the posted speed. The pace speed on the two-lane rural highways was from 80 to $90 \mathrm{~km} / \mathrm{h}$.

According to Table 5-2, the test vehicle traveled under free-flow conditions for a majority of the time ( $78.5 \%$ of the time). The mean speeds for free-flow and non-freeflow conditions were close to each other, and both of them were close to the PSL. Nevertheless, the difference of two mean speeds was still proven to be statistically significant at $5 \%$ level of significance using the Independent-Samples t-test in SPSS. Additionally, the mean speed for free-flow conditions was checked to be significantly different from the posted speed of $80 \mathrm{~km} / \mathrm{h}$ at $5 \%$ level of significance, whereas the one for non-free-flow conditions was not statistically different from the PSL. As shown in Figure 5-3, both free-flow and non-free-flow speeds had a large portion in high speed ranges (i.e., greater than $70 \mathrm{~km} / \mathrm{h}$ ); it implies that the drivers were able to travel at high speeds in light traffic conditions even if the time headways were less than 5 seconds, as the leading vehicles were also adopting high speeds. This observation was supported by the video for the test runs. 


\begin{tabular}{|c|c|c|c|c|c|c|c|c|c|c|c|c|c|c|c|c|c|}
\hline \multirow{5}{*}{ 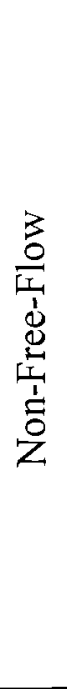 } & $a e^{2}$ & 웅 & 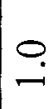 & $?$ & $\vec{F}$ & $\stackrel{c}{n}$ & $\stackrel{\nabla}{\circ}$ & $\dot{\nabla}$ & $\dot{\Xi}$ & $\mid \begin{array}{c}0 \\
m \\
n \\
n\end{array}$ & 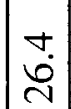 & $\left|\begin{array}{l}\infty \\
\dot{+}\end{array}\right|$ & 9 & $\tilde{0}$ & $\stackrel{0}{8}$ & \multirow{5}{*}{ 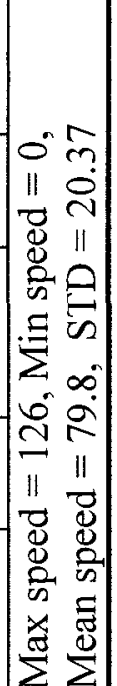 } & \\
\hline & $z e$ & 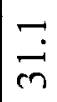 & $\frac{n}{2}$ & $\stackrel{\sim}{d}$ & 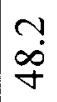 & $\ddot{m}$ & तi & $\begin{array}{c}0 \\
\infty \\
-\end{array}$ & $\begin{array}{l}n \\
n \\
n\end{array}$ & 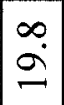 & $\begin{array}{l}0 \\
\text { กे }\end{array}$ & $\begin{array}{l}0 \\
\infty \\
\infty\end{array}$ & $\begin{array}{l}n \\
n \\
n\end{array}$ & $\stackrel{\forall}{ \pm}$ & $\frac{n}{n}$ & & \\
\hline & $z \stackrel{0}{0}$ & $\sqrt{2}$ & I & in & in & $\mid \begin{array}{l}\infty \\
\infty \\
m\end{array}$ & $\frac{\nabla}{8}$ & $\left|\begin{array}{l}\infty \\
0 \\
0 \\
n\end{array}\right|$ & $\stackrel{\infty}{\infty}$ & $\begin{array}{l}\bar{\sigma} \\
0 \\
\gamma^{\prime}\end{array}$ & 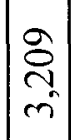 & $\begin{array}{l}0 \\
\infty \\
n \\
n\end{array}$ & त̂ & in & $\frac{g}{\mathfrak{g}}$ & & \\
\hline & en & 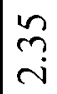 & $\hat{\infty}$ & $\tilde{\alpha}$ & $b_{j}$ & $\begin{array}{l}n \\
0 \\
n\end{array}$ & $\begin{array}{l}\infty \\
\infty \\
i\end{array}$ & $\tilde{a}$ & ri & $\begin{array}{l}\infty \\
i \\
i\end{array}$ & $\begin{array}{l}\varnothing \\
\infty \\
\text { i }\end{array}$ & $\begin{array}{c}\sigma \\
\sigma \\
i\end{array} \mid$ & $\left|\begin{array}{l}\multirow{b}{0}{} \\
\text { i }\end{array}\right|$ & g & & & \\
\hline & 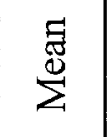 & $\vec{i}$ & $\begin{array}{l}3 \\
\infty \\
\dot{J}\end{array}$ & à & $\stackrel{N}{m}$ & $\stackrel{?}{\stackrel{P}{y}}$ & in & ๙2 & $\mid \begin{array}{c}\tilde{y} \\
\dot{\sigma} \\
r\end{array}$ & $\begin{array}{l}\tilde{a} \\
\dot{+} \\
\infty\end{array}$ & $\begin{array}{l}n \\
n \\
2 \\
2\end{array}$ & $\begin{array}{l}0 \\
\dot{0} \\
\dot{0}\end{array}$ & $\begin{array}{l}\sim \\
\Re \\
\sim \\
=\end{array}$ & $\frac{\text { I }}{\text { İ }}$ & & & \\
\hline \multirow{5}{*}{ 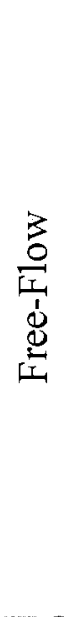 } & 0 尺 & $\ddot{\circ}$ & $\tilde{0}$ & 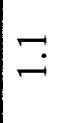 & 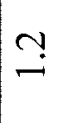 & 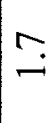 & $m$ & $\begin{array}{l}9 \\
\dot{n}\end{array}$ & $\begin{array}{c}0 \\
\text { Ni } \\
\text { in }\end{array}$ & $\ddot{\theta}$ & $\frac{1}{2}$ & $\begin{array}{l}0 \\
0\end{array}$ & $\begin{array}{l}\infty \\
\sim \\
\sim\end{array}$ & $\stackrel{\infty}{0}$ & $\ddot{8}$ & \multirow{5}{*}{ 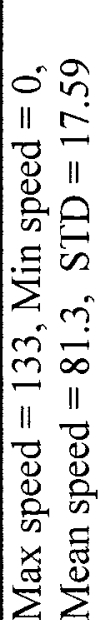 } & \\
\hline & $\frac{1}{a}$ e & $\hat{\infty}$ & $\stackrel{n}{n}$ & $\begin{array}{l}\infty \\
i \\
n\end{array}$ & $\frac{\infty}{i}$ & ? & $\frac{9}{2}$ & $\left|\begin{array}{c}0 \\
\text { j. } \\
\infty\end{array}\right|$ & $\begin{array}{l}n \\
\dot{+} \\
\infty\end{array}$ & $\mid \begin{array}{l}n \\
0 \\
\infty\end{array}$ & $\stackrel{+}{\dot{0}}$ & $\infty$ & $\mid \begin{array}{l}\infty \\
\dot{+} \\
\infty\end{array}$ & $\begin{array}{l}0 \\
\infty \\
\infty\end{array}$ & $\infty$ & & 咅 \\
\hline & Z & 悉 & $\begin{array}{c}0 \\
m \\
m\end{array}$ & $\stackrel{n}{q}$ & 交 & $\hat{6}$ & $i_{0}^{n}$ & $\mid$ & $\left|\begin{array}{l}m \\
2 \\
a\end{array}\right|$ & $\mid$\begin{tabular}{l}
$n$ \\
\multirow{2}{*}{} \\
$\sigma^{\prime}$
\end{tabular} & $\mid \begin{array}{l}\infty \\
\text { ஸ్ } \\
\sim \\
\sim\end{array}$ & $\mid$ & 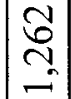 & $\bar{m}$ & $\begin{array}{l}0 \\
0 \\
0 \\
j \\
j\end{array}$ & & 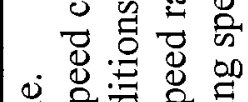 \\
\hline & $\hat{\theta}_{\infty}$ & $\begin{array}{l}n \\
\\
\end{array}$ & $\bar{a}$ & $\hat{\tilde{N}}$ & $\begin{array}{l}\infty \\
\infty \\
j\end{array}$ & \begin{tabular}{|l|}
$n$ \\
$\infty$ \\
$i$
\end{tabular} & $\begin{array}{l}\mathfrak{N} \\
\infty \\
\sim\end{array}$ & \begin{tabular}{|l|}
0 \\
$\infty$ \\
ij \\
\end{tabular} & $\frac{i}{i}$ & $\frac{i}{i}$ & $\frac{n}{r}$ & $\underset{|c|}{i}$ & 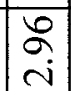 & $\stackrel{\infty}{\stackrel{\infty}{m}}$ & & & 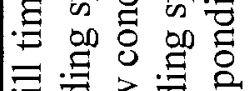 \\
\hline & 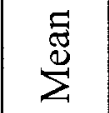 & in & $\begin{array}{l}\infty \\
0 \\
\dot{J}\end{array}$ & $\frac{\tilde{d}}{\dot{d}}$ & $\bar{\sigma}$ & $\begin{array}{l}\infty \\
\infty \\
\dot{j}\end{array}$ & 8 & 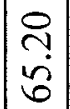 & $\begin{array}{l}\vec{f} \\
\dot{r} \\
\end{array}$ & $\begin{array}{l}8 \\
8 \\
\dot{1} \\
\infty\end{array}$ & $\frac{5}{2}$ & $\begin{array}{l}\infty \\
\infty \\
\infty \\
0 \\
0\end{array}$ & $\begin{array}{l}\overrightarrow{0} \\
\dot{\Xi} \\
\end{array}$ & $\begin{array}{l}\infty \\
\infty \\
\sim \\
\end{array}$ & & & 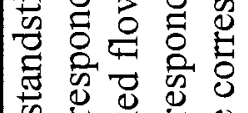 \\
\hline \multirow{5}{*}{ 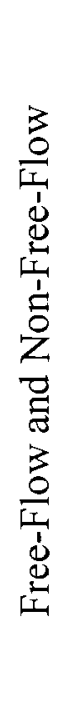 } & 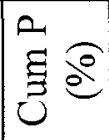 & $\tilde{0}$ & $\because$ & $\bar{i}$ & $\because$ & n & 19 & $\stackrel{\nabla}{\sim}$ & $\begin{array}{l}\infty \\
\sim \\
n\end{array}$ & i & $\stackrel{\dot{2}}{\Omega}$ & $\begin{array}{l}0 \\
\dot{2}\end{array}$ & $\tilde{a}$ & $\stackrel{0}{8}$ & & \multirow{6}{*}{\multicolumn{2}{|c|}{ 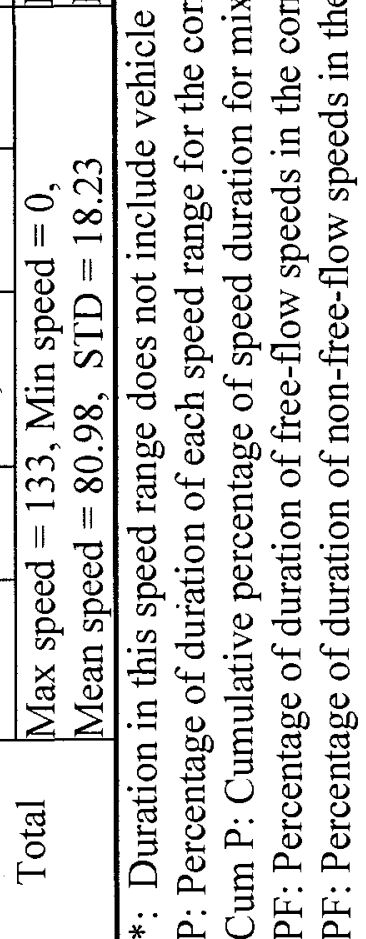 }} \\
\hline & -2 & $\tilde{0}$ & $\stackrel{\infty}{0}$ & $\stackrel{1}{-}$ & $\stackrel{\infty}{-}$ & i & $\stackrel{?}{m}$ & $\begin{array}{l}0 \\
\dot{v}\end{array}$ & $\begin{array}{l}\dot{\sigma} \\
\dot{0}\end{array}$ & $\ddot{m}$ & ֻே & $\vec{v}$ & $\begin{array}{l}0 \\
i\end{array}$ & $\dot{0}$ & $\stackrel{0}{8}$ & & \\
\hline & Z た & $\stackrel{\infty}{\infty}$ & in & in & î & $\stackrel{n}{=}$ & $\overbrace{\infty}^{\infty}$ & $\underset{\sigma}{\sigma}$ & $\underline{n}$ & 帒 & 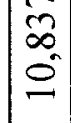 & 占 & 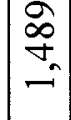 & $\stackrel{0}{\gamma}$ & $\begin{array}{l}3 \\
\text { fo } \\
0 \\
0\end{array}$ & & \\
\hline & $\theta_{n}$ & î & व & $\tilde{a}$ & $\infty$ & $\hat{a}$ & $\mid \begin{array}{l}\infty \\
\infty \\
i\end{array}$ & $\mid \begin{array}{l}\hat{\infty} \\
\tilde{\infty}\end{array}$ & $\frac{m}{r}$ & $\frac{m}{i}$ & $\stackrel{\hat{n}}{\tilde{N}}$ & $\stackrel{ }{i}$ & $\tilde{\sigma}$ & $\underset{\sigma}{\sigma}$ & & & \\
\hline & 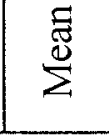 & $\begin{array}{l}\hat{6} \\
\dot{6}\end{array}$ & $\stackrel{N}{ \pm}$ & $\vec{a}$ & in & 汻 & $\frac{\sigma}{\dot{\sigma}}$ & $\begin{array}{l}\text { กิ } \\
\cdots \\
0\end{array}$ & $\begin{array}{l}n \\
f \\
r \\
r\end{array}$ & $\begin{array}{l}\widehat{\sigma} \\
\dot{\infty}\end{array}$ & $\stackrel{\infty}{\stackrel{\infty}{\sigma}}$ & 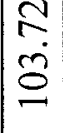 & $\begin{array}{l}\Omega \\
\tilde{2} \\
=\end{array}$ & 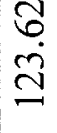 & & & \\
\hline $\begin{array}{l}\text { ठ্. } \\
\Phi \\
\text { क }\end{array}$ & 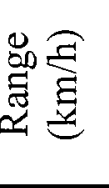 & $\begin{array}{l}0 \\
0 \\
v \\
v \\
0\end{array}$ & $\begin{array}{l}0 \\
\text { v } \\
3 \\
\text { vi } \\
0\end{array}$ & $\begin{array}{l}0 \\
\tilde{v} \\
\text { v } \\
\text { vi } \\
\text { v }\end{array}$ & P & 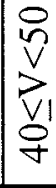 & 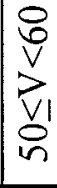 & $\begin{array}{l}8 \\
\text { v } \\
\vec{v} \\
8 \\
0\end{array}$ & $\begin{array}{c}0 \\
\infty \\
v \\
\vec{v} \\
0 \\
0\end{array}$ & $\begin{array}{l}0 \\
\hat{v} \\
\vec{v} \\
\text { vi } \\
\infty\end{array}$ & 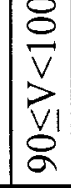 & 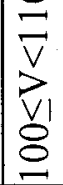 & $\begin{array}{l}\text { v } \\
\bar{v} \\
\vec{v} \\
0 \\
0 \\
=\end{array}$ & 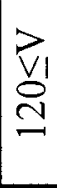 & & & \\
\hline
\end{tabular}




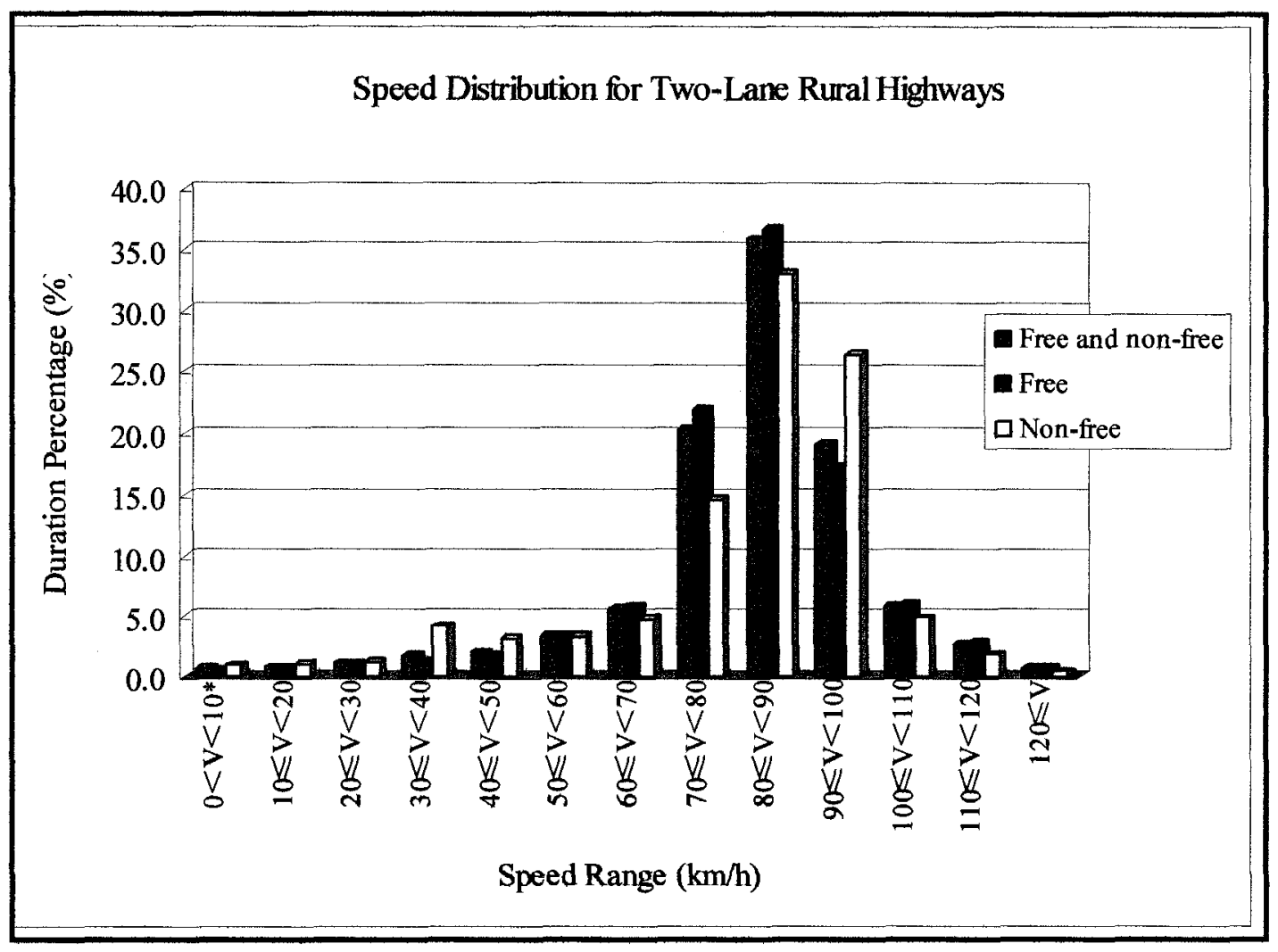

Figure 5-3: Speed Distribution for Two-Lane Rural Highways.

\subsubsection{Rural Freeway (Highway 416)}

Highway 416 is a typical rural freeway on which low to moderate traffic volumes predominate. For instance, the AADT on the experiment sections of this freeway was between 18,100 and 26,500 in 2004 (MTO, 2004). Similar to Highway 417, Highway 416 has a constant PSL of $100 \mathrm{~km} / \mathrm{h}$.

The speed distribution for Highway 416 is presented in Table 5-3 and the corresponding results are illustrated in Figure 5-4. A total of 13,797 seconds was elapsed on Highway 416 for the driver sample. The observed speeds ranged from 58 to $146 \mathrm{~km} / \mathrm{h}$, resulting in a mean speed of $107.18 \mathrm{~km} / \mathrm{h}$ for the mixed flow conditions. Unlike Highway 417 , this mean speed was substantially greater than the speed limit of $100 \mathrm{~km} / \mathrm{h}$. The difference between this mean speed and the speed limit was statistically significant at $5 \%$ 
level of significance, according to the result of one-sample t-test. The speed distribution for Highway 416 presented distinct characteristics from that for Highway 417. Most speeds chosen by the driver sample were higher than $90 \mathrm{~km} / \mathrm{h}$, which account for around 95 percent of the time on this freeway. A majority of the speeds were above the speed limit, and the pace speed was in the range between 100 and $110 \mathrm{~km} / \mathrm{h}$, which was same as that for Highway 417.

According to Table 5-3, speeds of free-flow conditions almost had the same duration as that of non-free-flow conditions. Interestingly, the mean speed for free-flow conditions was very close to, but slightly lower than, that for non-free-flow conditions. Although these two mean speeds were statistically different at $5 \%$ level of significance, the difference of the two mean speeds was relatively small (less than $0.5 \%$ ). For this case, the explanation is that driver speed choice under non-free-flow conditions might not be distinct from free-flow conditions when the traffic volumes were relatively low and drivers had chances to make a lane change to pass slow-moving vehicles in front. Therefore, the leading vehicles in light traffic conditions did not necessarily impede the trailing vehicle; rather the trailing vehicles with less than 5-second time headway were still traveling at speeds of drivers' choice. This explanation was supported by the video recorded in the test runs. 


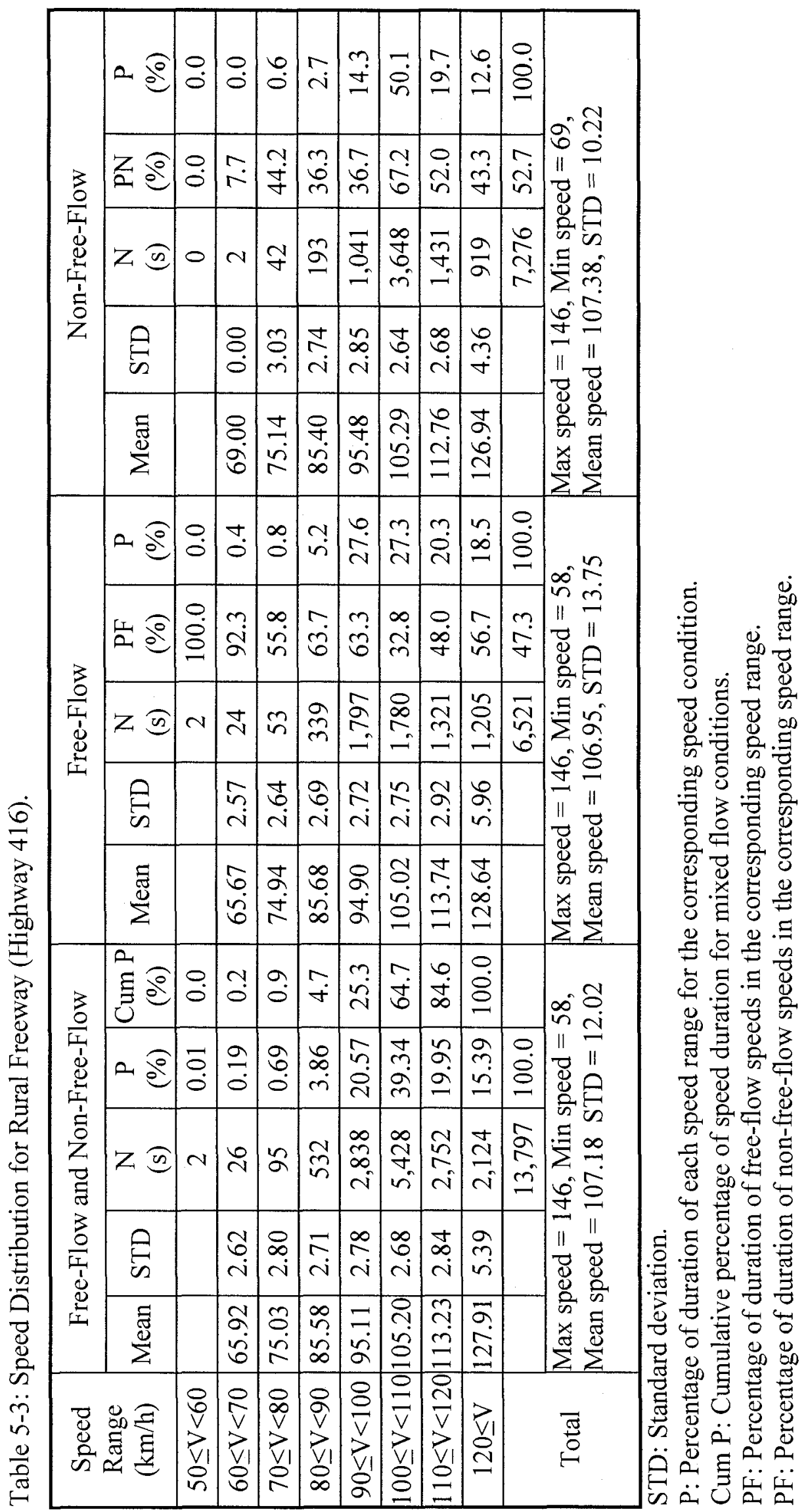




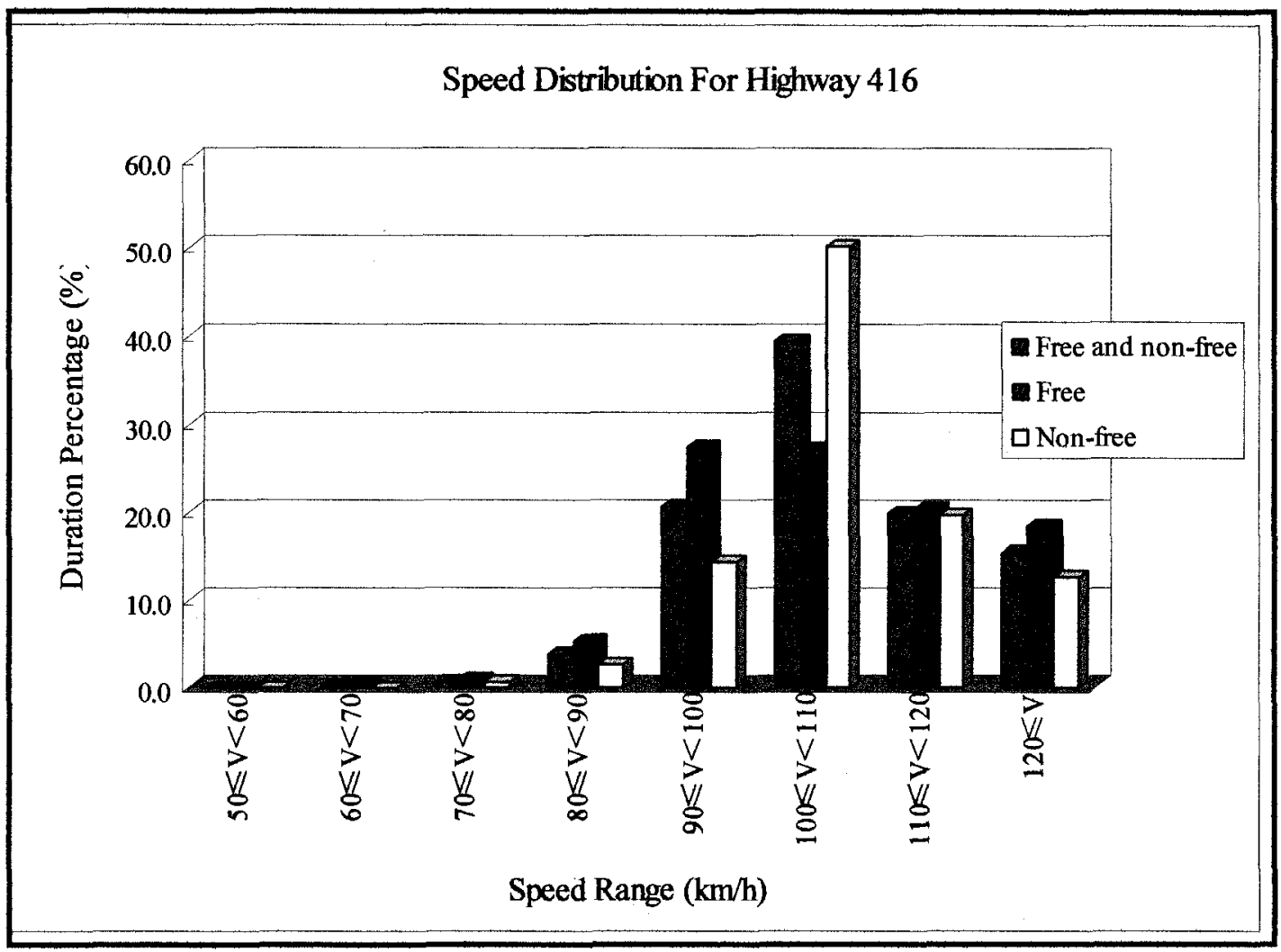

Figure 5-4: Speed Distribution for Rural Freeway (Highway 416).

\subsubsection{Urban/Suburban Roads}

Regional Routes 12 and 73 are typical undivided two-lane roads located near densely populated areas. Intersections appear more frequently than on the roads in rural areas. Various speed limits are posted on these two roads, including 50,60 and $80 \mathrm{~km} / \mathrm{h}$. The lane width is uniform and the pavement condition is fair on both roads.

The speed distribution for urban/suburban roads is presented in Table 5-4 and Figure 5-5. Similar to the analysis for the two-lane rural highways, the vehicle's standstill time was excluded from the trip duration, resulting in a total travel time of 24,279 seconds on these two roads. Because high density of intersections and high traffic volumes are expected on these urban/suburban roads, driving speeds were more fluctuant, as illustrated in Figure 5-5. The prevailing speed choice in the driver sample covered four 
speed ranges from 40 to $80 \mathrm{~km} / \mathrm{h}$. In addition, the drivers had little chance to travel at speeds higher than the upper speed limit of $80 \mathrm{~km} / \mathrm{h}$.

Based on the threshold of 3-second headway in distinguishing between free-flow and non-free-flow conditions, the free-flow speeds accounted for 39 percent of the travel time elapsed on the urban/suburban roads and non-free-flow speeds accounted for 61 percent. The mean speed for free-flow conditions was substantially higher than that for non-freeflow conditions. Furthermore, the difference between the two mean speeds was examined to be statistically different at $5 \%$ level of significance. As expected, free-flow speeds appeared more often in the high-speed ranges, whereas non-free-flow speeds occurred more frequently at low speeds, according to Figure 5-5. 


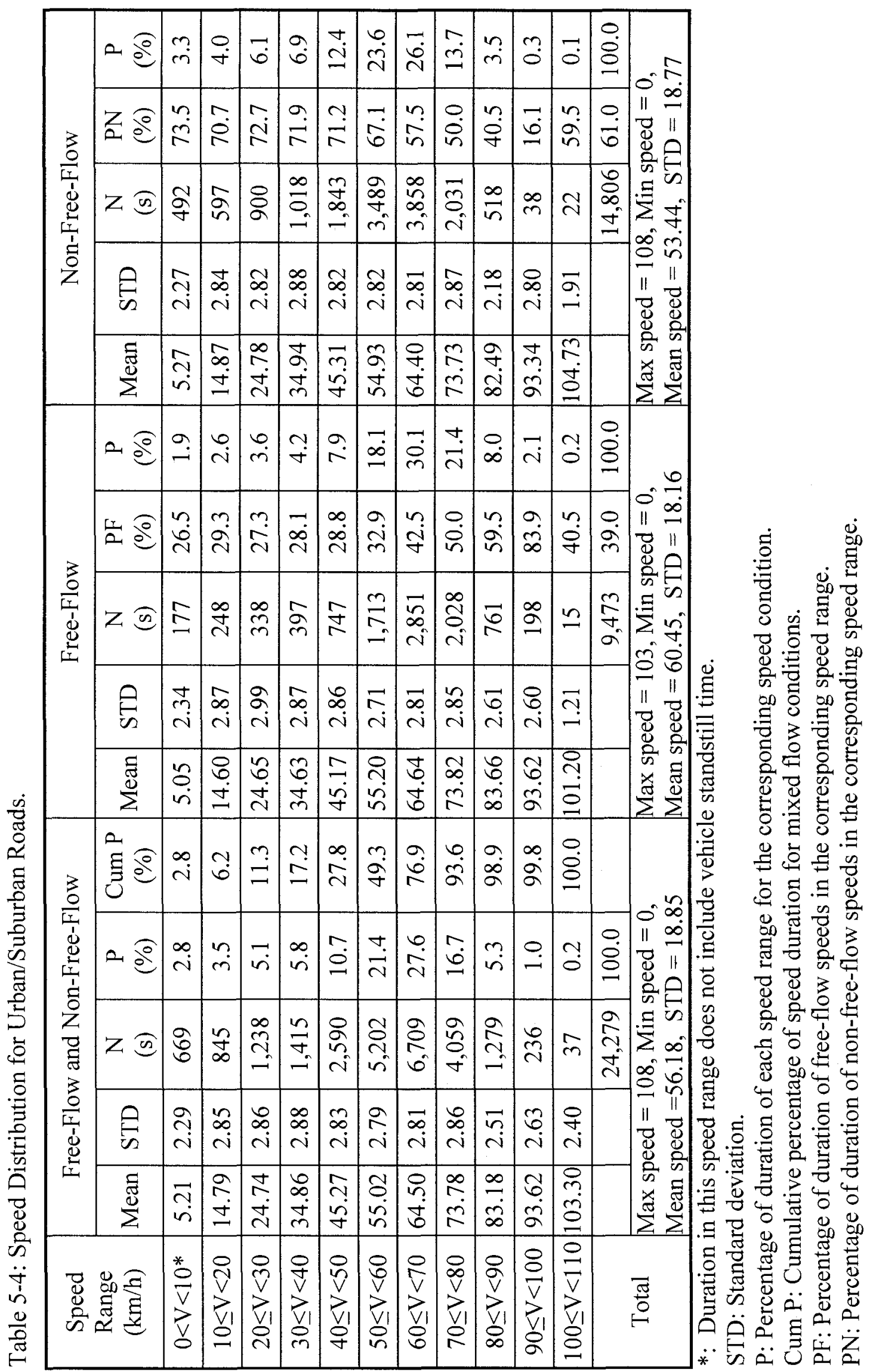




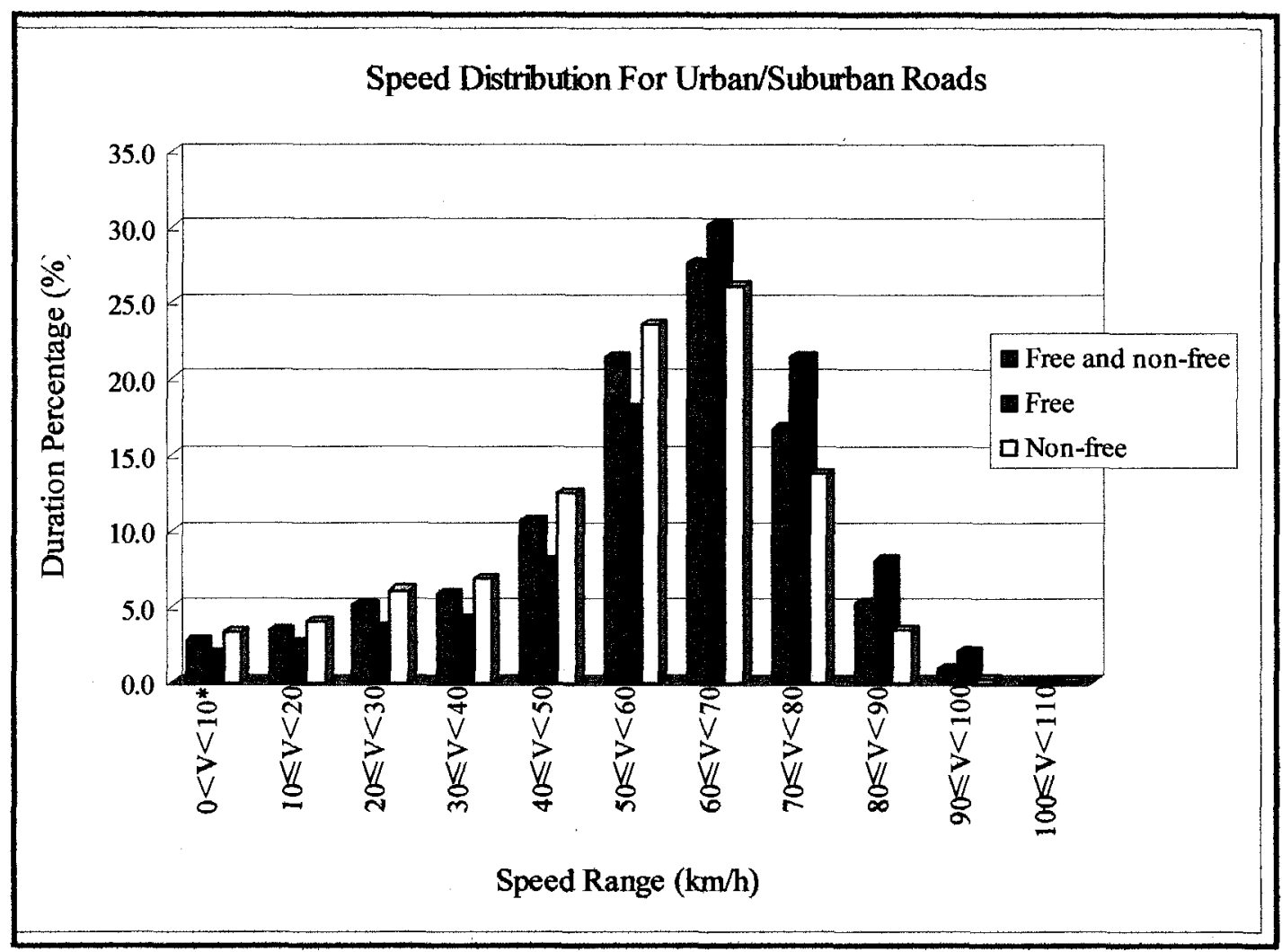

Figure 5-5: Speed Distribution for Urban/Suburban Roads.

\subsubsection{Summary of Driver Speed Behaviour on Different Road Classes}

The above analysis of speeds on individual road classes presents an essential understanding of driver speed behaviour under different driving environments. In order to provide a comprehensive recognition of driver speed behaviour across the diverse road classes, the statistics of the speed distributions on the four road classes as well as the posted speed limits on these roads are summarized in Table 5-5. Accordingly, the key observations are spelled out as follows: 


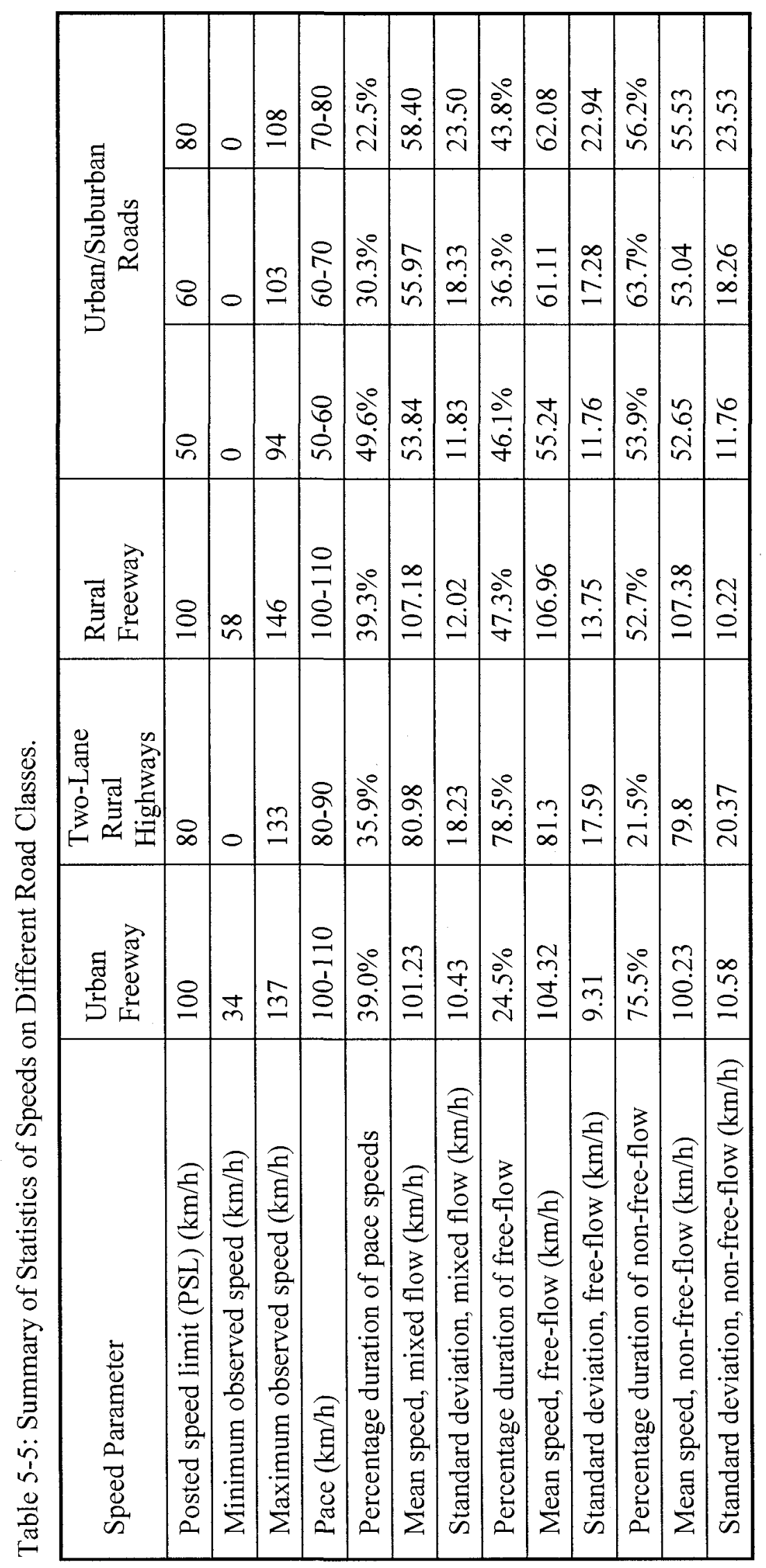


- The percentage of free-flow speeds on the different road types varied, which implies that traffic conditions and geometric characteristics on these roads were substantially different. For example, the portion of free-flow speeds on Highway 417 was less than 25 percent, whereas free-flow speeds were most common on two-lane rural highways. Generally, based on the proportions of free-flow duration, heavy traffic volumes were prevalent on the urban freeway and light traffic volumes were predominant on two-lane rural highways. One needs to be cautious, however, in comparing the percentage of time under free-flow conditions for urban/suburban roads to the other road classes due to the different criterion in distinguishing free-flow speed, as mentioned earlier.

- For the freeways and two-lane rural highways, the average speeds for both the mixed-flow conditions and free-flow conditions were all greater than the corresponding posted speed limits. The differences between these average speeds and their speed limits were tested to be statistically significant at $5 \%$ level of significance. However, this relationship between average speed and corresponding PSL varied on urban/suburban roads. As shown in Table 5-5, the average speeds under free-flow and mixed-flow conditions for PSL of $50 \mathrm{~km} / \mathrm{h}$ were greater than this PSL. However, those average speeds for PSL of $80 \mathrm{~km} / \mathrm{h}$ were much lower than $80 \mathrm{~km} / \mathrm{h}$.

- The average free-flow speeds were generally greater than the corresponding values for non-free-flow conditions, with the exception of rural freeway with the difference in that case being relatively small (less than $0.5 \%$ ). Given the fact that the multi-lane freeway with relatively low traffic volume allows for a lane 
change to avoid impediment by slow-moving front vehicles, it is more likely that drivers who traveled with less than 5-second time headway were still driving at the speed of their choice and were trailing the front vehicle by their choice. This explanation was supported by the visual examination of the drivers' view of the road as recorded by the video camera during the test runs.

- The pace speed, which denotes a speed range with the largest speed frequency, fell in the next speed range above speed limits in most cases. It implies that the driver sample was more likely to keep their speeds over the speed limit to a reasonable extent.

- Figures 5-6 and 5-7 illustrate the portion of speeds in three speed bins under free-flow conditions for each road class: (1) below the posted speed limit (PSL), (2) within $10 \mathrm{~km} / \mathrm{h}$ over PSL, and (3) more than $10 \mathrm{~km} / \mathrm{h}$ over PSL. Because of the different posted speed limits on the urban/suburban roads, the speed choice of the driver sample was separately analyzed in relation to the corresponding PSL on each road segment. It should be noted that the $10 \mathrm{~km} / \mathrm{h}$ increment over the PSL was selected because of the general public's perception that they will not be ticketed for driving within $10 \mathrm{~km} / \mathrm{h}$ over the speed limit. The figures show that drivers would normally exceed the posted speed limit if the traffic condition would allow them to do so, but they try to keep the speed within 10 $\mathrm{km} / \mathrm{h}$ over the speed limit. However, for the case of urban/suburban roads with PSL of $80 \mathrm{~km} / \mathrm{h}$, the relatively closely-spaced controlled intersections on those road segments were a major cause affecting driver's preference for applying high speeds (i.e., speed higher than PSL). 


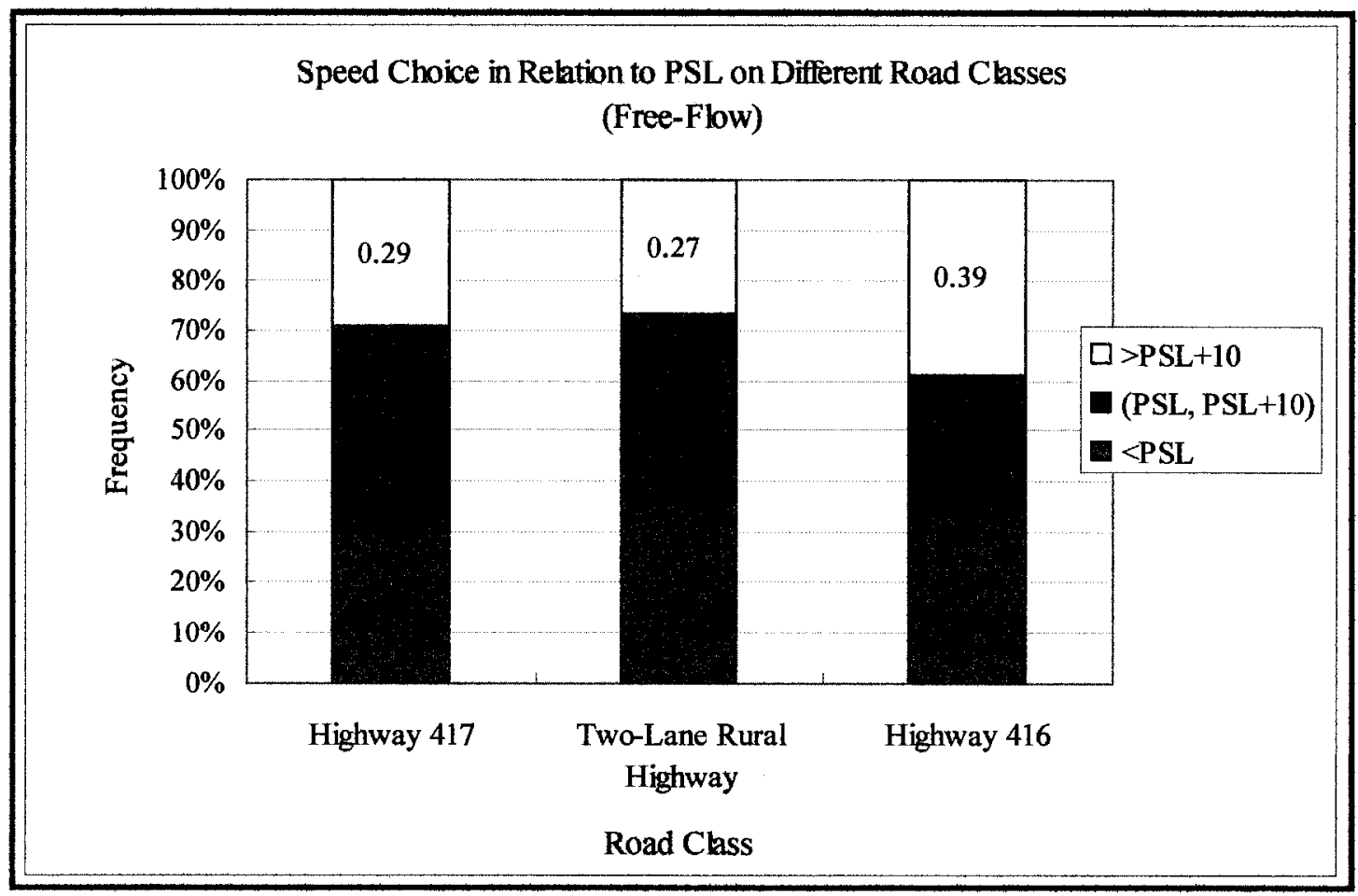

Figure 5-6: Speed Choice in Relation to PSL on Different Road Classes under Free-Flow Conditions.

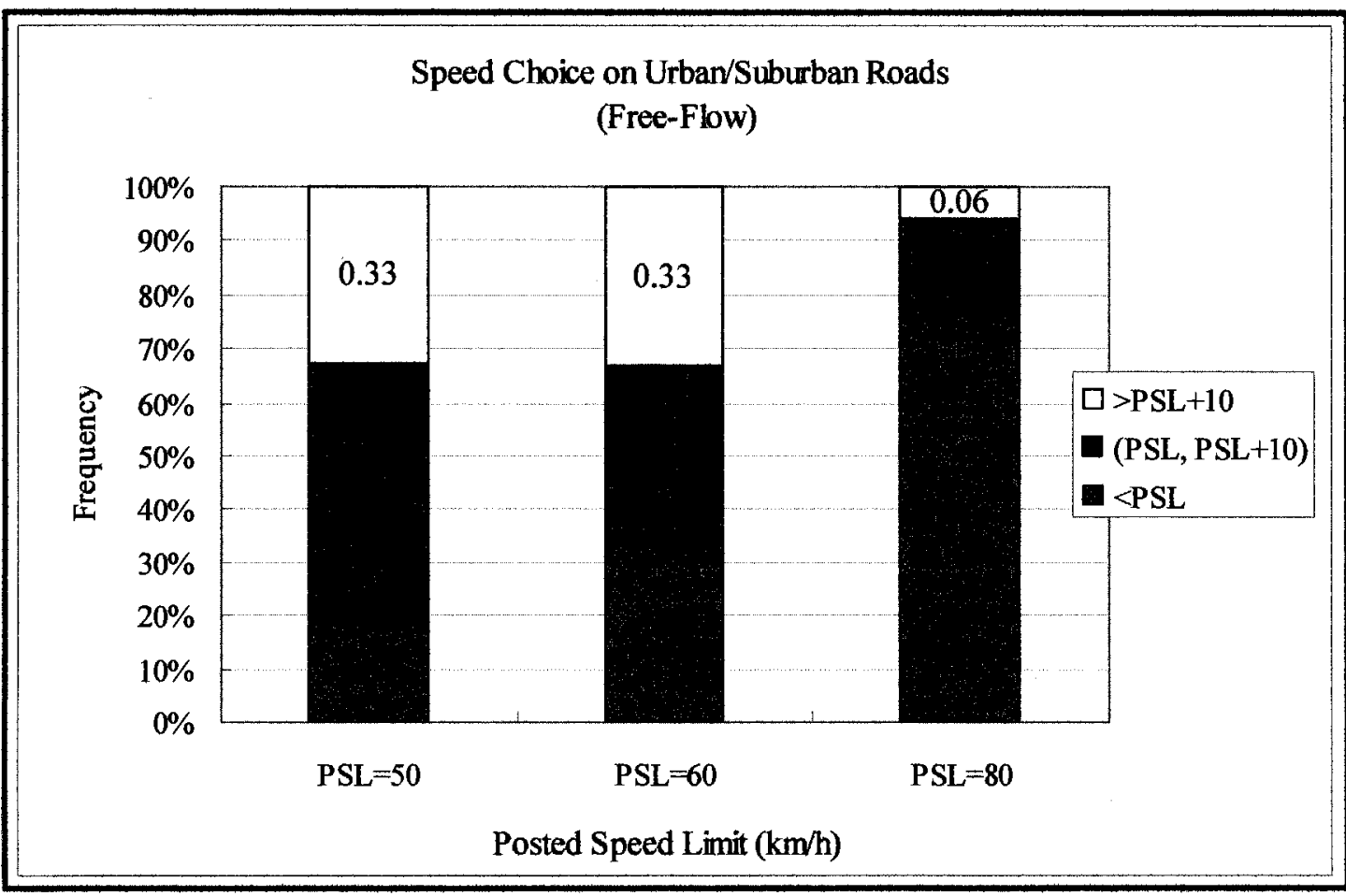

Figure 5-7: Speed Choice in Relation to PSL on Urban/Suburban Roads under Free-Flow Conditions. 


\subsection{Speed Behaviour of Different Driver Types}

In addition to the aggregate analysis of speed behaviour of the driver sample, another goal of this research was to attempt to categorize driver speed behaviour, in terms of aggressiveness, using the collected speed data. The speed choices of the drivers with various degree of aggressiveness were then analyzed in relation to speed limits on these four road classes. In this section, the procedure for categorizing driver speed behaviour is explained first and then the difference of speed choice among these driver types is discussed.

\subsubsection{Categorization of Driver Speed Behaviour}

Drivers' aggressive behaviour is usually related to a diversity of activities such as weaving in and out of traffic to go ahead, running red lights, showing discourteous gestures to others and so on (Shinar, 1998; Raub et al., 2002). However, in this research, drivers' aggressive behaviour is only defined for the cases when the drivers show an aggressive manner in speed choice according to their driving performance in the test runs. It is assumed that the driver sample in the experiment contained drivers of various degrees of aggressiveness; the drivers were classified into three driver types: aggressive, common, and defensive. In other words, if a driver often drove faster than other drivers in the sample, the driver was categorized as an aggressive driver. Accordingly, defensive drivers denote those who employed low speeds more often and common drivers represent the rest of drivers in the sample.

In distinguishing the three types of drivers, a statistical analysis of speed data was performed for each of the 26 drivers on every road class, under three different conditions: 
free-flow, non-free-flow and mixed conditions, respectively. The results are summarized in Table A-1 to Table A-12 in Appendix A. Since the mixed condition was the combination of free-flow and non-free-flow, only the statistical results of the free-flow and non-free-flow conditions were involved in distinguishing the driver types. With a combination of two speed conditions and four road classes, a total of eight categories was produced for distinguishing driver types.

The categorization procedure included three steps, which are illustrated in Figure 5-8 and explained subsequently. An example is given in Table 5-6, which presents the result of distinguishing driving behaviour on Highway 416 under non-free-flow conditions. Some rows are omitted from the table for brevity.

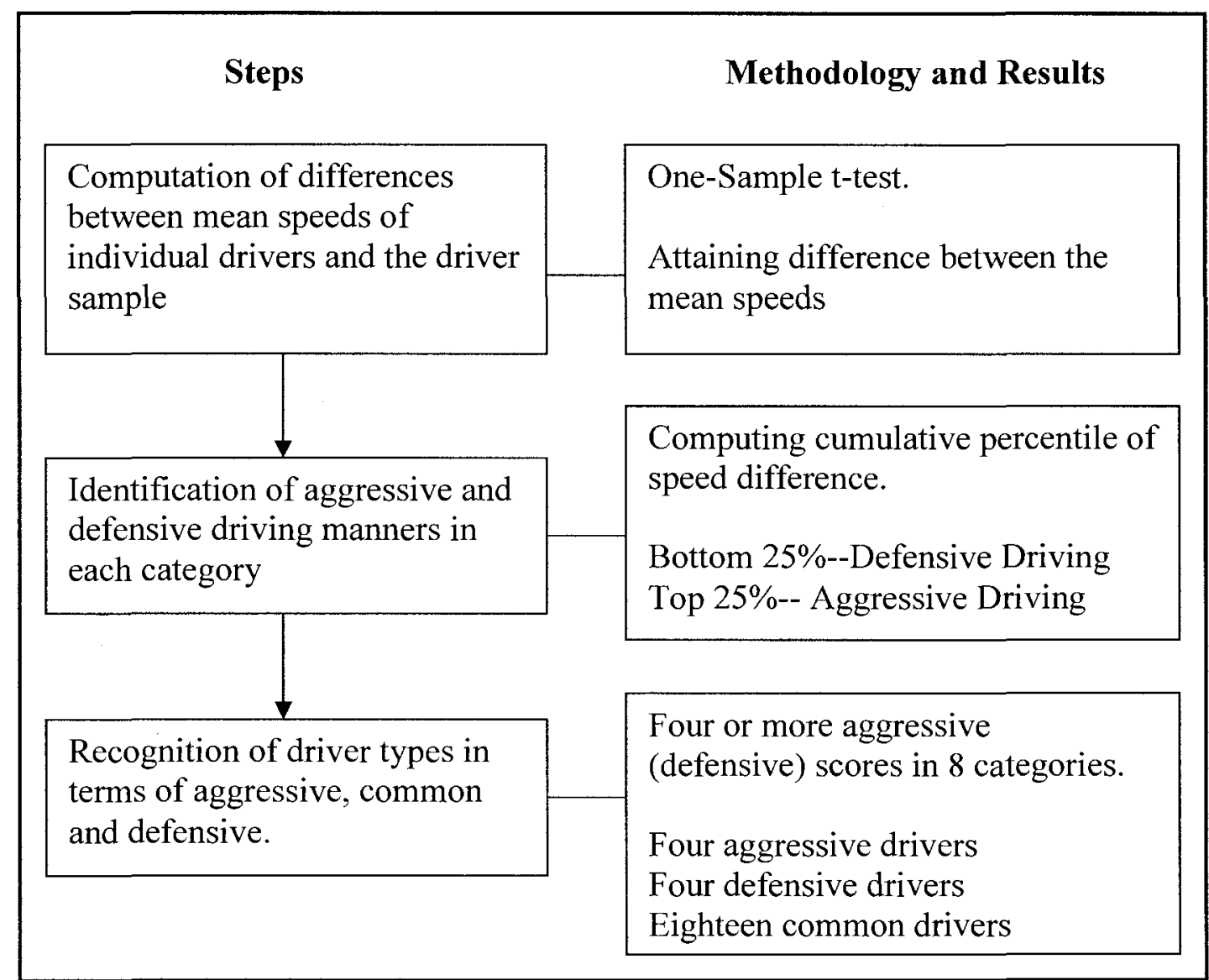

Figure 5-8: Procedure for Categorizing Driver Types. 


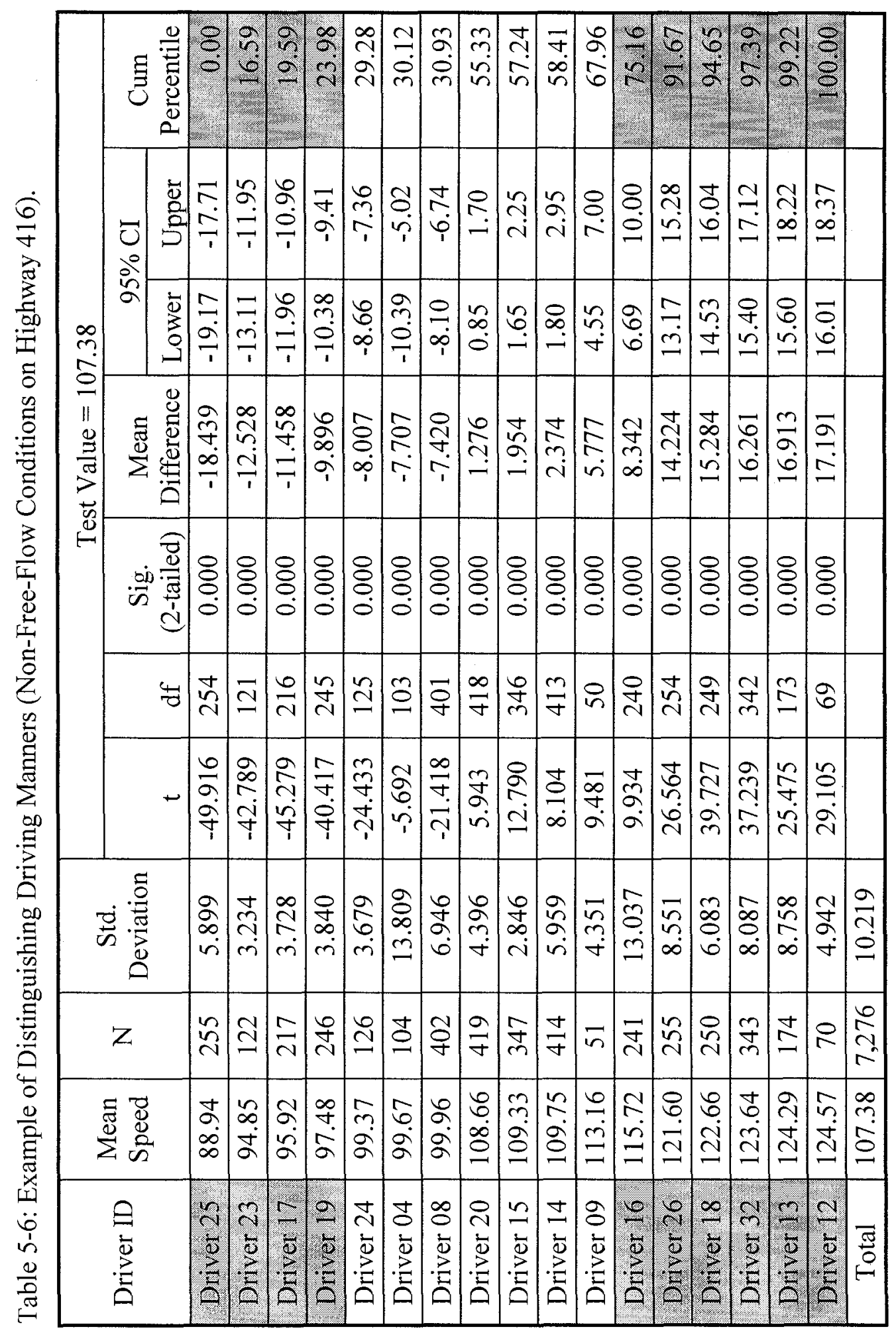


1. For each of the eight categories, the differences between the mean speeds of individual drivers and the driver sample were computed. The computation could be efficiently performed using the tool of one-sample t-test, producing an output which was readily available for further processing in the next step.

2. In each category, the calculated mean speed differences were ranked in an ascending order and their cumulative percentile was computed in the range of the speed difference. Accordingly, the driving manner of an individual driver was sorted as defensive if the corresponding difference was in the bottom 25 percentage of the sample's speed difference distribution; whereas the driving manner was considered aggressive if the difference was in the top 25 percentage. As shown in Table 5-6, four drivers, Drivers 25, 23, 17 and 19 in this example, showed relatively defensive driving manner on the rural freeway under non-freeflow conditions relative to the rest of the sample of drivers. On the other hand, six drivers in the top 25 percentage, Drivers $16,26,18,32,13$ and 12 , displayed relatively aggressive driving due to their highest average speeds.

3. After identification of the driving manner of the individual drivers for each category, the drivers who displayed four or more aggressive driving behaviours in the eight categories were recognized as aggressive drivers. Defensive drivers were also determined in a similar manner, and the remaining drivers were then sorted as common drivers. 
Table 5-7: Result of Judging Aggressive and Defensive Driving on the Four Road Classes.

\begin{tabular}{|c|c|c|c|c|c|c|c|c|}
\hline \multirow{2}{*}{ Driver ID } & \multicolumn{2}{|c|}{ Urban Freeway } & \multicolumn{2}{|c|}{$\begin{array}{c}\text { Two-lane } \\
\text { Rural Highways }\end{array}$} & \multicolumn{2}{|c|}{ Rural Freeway } & \multicolumn{2}{|c|}{$\begin{array}{l}\text { Urban/Suburban } \\
\text { Roads }\end{array}$} \\
\hline & Free & Non-Free & Free & Non-Free & Free & Non-Free & Free & Non-Free \\
\hline \multicolumn{9}{|l|}{ Driver 02} \\
\hline \multicolumn{9}{|l|}{ Driver 03} \\
\hline Driver 04 & & & & D & & & D & \\
\hline Driver 05 & & & & & & & $\mathrm{D}$ & \\
\hline Driver 06 & & & & $\bar{A}$ & & & & \\
\hline Driver 08 & & & & & & & $\mathrm{D}$ & $\bar{D}$ \\
\hline Driver 09 & $\mathrm{~A}$ & $\mathrm{~A}$ & & $\mathrm{~A}$ & & & & \\
\hline Driver 10 & & & & A & & & $\mathrm{D}$ & \\
\hline Driver 11 & & & & & & & & $\mathrm{D}$ \\
\hline Driver 12 & $\mathrm{~A}$ & $\mathrm{~A}$ & & $\mathrm{~A}$ & $\mathrm{~A}$ & $\mathrm{~A}$ & & A \\
\hline Driver 13 & & & $\mathrm{~A}$ & $\mathrm{~A}$ & $\mathrm{~A}$ & $\mathrm{~A}$ & & \\
\hline Driver 14 & $\mathrm{~A}$ & & & & & & $\mathrm{D}$ & \\
\hline Driver 15 & & & & & & & $\bar{D}$ & A \\
\hline Driver 16 & $\mathrm{~A}$ & $\mathrm{~A}$ & & $\mathrm{~A}$ & & $\mathrm{~A}$ & & \\
\hline Driver 17 & & & $\mathrm{D}$ & $\mathrm{D}$ & $\mathrm{D}$ & $\mathrm{D}$ & $\mathrm{D}$ & \\
\hline Driver 18 & $\mathrm{~A}$ & & & & & $\mathrm{~A}$ & $\mathrm{~A}$ & \\
\hline Driver 19 & & $\mathrm{D}$ & $\mathrm{D}$ & & D & D & & \\
\hline Driver 20 & & & & & & & & A \\
\hline Driver 21 & & & & & & & $\mathrm{D}$ & \\
\hline Driver 22 & & $\mathrm{D}$ & & & & & & \\
\hline Driver 23 & $\mathrm{D}$ & $\mathrm{D}$ & $\mathrm{D}$ & & $\mathrm{D}$ & D & $\bar{D}$ & \\
\hline Driver 24 & & & $\mathrm{D}$ & & $\mathrm{D}$ & & & $\mathrm{D}$ \\
\hline Driver 25 & & & $\mathrm{D}$ & $\mathrm{D}$ & $\mathrm{D}$ & $\mathrm{D}$ & $\mathrm{D}$ & $\mathrm{D}$ \\
\hline Driver 26 & & & & $\mathrm{~A}$ & A & A & & \\
\hline Driver 31 & & & & & $\mathrm{D}$ & & & $\mathrm{A}$ \\
\hline Driver 32 & & & $\mathrm{~A}$ & & $\mathrm{~A}$ & $\mathrm{~A}$ & $\mathrm{~A}$ & $\mathrm{~A}$ \\
\hline
\end{tabular}
A: Aggressive driving
D: Defensive driving. 
Following this procedure, all drivers were successfully categorized based on their speed performance, as shown in Table 5-7. Four defensive drivers (Drivers 17, 19, 23 and 25 ) and four aggressive drivers (Drivers 12, 13, 16 and 32) were identified from the 26 drivers in the sample, and thus the remaining 18 drivers were considered as common ones.

\subsubsection{Actual Driving Patterns versus Self-Reported Driving Patterns}

As mentioned earlier, every recruited driver completed a survey in which they evaluated their own driving pattern according to five general categories: extremely cautious, moderately cautious, slightly cautious, slightly aggressive, or aggressive. On the basis of their actual driving performance in the experiment, the drivers were sorted into three types in terms of defensive, common, and aggressive. In order to check if drivers' actual driving patterns were in accordance with their reports, an effort is presented here to compare their actual performance with the reported pattern. The results are shown in Table 5-8 and Figure 5-9.

Table 5-8: Comparison of Self-Reported and Actual Driving Patterns.

\begin{tabular}{|l|c|c|c|c|}
\hline \multicolumn{2}{|c|}{ Self-Reported } & \multicolumn{3}{c|}{ Actual Driving Performance } \\
\hline Driving Pattern & Frequency & Defensive & Common & Aggressive \\
\hline Extremely Cautious & 3 & 1 & 2 & 0 \\
\hline Moderately Cautious & 16 & 1 & 11 & 4 \\
\hline Slightly Cautious & 3 & 0 & 3 & 0 \\
\hline Slightly Aggressive & 3 & 2 & 1 & 0 \\
\hline Aggressive & 1 & 0 & 1 & 0 \\
\hline Total & 26 & 4 & 18 & 4 \\
\hline
\end{tabular}




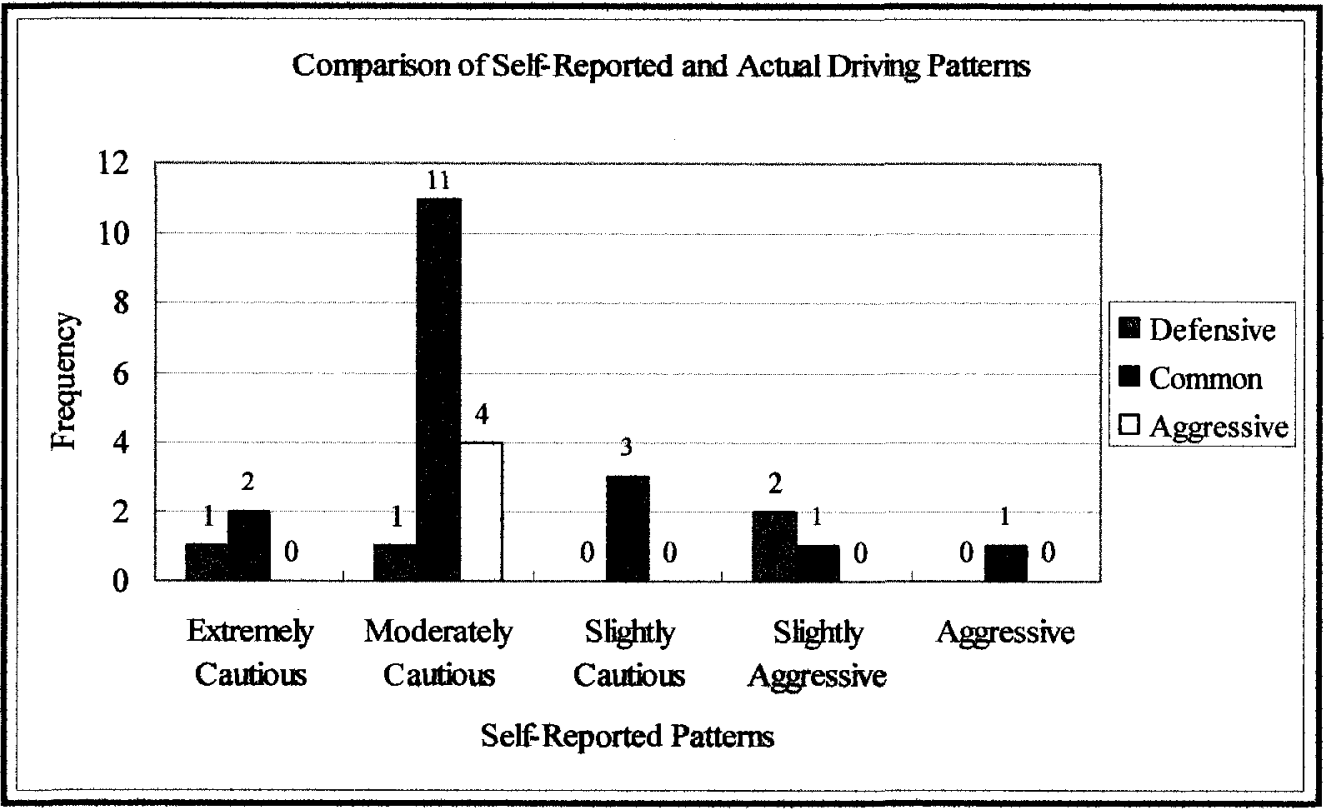

Figure 5-9: Comparison of Self-Reported and Actual Driving Patterns.

According to Table 5-8 and Figure 5-9, there was a considerable deviation of driver's actual driving performance from their self-reported driving pattern. All four identified aggressive drivers reported that they were moderately cautious in the survey, whereas two of the four defensive drivers evaluated themselves as slightly aggressive drivers. The common drivers accounted for the majority of the driver sample. Although the performance of certain common drivers was in accordance with their reported driving pattern, some of them still made a different self-reported evaluation. In summary, the drivers' self-reported evaluation does not necessarily demonstrate their actual driving pattern.

\subsubsection{Speed Choice of the Three Driver Types}

After individual drivers had been classified into three types based on their actual driving performance in the experiment, a further analysis was undertaken to investigate their speed choice in relation to the speed limits on different road classes. In order to 
eliminate the influence of traffic factors, the analysis is only performed under free-flow conditions.

For the purpose of this analysis, the speed distributions of every road class were produced for each driver type for the free-flow conditions. Based on the speed distributions, bar charts were plotted for these driver types to depict the relationship between the speed choices of the three driver types and the posted speed limits. The observed speeds were categorized into the same three speed bins discussed earlier: (1) less than PSL, (2) greater than PSL but less than PSL+10 km/h, and (3) greater than PSL $+10 \mathrm{~km} / \mathrm{h}$. The analysis results are presented for the four road classes individually.

\subsection{3.a Urban Freeway (Highway 417)}

Table 5-9 displays the speed distribution for the three driver types on Highway 417 under free-flow conditions, and Figure 5-10 illustrates the relationship of speed choice of these driver types with the PSL of $100 \mathrm{~km} / \mathrm{h}$. According to Table $5-9$ and Figure $5-10$, the aggressive drivers traveled above PSL for nearly 80 percent of the time, and almost half of their speeds exceeded PSL by more than $10 \mathrm{~km} / \mathrm{h}(49 \%$ of the time). On the other hand, the defensive drivers selected most of their speeds below the speed limit $(74 \%$ of the time), and they seldom drove at speeds exceeding speed limit by $10 \mathrm{~km} / \mathrm{h}$ or more $(1 \%$ of the time) even though they had chances to do so. In contrast, the common drivers also drove at speeds above PSL for a majority of the time (83\%). However, the portion of speeds exceeding PSL by $10 \mathrm{~km} / \mathrm{h}$ or more for the common drivers was substantially less than that for the aggressive drivers (14\% difference). 


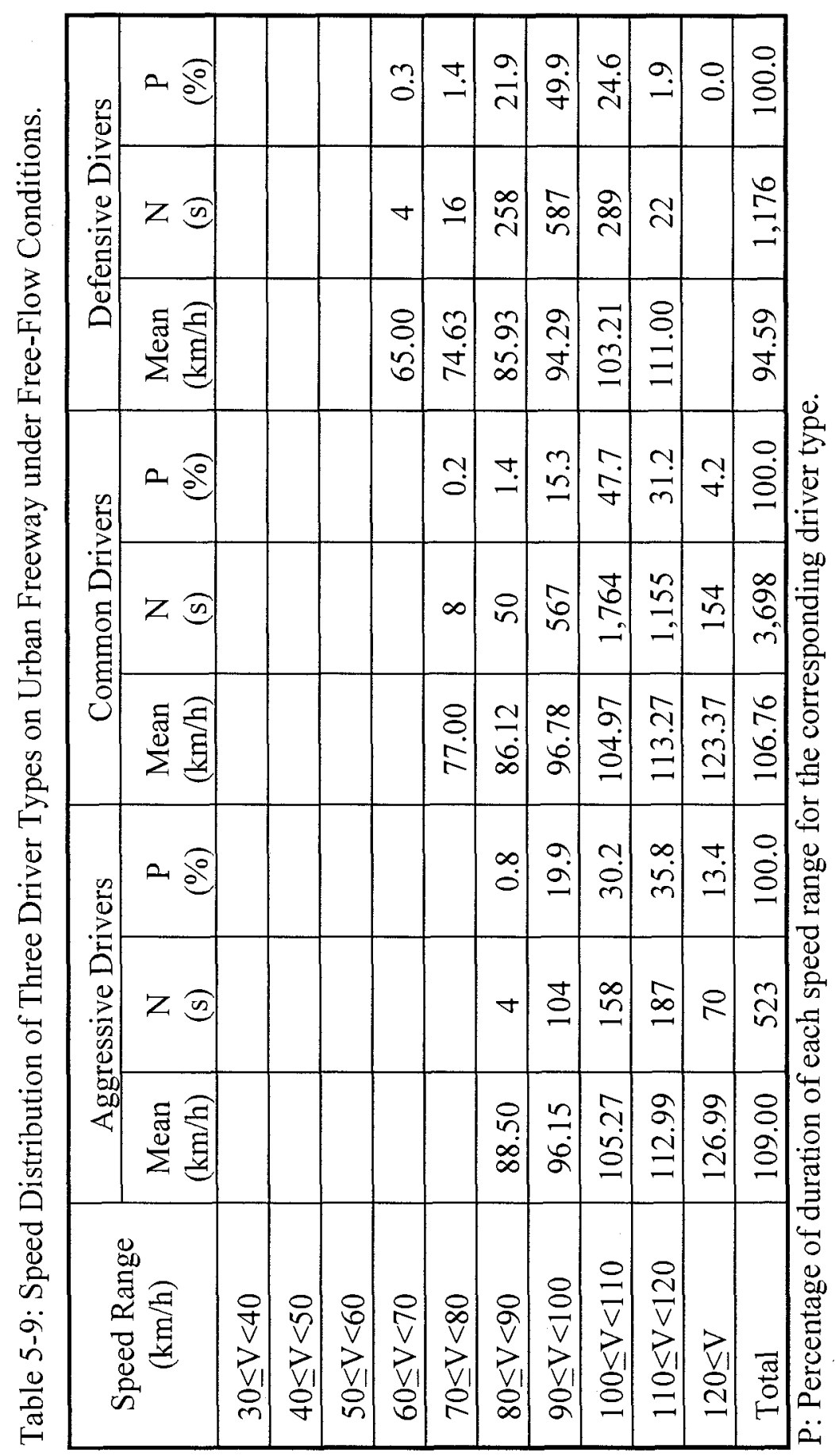




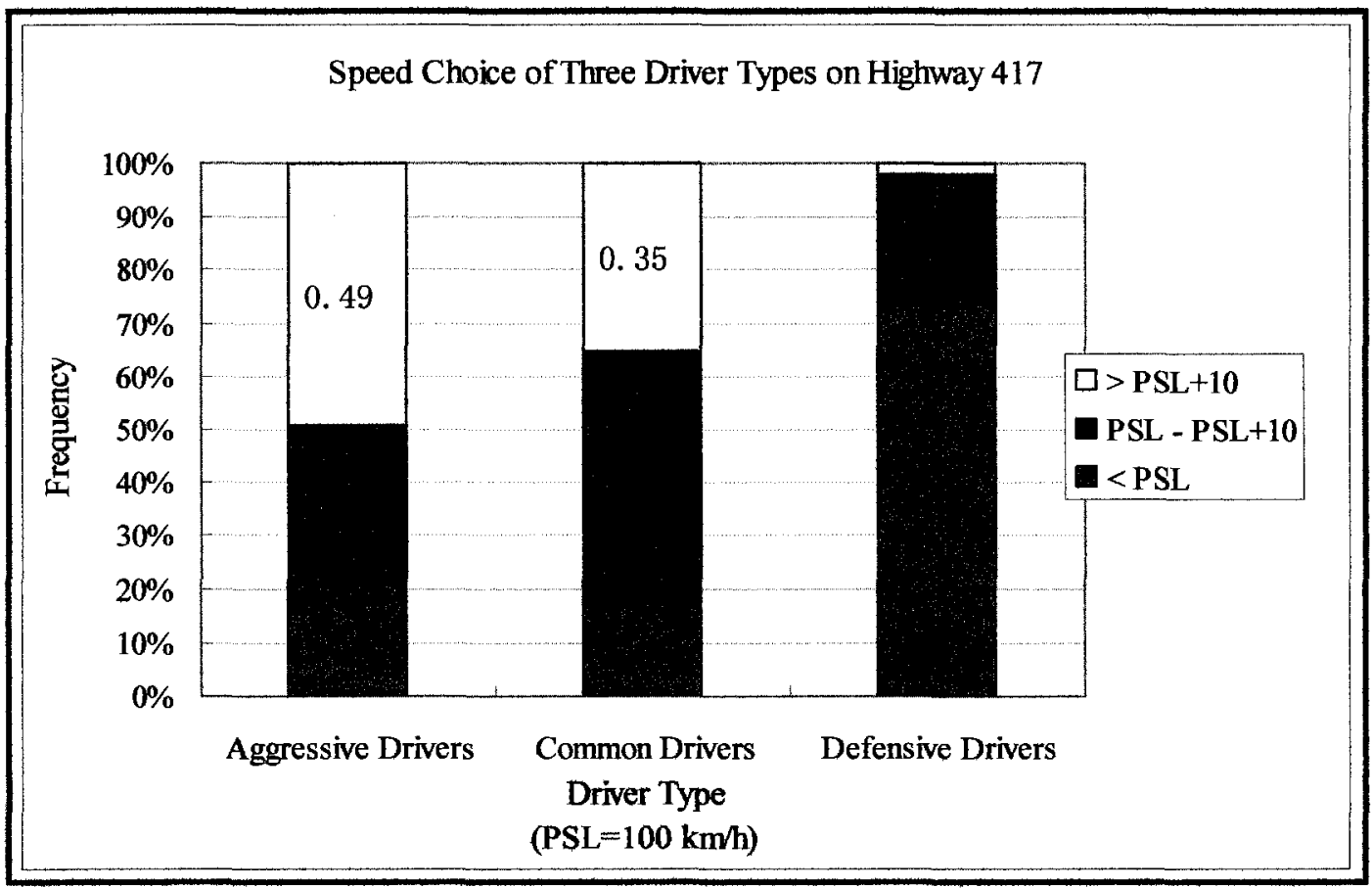

Figure 5-10: Speed Choice of Three Driver Types on Urban Freeway under Free-Flow Conditions.

\subsection{3.b Two-Lane Rural Highways}

Table 5-10 shows the distribution of free-flow speeds for the three types of drivers on two-lane rural highways, and Figure 5-11 presents the speed choice of these driver types in relation to the speed limit of $80 \mathrm{~km} / \mathrm{h}$ on the these roads. As shown in Figure $5-11$, the aggressive drivers exceeded the PSL by more than $10 \mathrm{~km} / \mathrm{h}$ for most of their trip duration under free-flow conditions ( $69 \%$ of the time). On the other hand, the defensive drivers traveled at the speeds below the PSL for most of their time under free-flow conditions ( $76 \%$ of the time), and they seldom drove at speeds exceeding speed limits by $10 \mathrm{~m} / \mathrm{h}$ or more ( $3 \%$ of the free-flow time). At the mean time, the common drivers had intermediate speed choices between the aggressive and defensive drivers. 


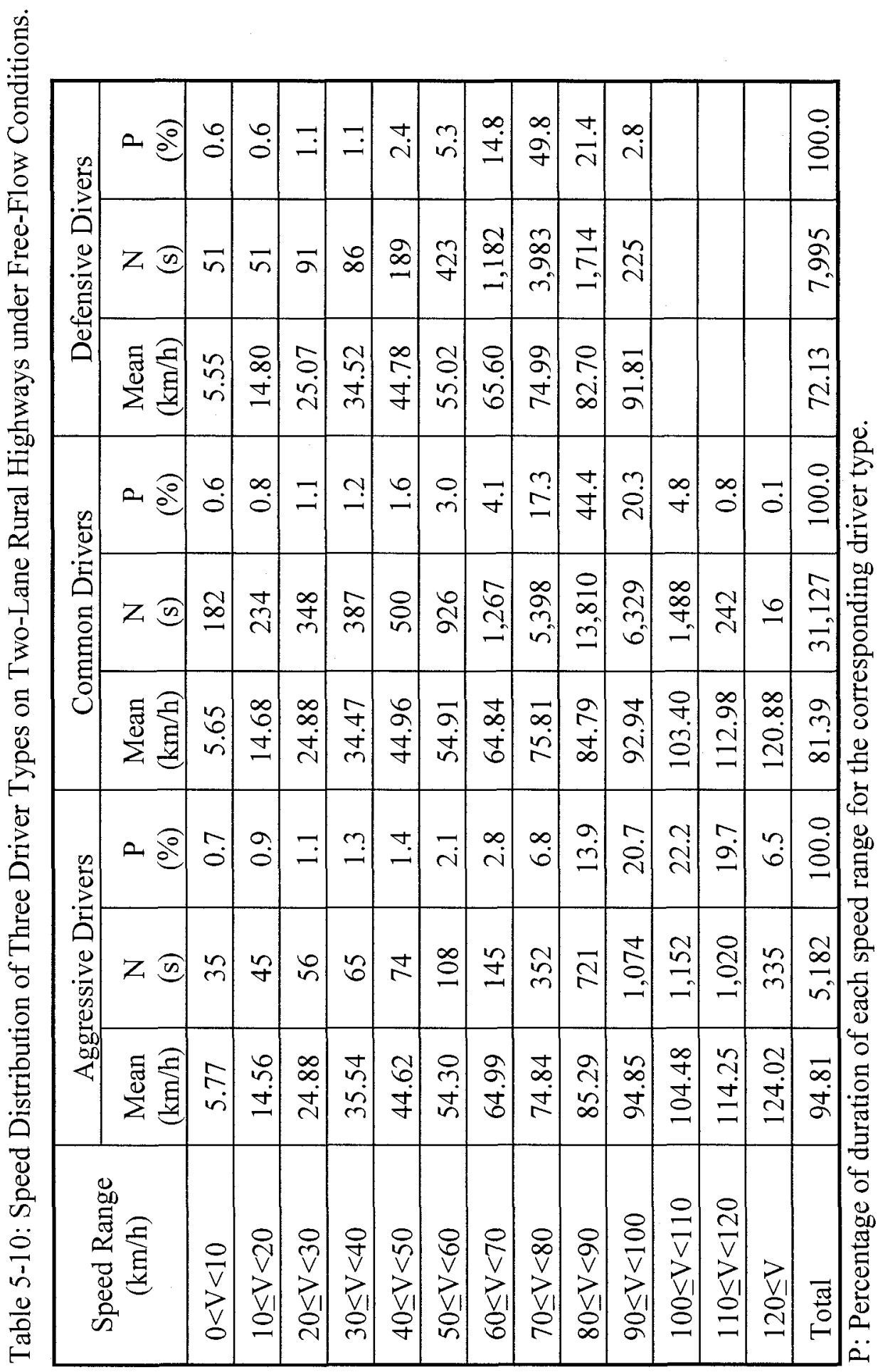




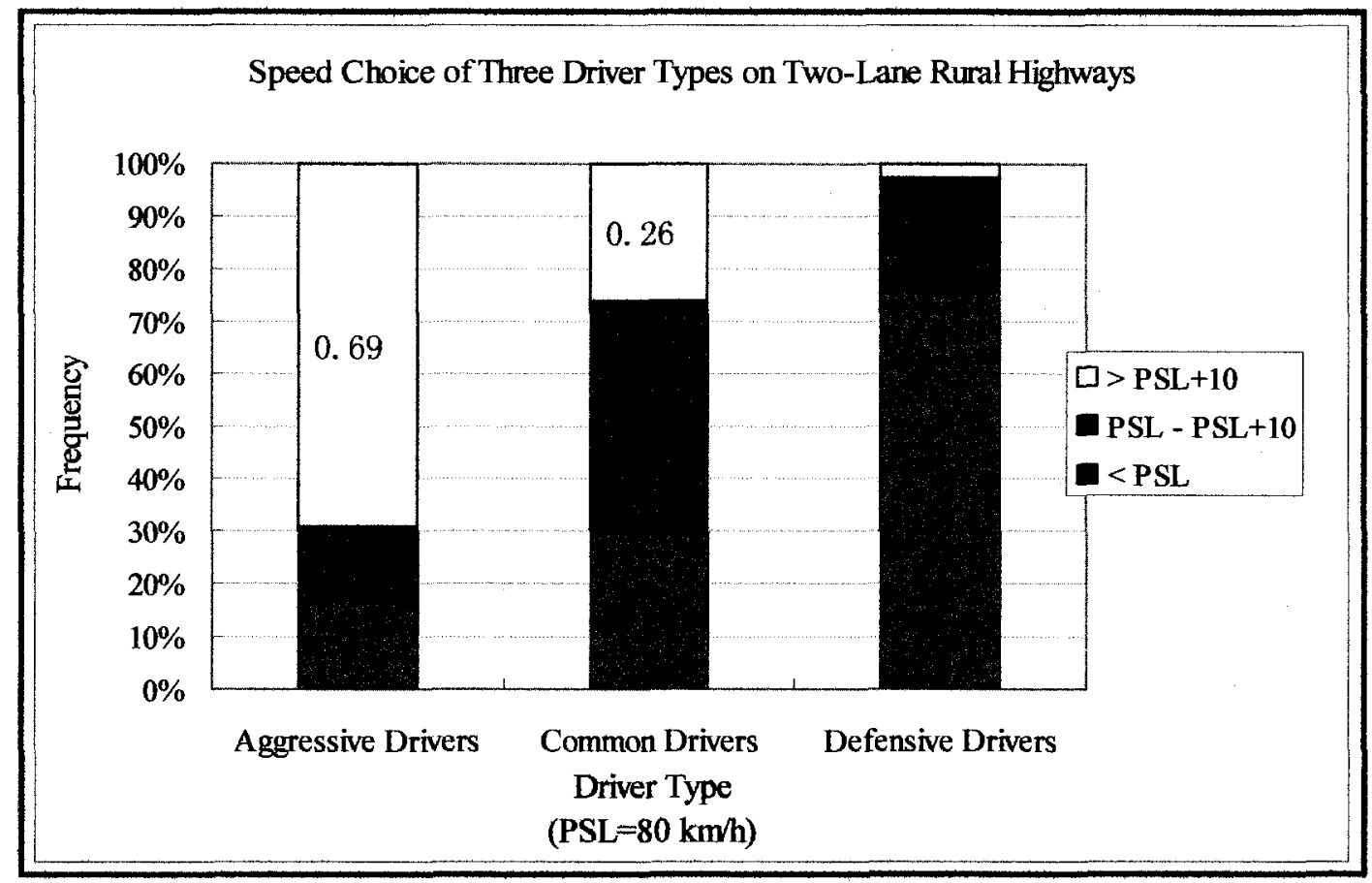

Figure 5-11: Speed Choice of Three Driver Types on Two-Lane Rural Highways under Free-Flow Conditions.

\subsection{3.c Rural Highway (Highway 416)}

Table 5-11 presents the distribution of free-flow speeds for the three driver types on Highway 416, and Figure 5-12 shows the relationship of speed choice of these driver types with the speed limit on Highway 416. As shown in Figure 5-12, the differences of speed choice between the three driver types became more evident. When the traffic condition was favourable, the aggressive drivers traveled at speeds above the PSL by 10 $\mathrm{km} / \mathrm{h}$ for 89 percent of the free-flow duration and traveled at speeds below the PSL for only 3 percent of the free-flow duration. Contrarily, the defensive drivers used speeds below the PSL for 89 percent of their free-flow duration and never exceeded the PSL by $10 \mathrm{~km} / \mathrm{h}$. For the common drivers, their speed choice was in between that of the other two driver types. 


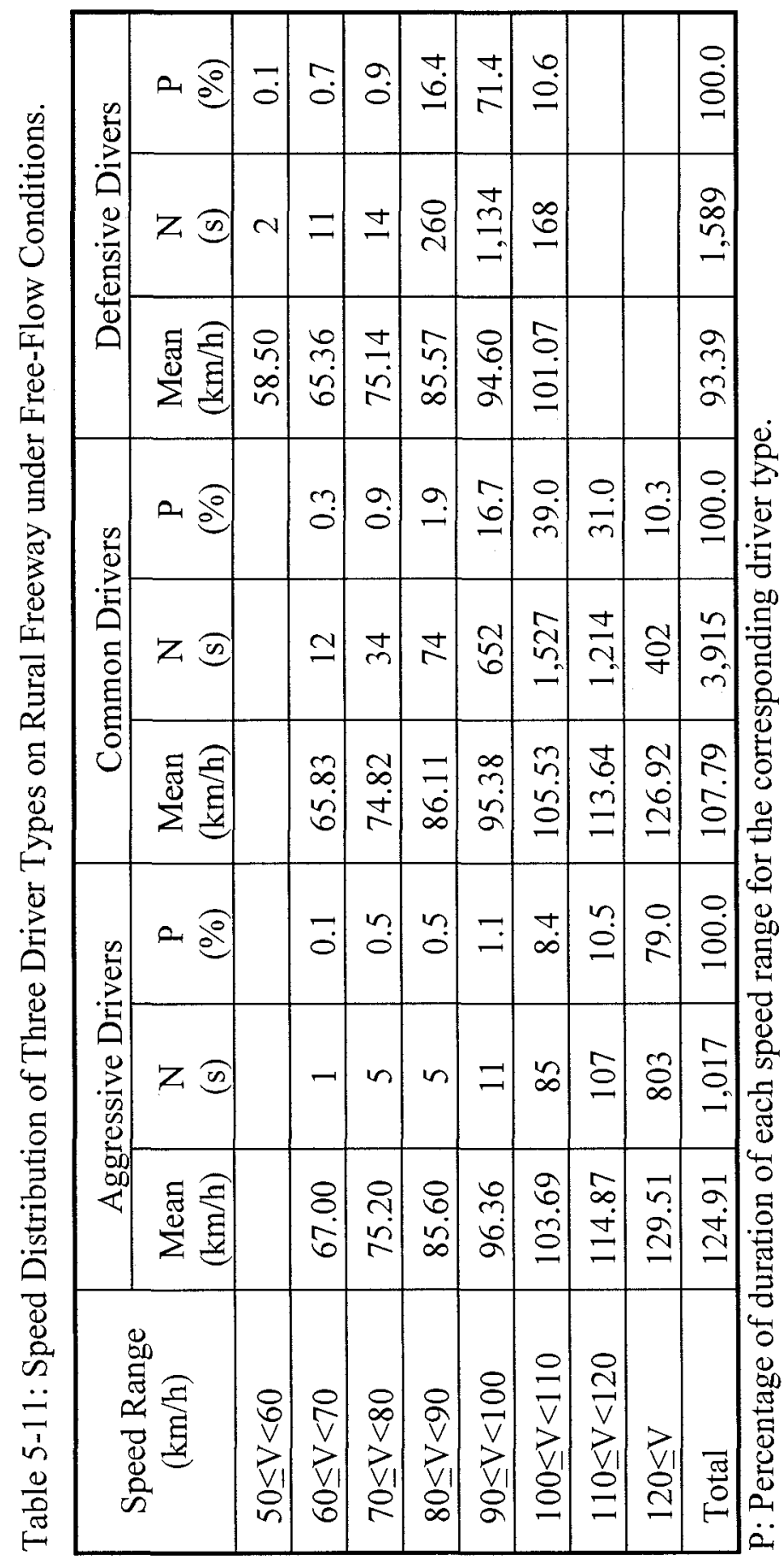




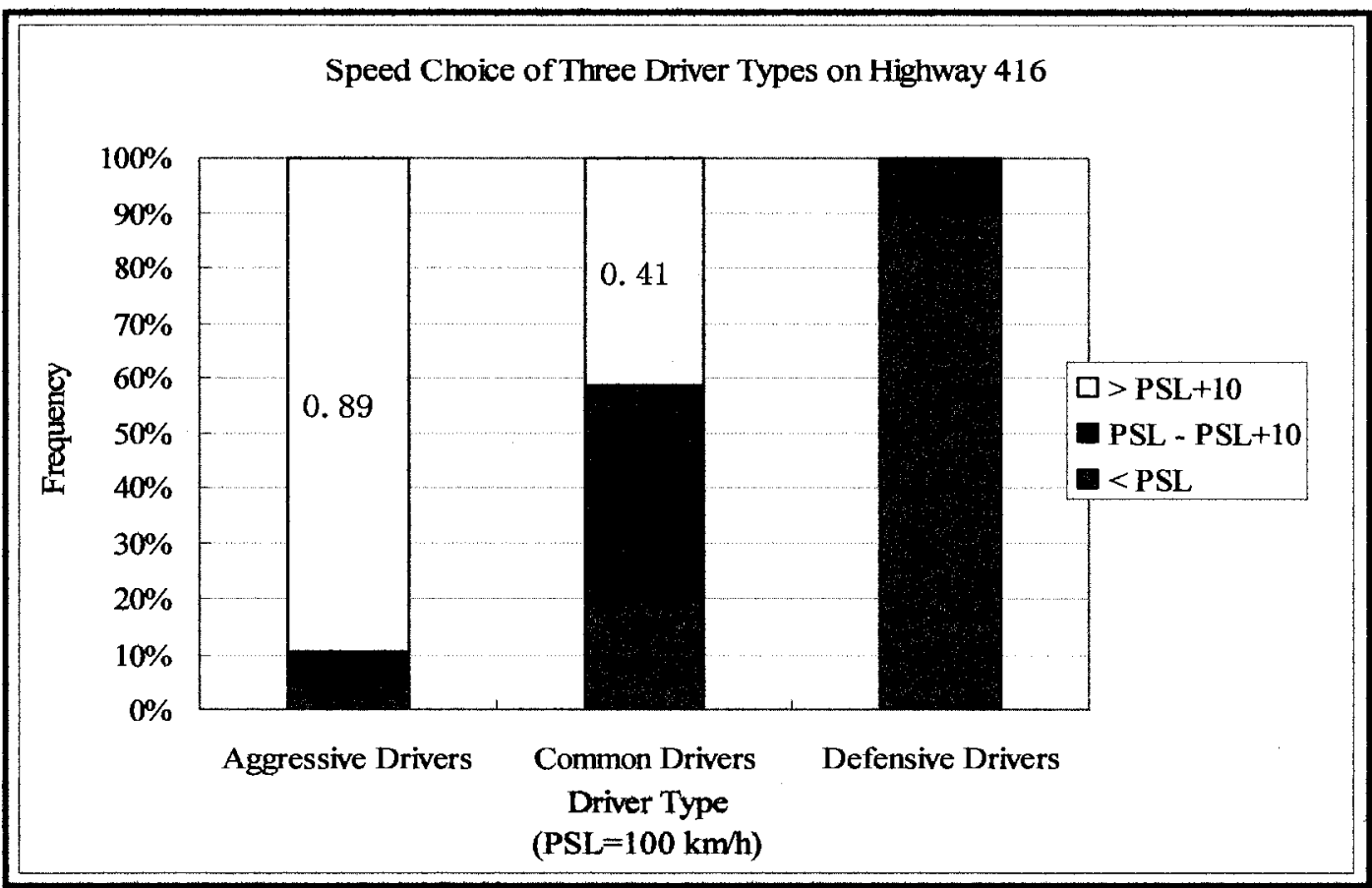

Figure 5-12: Speed Choice of Three Driver Types on Rural Freeway under Free-Flow Conditions.

\subsection{3.d Urban/Suburban Roads}

As mentioned earlier, there are three different speed limits on the urban/suburban roads in this experiment: 50,60 , and $80 \mathrm{~km} / \mathrm{h}$. Speed choices of the different driver types were analyzed in relation to the three speed limits. Again, the speed bins on the urban/suburban roads were set similar to those on the other road types. The speed distributions on urban/suburban roads for the three driver types are summarized in Tables 5-12 to 5-14, and their speed choices are depicted in Figures 5-13 to 5-15. As shown in these figures, the difference between speed choices of the three driver types on urban/suburban roads was less significant compared to that on other road classes. Nevertheless, it was still evident that aggressive drivers used larger portion of their speeds exceeding PSL over $10 \mathrm{~km} / \mathrm{h}$ than defensive drivers, especially for the case of PSL of $50 \mathrm{~km} / \mathrm{h}$. 


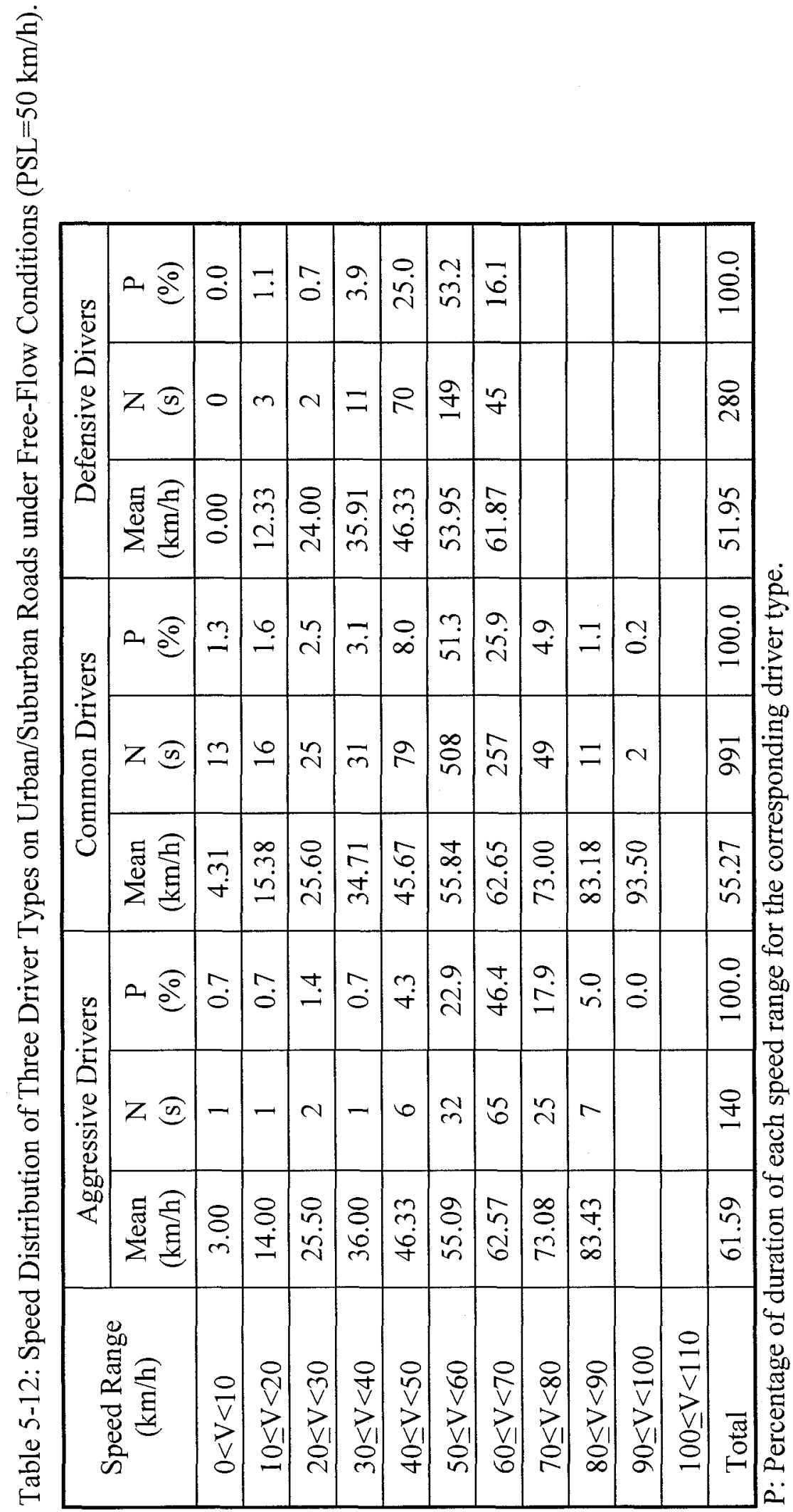




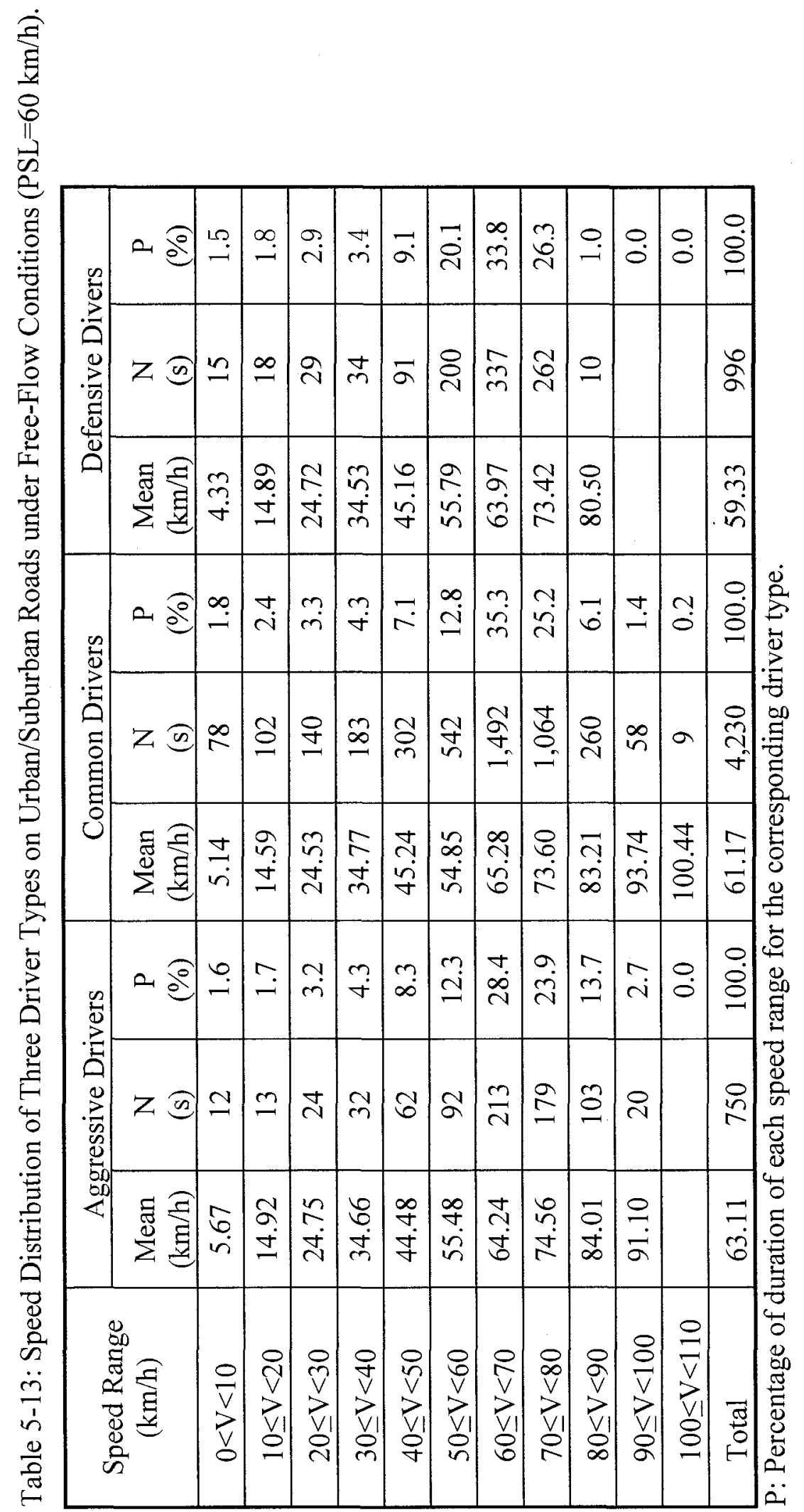




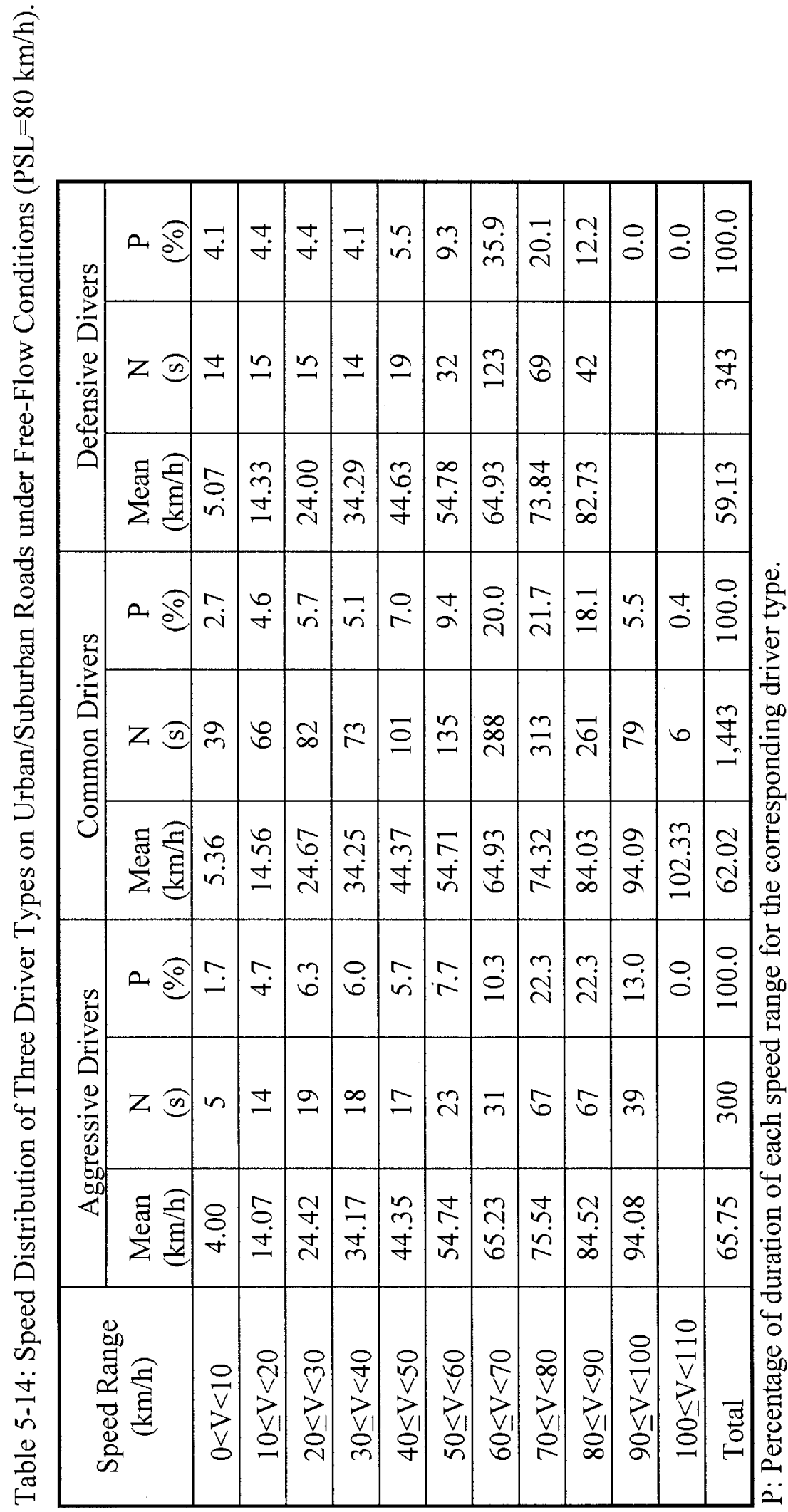




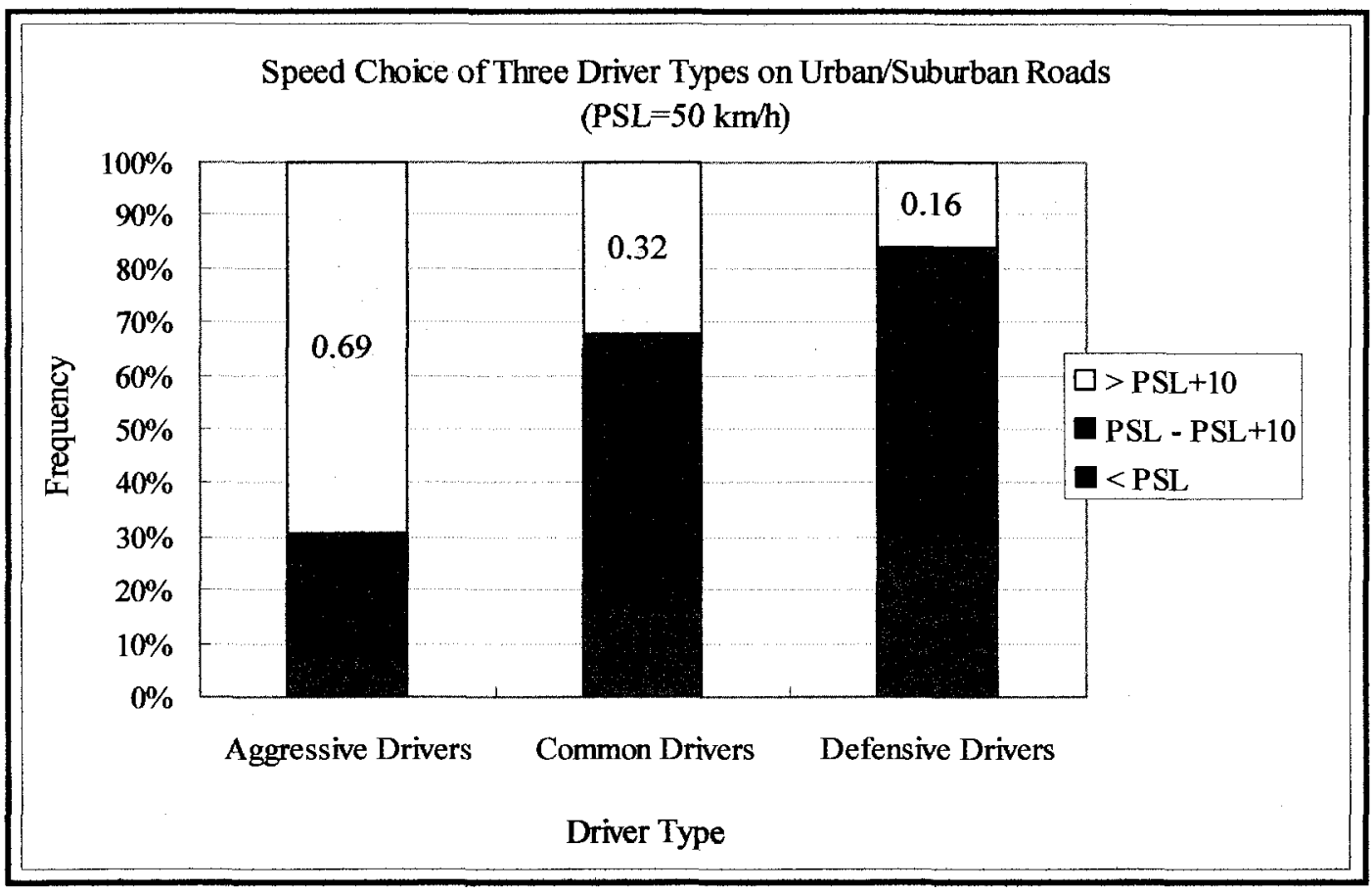

Figure 5-13: Speed Choice of Three Driver Types on Urban/Suburban Roads under FreeFlow Conditions ( $\mathrm{PSL}=50 \mathrm{~km} / \mathrm{h}$ ).

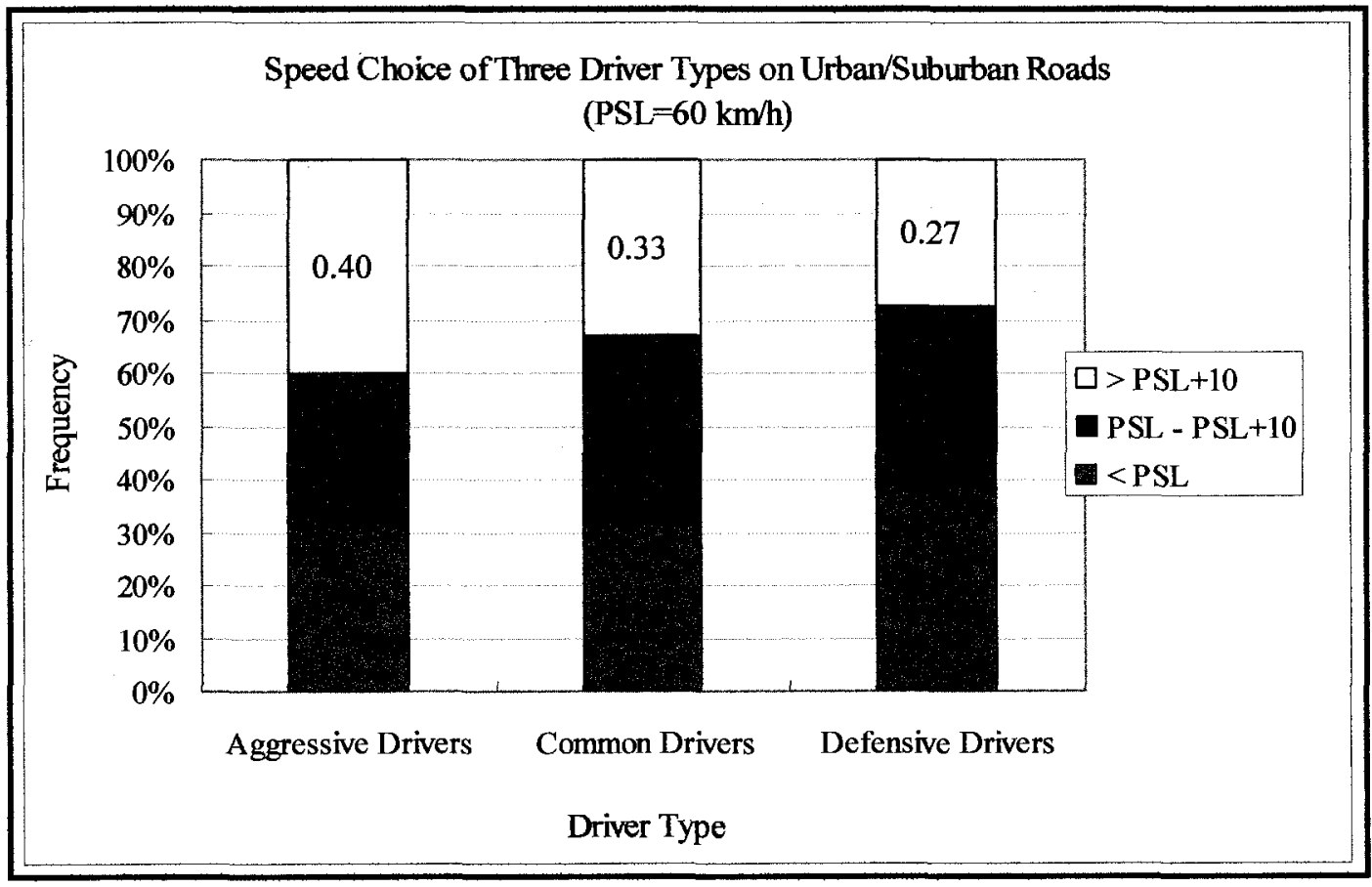

Figure 5-14: Speed Choice of Three Driver Types on Urban/Suburban Roads under FreeFlow Conditions $(\mathrm{PSL}=60 \mathrm{~km} / \mathrm{h}$ ). 


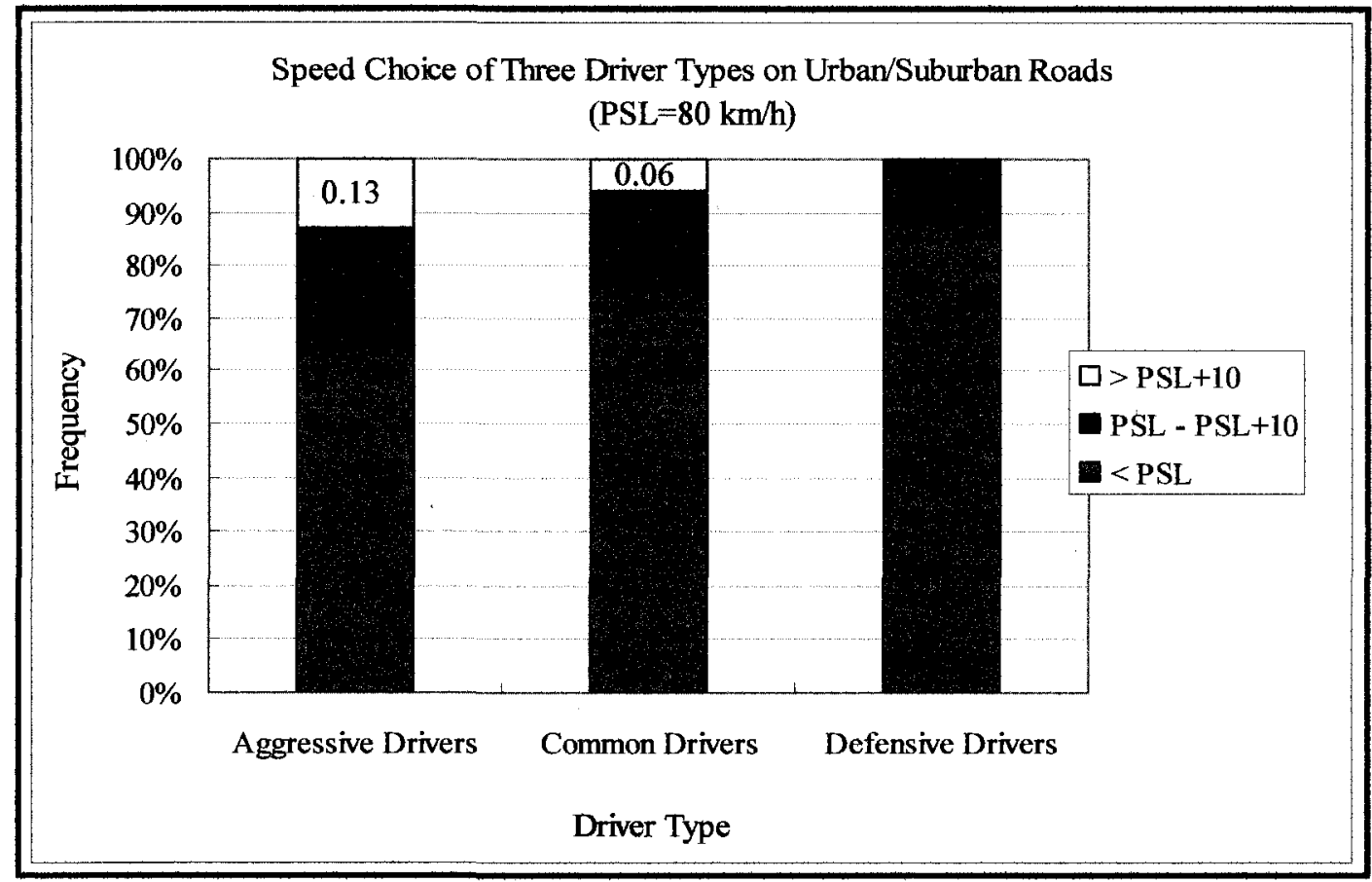

Figure 5-15: Speed Choice of Three Driver Types on Urban/Suburban Roads under FreeFlow Conditions ( $\mathrm{PSL}=80 \mathrm{~km} / \mathrm{h}$ ).

\subsection{3.e Summary of Speed Choice of the Driver Types}

Based on the analysis regarding the relationship between driver speed choice in relation to the speed limits, it is found that different types of drivers displayed distinctive characteristics in selection of their speeds when the traffic condition was favourable (i.e., free-flow conditions). The findings are summarized as follows:

- Aggressive drivers are usually willing to select high speeds. They may consider speed limits as the bottom bound.

- Defensive drivers prefer to keep their speed below the speed limits; even when the traffic condition allows for faster speeds. 
- Common drivers, who are the major portion in the driver sample, are more likely to drive at speeds not exceeding speed limits to unreasonable extents.

The findings in this research generally agree with the findings of Fitzpatrick, et al. (2003).

\subsection{Framework for Judging Driving Aggressiveness}

Another effort of this research was trying to set up a framework for judging the aggressiveness of a driver based on his/her speed behaviour on a specific trip involving roads similar to some or all of the four road types in this research. The objective of this effort is to provide a tool to tell a driver or supervisor of the driver whether he/she should change his/her driving habits. Since it is impossible to distinguish free-flow and non-freeflow speeds in a driver's daily trip if no laser gun or other device is applied to measure the distance between the vehicle and its leading vehicles, as in this experiment, only mixed flow conditions were considered in this effort.

The effort is based on several assumptions. One is that the speed choice of the 18 drivers classified as common drivers in this study is representative of the speed behaviour of common drivers in the general population. The second assumption is that the driving environment of a specific trip is not substantially different from that of this experiment. For example, the trip, upon which the driver behaviour is evaluated, should not take place in adverse weather conditions or heavy traffic congestion, which may strongly affect a driver's speed choice. The third assumption is that the driver should be under his/her normal condition during driving, i.e., without impairment of drugs or alcohol.

Judgement of the aggressiveness of a driver is made according to the relationship between the real speed distribution of the driver and the estimated speed distribution of 
common drivers making the same trip as the subject. For this, the speed distribution and its $95 \%$ confidence interval (CI) for common drivers is set up using the parameters obtained in this research. The parameters include the mean speeds of common drivers on every road class, the speed frequency in each speed range of common drivers on every road class and the standard errors in each speed range for computing the lower and upper bounds of $95 \% \mathrm{CI}$. The required variables for this prediction are the length of each road type in a trip, which can be reported by the driver.

The driver is categorized as a common driver if the percentages of each speed range in his/her data are generally within the $95 \% \mathrm{CI}$ of speed distribution of common drivers; the driver is categorized as defensive or aggressive if he/she has significant portion of speeds beyond the lower or upper bounds of $95 \%$ CI of speed distribution of common drivers. To be clearer, the procedure is introduced using an example to explain ways to identify the aggressiveness of a specific driver. Since no extra data is available, the existing data in this study are used in this example.

Table 5-15 presents the available parameters obtained in this study, of which the mean speeds of common drivers on each road class will be used for the prediction. It is supposed that the length of each road class (or the percentage of each road class in the trip) is reported. 
Table 5-15: Parameters for Judging the Aggressiveness of a Driver.

\begin{tabular}{|l|c|c|c|c|c|}
\hline & Urban & $\begin{array}{c}\text { Two-Lane } \\
\text { Rural } \\
\text { Freeway }\end{array}$ & $\begin{array}{c}\text { Rural } \\
\text { Freeway }\end{array}$ & $\begin{array}{c}\text { Urban/ } \\
\text { Suburban } \\
\text { Road }\end{array}$ & Overall \\
\hline Length $(\mathrm{km})$ & 24.9 & 51.1 & 16.5 & 15.3 & 107.8 \\
\hline $\begin{array}{l}\text { Percentage of road class } \\
\text { length }(\%)\end{array}$ & 23.1 & 47.4 & 15.3 & 14.2 & 100 \\
\hline $\begin{array}{l}\text { Percentage of travel time } \\
(\%)\end{array}$ & 18.96 & 48.36 & 11.85 & 20.83 & 100 \\
\hline $\begin{array}{l}\text { Mean speed of common } \\
\text { drivers }(\mathrm{km} / \mathrm{h})\end{array}$ & 101.48 & 81.43 & 107.49 & 56.44 & 83.12 \\
\hline $\begin{array}{l}\text { Mean speed of defensive } \\
\text { drivers }(\mathrm{km} / \mathrm{h})\end{array}$ & 94.02 & 70.44 & 93.64 & 54.12 & 74.42 \\
\hline $\begin{array}{l}\text { Mean speed of aggressive } \\
\text { drivers }(\mathrm{km} / \mathrm{h})\end{array}$ & 108.35 & 92.46 & 123.40 & 57.15 & 91.12 \\
\hline
\end{tabular}

1. The first step is estimating the mean speed of the common drivers in the whole trip.

Suppose that the total length of a trip is $L_{\text {trip }}(\mathrm{km})$, which includes the reported length for every road class: urban freeway ( $\left.L_{u r b a n-f w y}\right)$, two-lane rural highway $\left(L_{\text {2lane-hwy }}\right)$, rural freeway $\left(L_{\text {rural-fwy }}\right)$, and urban/suburban $\operatorname{road}\left(L_{u r b a n-s u b}\right)$. The known parameters of average speeds of the common drivers are 101.48, 81.43, 107.49 , and $56.44 \mathrm{~km} / \mathrm{h}$ for urban freeway, two-lane rural highway, rural freeway, and urban/suburban road, respectively. Therefore, average speed of the common drivers for the whole trip can be computed using Equation 5.1.

$$
V_{\text {trip-mean }}=\frac{L_{\text {trip }}}{\frac{L_{\text {urban-fwy }}}{101.48}+\frac{L_{2 l \text { lome-hwy }}}{81.43}+\frac{L_{\text {rural-fwy }}}{107.49}+\frac{L_{\text {urban-sub }}}{56.44}}
$$

Where $V_{\text {trip-mean }}$ is the estimated mean speed of the common drivers for the trip. Equation 5.1 can be rewritten in Equation 5.2, in which the mean speed is computed using the percentage of length for each road class in the whole trip. For 
example, $P L_{\text {urban-fwy }}$ denotes the percentage of the length of urban freeway in the trip.

$$
V_{\text {trip-mean }}=\frac{1}{\frac{P L_{\text {urban-fwy }}}{101.48}+\frac{P L_{\text {2lane-hwy }}}{81.43}+\frac{P L_{\text {rural-fwy }}}{107.49}+\frac{P L_{\text {urban-sub }}}{56.44}}
$$

Applying the equation for the test route,

$$
V_{\text {trip-mean }}=\frac{1}{\frac{0.231}{101.48}+\frac{0.474}{81.43}+\frac{0.153}{107.49}+\frac{0.142}{56.44}}=83.08 \mathrm{~km} / \mathrm{h}
$$

As shown in Table 5-15, the percentages of the length for an urban freeway, two-lane rural highways, a rural freeway and urban/suburban roads used in this computation are $23.1 \%, 47.4 \%, 15.3 \%$ and $14.2 \%$, respectively. The computed average speed $(83.08 \mathrm{~km} / \mathrm{h})$ is very close to the average speed $(83.12 \mathrm{~km} / \mathrm{h})$ produced by previous efforts.

2. The second step is to compute the percentage of time spent on each road class using Equation 5.4.

$$
\begin{aligned}
P T_{\text {road-class }} & =\frac{T_{\text {road-class }}}{T_{\text {overall }}}=\frac{L_{\text {road-class }}}{V_{\text {road-class }}} / \frac{L_{\text {trip }}}{V_{\text {trip-mean }}} \\
& =P L_{\text {road-class }} \times \frac{V_{\text {trip-mean }}}{V_{\text {road-class }}}
\end{aligned}
$$

Where $T_{\text {road-class }}$ is the travel time on this road class,

$T_{\text {overall }}$ is the total travel time in the trip,

$P T_{\text {road-class }}$ is the percentage of travel time on this road class in the total travel time,

$P L_{\text {road-class }}$ is the percentage of length of this road class in the entire trip, 
$V_{\text {trip-mean }}$ is the computed mean speed of the common drivers for the trip, which has been obtained earlier $(83.08 \mathrm{~km} / \mathrm{h})$, and $V_{\text {road-class }}$ is the known mean speed on each road class, which is 101.48 , $81.43,107.49$, and $56.44 \mathrm{~km} / \mathrm{h}$ for urban freeway, two-lane rural highway, rural freeway, and urban/suburban road, respectively.

Applying the equation for the test route,

Urban freeway: $P T_{\text {urban-fwy }}=23.1 \% \times \frac{83.08}{101.48}=18.91(\%)$

Two-lane rural highway: $P T_{\text {2lane-hwy }}=47.4 \% \times \frac{83.08}{81.43}=48.36(\%)$

Rural freeway: $P T_{\text {rural-fwy }}=15.3 \% \times \frac{83.08}{107.49}=11.83(\%)$

Urban/suburban road: $P T_{\text {urban-sub }}=14.2 \% \times \frac{83.08}{56.44}=20.90(\%)$

The computed percentages of time for each road class are almost equal to the corresponding percentages of time observed in the experiment, as shown in Table $5-15$.

3. With the known speed frequency on each road class obtained in this research, the speed distribution of the common drivers could be produced using Equation 5.5.

$$
\begin{aligned}
& F_{\text {estimated }}=P T_{\text {urban-fwy }} \times F_{\text {urban-fwy }}+P T_{2 l a n e-h w y} \times F_{2 l a n e-h w y} \\
& +P T_{\text {rural-fwy }} \times F_{\text {rural-fwy }}+P T_{u r b a n-s u b} \times F_{\text {urban-sub }}
\end{aligned}
$$

Where $F_{\text {estimated }}$ is the speed frequency in a specific speed range, $P T_{\text {urban-fwy }}, P T_{\text {2lane-hwy }}, P T_{\text {rural-fwy }}$, and $P T_{\text {urban-sub }}$ are the percentages of travel time on the corresponding road class, which were computed in the previous step, 
$F_{u r b a n-f w y}, F_{2 l a n e-h w y}, F_{\text {rural-fwy }}$ and $F_{u r b a n-s u b}$ are the speed frequencies in a specific speed range on a corresponding road class, which are the known parameters produced earlier in this research, presented in Table 5-14.

For example, as highlighted in Table 5-16, the frequency in the speed range between 50 and $60 \mathrm{~km} / \mathrm{h}$ can be computed as:

$F_{50.60}=18.91 \% \times 0.23 \%+48.36 \% \times 2.77 \%+11.83 \% \times 0+20.9 \% \times 21.54 \%=5.89 \%$

The observed speed frequency for each road class and for the whole trip and the predicted speed frequency are presented in Table 5-16. As shown in this table, the predicted speed distribution is highly close to the observed distribution. 


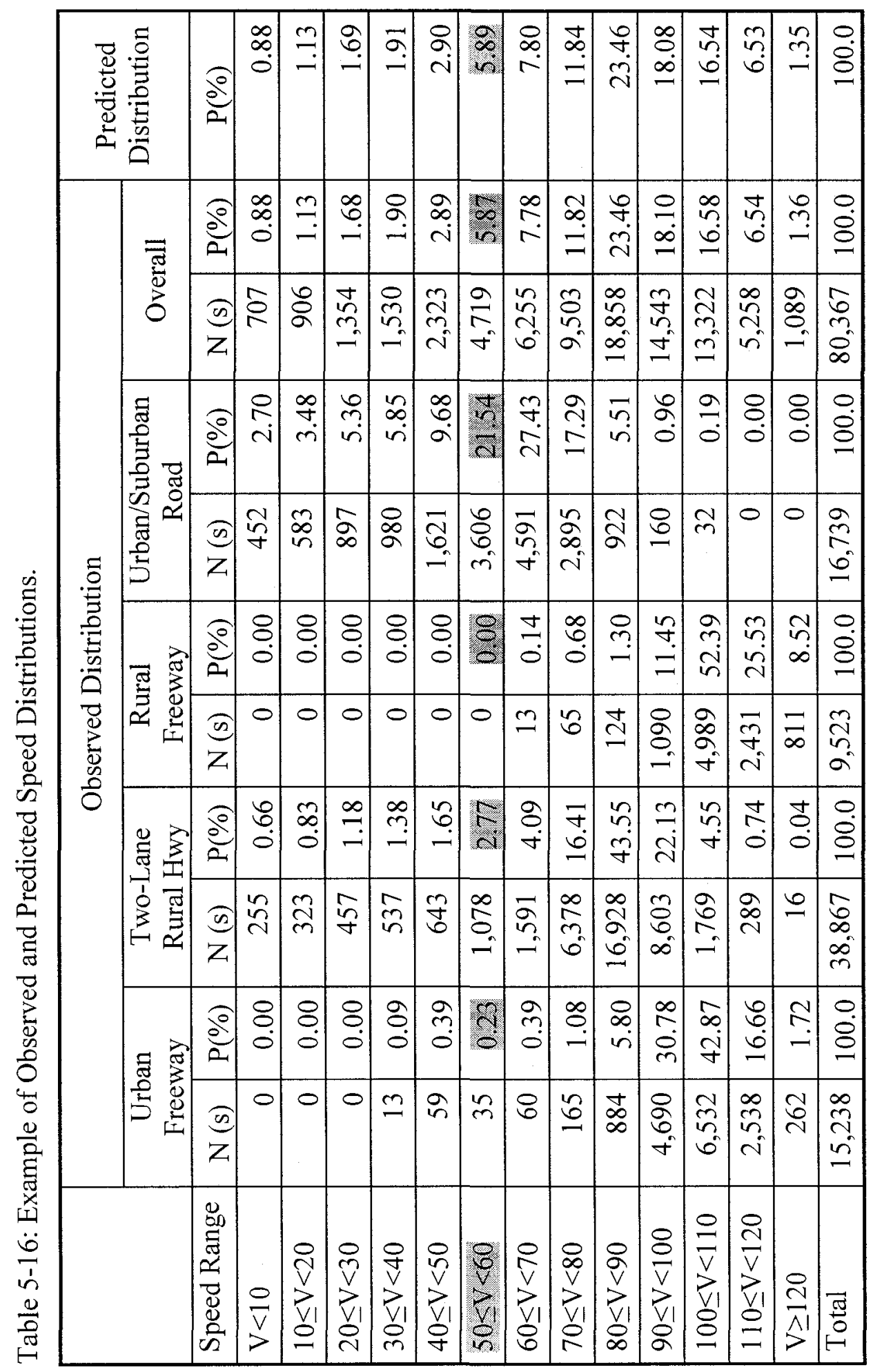


4. The next step is to set a $95 \%$ confidence interval of speed distribution for the common driver population, as shown in Table 5-17. The confidence interval is obtained using predicted speed frequency minus (lower bound) or plus (upper bound) 1.96 times standard errors, which were computed with consideration of the number of drivers in the sample.

Table 5-17: Predicted 95\% Confidence Interval of Speed Distribution of the Common Driver Population.

\begin{tabular}{|c|c|c|c|c|c|c|c|}
\hline \multirow{3}{*}{$\begin{array}{l}\text { Speed } \\
\text { Range }\end{array}$} & \multirow{2}{*}{\multicolumn{2}{|c|}{$\begin{array}{c}\text { Predicted } \\
\text { Distribution }\end{array}$}} & \multirow{3}{*}{$\begin{array}{l}\text { Std } \\
\text { Error }\end{array}$} & \multicolumn{4}{|c|}{ 95\% Confidence Interval } \\
\hline & & & & \multicolumn{2}{|c|}{ Frequency $(\%)$} & \multicolumn{2}{|c|}{$\mathrm{CF}(\%)$} \\
\hline & $\begin{array}{c}\mathrm{P} \\
(\%) \\
\end{array}$ & $\begin{array}{c}\operatorname{Cum} P \\
(\%)\end{array}$ & & Lower & Upper & Lower & Upper \\
\hline $\mathrm{V}<10$ & 0.88 & 0.88 & 0.05 & 0.77 & 0.99 & 0.77 & 0.99 \\
\hline $10 \leq \mathrm{V}<20$ & 1.13 & 2.01 & 0.05 & 1.03 & 1.23 & 1.81 & 2.22 \\
\hline $20 \leq \mathrm{V}<30$ & 1.69 & 3.70 & 0.11 & 1.48 & 1.90 & 3.29 & 4.11 \\
\hline $30 \leq \mathrm{V}<40$ & 1.91 & 5.61 & 0.08 & 1.76 & 2.06 & 5.05 & 6.17 \\
\hline $40 \leq V<50$ & 2.90 & 8.51 & 0.13 & 2.65 & 3.14 & 7.70 & 9.31 \\
\hline $50 \leq \mathrm{V}<60$ & 5.89 & 14.39 & 0.29 & 5.32 & 6.45 & 13.02 & 15.76 \\
\hline $60 \leq \mathrm{V}<70$ & 7.80 & 22.20 & 0.48 & 6.87 & 8.74 & 19.89 & 24.50 \\
\hline $70 \leq \mathrm{V}<80$ & 11.84 & 34.03 & 1.25 & 9.39 & 14.29 & 29.28 & 38.79 \\
\hline $80 \leq \mathrm{V}<90$ & 23.46 & 57.50 & 1.77 & 19.99 & 26.94 & 49.27 & 65.73 \\
\hline $90 \leq \mathrm{V}<100$ & 18.08 & 75.58 & 1.46 & 15.23 & 20.93 & 64.49 & 86.66 \\
\hline $100 \leq \mathrm{V}<110$ & 16.54 & 92.12 & 0.95 & 14.67 & 18.41 & 79.17 & $100.00^{\dagger}$ \\
\hline $110 \leq \mathrm{V}<120$ & 6.53 & 98.65 & 1.41 & 3.76 & 9.30 & 82.92 & $100.00^{\dagger}$ \\
\hline $\mathrm{V} \geq 120$ & 1.35 & 100.00 & 0.84 & $0.00^{-}$ & 3.00 & 82.63 & $100.00^{\dagger}$ \\
\hline
\end{tabular}

CF: Cumulative frequency

${ }^{\dagger}$ : Physical limit although actual calculated value is greater than 100 percent.

-: Physical limit although actual calculated value is less than 0 percent.

With the completion of these steps, the speed distribution with its $95 \%$ confidence interval of the common driver population can be produced. For summary, the parameters used for the prediction of speed distribution for any trip are emphasized, including mean speeds on each road class of the common driver sample shown in Table 5-15, observed 
speed distribution on every road class shown in Table 5-16, and standard errors for each speed range shown in Table 5-17.

With the availability of actual speed data of an examined driver, the speed distribution of this driver and the predicted speed distribution of the common drivers can be visually compared using Probability Density Function and Cumulative Distribution Function. Appendix B shows the speed distributions of individual drivers and the common driver population for both of these two functions. Driver 02,12 , and 17 are used as examples to explain the speed distribution of the common, aggressive, and defensive drivers in relation to that of the common driver population.

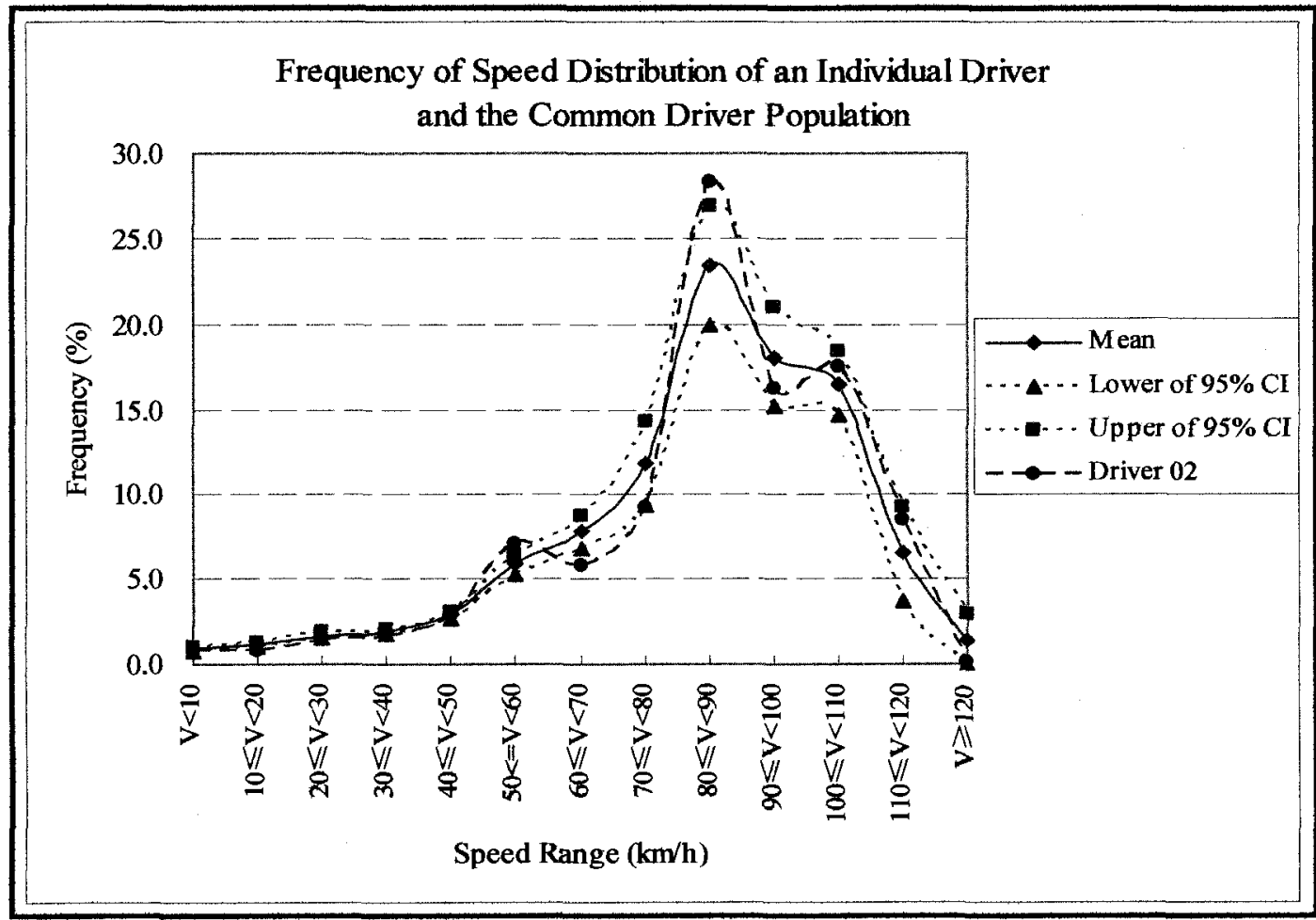

Figure 5-16: Speed Distribution of Driver 02 and the Common Driver Population. 


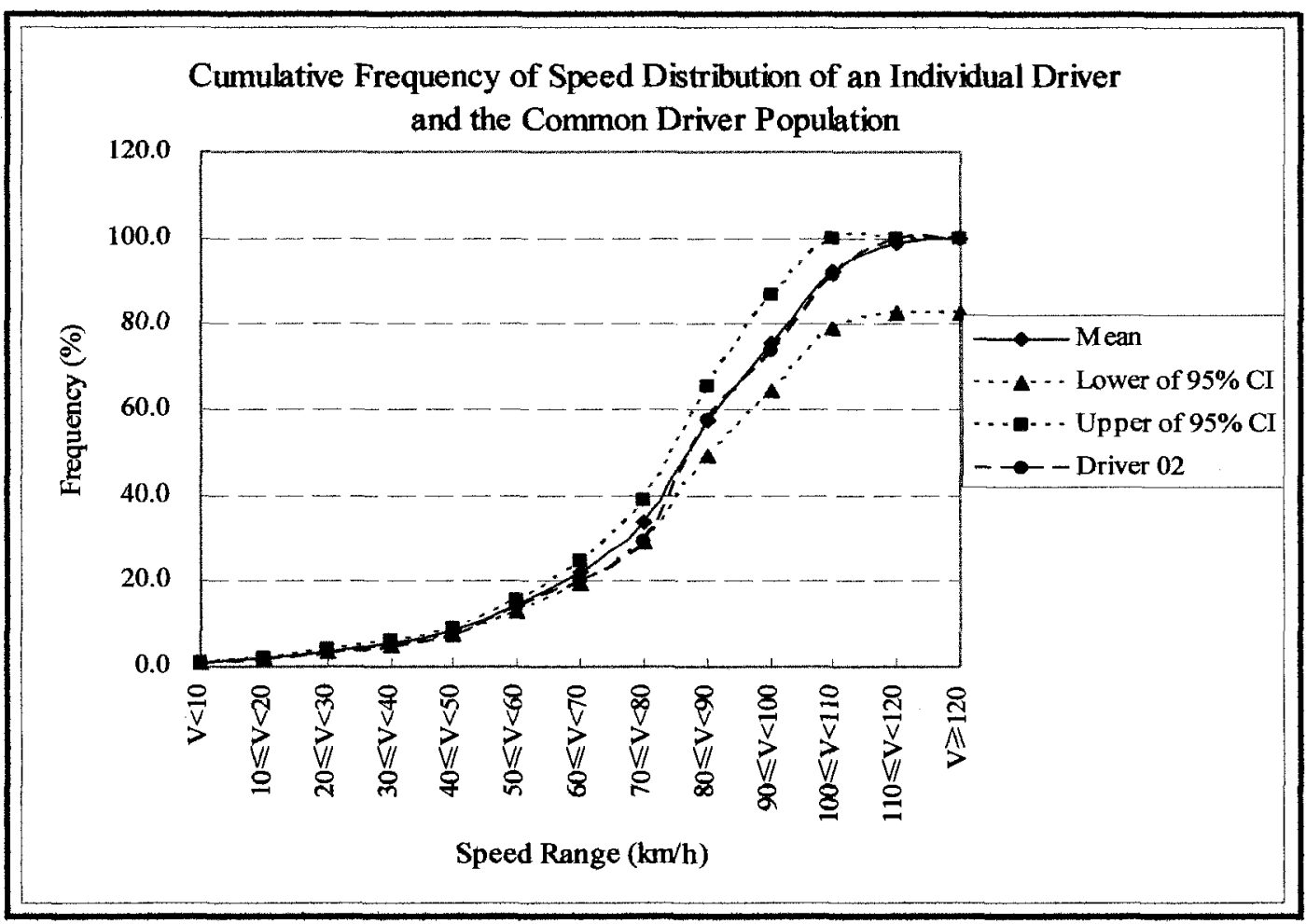

Figure 5-17: Cumulative Frequency of Speed Distribution of Driver 02 and the Common Driver Population.

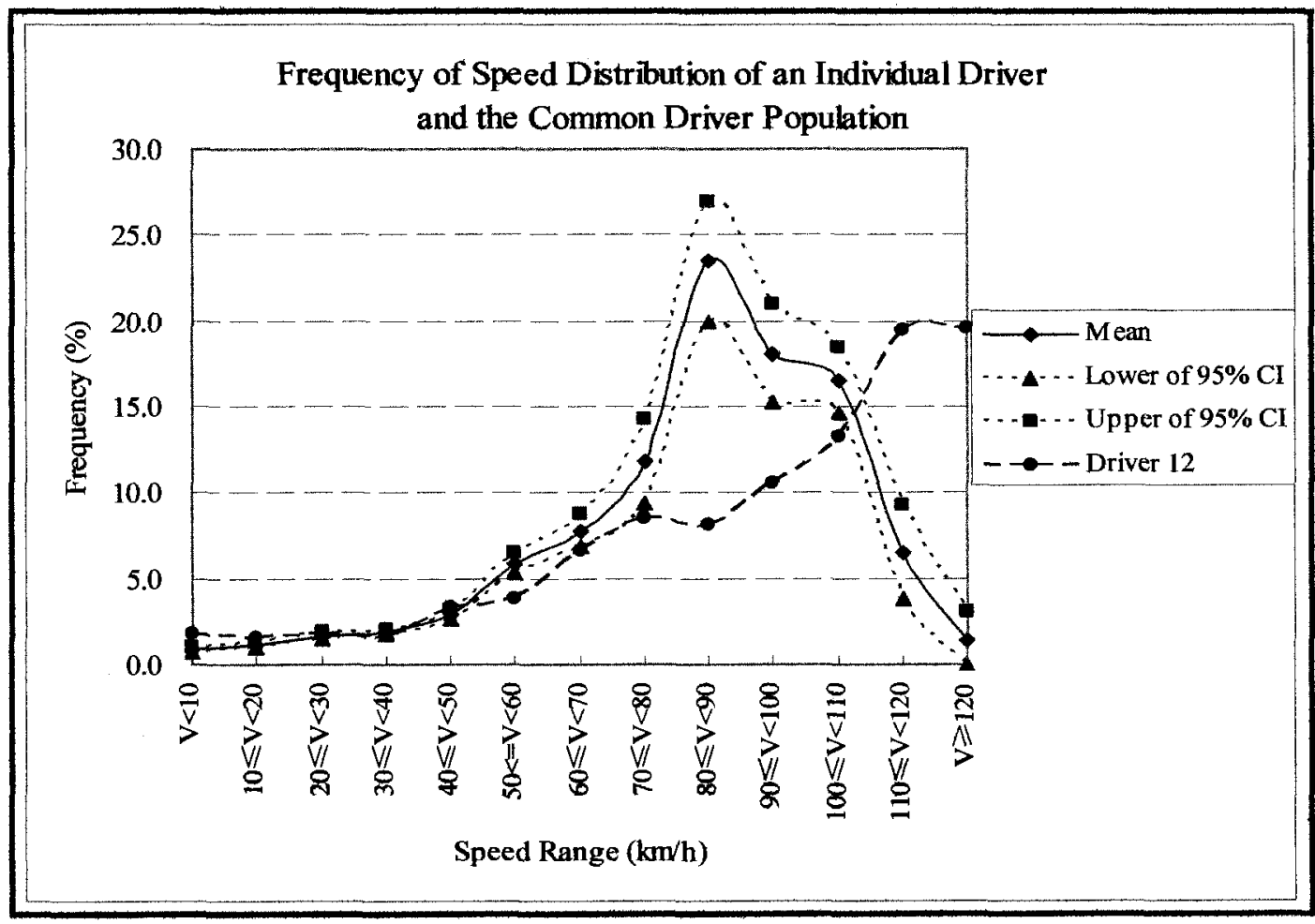

Figure 5-18: Speed Distribution of Driver 12 and the Common Driver Population. 


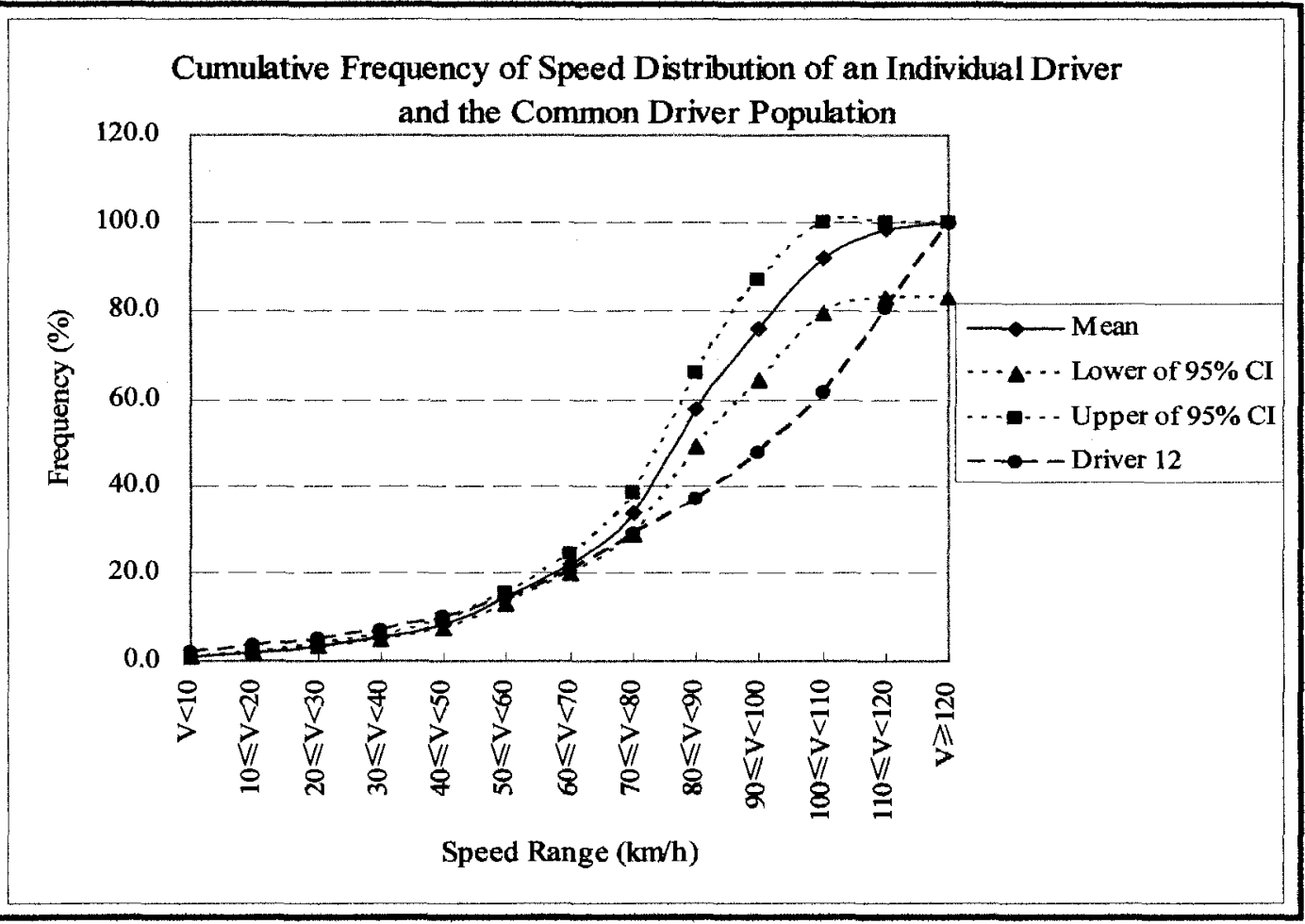

Figure 5-19: Cumulative Frequency of Speed Distribution of Driver 12 and the Common Driver Population.

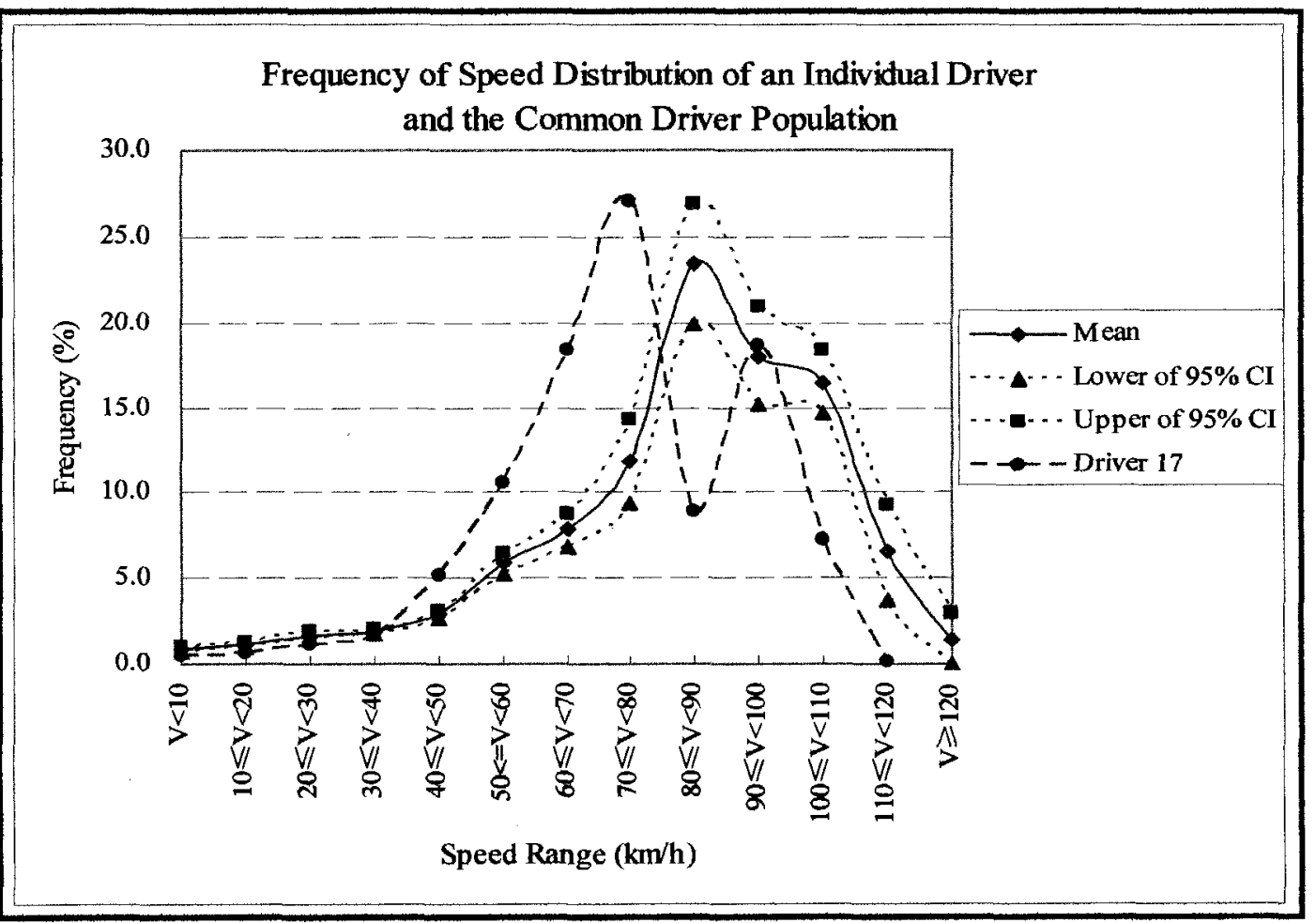

Figure 5-20: Speed Distribution of Driver 17 and the Common Driver Population. 


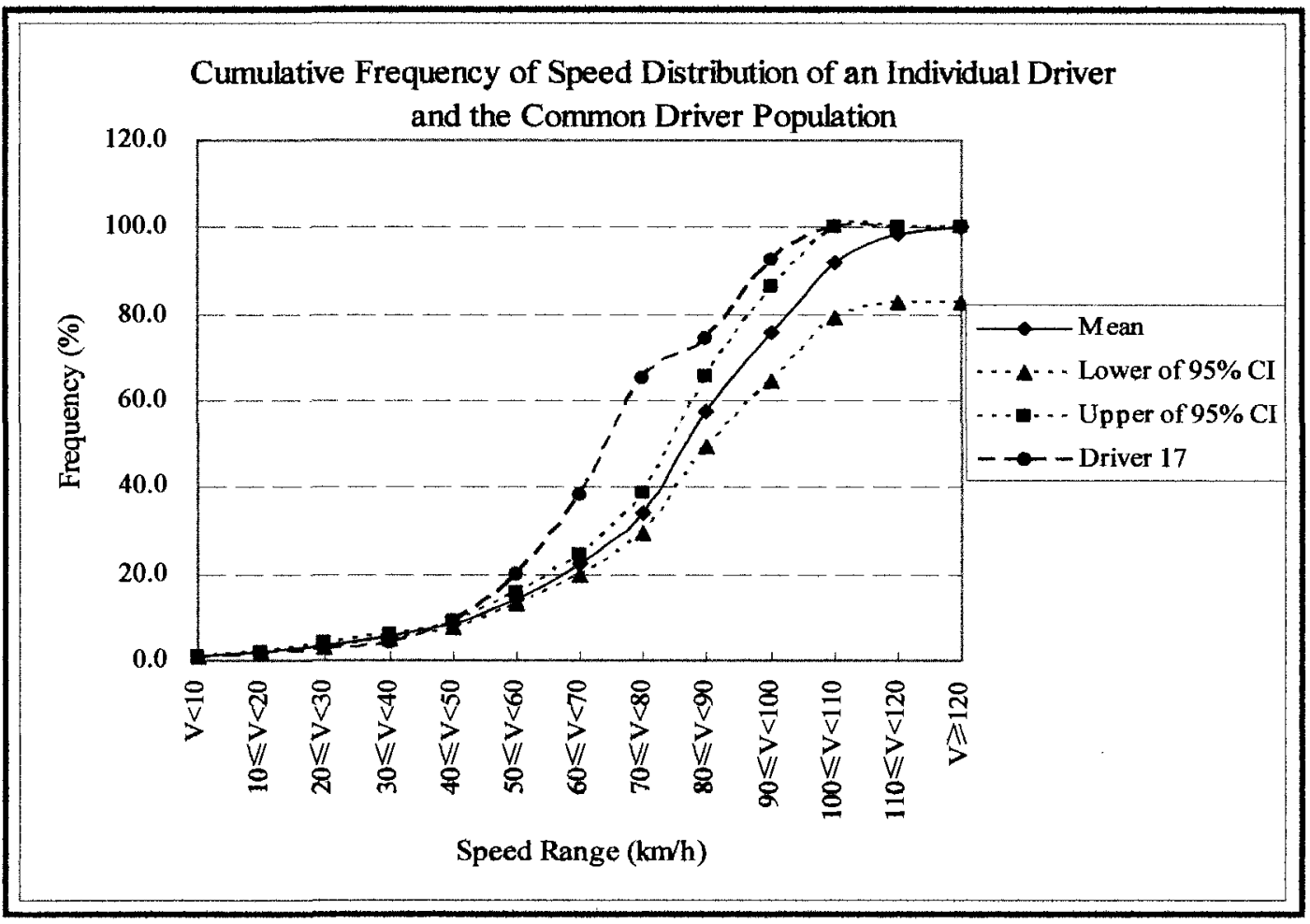

Figure 5-21: Cumulative Frequency of Speed Distribution of Driver 17 and the Common Driver Population.

According to Figures 5-16 to 5-21, the difference between speed distributions of the different types of drivers and the common driver population can be visually identified. The distribution of an individual common driver is usually within the range of $95 \% \mathrm{CI}$ of the predicted speed distribution of the common driver population. The speeds of an aggressive driver have a significant portion outside of $95 \% \mathrm{CI}$ of the predicted speed distribution of the common driver population, resulting in a larger percentage of speeds in high speed ranges. On the other hand, the speeds of a defensive driver have an obvious portion focusing on low speed ranges, percentages of which are beyond the $95 \% \mathrm{CI}$ of the speed distribution of the common driver population. Therefore, investigation of the relationship of speed distributions between an individual driver and the common driver population could be an effective approach to judge the aggressiveness of this driver. 
Moreover, comparing the mean speeds of the examined driver and the common drivers could also be used in examining the aggressiveness of this driver. In other words, an aggressive driver would have a higher mean speed than the common driver population, while a defensive driver would exhibit a lower mean speed than the driver population. As presented earlier, the average speed of the common drivers could be estimated using extracted average speeds on different road classes when the length of each road class or its percentage in a trip is given.

Nevertheless, this method for judging the aggressiveness of a driver should be used with a certain degree of caution due to a number of limitations. One limitation is that the driver sample in this study is relatively small, which may produce a result with certain bias. For example, Driver 18, who was categorized as a common driver because he showed only three aggressive driving manners out of the eight categories as explained in 5.3.1 in this chapter, had a speed distribution that exhibits the driving trend of aggressive drivers if compared with the speed distribution of the common driver population. This limitation is expected to be overcome if a lager volume of data from the Netistix pilot project is used in upgrading this framework. The second limitation is that the applicability of this method may be weak if only urban/suburban roads are involved in a specific trip, because the difference of speed behaviours between different driver types is not so evident in a driving environment with frequent stops and heavy traffic, as discussed earlier. Another limitation is that this method is only set for general driving condition. However, many various factors, such as peak-hour or off-peak hour, traffic composition, pavement condition, etc., strongly affect a driver's speed behaviour. 


\section{CHAPTER 6: DRIVER ACCELERATION AND DECELERATION BEHAVIOUR}

This chapter presents the analysis of driver acceleration behaviour corresponding to each road type under investigation; the road types are urban freeway, two-lane rural highway, rural freeway and urban/suburban road. This chapter also includes the analysis and comparison of acceleration behaviour of the three driver types that were identified based on their speed behaviour as explained in the previous chapter. Generally, a driver's instantaneous speed may fall in one of three modes: acceleration, deceleration, and cruise. Acceleration mode indicates the cases in which the vehicle's instantaneous acceleration rates are positive, whereas the deceleration mode indicates the cases in which the vehicle's instantaneous acceleration rates are negative and cruise mode denotes the cases in which the vehicle's instantaneous acceleration rates are zero. The three operation modes can be determined by the acceleration rates that are computed using the instantaneous speeds recorded by the VIU. The term acceleration here is frequently used to cover the three operation modes of acceleration, deceleration, and cruise.

\subsection{Computation of Instantaneous Acceleration Rates}

The vehicle's instantaneous acceleration rates were computed using the instantaneous speeds recorded by the VIU in the experiment. A central-difference approach was used for the computation, as shown in Equation 6.1, which shows that the vehicle's acceleration rate at time $t$ is determined by the difference between the speeds at previous and successive instants of time $t$. 


$$
a_{t}=\frac{v_{t+\Delta t 1}-v_{t-\Delta t 2}}{\Delta t 1+\Delta t 2}
$$

Where $a_{t}=$ Vehicle's instantaneous acceleration rate at time $t\left(\mathrm{~m} / \mathrm{s}^{2}\right)$, $\Delta t 1=$ Time interval between observations at time $t$ and next instant $(t+\Delta t 1)$ $(\mathrm{s})$ $\Delta t 2=$ Time interval between observations at time $t$ and prior instant $(t-\Delta t 2)$ $(\mathrm{s})$,

$v_{t+\Delta t 1}=$ Vehicle's instantaneous speed at time $t+\Delta t l(\mathrm{~m} / \mathrm{s})$, and $v_{t-\Delta t 2}=$ Vehicle's instantaneous speed at time $t-\Delta t 2(\mathrm{~m} / \mathrm{s})$.

The time intervals, $\Delta t 1$ and $\Delta t 2$, could be different. An example of calculated instantaneous acceleration rates in the database is shown in Column AF in Figure 6-1.

Alternatively, two other approaches, the forward-difference approach and the backward-difference approach, can be used for computation of acceleration rates. The forward-difference approach uses the observed speeds at time $t$ and the successive instant, while the backward-difference approach uses the observed speeds at time $t$ and the prior instant. Central-difference schemes have been shown to provide the smallest estimation errors (Burden and Faires, 1997). However, the instantaneous acceleration rate of the first observation could be only computed using the forward-difference approach, as shown in Equation 6.2; and the instantaneous acceleration rate of the last observation was computed using the backward-difference approach, as shown in Equation 6.3. In both equations, the time difference between speeds $v_{2}$ and $v_{l}$, and between speed $v_{n}$ and $v_{n-l}$ is one second, speed is in units of $\mathrm{km} / \mathrm{h}$, and acceleration rate is in units of $\mathrm{m} / \mathrm{s}^{2}$.

$$
a_{l}=\left(v_{2}-v_{l}\right) / 3.6
$$




$$
a_{n}=\left(v_{n}-v_{n-1}\right) / 3.6
$$

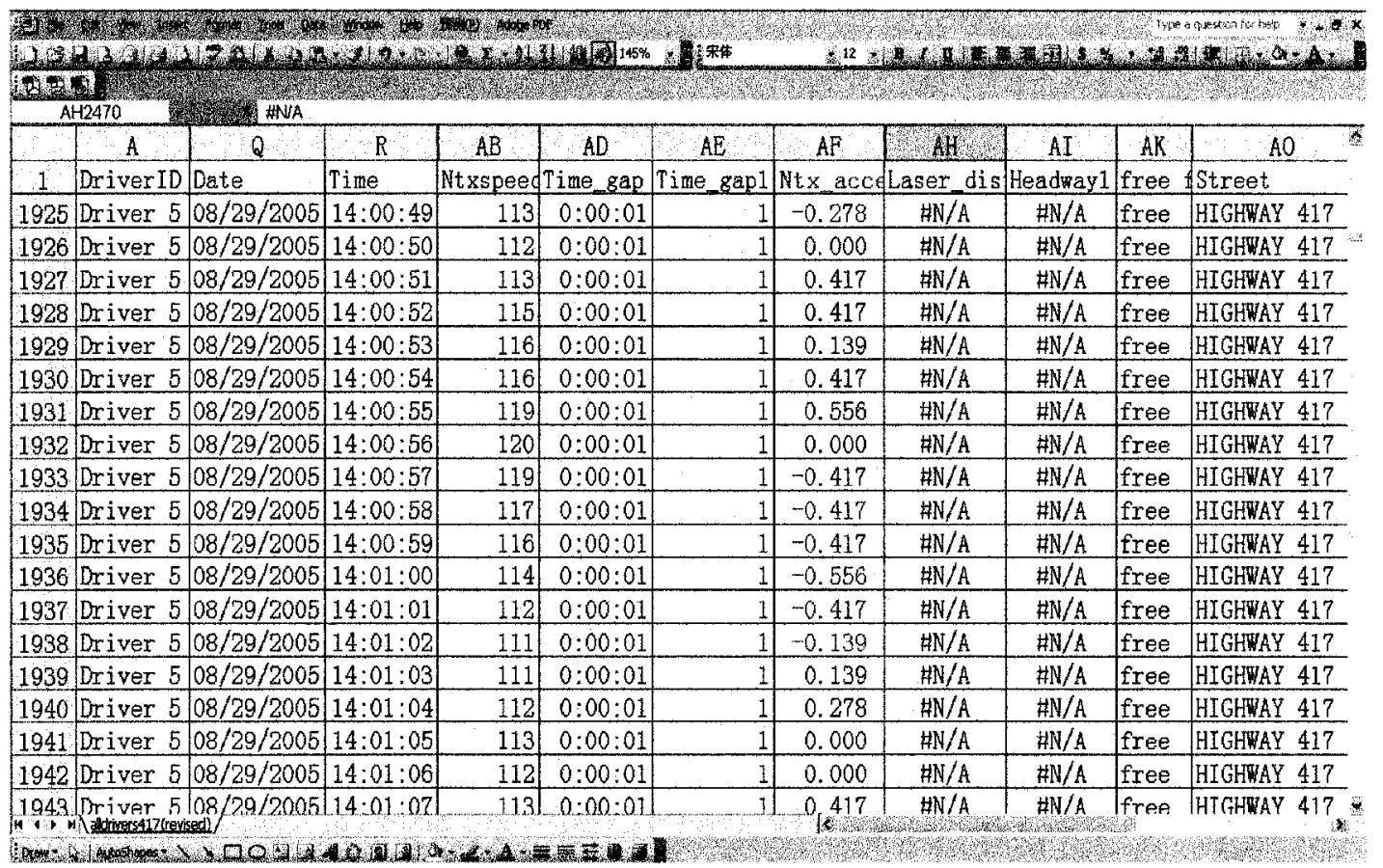

Figure 6-1: Example of Calculated Acceleration Rates in the Database.

According to the sign of instantaneous rates, a new dummy variable was introduced to express the three operation modes in SPSS v15.0. As mentioned earlier, drivers in this study were sorted into three types according to their aggressiveness in speed choice, and the speeds were also marked as free-flow and non-free-flow. Thus, the analyses of driver acceleration and deceleration behaviour were conducted for different traffic flow conditions corresponding to the four road classes and the three driver types.

\subsection{Driver Acceleration/Deceleration Behaviour on Different Road Classes}

The acceleration/deceleration behaviour of the driver sample under mixed-flow conditions, free-flow conditions and non-free-flow conditions was analyzed for each individual road class. Moreover, the acceleration/deceleration behaviour of the driver sample under free-flow conditions was also compared for the different road classes to find out if different road environments have an impact on driver behaviour in using their 
throttle or braking. In the analyses, the acceleration and deceleration were examined in relation to different speed ranges since it is believed that driver acceleration and deceleration performances are strongly related to vehicle speeds. As a result, the mean acceleration rates were illustrated in a speed-acceleration profile in order to provide a better, visual understanding of driver acceleration performance. These analysis results are discussed in detail in the following section under each road class, followed by a comparison of acceleration behaviour across different road classes.

\subsubsection{Urban Freeway (Highway 417)}

Table 6-1 shows the statistics of acceleration rates on Highway 417 and Figure 6-2 illustrates the average acceleration rates associated with driving speeds for the three flow conditions on Highway 417. According to Table 6-1 and Figure 6-2, one can see that the acceleration rates decreased, on average, with the increase of driving speed in the speed ranges between 60 and $110 \mathrm{~km} / \mathrm{h}$ under either free-flow or non-free-flow conditions. However, the trend of acceleration use in low speed ranges (i.e., speeds less than $60 \mathrm{~km} / \mathrm{h}$ ) was contrary to that in the speeds ranges of 60 to $110 \mathrm{~km} / \mathrm{h}$. This indicates that the drivers had few chances to use hard acceleration to speed up when traffic was heavy. On the other hand, drivers would accelerate quickly in a short time to their desired speeds when the traffic condition changed to favourable, as indicated by the much higher acceleration rates in the speed ranges of 60 to $80 \mathrm{~km} / \mathrm{h}$ under free-flow conditions. When vehicle speeds reached high speed ranges (i.e., speeds higher than $80 \mathrm{~km} / \mathrm{h}$ ), drivers preferred to use their throttle in a gentle manner and driver acceleration behaviour under free-flow and non-free-flow conditions had no obvious difference on average. For instance, the average acceleration rates were around $0.2 \mathrm{~m} / \mathrm{s}^{2}$ for both free-flow and non-free-flow conditions when vehicle speeds exceeded $80 \mathrm{~km} / \mathrm{h}$. 


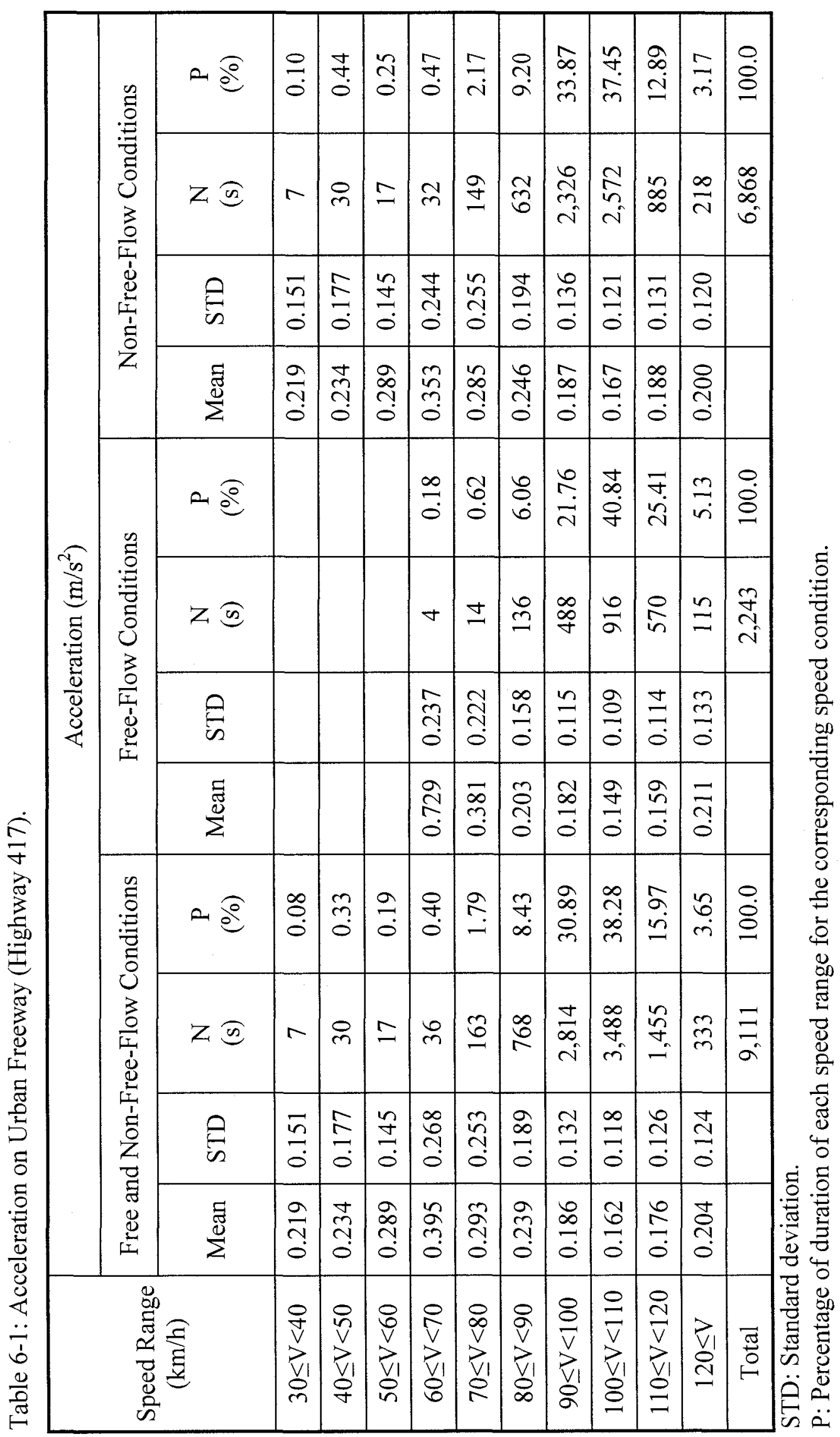




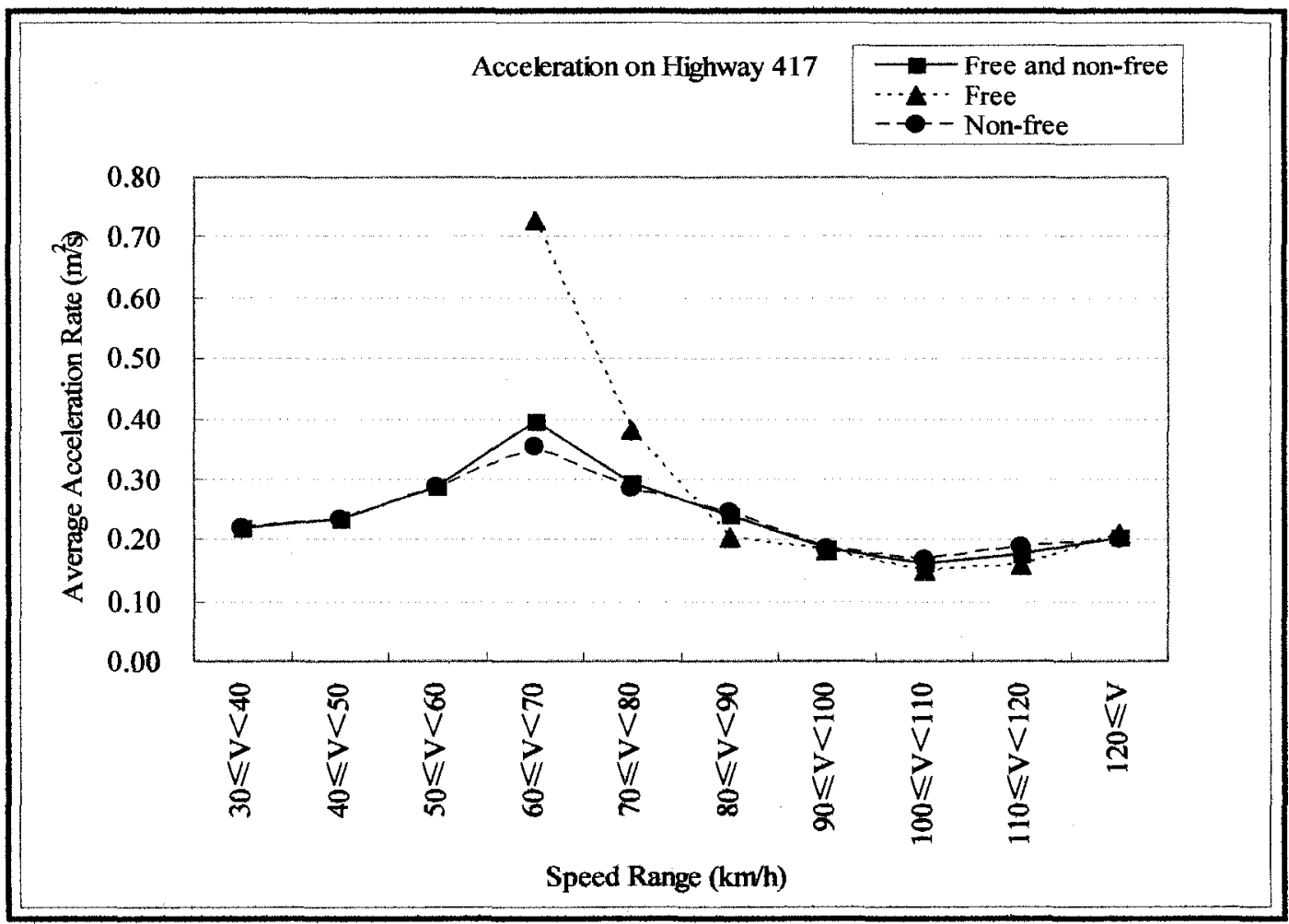

Figure 6-2: Acceleration on Urban Freeway (Highway 417).

Table 6-2 presents the statistics of deceleration rates on Highway 417 and Figure 6-3 depicts the average deceleration rates in each speed range on this road. As shown in the table and figure, the average deceleration rates fluctuated between different speed ranges for non-free-flow conditions, and they were generally higher than those for the free-flow conditions in the same speed ranges. The fluctuation implies that the drivers had to change their deceleration more often when the vehicle interacted with surrounding vehicles under non-free-flow conditions, and the higher deceleration under non-free-flow conditions indicates that a harder deceleration would be applied when the vehicle was constrained. However, the average decelerations rates on the freeway were relatively low as shown in the table and figure. 


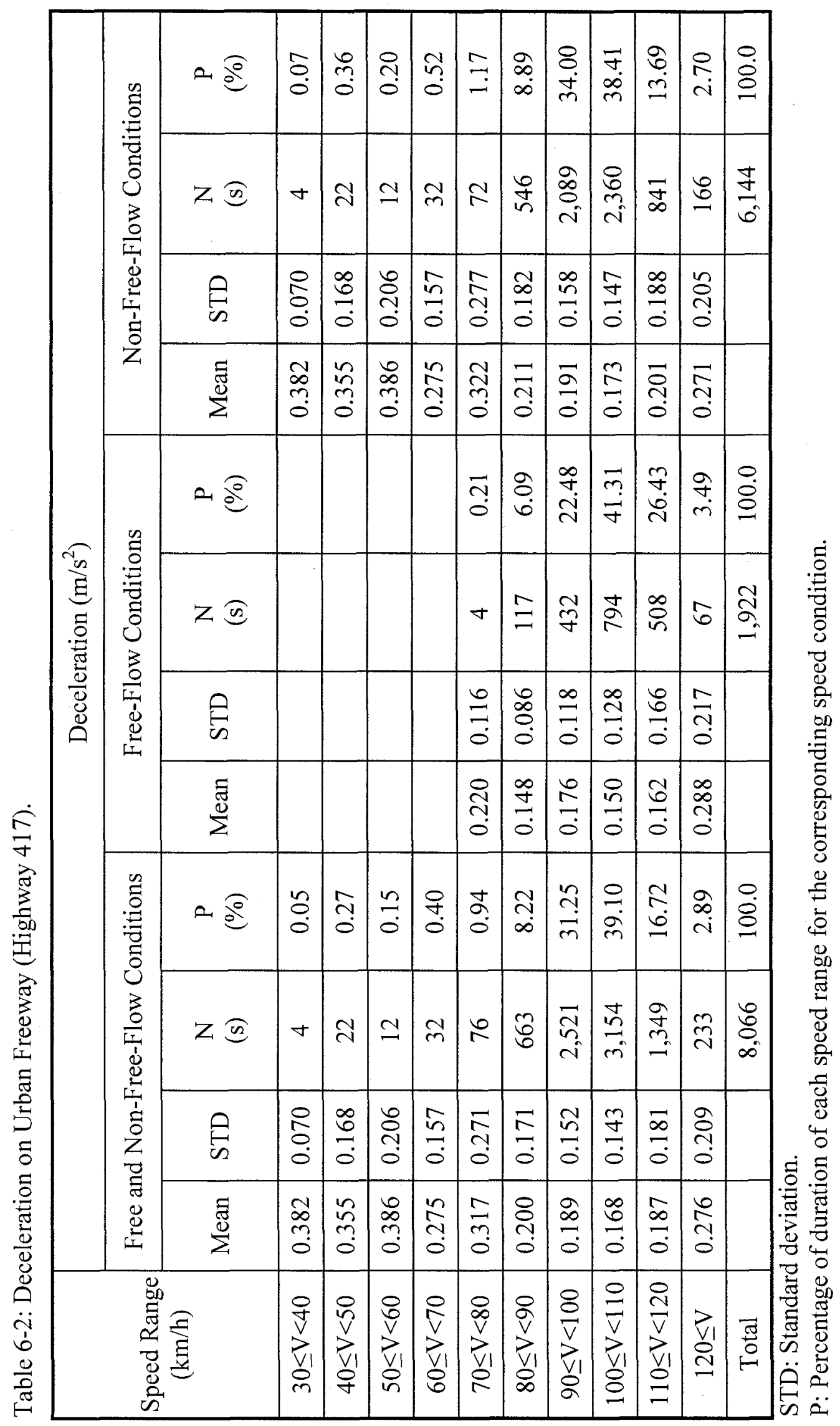

9 


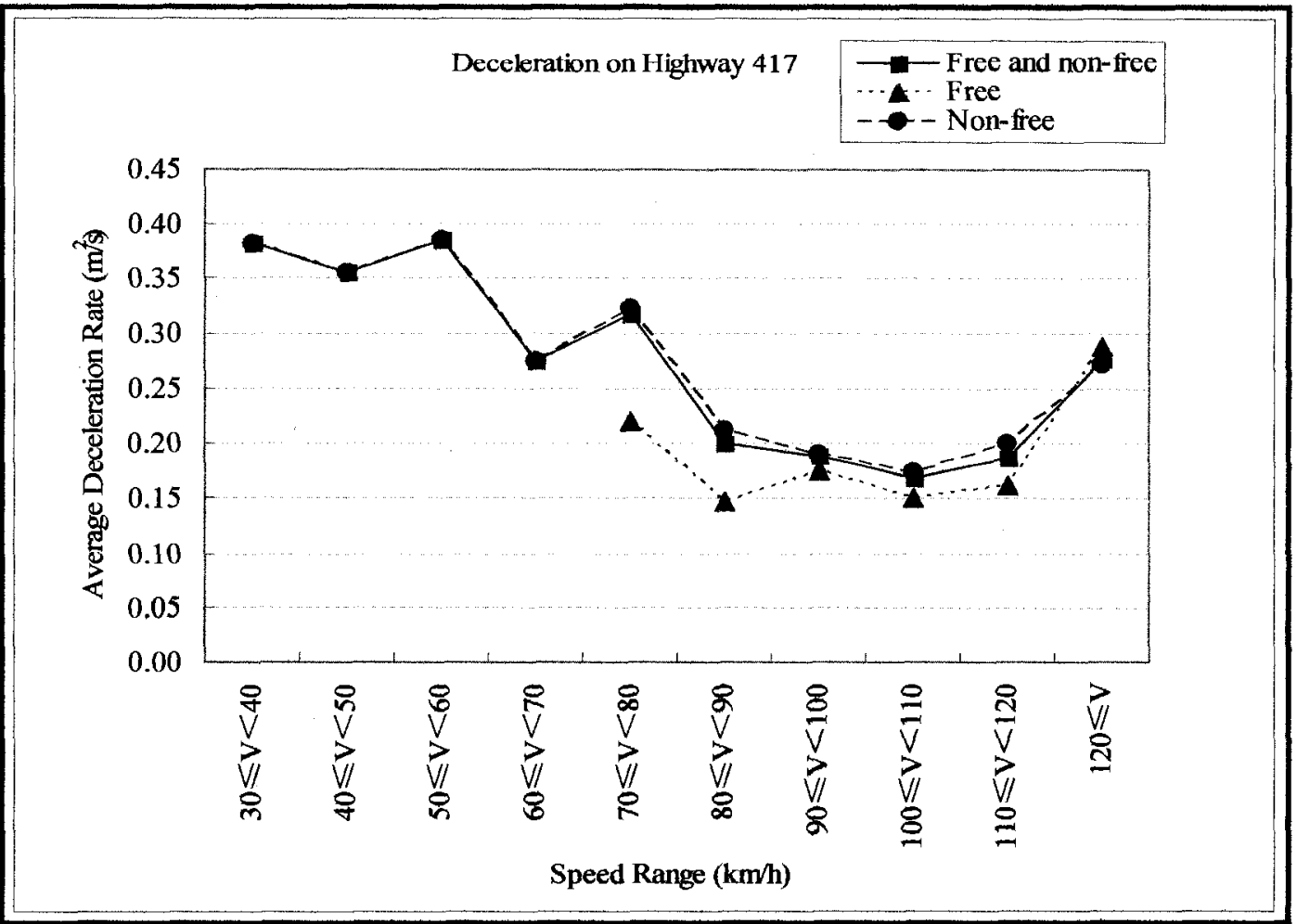

Figure 6-3: Deceleration on Urban Freeway (Highway 417).

\subsubsection{Two-Lane Rural Highways}

Table 6-3 shows the statistics of acceleration rates on two-lane rural highways and Figure 6-4 illustrates the average acceleration rates associated with driving speeds for the three flow conditions on these roads. Table 6-4 and Figure 6-5 show the statistics and illustrate the deceleration performance on two-lane rural highways.

As shown in Figure 6-4, the acceleration use of the driver sample on two-lane rural highways had two distinct differences, one was the trend of decreasing acceleration rates with the increase of speed when speeds were lower than $80 \mathrm{~km} / \mathrm{h}$ and the other was the relatively stable state of acceleration when speeds exceeded $80 \mathrm{~km} / \mathrm{h}$. However, the highest average acceleration was not associated with the lowest speed range, which 
corresponded to acceleration after a complete stop. Rather the highest acceleration rate corresponded to the speed range of 10 to $20 \mathrm{~km} / \mathrm{h}$ after the vehicle had gained some speed.

Figure 6-4 also clearly shows that the average acceleration rates under free-flow conditions were higher than those under non-free-flow conditions in low and moderate speed ranges (i.e., speeds lower than $60 \mathrm{~km} / \mathrm{h}$ ). This indicates that the drivers had more chances to accelerate their vehicle quickly to appropriate speeds when the traffic conditions were favourable and they would do so. However, they were less likely to use high acceleration when their vehicle was constrained. Moreover, the average acceleration rates for free-flow and non-free-flow conditions tended to get closer to each other in the same speed range when the speeds were high enough (i.e., speeds greater then $60 \mathrm{~km} / \mathrm{h}$ ), and they became stable when the speeds exceeded $70 \mathrm{~km} / \mathrm{h}$. This observation implies that the acceleration behaviour of the driver sample showed no big difference in the high speed ranges, and that the drivers would try to keep their vehicle traveling at a stable rate in the high speed ranges in accordance with the traffic and highway conditions if they had no reason not to do so. The average acceleration rates in the stable state were around 0.2 $\mathrm{m} / \mathrm{s}^{2}$ in the high speed ranges (i.e., speeds greater than $70 \mathrm{~km} / \mathrm{h}$ ). 


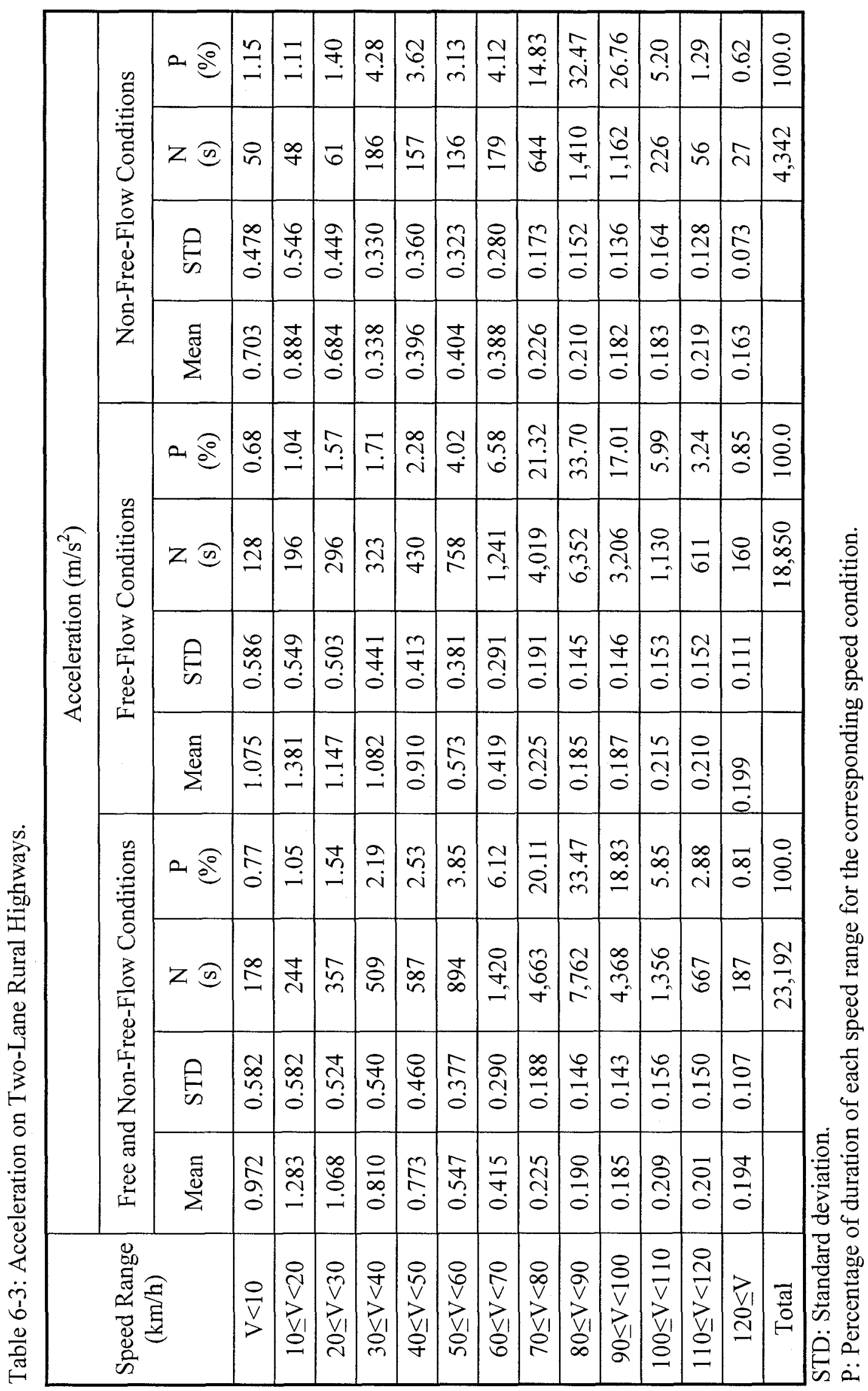




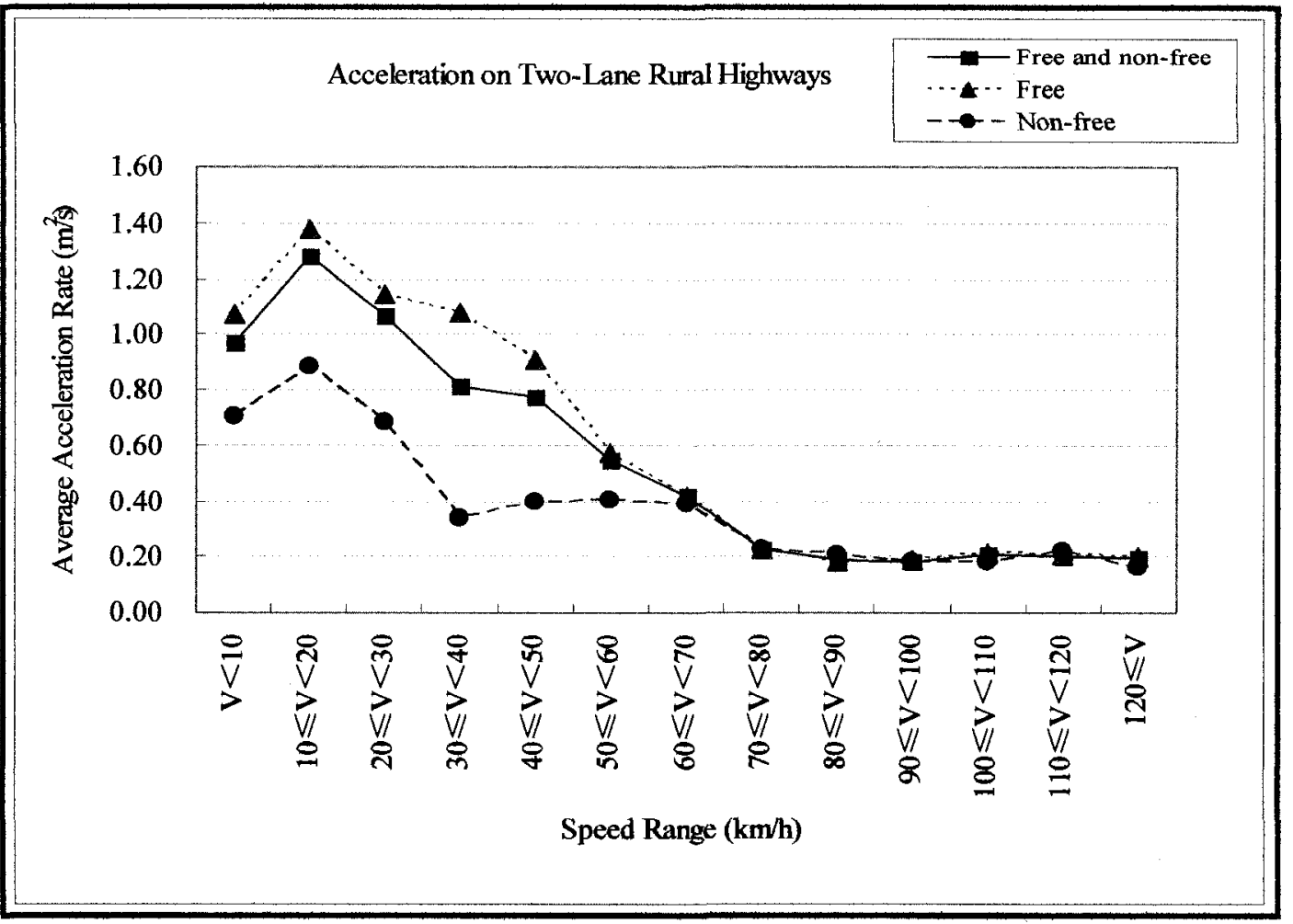

Figure 6-4: Acceleration on Two-Lane Rural Highways

As shown in Figure 6-5, the deceleration behaviour of the driver sample in general exhibited similar trends to the acceleration behaviour. Namely, the average deceleration generally decreased with the increase of speed when speeds were less than $80 \mathrm{~km} / \mathrm{h}$, but the highest average rate did not occur in the bottom speed range that represented the moment before a complete stop. Rather the highest deceleration took place in the speed ranges of 10 to $20 \mathrm{~km} / \mathrm{h}$. The average deceleration tended to be stable when speeds were higher than $80 \mathrm{~km} / \mathrm{h}$. The figure also displays evident differences of average deceleration between free-flow and non-free-flow conditions when speeds were less than $50 \mathrm{~km} / \mathrm{h}$. On the other hand, the average deceleration rates under the two flow conditions became close to each other for the same speed range when the speeds were above $50 \mathrm{~km} / \mathrm{h}$. 


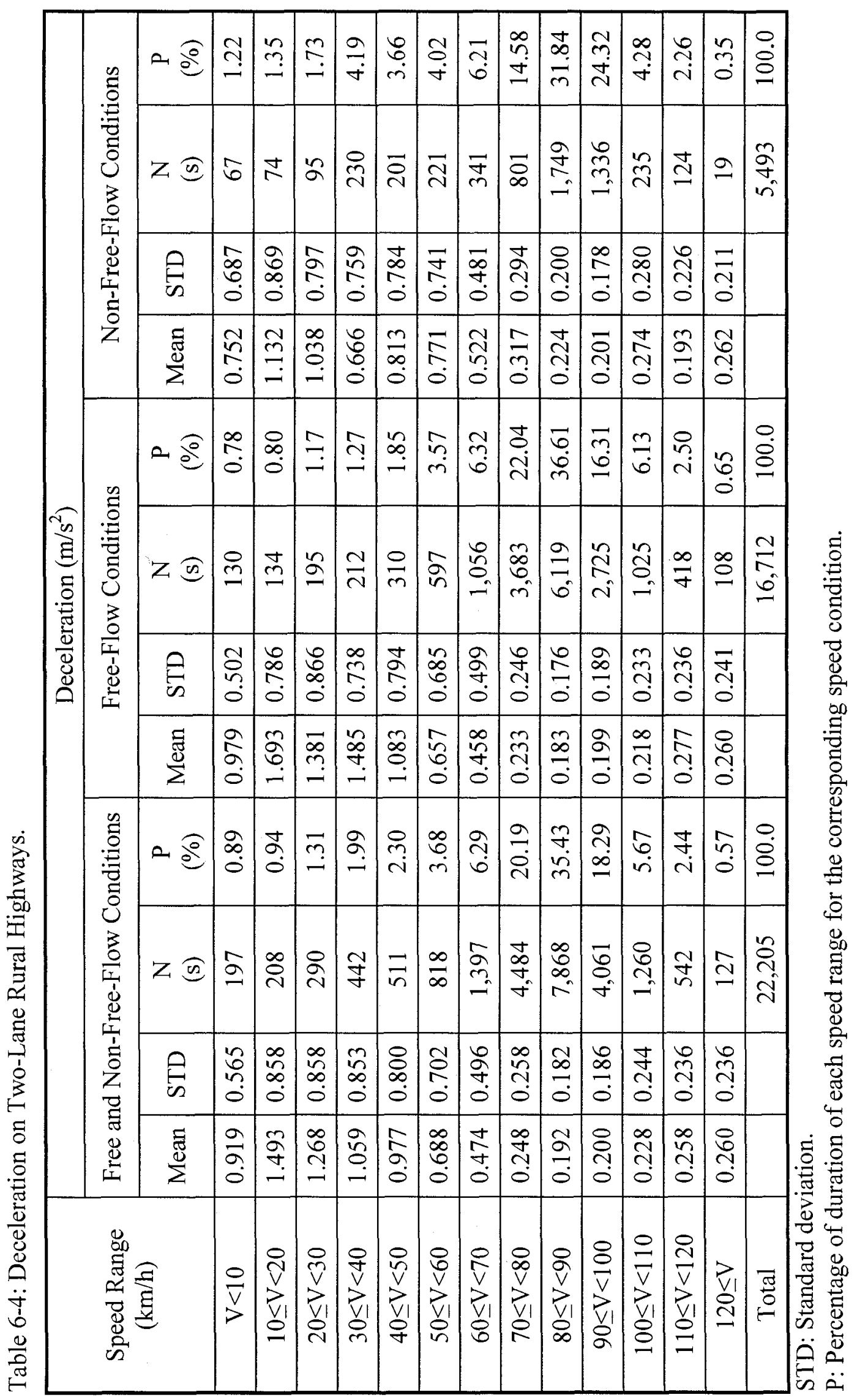




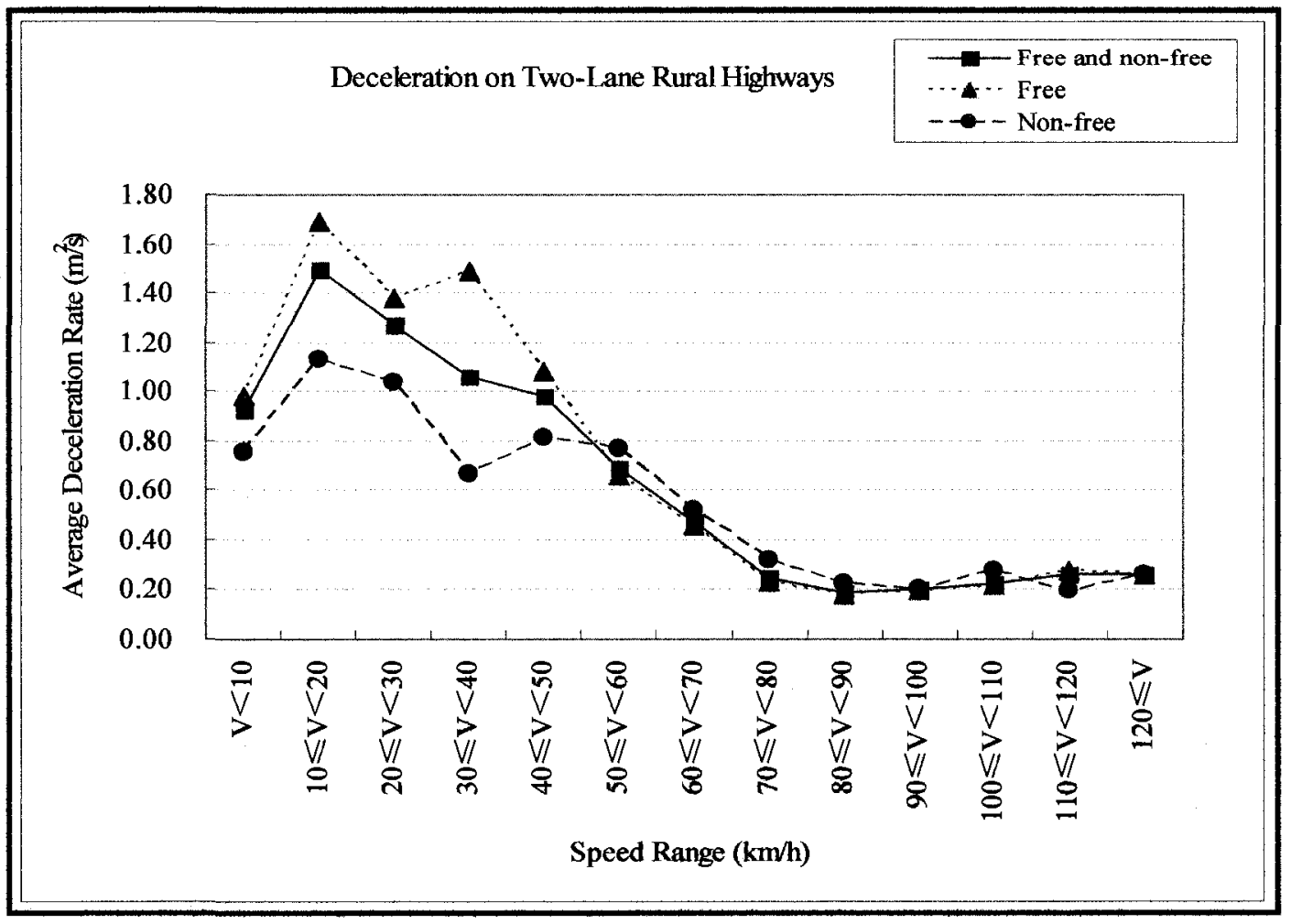

Figure 6-5: Deceleration on Two-Lane Rural Highways.

\subsubsection{Rural Highway (Highway 416)}

Table 6-5 shows the statistics of acceleration rates on Highway 416 and Figure 6-6 illustrates the average acceleration rates associated with driving speeds for the three flow conditions on this road. Table 6-6 and Figure 6-7 show the statistics and the illustration of driver deceleration performance on Highway 416.

As shown in Figure 6-6, the acceleration on this rural freeway had two scenarios; one was relatively stable acceleration behaviour, representing the case when speeds were above $90 \mathrm{~km} / \mathrm{h}$, and the other was acute acceleration behaviour, representing the case when speeds were below $90 \mathrm{~km} / \mathrm{h}$. This indicates that the drivers accelerated quickly as they entered the freeway and tried to reach an appropriate speed on the freeway, and afterwards the drivers kept the vehicle travelling in a stable manner when the vehicle 
speeds were in high ranges in accordance with the prevailing traffic. The figure also shows that there was no evident difference of average acceleration rates between freeflow and non-free-flow conditions in the same speed range, except for the lowest speed range between 60 and $70 \mathrm{~km} / \mathrm{h}$. Such a large difference might have resulted from the small size of acceleration observations in this speed range (refer to Table 6-5). The closeness of accelerations for the two traffic conditions proves, again, that the drivers were still likely to drive at will on the multi-lane freeway with a relatively low traffic volume, where fast leading vehicles did not necessarily affect speed or acceleration of the trailing vehicle although the time headways represented a non-free status.

According to Figure 6-7, it is observed that the drivers usually applied gentle deceleration on Highway 416 in either free-flow or non-free-flow conditions. Moreover, the deceleration used under free-flow conditions was generally softer than that under nonfree-flow conditions. The two observations were in accordance with that of driver deceleration performance on Highway 417. 


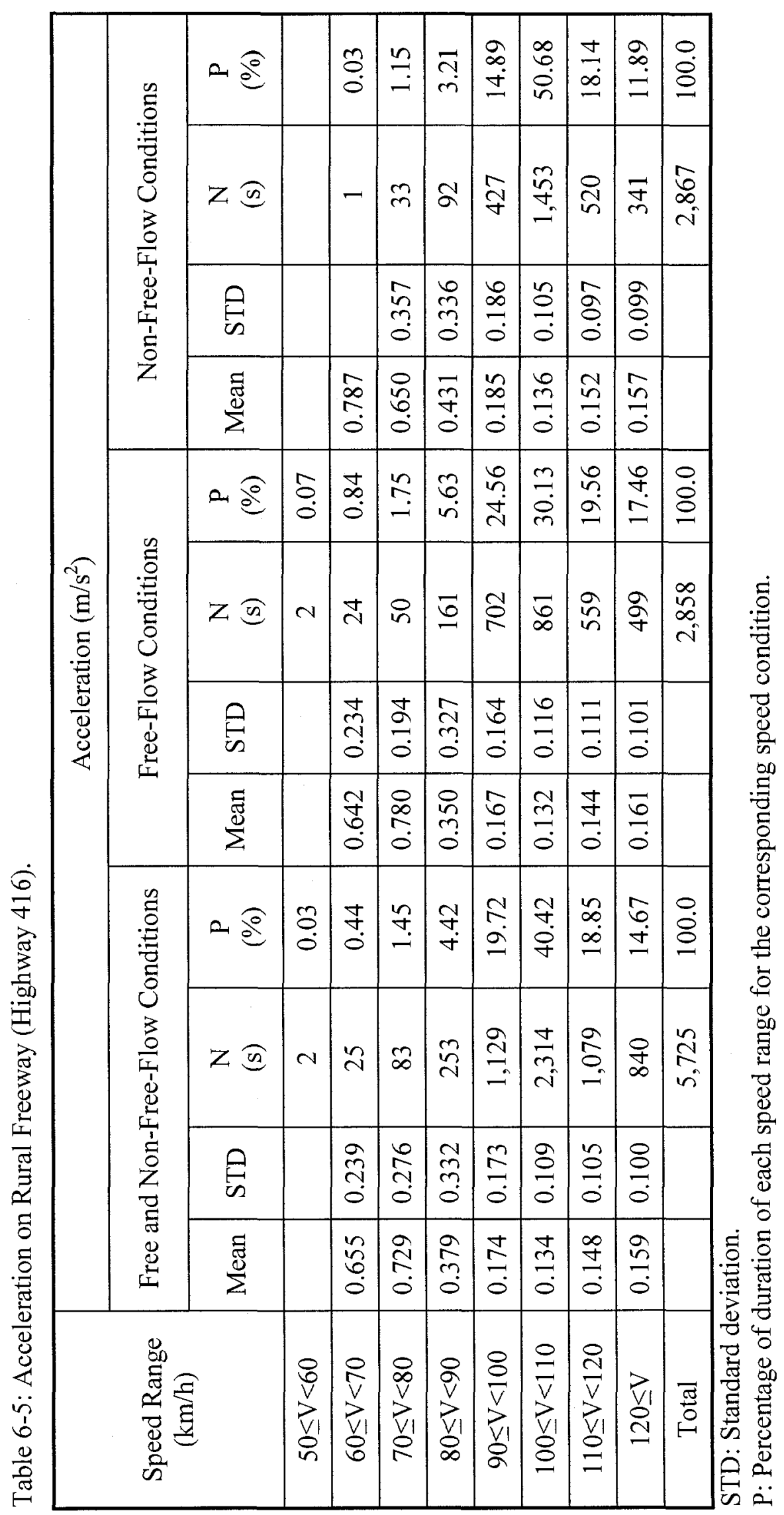




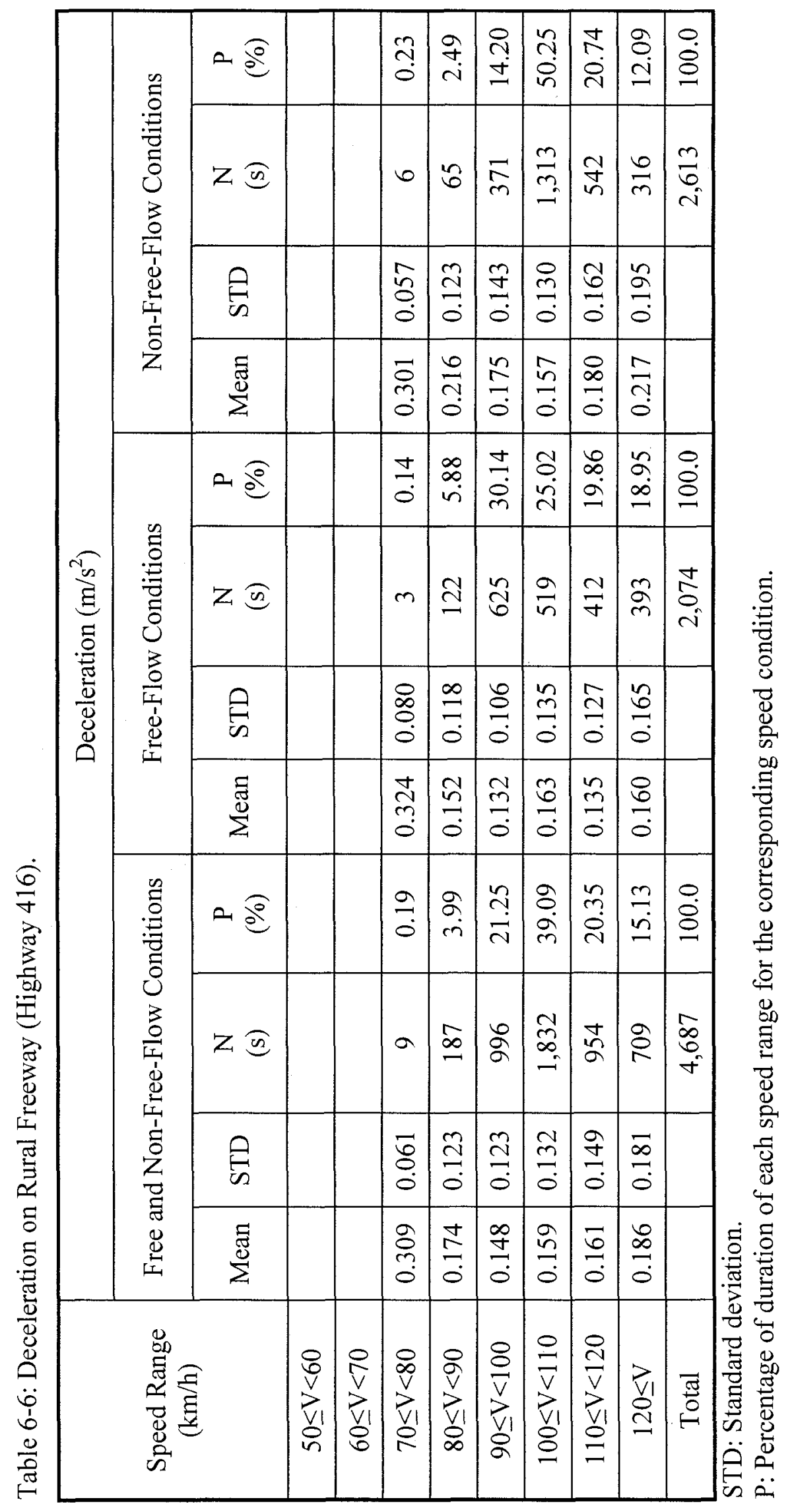




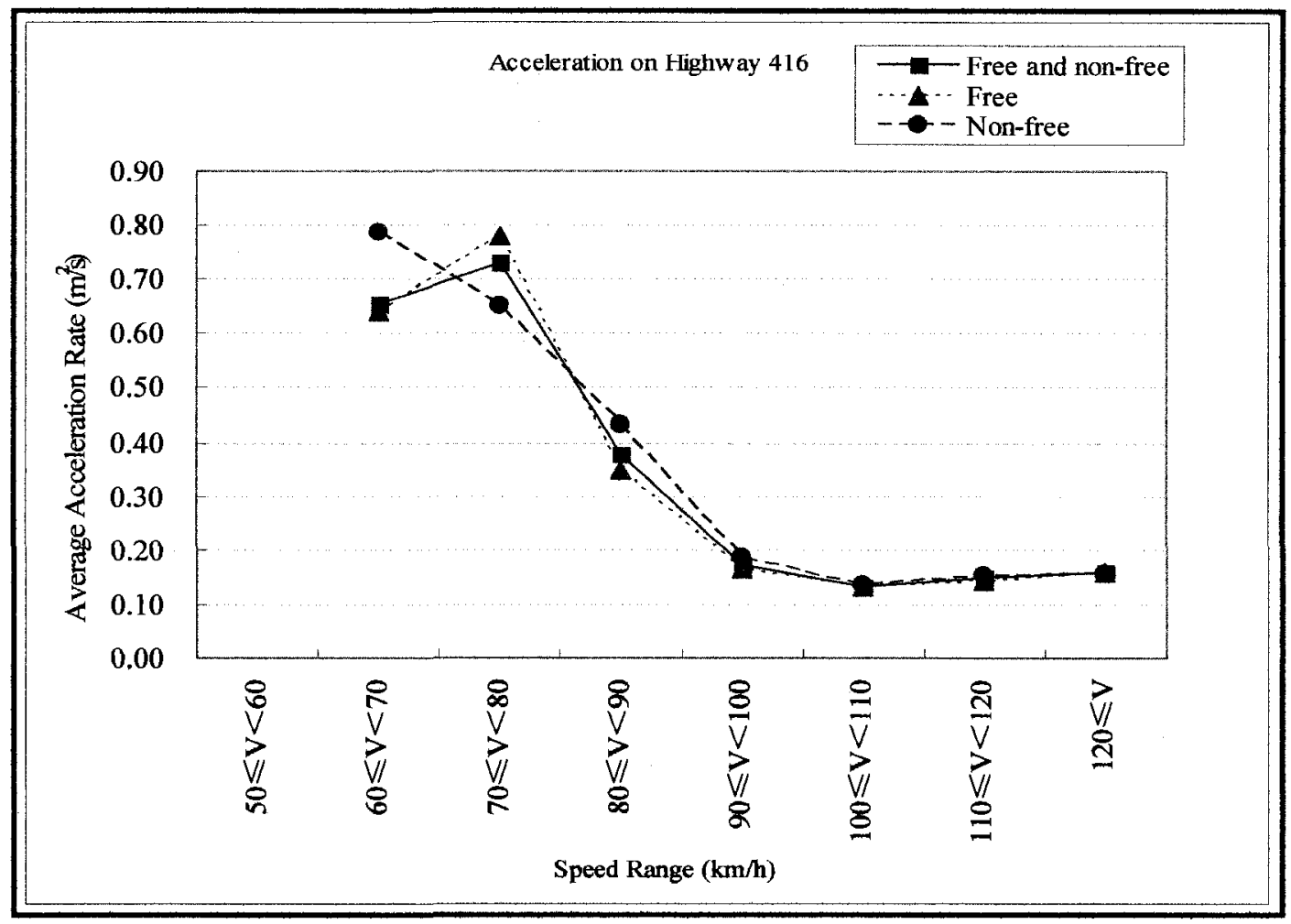

Figure 6-6: Acceleration on Rural Freeway (Highway 416).

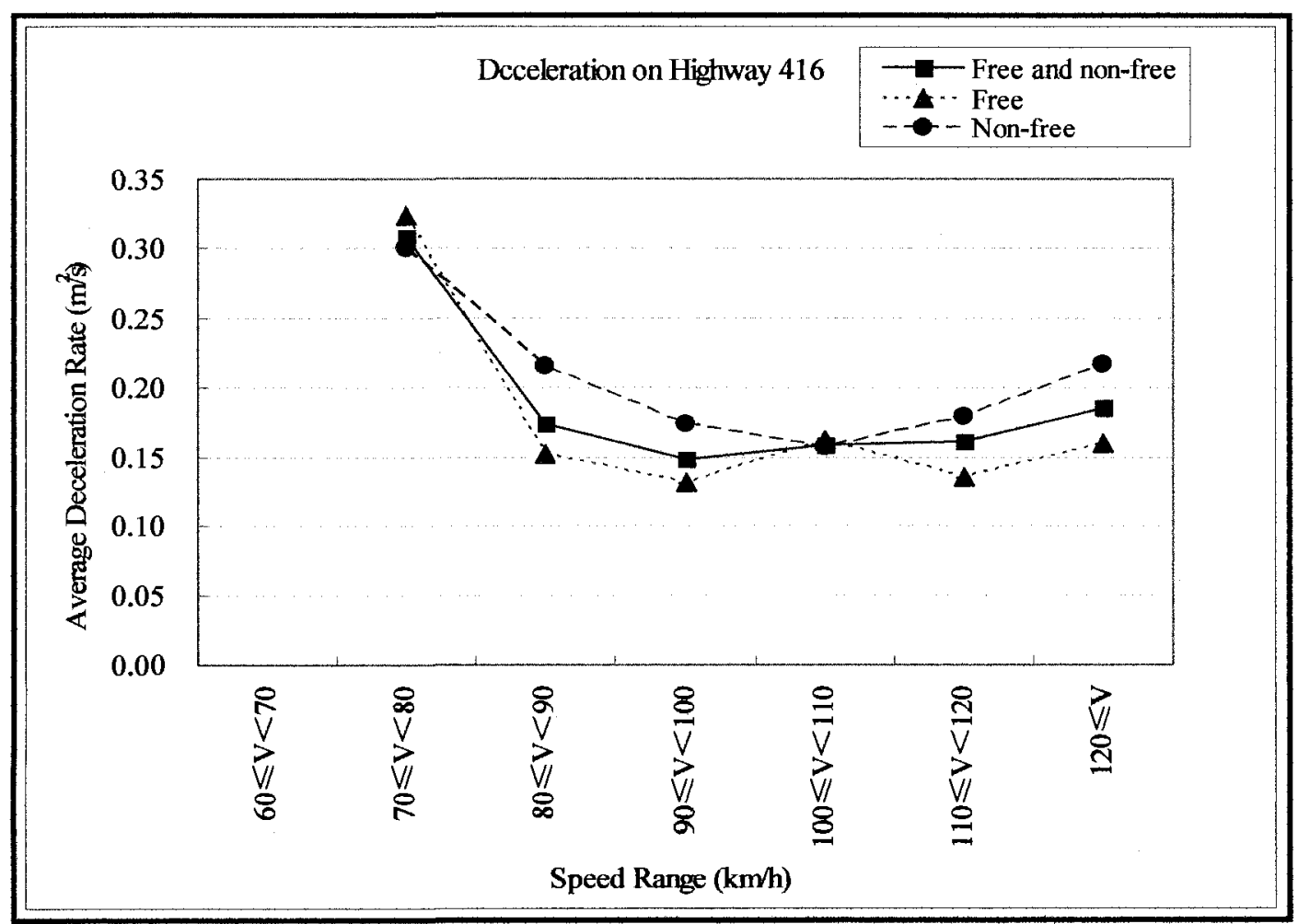

Figure 6-7: Deceleration on Rural Freeway (Highway 416). 


\subsubsection{Urban/Suburban Roads}

Table 6-7 shows the statistics of acceleration rates on the urban/suburban roads under investigation and Figure 6-8 illustrates the average acceleration rates associated with driving speeds for the three flow conditions on these roads. Table 6-8 and Figure 6-9 show the statistics and the illustration of the deceleration on urban/suburban roads.

According to Figure 6-8, the acceleration rates of the driver sample decreased, on average, with the increase of speed on urban/suburban roads when speeds were between 10 and $70 \mathrm{~km} / \mathrm{h}$, and the average acceleration rates were relatively low when speeds were above $70 \mathrm{~km} / \mathrm{h}$. The highest average acceleration rate did not appear in the bottom speed range, but rather in the speed range of 10 to $20 \mathrm{~km} / \mathrm{h}$. Moreover, drivers usually had higher acceleration rates under free-flow conditions than under non-free-flow conditions when speeds were lower than $60 \mathrm{~km} / \mathrm{h}$. However, when speeds were greater than 60 $\mathrm{km} / \mathrm{h}$, the acceleration rates under the two conditions were close to each other and acceleration rates under free-flow conditions were not necessarily greater than those under non-free-flow conditions for the same speed range.

According to Figure 6-9, the highest average deceleration rates appeared in the speed ranges of 10 to $40 \mathrm{~km} / \mathrm{h}$ on urban/suburban roads. Moreover, the figure shows a trend that the average deceleration rates decreased with the increase in speed ranges between 40 and $70 \mathrm{~km} / \mathrm{h}$ and they became relatively stable when speeds were above $70 \mathrm{~km} / \mathrm{h}$. The figure also shows that the drivers usually applied harder deceleration under free-flow conditions than under non-free-flow conditions when speeds were lower than $50 \mathrm{~km} / \mathrm{h}$. The difference was especially substantial in the speed ranges between 10 and $40 \mathrm{~km} / \mathrm{h}$. On the other hand, the average deceleration rates under the two conditions became close to each other when speeds were greater than $50 \mathrm{~km} / \mathrm{h}$. However, the drivers applied relatively higher deceleration rates in the high speed ranges of 90 to $110 \mathrm{~km} / \mathrm{h}$ under nonfree-flow conditions than under free-flow conditions. 


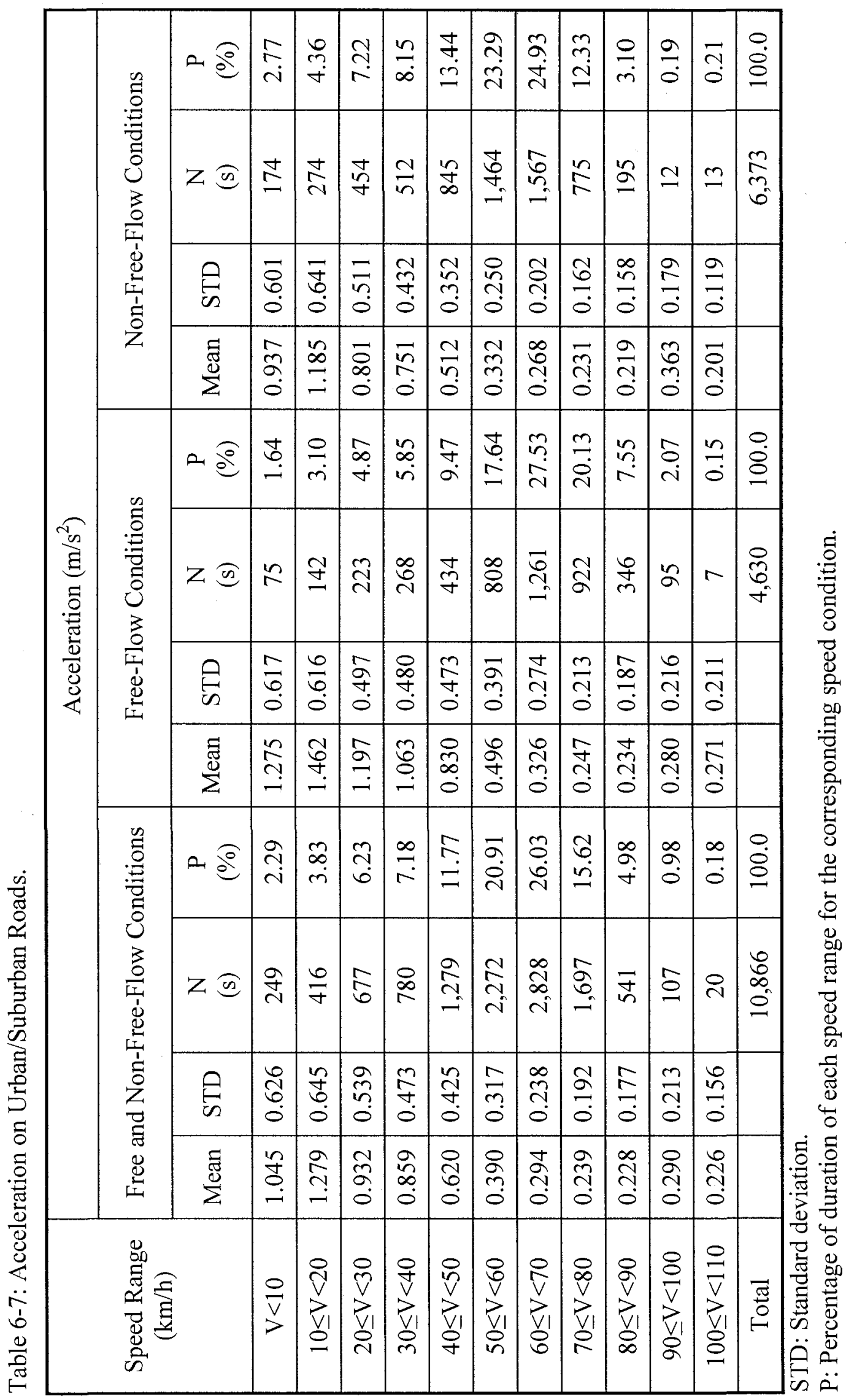




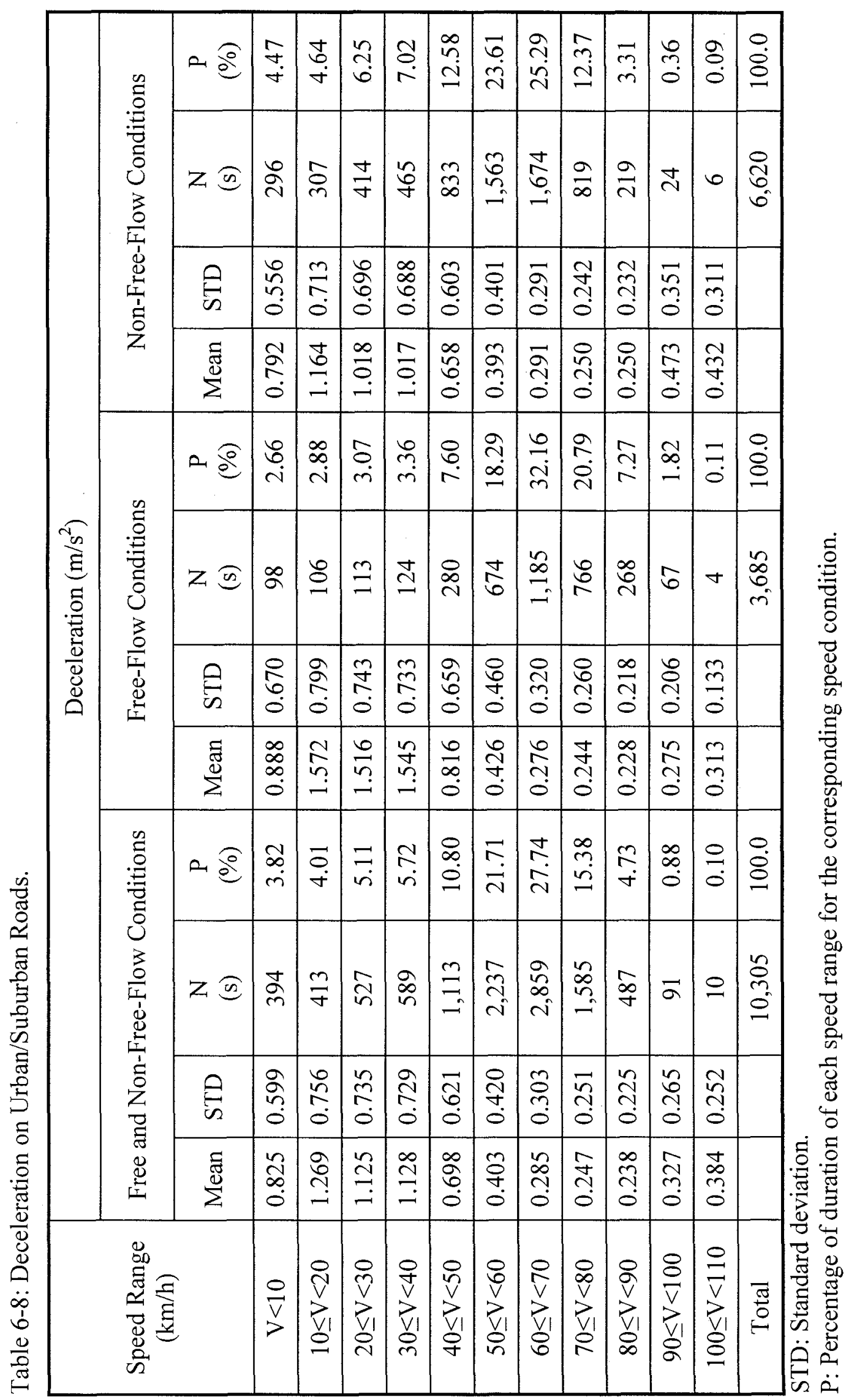




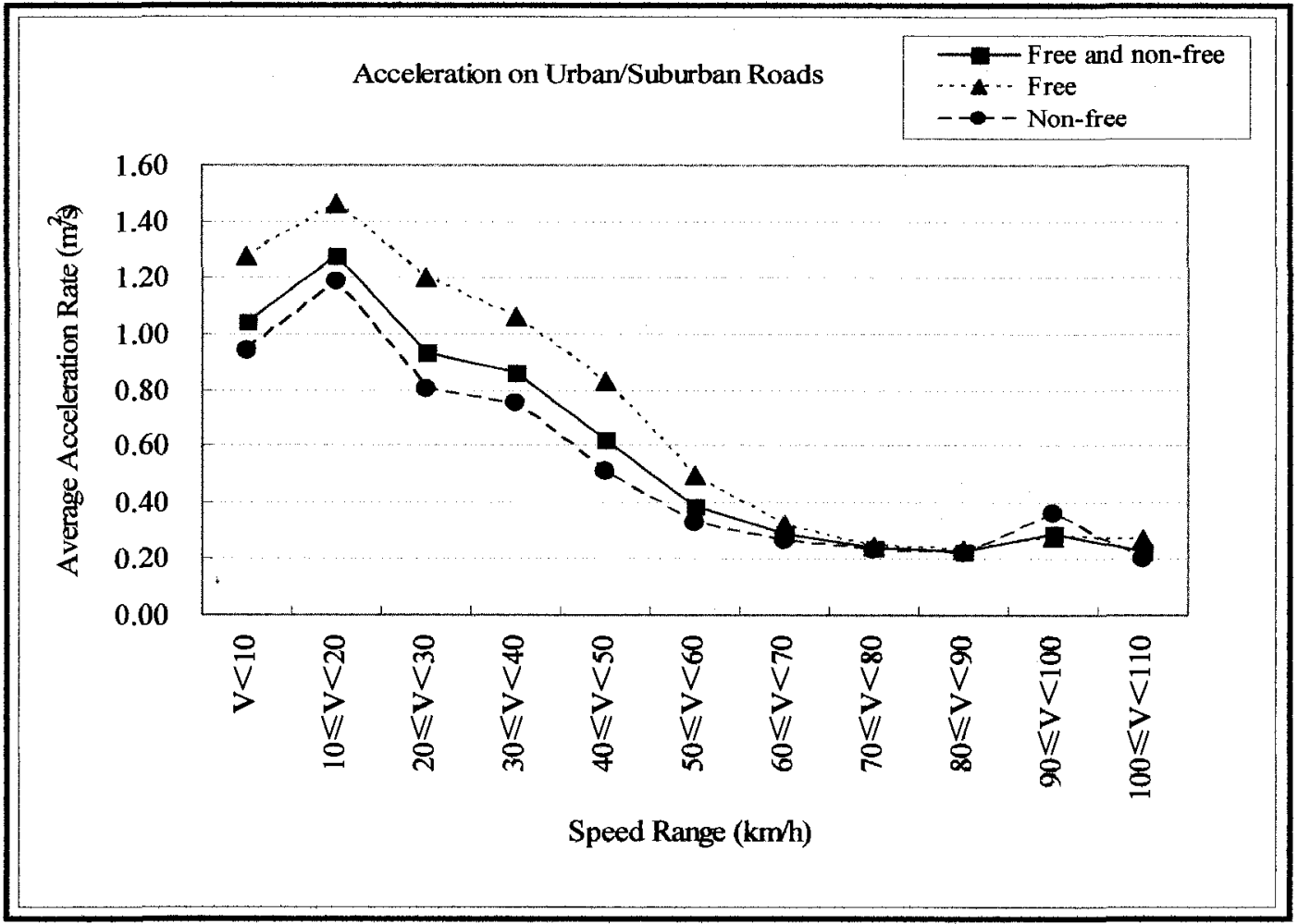

Figure 6-8: Acceleration on Urban/Suburban Roads.

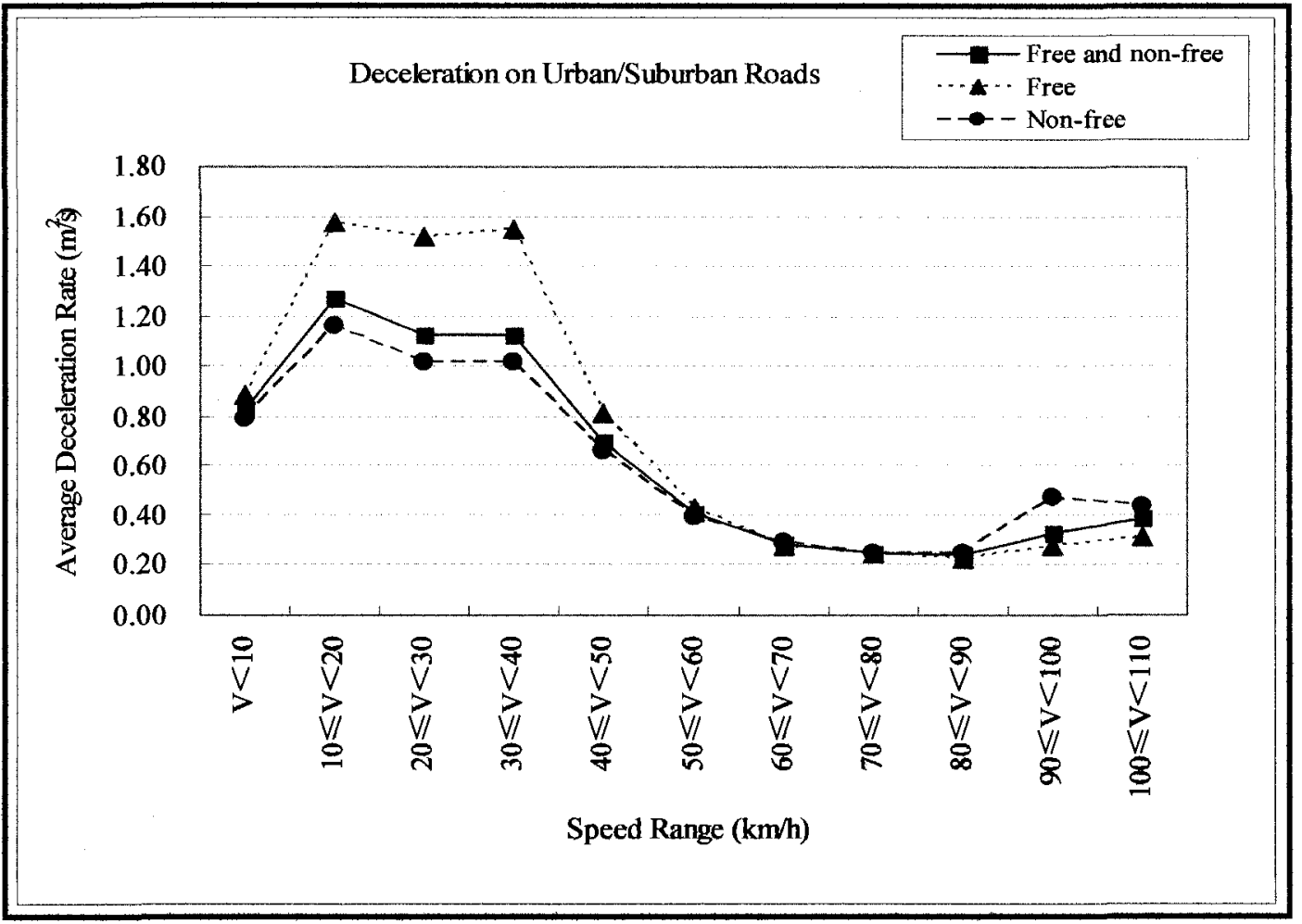

Figure 6-9: Deceleration on Urban/Suburban Roads. 


\subsubsection{Comparison of Acceleration/Deceleration on Different Road Classes}

In order to investigate whether various road environments played a role in affecting driver acceleration and deceleration behaviour, the acceleration and deceleration performances of the driver sample were compared among the four road classes in this study. It is believed that speed conditions in terms of free-flow and non-free-flow would have a significant influence on driver behaviour. Thus the comparisons were conducted for free-flow speeds and non-free-flow speeds separately. Moreover, the cruise states that represent the cases when instantaneous acceleration rates were zero, were also compared to find out in what speeds the drivers were more likely to keep their speeds constant. It should be noted that the statistics presented in this section are mostly the same ones presented in sections 6.2 .1 to 6.2 .4 . The only difference is that this section compiles the findings and re-presents them with road class as the main variable under investigation.

\subsection{5.a Acceleration Behaviour}

Tables 6-9 and 6-10 present the average acceleration rates relevant to the driving speeds on the four road classes for free-flow and non-free-flow conditions, respectively, and these acceleration rates are illustrated in Figure 6-10 and Figure 6-11. The percentages of acceleration duration in the tables were computed using the corresponding time of acceleration operations on each individual road class.

As shown in Figure 6-10, the mean acceleration rates in the same speed ranges were generally close to each other for different road classes under free-flow conditions. In addition, the figure shows a general trend of decreasing acceleration rates with the increase of speed. However, the highest average acceleration was not associated with the lowest speed range, which corresponds to acceleration after a complete stop. Rather the 
highest acceleration rate generally took place corresponding to the speed range of 10 to $20 \mathrm{~km} / \mathrm{h}$ after the vehicle had gained some speed. Moreover, the acceleration rates on all road classes became stable when drivers reached high speed ranges (higher than $80 \mathrm{~km} / \mathrm{h}$ ). One exception is the average acceleration rates on freeways in the speed range of 60 to 80 $\mathrm{km} / \mathrm{h}$, which were much higher than those for urban/suburban roads and two-lane rural highways. Such high acceleration rates were adopted as the drivers entered the freeway and tried to reach an appropriate speed on the freeway. In contrast, the same speed range on two-lane rural highways and urban/suburban roads represented high speed ranges where drivers had no reason to adopt hard acceleration.

As shown in Figure 6-11, mean acceleration rates under non-free-flow conditions were generally lower than those under free-flow conditions. Therefore, the data suggest that the impediment of a leading vehicle may constrain the driver's choice of acceleration. Yet, the same general trend of decreasing acceleration rate with the increase of speed can also be observed under non-free-flow conditions. Also, the highest acceleration rate corresponded to the speed range of 10 to $20 \mathrm{~km} / \mathrm{h}$. On the other hand, there was more variation in the drivers' mean acceleration rate with the road class under non-free-flow conditions. 


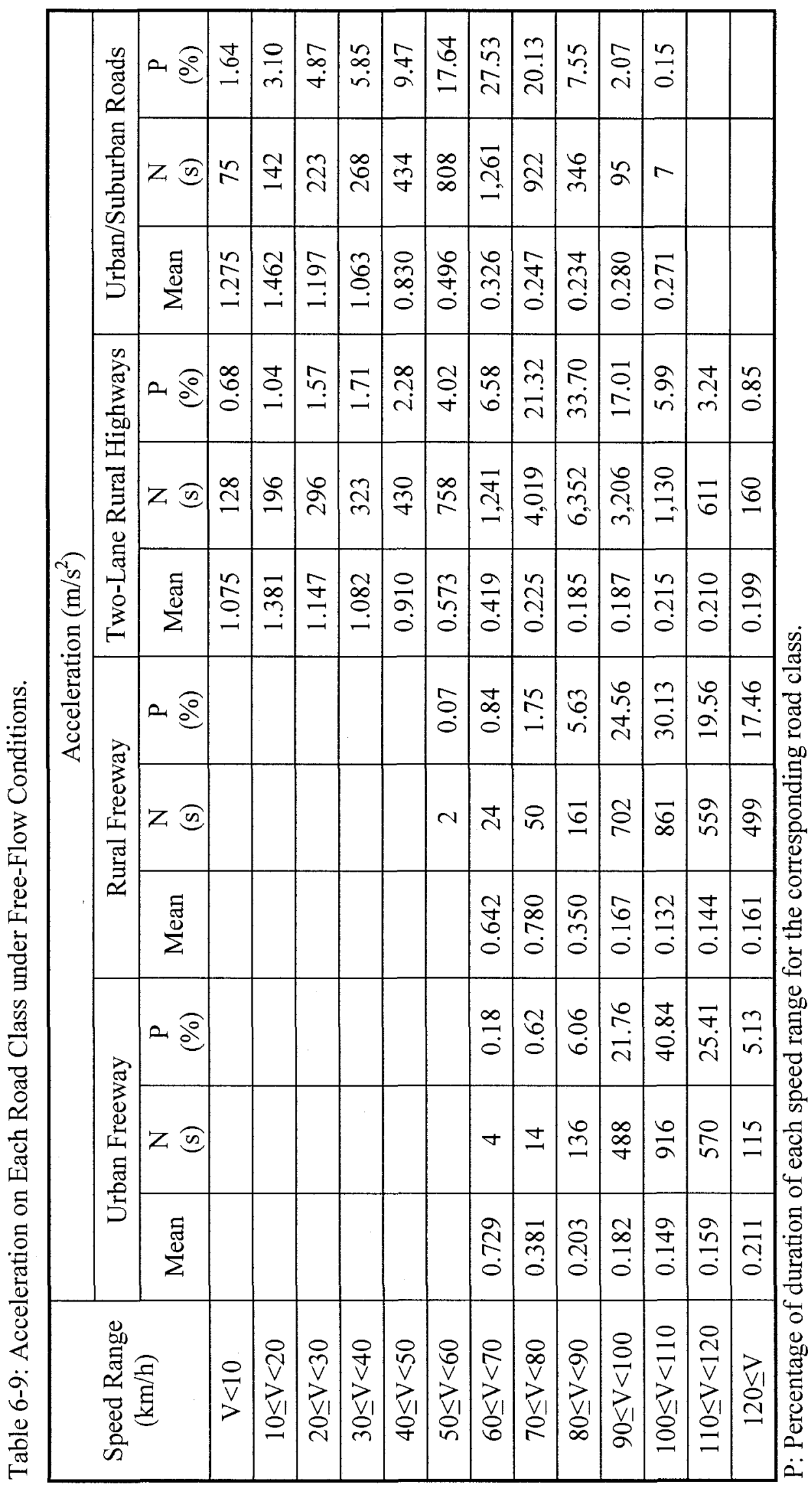




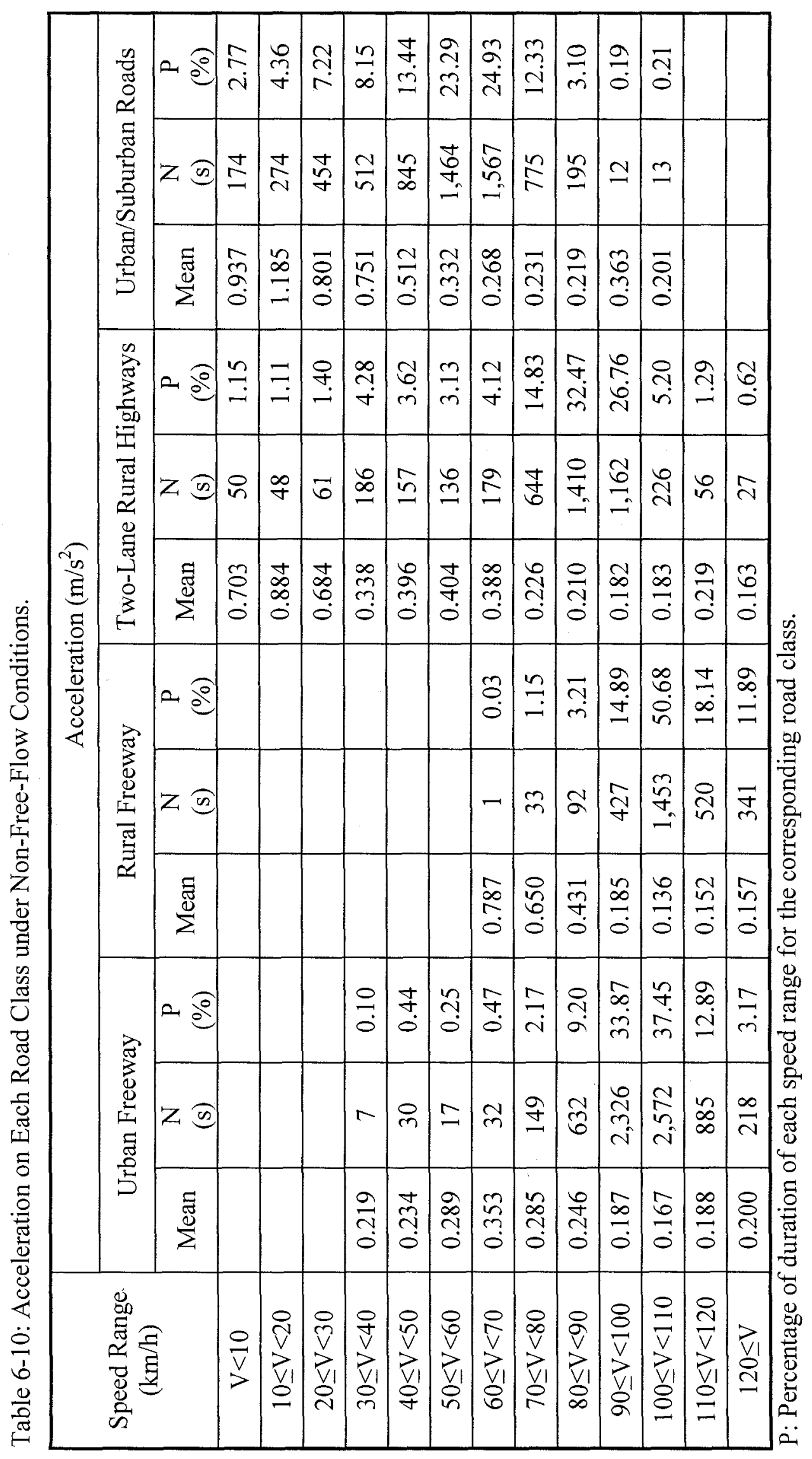




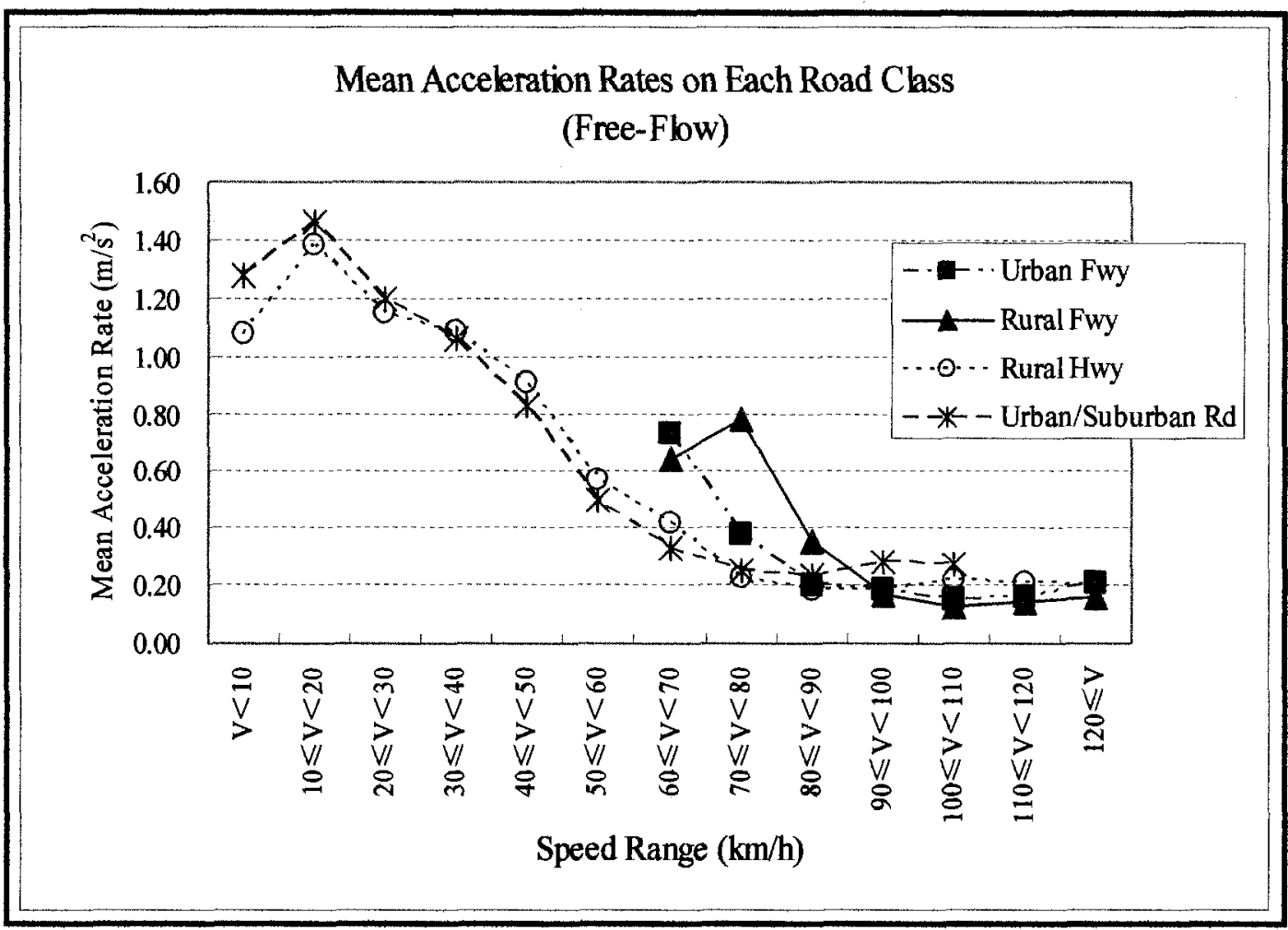

Figure 6-10: Acceleration on Each Road Class under Free-Flow Conditions.

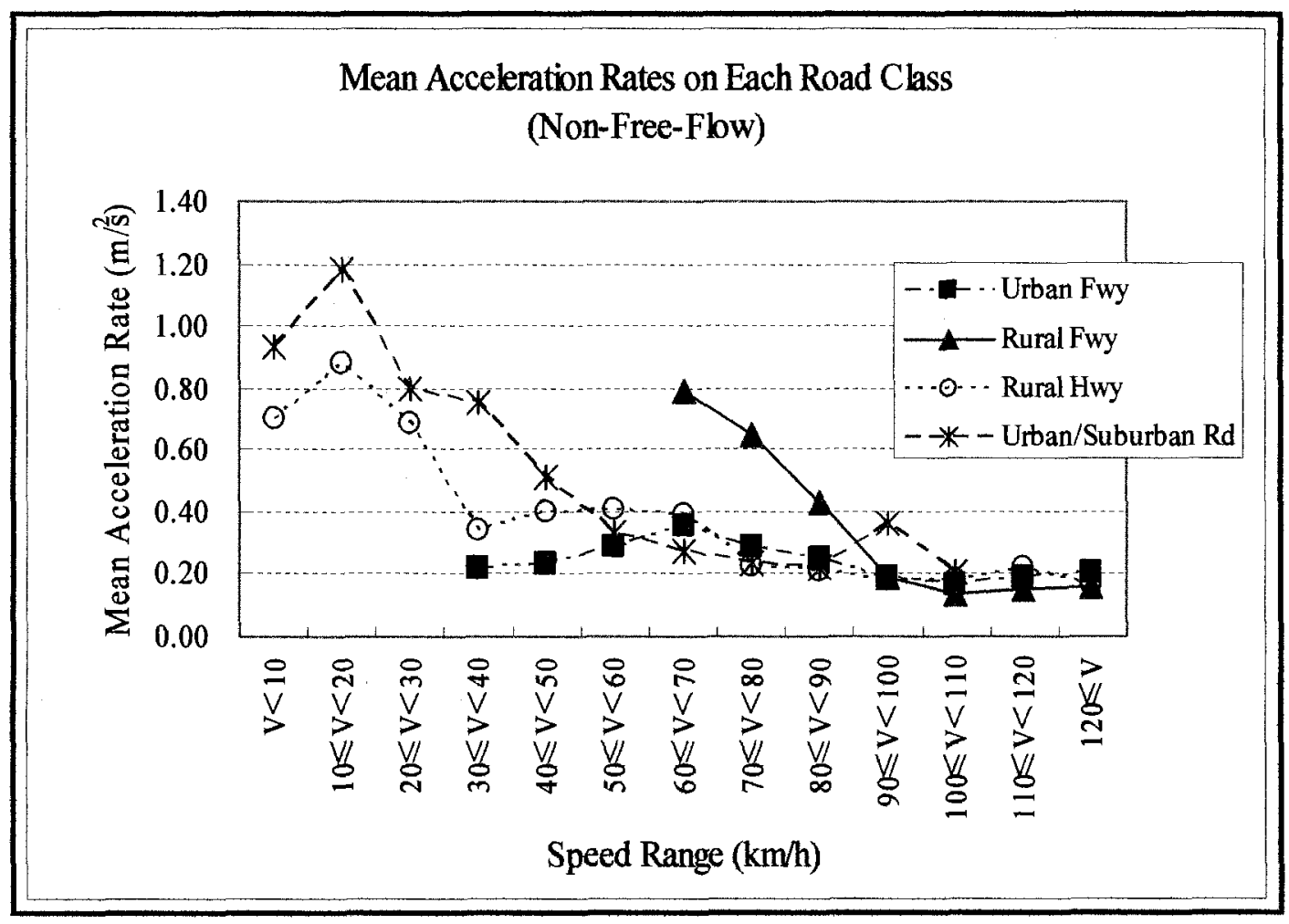

Figure 6-11: Acceleration on Each Road Class under Non-Free-Flow Conditions. 


\subsection{5.b Deceleration Behaviour}

Tables 6-11 and 6-12 present the average deceleration rates relevant to the driving speeds on the four road classes for free-flow and non-free-flow conditions, respectively. These deceleration rates are also illustrated in Figure 6-12 and Figure 6-13. The percentages of deceleration duration in the tables were computed using the corresponding time of deceleration operations on each individual road class.

Referring to Figures 6-12 and 6-13, the deceleration behaviour on the four road classes exhibited similar trends to acceleration. Namely, under free-flow conditions, the average deceleration generally decreased with the increase of speed when speeds were lower than $80 \mathrm{~km} / \mathrm{h}$, and they tended to be stable when speed exceeded $80 \mathrm{~km} / \mathrm{h}$. Nevertheless, the highest average deceleration rate did not exist in the lowest speed range that represents the moment before a complete stop. Rather, the hardest deceleration usually took place in the speed ranges of 10 to $20 \mathrm{~km} / \mathrm{h}$. Furthermore, the average deceleration rates for the same speed range were close for all road classes under free-flow conditions, whereas greater variations occurred between the average deceleration rates for different road classes under non-free-flow conditions. Additionally, the average deceleration rates corresponding to each speed range were substantially lower than the comfortable deceleration rates of $3.0 \mathrm{~m} / \mathrm{s}^{2}$ suggested by ITE (1992). 


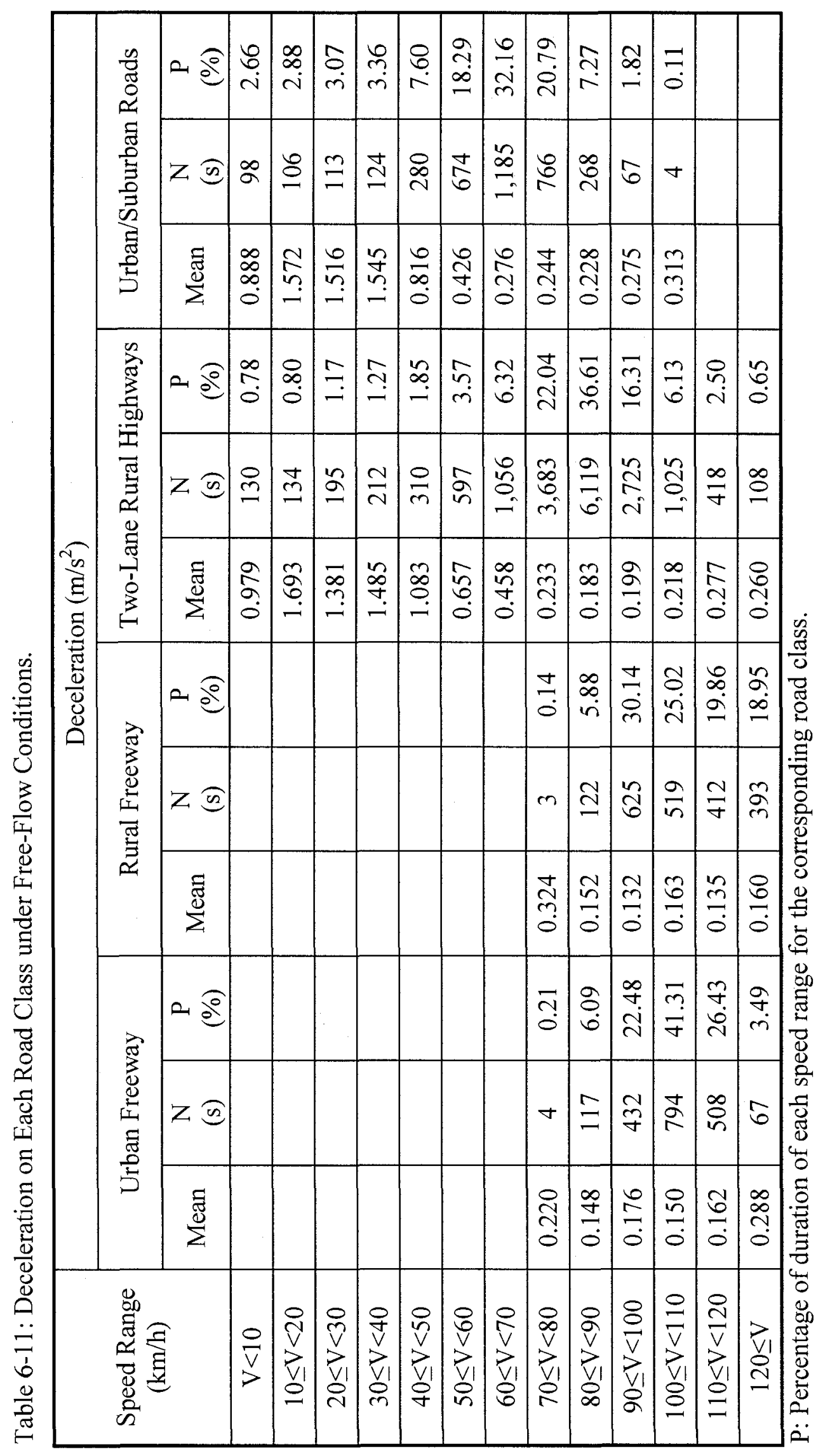

in 


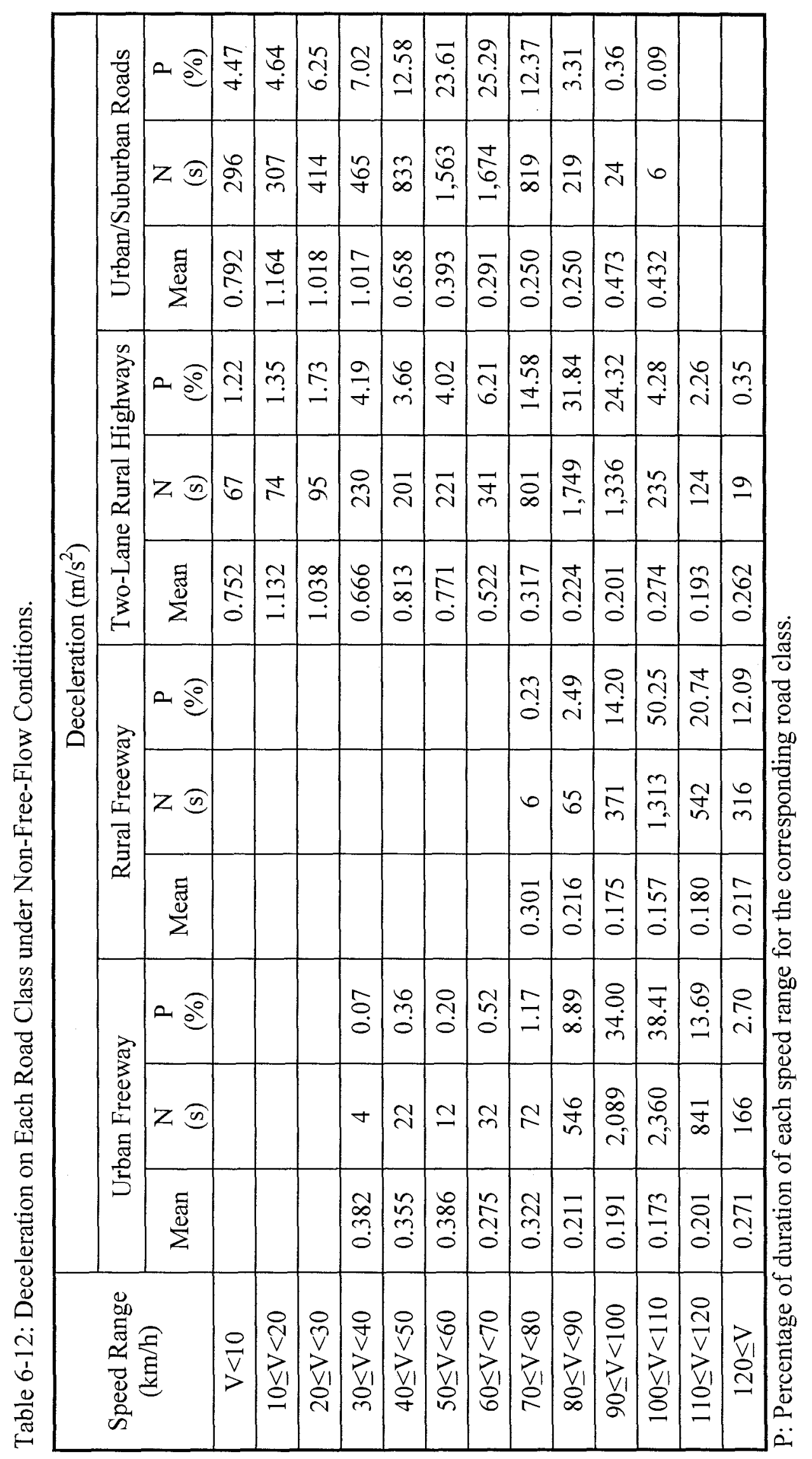




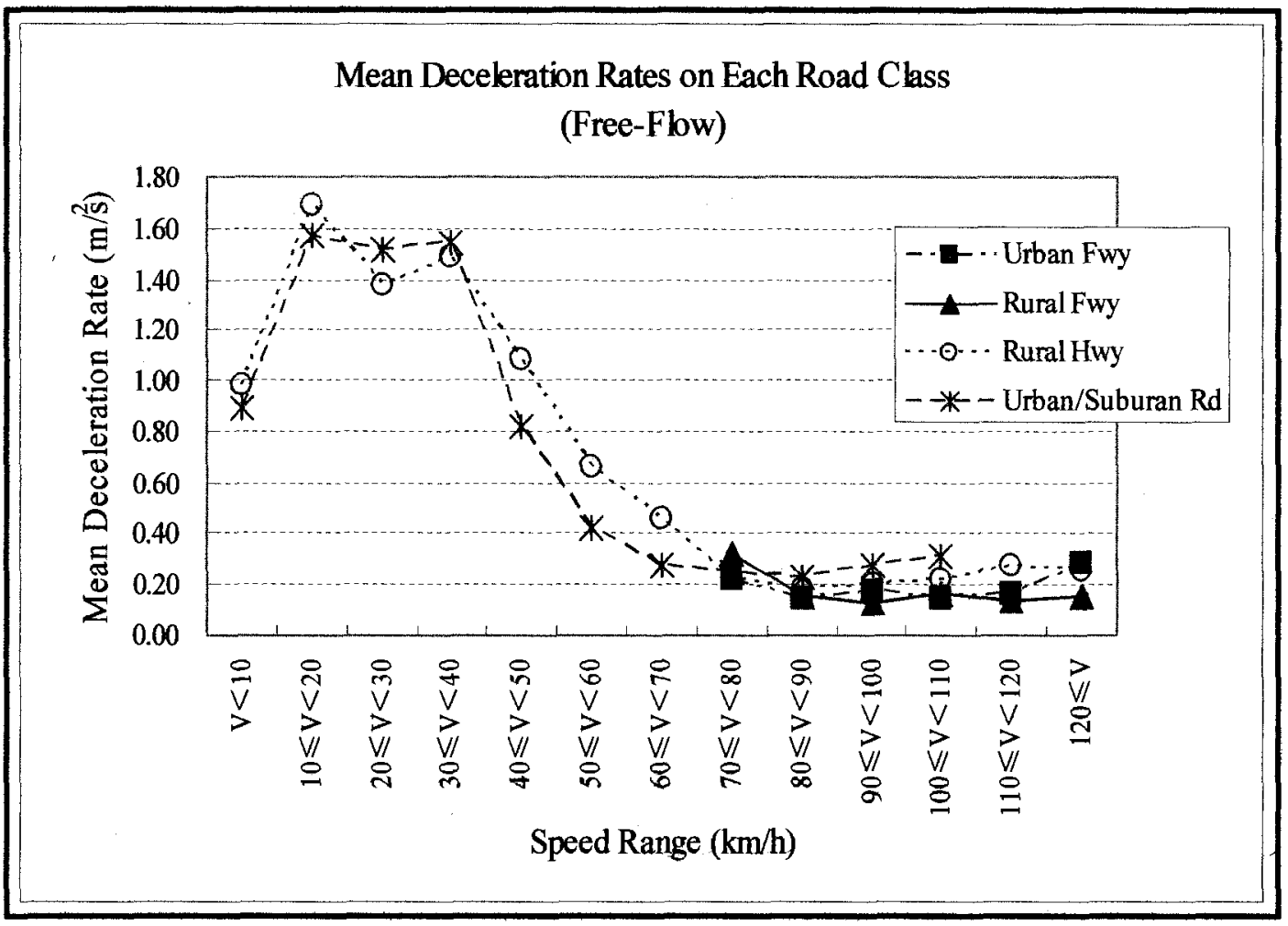

Figure 6-12: Deceleration on Each Road Class under Free-Flow Conditions.

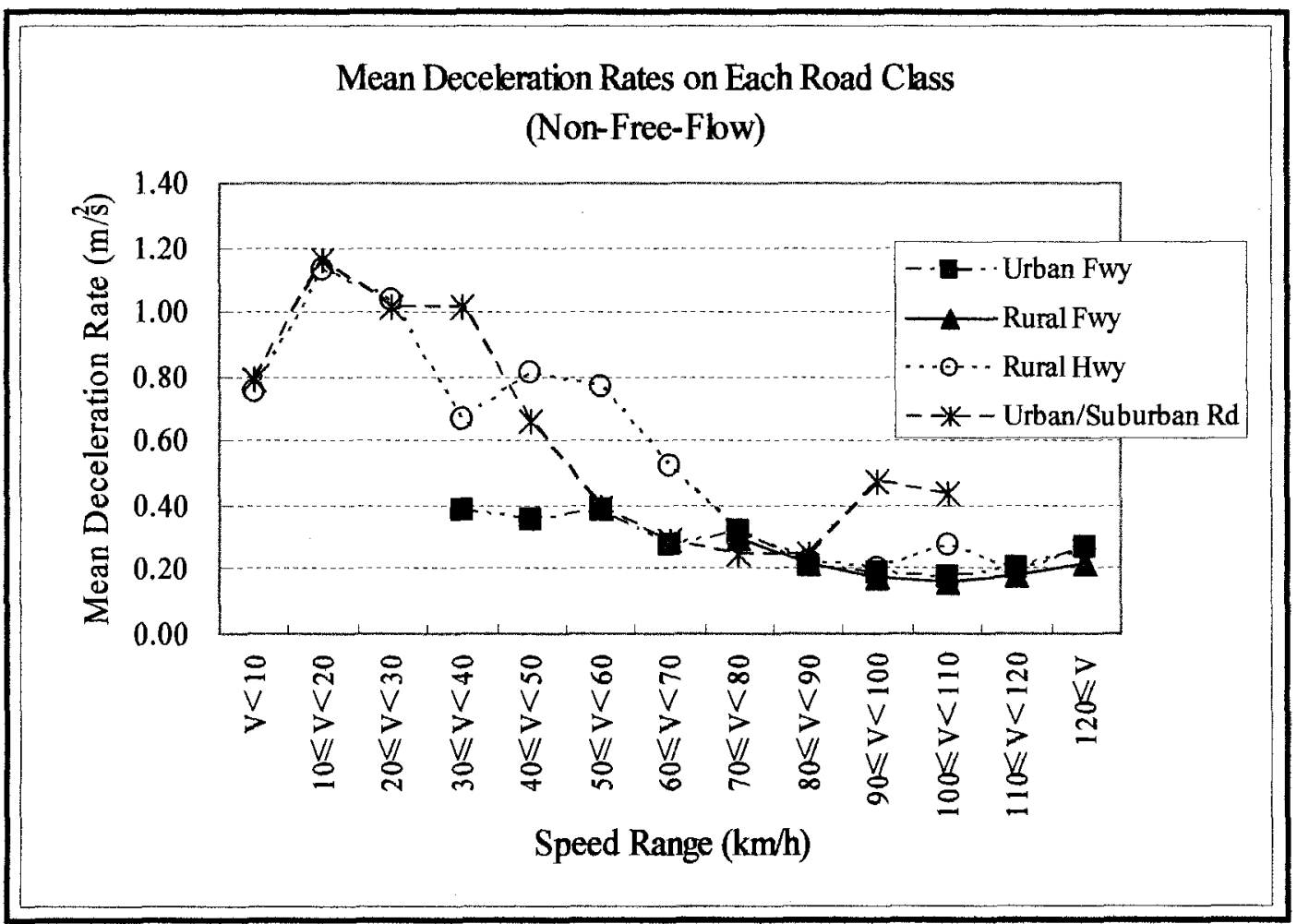

Figure 6-13: Deceleration on Each Road Class under Non-Free-Flow Conditions. 


\subsection{5.c Cruise mode}

Table 6-13 shows the cruise durations on each road class under mixed-flow conditions and the corresponding percentages of cruise durations are illustrated in Figure 6-14. It should be noted that the cruise durations in the bottom speed range on two-lane rural highways and urban/suburban roads do not include the vehicle standstill time at intersections. According to Figure 6-14, the cruise status happened most frequently in the high speed ranges on corresponding road classes. For example, on Highway 417 cruise was most likely to occur in the speed ranges of 90 to $120 \mathrm{~km} / \mathrm{h}$, and on Highway 416 cruise occurred when speeds exceeded $90 \mathrm{~km} / \mathrm{h}$. These observations were generally in accordance with the speed distributions on the two freeways. For two-lane rural highways and urban/suburban roads where many low speeds took place, little cruise duration, however, appeared in these low speed ranges (less than $40 \mathrm{~km} / \mathrm{h}$ ). This observation suggests that the drivers often changed their speeds when the vehicle approached intersections or left intersections before achieving appropriate speeds. 
Table 6-13: Cruise Distribution on Each Road Class under Mixed-Flow Conditions.

\begin{tabular}{|c|c|c|c|c|c|c|c|c|}
\hline \multirow{3}{*}{$\begin{array}{c}\text { Speed } \\
\text { Range } \\
(\mathrm{km} / \mathrm{h})\end{array}$} & \multicolumn{2}{|c|}{$\begin{array}{c}\text { Urban } \\
\text { Freeway }\end{array}$} & \multicolumn{2}{c|}{$\begin{array}{c}\text { Rural } \\
\text { Freeway }\end{array}$} & \multicolumn{2}{c|}{$\begin{array}{c}\text { Two-Lane } \\
\text { Rural Highways }\end{array}$} & \multicolumn{2}{|c|}{$\begin{array}{c}\text { Urban/Suburban } \\
\text { Roads }\end{array}$} \\
\cline { 2 - 9 } & $\mathrm{N}(\mathrm{s})$ & $\mathrm{P}(\%)$ & $\mathrm{N}(\mathrm{s})$ & $\mathrm{P}(\%)$ & $\mathrm{N}(\mathrm{s})$ & $\mathrm{P}(\%)$ & $\mathrm{N}(\mathrm{s})$ & $\mathrm{P}(\%)$ \\
\hline $\mathrm{V}<10^{*}$ & & & & & 14 & 0.13 & 26 & 0.84 \\
\hline $10 \leq \mathrm{V}<20$ & & & & & 3 & 0.03 & 16 & 0.51 \\
\hline $20 \leq \mathrm{V}<30$ & & & & & 6 & 0.05 & 34 & 1.09 \\
\hline $30 \leq \mathrm{V}<40$ & 2 & 0.04 & & & 88 & 0.80 & 46 & 1.48 \\
\hline $40 \leq \mathrm{V}<50$ & 7 & 0.14 & & & 53 & 0.48 & 198 & 6.37 \\
\hline $50 \leq \mathrm{V}<60$ & 6 & 0.12 & & & 159 & 1.44 & 693 & 22.30 \\
\hline $60 \leq \mathrm{V}<70$ & 9 & 0.18 & 1 & 0.03 & 345 & 3.12 & 1,022 & 32.88 \\
\hline $70 \leq \mathrm{V}<80$ & 48 & 0.98 & 3 & 0.09 & 2,368 & 21.42 & 777 & 25.00 \\
\hline $80 \leq \mathrm{V}<90$ & 383 & 7.83 & 92 & 2.72 & 4,626 & 41.84 & 251 & 8.08 \\
\hline $90 \leq \mathrm{V}<100$ & 1,600 & 32.71 & 713 & 21.06 & 2,408 & 21.78 & 38 & 1.22 \\
\hline $100 \leq \mathrm{V}<110$ & 1,965 & 40.18 & 1,282 & 37.87 & 610 & 5.52 & 7 & 0.23 \\
\hline $110 \leq \mathrm{V}<120$ & 754 & 15.42 & 719 & 21.24 & 280 & 2.53 & & \\
\hline $120 \leq \mathrm{V}$ & 117 & 2.39 & 575 & 16.99 & 96 & 0.87 & & \\
\hline Total & 4,891 & 100.0 & 3,385 & 100.0 & 11,056 & 100.0 & 3,108 & 100.0 \\
\hline
\end{tabular}

$*$ : The standstill time is not included in the cruise duration.

P: Percentage of duration of each speed range for the corresponding road class. 


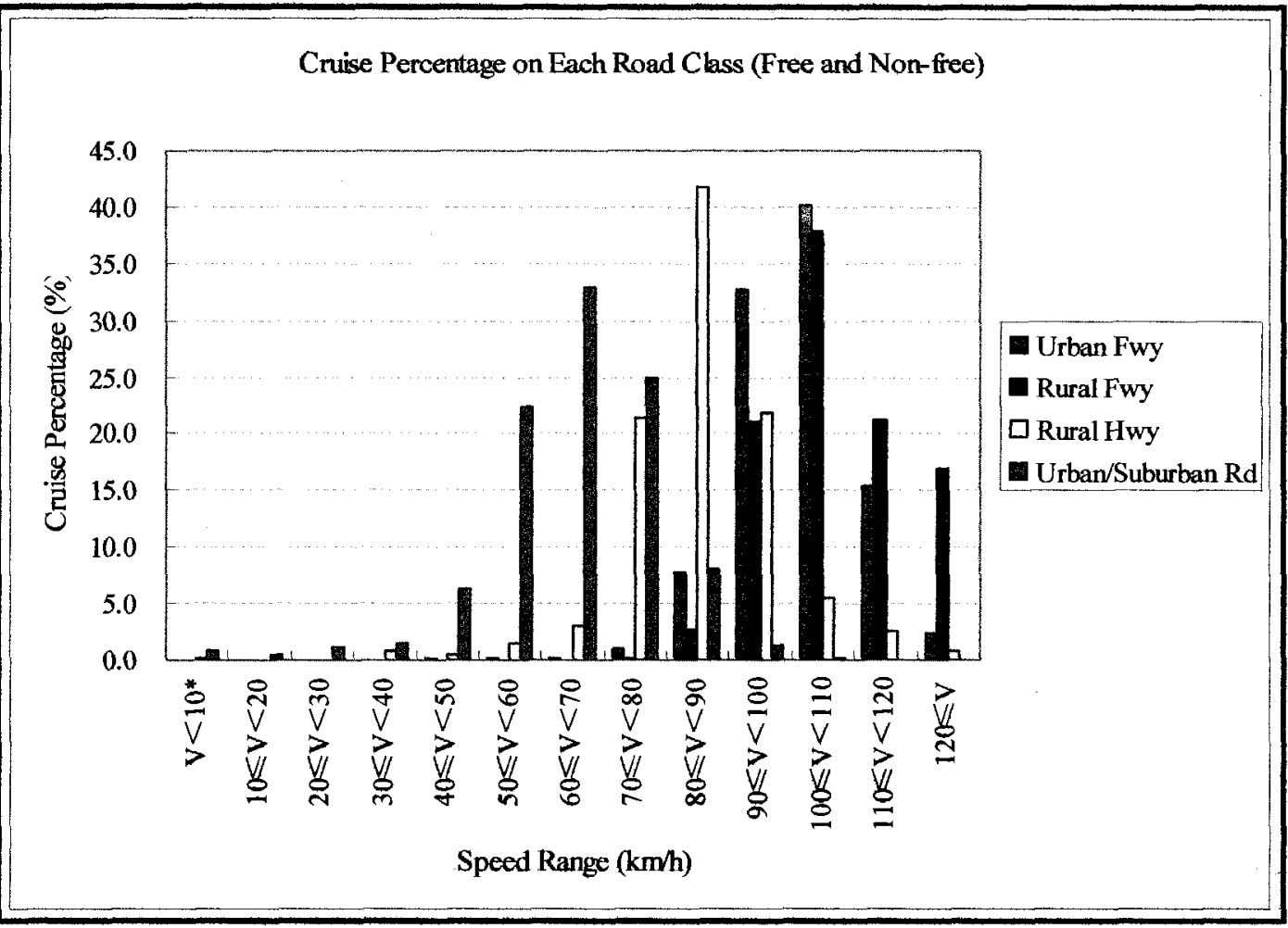

Figure 6-14: Cruise Distribution on Each Road Class under Mixed-Flow Conditions.

\subsection{Acceleration/Deceleration Behaviour of Different Driver Types}

In order to learn if the different driver types, as categorized based on their aggressiveness, had distinct characteristics in using their throttle and brake pedal in general driving conditions, the acceleration/deceleration behaviour of the three types of drivers was compared for mixed flow, free-flow and non-free-flow conditions. The average acceleration/deceleration rates were computed for each driver type on all roads combined and for different speed ranges at $20 \mathrm{~km} / \mathrm{h}$ intervals.

The differences of the acceleration and deceleration performances between the three types of drivers showed a similar general trend for these three flow conditions. Therefore, a detailed explanation here is only directed at the comparison of acceleration/deceleration behaviour between different driver types for the mixed flow condition, which reflects the 
natural environment in a driver's daily driving. The tables and figures related to those comparisons for free-flow and non-free-flow conditions are provided in Appendix B.

\subsubsection{Comparison of Acceleration Behaviour}

Table 6-14 presents the statistics of acceleration corresponding to each speed range under mixed flow conditions for the three driver types. The mean acceleration rates, illustrated in Figure 6-15, provide a better display of the acceleration performance of these driver types. As shown in Figure 6-15, the difference of acceleration behaviour between the three driver types was evident. Generally, aggressive drivers were more likely to apply harder acceleration than the common and defensive drivers, whereas defensive drivers preferred to accelerate their vehicle in a gentler manner compared with the other two driver types. This difference was more significant in the middle speed ranges between 20 and $80 \mathrm{~km} / \mathrm{h}$. On the other hand, the difference was less evident in either high speed ranges (speed greater than $80 \mathrm{~km} / \mathrm{h}$ ), where drivers had reached their maximum speed for most of the trip, or the lowest speed range (speed less than $20 \mathrm{~km} / \mathrm{h}$ ), where drivers were starting after a complete stop and were likely impeded by a leading vehicle. 
Table 6-14: Statistics of Acceleration of the Three Driver Types under Mixed Flow Conditions.

\begin{tabular}{|c|c|c|c|c|c|c|c|}
\hline \multirow{2}{*}{$\begin{array}{c}\text { Driver } \\
\text { Type }\end{array}$} & $\begin{array}{c}\text { Acceleration } \\
\text { Parameter } \\
\left(\mathrm{m} / \mathrm{s}^{2}\right)\end{array}$ & \multicolumn{6}{|c|}{ Speed Range $(\mathrm{km} / \mathrm{h})$} \\
\cline { 3 - 8 } & $\mathrm{V}<20$ & $20 \leq \mathrm{V}<40$ & $40 \leq \mathrm{V}<60$ & $60 \leq \mathrm{V}<80$ & $80 \leq \mathrm{V}<100$ & $\mathrm{~V} \geq 100$ \\
\hline \multirow{2}{*}{ Aggressive } & Mean & 1.158 & 1.149 & 0.656 & 0.411 & 0.286 & 0.206 \\
\cline { 3 - 8 } & $\mathrm{STD}$ & 0.650 & 0.552 & 0.468 & 0.312 & 0.224 & 0.148 \\
\hline \multirow{2}{*}{ Common } & Mean & 1.180 & 0.911 & 0.522 & 0.279 & 0.185 & 0.149 \\
\cline { 2 - 8 } & $\mathrm{STD}$ & 0.618 & 0.483 & 0.390 & 0.231 & 0.143 & 0.109 \\
\hline \multirow{2}{*}{ Defensive } & Mean & 1.054 & 0.717 & 0.422 & 0.223 & 0.172 & 0.189 \\
\cline { 2 - 8 } & $\mathrm{STD}$ & 0.642 & 0.562 & 0.346 & 0.178 & 0.134 & 0.123 \\
\hline
\end{tabular}

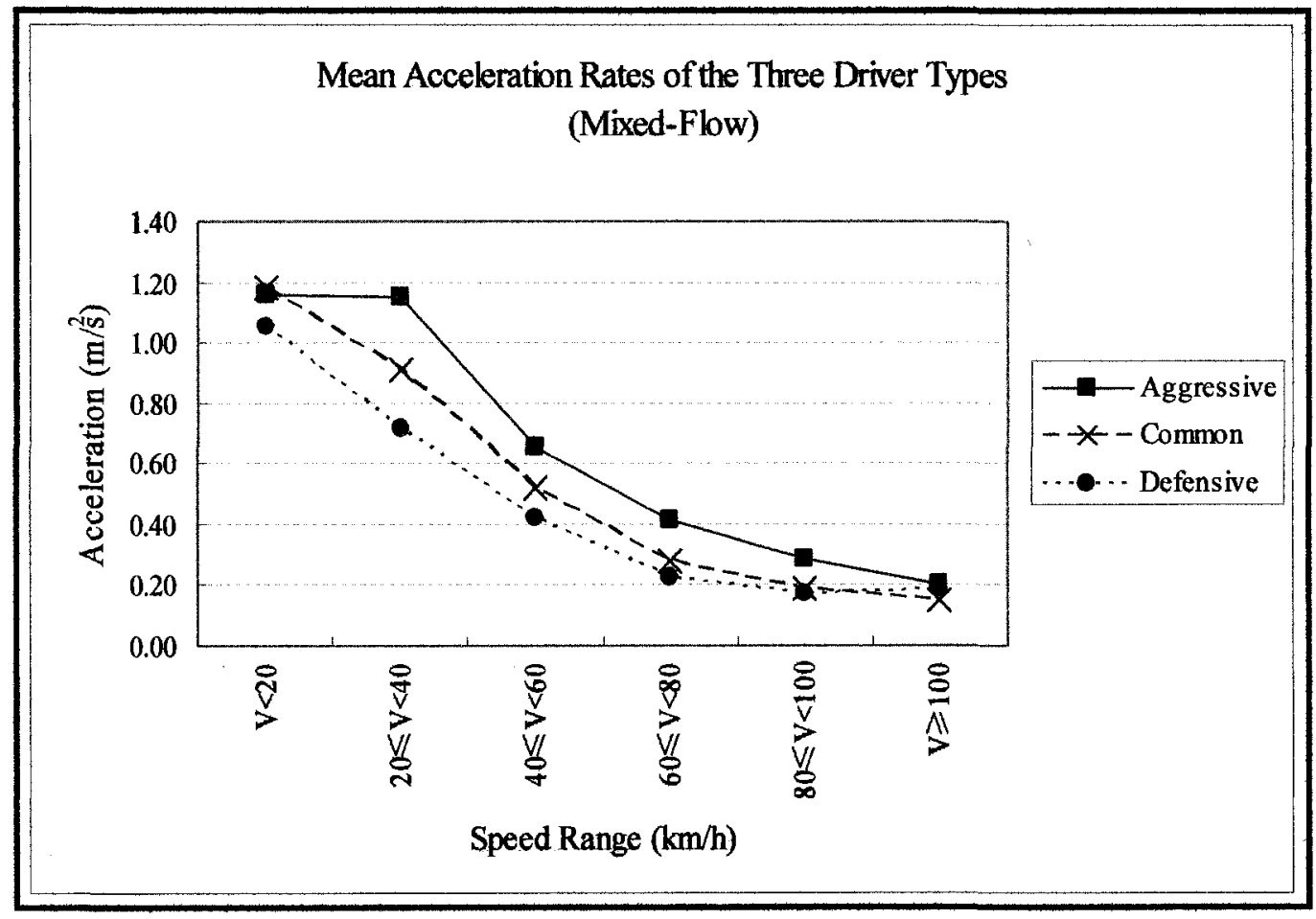

Figure 6-15: Acceleration of the Three Driver Types under Mixed Flow Conditions.

\subsubsection{Comparison of Deceleration Behaviour}

Table 6-15 presents the statistics of deceleration data for the three driver types and corresponding mean deceleration rates are illustrated in Figure 6-16. The figure shows a similar trend for the deceleration behaviour, where the different driver types adopted different deceleration rates. Namely, aggressive drivers generally used harder 
deceleration than the other two types of drivers, whereas defensive drivers were more likely to decelerate the vehicle in a gentler manner compared with aggressive and common drivers. The difference was also more evident in the middle speed ranges between 20 and $80 \mathrm{~km} / \mathrm{h}$.

Table 6-15: Statistics of Deceleration of the Three Driver Types under Mixed Flow Conditions.

\begin{tabular}{|c|c|c|c|c|c|c|c|}
\hline \multirow{2}{*}{$\begin{array}{c}\text { Driver } \\
\text { Type }\end{array}$} & $\begin{array}{c}\text { Deceleration } \\
\text { Parameter } \\
\left(\mathrm{m} / \mathrm{s}^{2}\right)\end{array}$ & \multicolumn{6}{|c|}{ Speed Range $(\mathrm{km} / \mathrm{h})$} \\
\cline { 3 - 8 } Aggressive & Mean & 1.085 & 1.345 & 0.679 & 0.441 & 0.313 & 0.248 \\
\cline { 2 - 8 } & $\mathrm{STD}$ & 0.791 & 0.817 & 0.691 & 0.474 & 0.309 & 0.242 \\
\hline \multirow{2}{*}{ Common } & Mean & 0.989 & 1.183 & 0.608 & 0.298 & 0.180 & 0.161 \\
\cline { 2 - 8 } & $\mathrm{STD}$ & 0.731 & 0.759 & 0.612 & 0.324 & 0.154 & 0.139 \\
\hline \multirow{2}{*}{ Defensive } & Mean & 1.088 & 0.849 & 0.453 & 0.218 & 0.180 & 0.193 \\
\cline { 2 - 8 } & $\mathrm{STD}$ & 0.735 & 0.773 & 0.502 & 0.204 & 0.138 & 0.158 \\
\hline
\end{tabular}

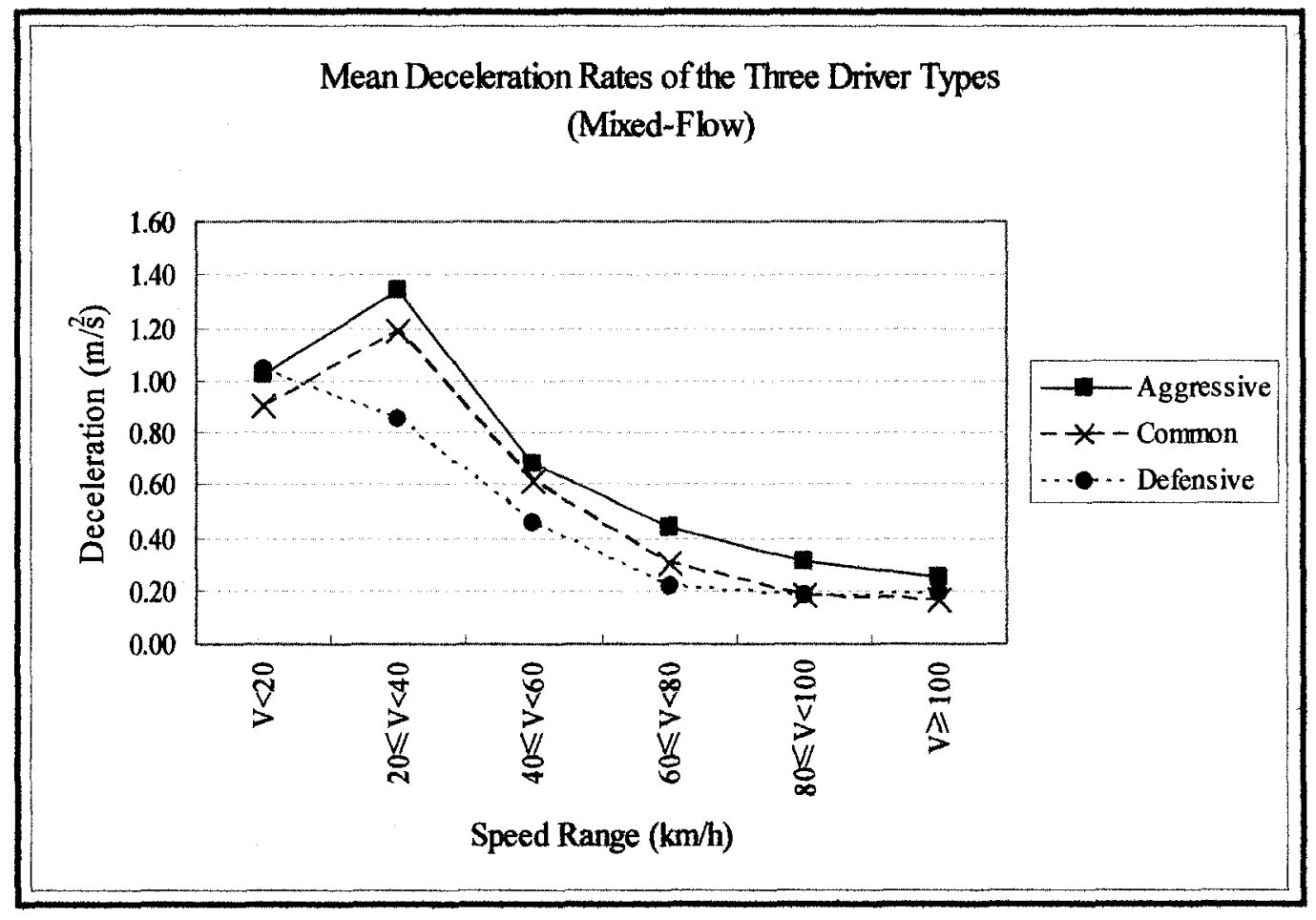

Figure 6-16: Deceleration of the Three Driver Types under Mixed Flow Conditions. 


\section{CHAPTER 7: CONCLUSIONS AND RECOMMENDATIONS}

\subsection{Summary}

This thesis presented a comprehensive study of driver speed behaviour and acceleration/deceleration behaviour on different classes of Canadian roads, using real driving data collected with an instrumented vehicle on a pre-designated test route in Eastern Ontario. The selected test route covered extensive road classes that are often used in drivers' daily travel in Canada. The superior data collection method in this study allowed the capture of vehicle speed and location in an accurate, efficient, and constant manner. Driver speeds and vehicle trajectory were matched to road networks in a GIS environment, and the traffic flow was identified as constrained or unconstrained status. The drivers were categorized into different aggressiveness degrees according to their relative speed performance in the sample. Driver speed behaviour was analyzed corresponding to road classes, traffic flow conditions, posted speed limits, and driver aggressiveness degrees. Driver acceleration and deceleration behaviour were analyzed in relation to driving speed with respect to road classes, traffic flows, and driver aggressiveness degrees. In addition, an approximate framework was developed for identifying a driver's aggressiveness according to the relationship of his/her speed behaviour and the estimated speed behaviour of the general driver population.

\subsection{Findings of This Research}

Based on the analyses presented in the thesis, the key findings can be summarized as follows: 
- Drivers presented different speed behaviour in relation to various road classes of distinct general geometric characteristics and land-use patterns. The generous design of freeway facilities and effective access-control on freeways are more favourable for drivers employing high speeds. In contrast, the frequent intersections in the urban/suburban environment and design at minimum criteria on urban and suburban roads provide fewer chances for drivers to select high speeds.

- In addition, the driver speed behaviour on different road classes is highly dependent on the traffic conditions on these roads. For example, the portion of free-flow speeds on the rural freeway (47.3\%) was almost double of that on the urban freeway $(24.5 \%)$. This fact resulted in a substantially higher average speed on the rural freeway $(107.18 \mathrm{~km} / \mathrm{h})$ than that on the urban freeway $(101.23 \mathrm{~km} / \mathrm{h})$, even though they have the same standards in geometric design and traffic control.

- For the freeways and two-lane rural highways, more than half of the speed observations were above the PSL on each road (58.2\% for Highway 417, 64.2\% for two-lane rural highways, and $74.7 \%$ for Highway 416 ). In contrast, only 6.4 percent of speed observations were above the highest speed limit of $80 \mathrm{~km} / \mathrm{h}$ on urban/suburban roads. The average speeds on freeways and two-lane rural highways were all greater than the corresponding PSL for both mixed-flow and free-flow conditions.

- The general driver population tends to exceed the PSL but keep their speed within $10 \mathrm{~km} / \mathrm{h}$ above the posted speed limit for most of their travel time. Such a 
behaviour may have been influenced by the belief that no speeding ticket would be issued for such a minor violation of speed limits.

- Based on the collected speed data, drivers were classified, in terms of aggressiveness, into three groups. Aggressive drivers usually tried to drive above the speed limits whereas defensive driver preferred to keep their speed below the speed limits. On the other hand, common drivers were more likely to drive at speeds not exceeding speed limits to unreasonable extents. This finding agrees with the research by Fitzpatrick et al. (2003).

- The difference of driver speed choice in relation to the PSL between different driver types was more evident on freeways and two-lane rural highways than on urban/suburban roads. It implies that the distance between successive intersections plays an important role in determining the speed choice of all types of drivers. In other words, the much shorter distance between traffic control devices (stop signs or traffic lights) on urban/suburban roads hinders drivers' ability to drive at speeds exceeding PSL. On the other hand, drivers have better chances to drive as they desire on freeways and rural highways due to the fact that freeways have no at-grade intersections and rural highways have relatively long distances between intersections.

- The average free-flow speeds were generally greater than the corresponding values for non-free-flow conditions. However, the difference between the two flows of speeds become less evident when traffic volumes were low. The situations on two-lane rural highways and Highway 416 were good examples. The explanation for this finding is that the leading vehicle in front could also run 
at high speed in light traffic flow, thus it was not necessarily an impediment for the trailing vehicle, even if the time headway was less than 5 seconds. The drivers are more likely to trail the front vehicle by choice since they have many chances to make a lane change and overtake the front vehicle in these circumstances. The explanation was supported by the video log of driver-frontview for the test runs.

- The acceleration and deceleration behaviour of the driver sample under free-flow conditions were different from that under non-free-flow conditions for the test route. The mean acceleration/deceleration rates in the same speed ranges were generally close to each other for different road classes under free-flow conditions. On the other hand, there was more variation in the drivers' mean acceleration/deceleration rates with the road class under non-free-flow conditions.

- Under free-flow conditions, driver acceleration/deceleration behaviour was found to depend on the instantaneous speeds, especially in low and moderate speed ranges. In these speed ranges, the acceleration/deceleration rates decreased with the increase of speed, but the highest value did not correspond to the state of operation before and after a complete stop (speed lower than $10 \mathrm{~km} / \mathrm{h}$ ). On the other hand, the acceleration/deceleration rates tended to stabilize in the high speed ranges (speed higher than $80 \mathrm{~km} / \mathrm{h}$ ).

- The difference of acceleration and deceleration behaviour between different types of drivers was evident. The classified aggressive drivers generally adopted 
the highest acceleration and deceleration rates and those classified as defensive adopted the softest rates.

\subsection{Contributions of This Research}

This research contributes in a number of ways to the advancement of transportation safety analysis. First, it drew a quantitative picture of driver speed and acceleration/deceleration behaviours on Canadian roads. This provides diverse jurisdictions a better understanding of driver behaviour on our roads and, thus, a reference for future traffic management and highway design. Second, the research successfully identified drivers with different degrees of aggressiveness and proposed a framework for recognizing different types of drivers according to their speed behaviour. It is expected that early detection and intervention techniques for problem behaviour could be developed accordingly to eliminate potential accidents and that positive changes in the behaviour of individual drivers could also be actualized through a public education campaign. Third, this research relied on instrumented vehicle technologies to collect accurate and detailed data on driver behaviour. The technologies are found to be promising and reliable for future application to efficiently detect driver behaviour and exposure.

\subsection{Recommendations for Future Study}

Recommendations for future studies in this area follow:

- The sample size in this study is relatively small; the results should not be simply generalized to any locality without further validation. 
- Using the data collected in the experiment, further studies could address the issue of driver acceleration and deceleration behaviour at specific locations, such as intersections.

- Drivers usually vary their speed and acceleration when they make a lane change for overtaking other vehicles or driving in/out of the speed-change-lane at freeway interchanges. More efforts could be directed at the study of driver speed and acceleration behaviour related to lane-change and passing manoeuvres.

- Due to the lack of extra data, the results of the framework for evaluating driver's aggressiveness in this research was not validated using other data. It is suggested that the framework will be improved based on a larger sample size from Netistix pilot project, in which real driving data of a large number of drivers are to be collected using the VIU.

- Driver speed and acceleration behaviour could be affected by many other factors such as driver age, gender and the urgency to arrive to their destination. Studies in the future could focus on factors affecting speed. 


\section{REFERENCES}

Akcelik, R. and Biggs, D. C. 1987. Acceleration Profile Models for Vehicles in Road Traffic. Transportation Science, Vol. 21, No. 1, pp. 36-54.

American Association of State Highway and Transportation Officials. 2001. A Policy on Geometric Design of Highways and Streets, Washington, D.C.

American Association of State Highway and Transportation Officials. 2004. A Policy on Geometric Design of Highways and Streets, Washington, D.C.

Bennett, C. R. and Dunn, R. C. M. 1995. Driver Deceleration Behavior on a Freeway in New Zealand. Transportation Research Record 1510, TRB, Transportation Research Council, Washington, D.C., pp.70-75.

Burden, R. L. and Faires, J. D. 1997. Numerical Analysis, Sixth Edition, Brooks/Cole Publishing Company, ISBN 0-534-95532-0.

Chakroborty, P., Agrawal, S., and Vasishtha, K. 2004. Microscopic Modeling of Driver Behavior in Uninterrupted Traffic Flow. Journal of Transportation Engineering, ASCE, Vol. 130(4), pp. 438-451.

EI-Basha, R. H. S., Hassan, Y. and Sayed, T. A. 2007. Modeling Freeway Diverging Behavior on Deceleration Lanes. Transportation Research Board $86^{\text {th }}$ Annual Meeting, Washington, D.C., CD-ROM, Paper No, 07-2041.

EI-Shawarby, I., Rakha, H., Inman, V. W. and Davis, G. W. 2007. Evaluation of Driver Deceleration Behavior at Signalized Intersections. Transportation Research Board $86^{\text {th }}$ Annual Meeting, Washington, D.C., CD-ROM, Paper No. 07-1465. 
Ericsson, E. 2000. Variability in Urban Driving Patterns. Transportation Research, Part D, Vol. $5(5)$, pp337-354.

European Transport Safety Council (ETSC). 1995. Reducing Traffic Injuries Resulting from Excess and Inappropriate Speed. Brussels.

Federal Highway Administration, U.S. Department of Transportation. 2003. Manual on Uniform Traffic Control Devices for Streets and Highways - 2003 Edition. Washington, DC.

Feng, C. 2001. Synthesis of Studies on Speed and Safety. Transportation Research Record 1779, Journal of Transportation Research Board, TRB, Transportation Research Council, Washington, D.C., pp.86-92.

Finch, D.J., Kompfner, P., Lockwood, C. R., and Maycock, G. 1994. Speed, speed limits and accidents. Project Report 58. Crowthorne: Transport Research Laboratory (TRL). Cited in ETSC (1995).

Fitzpatric, K., Carlson, P., Brewer, M., and Wooldridge, M. 2001. Design Factors That Affect Driver Speed on Suburban Streets. Transportation Research Record 1751, Journal of Transportation Research Board, TRB, Transportation Research Council, Washington, D.C., pp.18-25.

Fitzpatrick, K., Carlson, P., Brewer, M., Wooldridge, M., and Miaou, S. 2003. Design Speed, Operating Speed, and Posted Speed Practices. National Cooperative Highway Research Program, Report 504, Transportation Research Board. 
Fitzpatrick, K., Shamburger, B., Krammes, R. A., and Fambro, D. B. 1997. Operating Speed on Suburban Arterial Curves. Transportation Research Record 1579, TRB, Transportation Research Council, Washington, D.C., pp.89-96.

Fowles, R. and Loeb, P. D. 1989. Speeding, Coordination, and the 55-MPH Limit: Comment. The American Economic Review, Vol. 79(4), pp 916-21.

Garber, N J and Gadiraju, R. 1989. Factors Affecting Speed Variance and Its Influence on Accidents. Transportation Research Record 1213, TRB, Transportation Research Council, Washington, D.C., pp.64-71.

Gates, T. J., Noyce, D. A., and Laracuente, L. 2007. Analysis of Dilemma Zone Driver Behavior at Signalized Intersections. Transportation Research Board $86^{\text {th }}$ Annual Meeting, Washington, D.C., CD-ROM, Paper No. 07-3351.

Gattis, J. L. and Watts, A. 1999. Urban Street Speed Related to Width and Functional Class. Journal of Transportation Engineering, Vol. 125, No. 3. pp193-200.

Giles, M. J. 2003. Driver Speed Compliance in Western Australia: A Multivariate Analysis. Transport Policy (11), Elsevier Ltd., pp.227-235.

Haas, R., Inman, V, Dixson, A., and Warren, D. 2004. Use of Intelligent Transportation System Data to Determine Driver Deceleration and Acceleration Behavior. Transportation Research Record 1899, Journal of Transportation Research Board, TRB, Transportation Research Council, Washington, D.C., pp.3-10.

Hassan, Y. 2004. Highway Design Consistency: Refining the State of Knowledge and Practice. Transportation Research Record 1881, Journal of Transportation Research Board, TRB, National Research Council, Washington, D. C., pp. 63-71. 
Institute of Transportation Engineers. 1982. Transportation and Traffic Engineering Hand Book, $2^{\text {nd }}$ edition. Prentice-Hall, Englewood Cliffs, N.J. Cited in Mousa (2002).

Institute of Transportation Engineers. 1992. Traffic Engineering Handbook, $4^{\text {th }}$ Edition. Prentice-Hall, Englewood Cliffs, N.J.

Insurance Institute of Highway Safety (IIHS). 2007. $Q \&$ A: Speed and Speed Limits. http://www.iihs.org/research/qanda/speed_limits.html. Accessed on April 17, 2007.

Jun, J., Ogle, J., and Guensler, R. 2007. Relationship between Crash Involvement and Temporal-Spatial Driving Behavior Activity Patterns Using GPS Instrumented Vehicle Data. Transportation Research Board $86^{\text {th }}$ Annual Meeting, Washington, D.C., CD-ROM, Paper No. 07-3059.

Kanellaidis, G. 1995. Factors Affecting Drivers' Choice of Speed on Roadway Curves. Journal of Safety Research, National Safety Council and Elsevier Science Ltd, Vol. 26(1), pp. 49-56.

Kanellaidis, G., Golias, J., and Efstathiadis, S. 1990. Driver's Speed Behavior on Rural Road Curves. Traffic Engineering \& Control. Vol. 31, No.7, pp. 414-415.

Kloeden, CN., McLean, AJ., Moore, VM. and Ponte, G. 1997. Reanalysis of Traveling Speed and the Risk of Crash Involvement in Adelaide South Australia. CR 207. Australian Transport Safety Bureau. Canberra, Australia. http://raru.adelaide.edu.au/speed. Accessed on April 20, 2007.

Krammes, R. A., 2000. Design Speed and Operating Speed in Rural Highway Alignment Design. Transportation Research Record 1701, Journal of Transportation Research Board, TRB, Transportation Research Council, Washington, D.C., pp.68-75. 
Lave, Charles A.1985. Speeding, Coordination, and the 55 MPH Limit. The American Economic Review, Vol. 75(5), pp 1159-64.

Leica Geosystems Inc. 2001. SKI-Pro GPS Software User Manual, Version 2.0. Heerbrugg, Switzerland.

Levy, D. T. and Asch, P. 1989. Speeding, Coordination, and the 55-MPH Limit: Comment. The American Economic Review, Vol. 79(4), pp 913-15.

Liu G. X. and Popoff, A. 1997. Provincial-Wide Travel Speed and Traffic Safety Study in Saskatchewan. Transportation Research Record 1595, TRB, Transportation Research Council, Washington, D.C., pp.8-13.

Liu, Y., Change, G. L., Tao, R., Hicks, T. and Tabacek, E. 2007. Empirical Observations of Dynamic Dilemma Zones at Signalized Intersections. Transportation Research Board $86^{\text {th }}$ Annual Meeting, Washington, D.C., CD-ROM, Paper No.07-1658.

Long, G. 2000. Acceleration Characteristics of Starting Vehicles. Transportation Research Record 1737, Journal of Transportation Research Board, TRB, Transportation Research Council, Washington, D.C., pp.58-70.

Mousa, R. M. 2002. Analysis and Modeling of Measured Delays at Isolated Signalized Intersections. Journal of Transportation Engineering, ASCE, Vol. 128(4), pp.347-354.

National Highway Traffic Safety Administration (NHTSA). 1998. National Survey of Speeding and Other Unsafe Driving Actions. U.S. Department of Transportation, Washington, D.C. $\quad$ http://www.nhtsa.dot.gov/people/injury/aggressive/unsafe/, Accessed on April 10, 2007. 
National Highway Traffic Safety Administration (NHTSA). 2002. National Survey of Speeding and Other Unsafe Driving Attitudes and behaviour: 2002. U.S. Department of Transportation, Washington, D.C.

National Highway Traffic Safety Administration (NHTSA). 2006a. Traffic Safety Facts 2005: Overview (DOT Publication No. DOT HS 810 623). National Center for Statistics and Analysis, Washington, D.C.

National Highway Traffic Safety Administration (NHTSA). 2006b. Traffic Safety Facts 2005: Speeding (DOT Publication No. DOT HS 810 629). National Center for Statistics and Analysis, Washington, D.C.

Nesamani, K. S., Subramanian, K. P., Jayakrishnan, R. and McNally, M. G.. 2006. Factors Affecting Speed of Motor Vehicles on Urban Arterials: Case of Chennai, India. Transportation Research Board $85^{\text {th }}$ Annual Meeting. Transportation Research Council, Washington, D.C., CD-ROM, Paper No. 06-2984.

Nie, B. 2006. Effect of Horizontal Alignment on Driver Speed Behaviour on Different Road Classifications. M.A.Sc Thesis. Carleton University, Ottawa, Ontario, Canada.

Ogle, J. 2005. Quantitative Assessment of Driver Speeding Behavior Using Instrumented Vehicles, Doctoral Dissertation. The Georgia Institute of Technology, Atlanta.

Ontario Ministry of Transportation (MTO). 2004. Provincial Highways Traffic Volumes 1998-2004. Toronto, Ontario.

Ontario Ministry of Transportation (MTO). 2006. Road Safety Program Office. Ontario Road Safety Annual Report (ORSAR) 2004. Toronto, Ontario. 
Ottesen, J. L. and Krammes, R. A. 2000. Speed-Profile Model for a Design-Consistency Evaluation Procedure in the United States. Transportation Research Record 1701, Journal of Transportation Research Board, TRB, National Research Council, Washington, D. C. pp76-85.

Pasanen, E. and Salmivaara, H. 1993. Driving Speeds and Pedestrian Safety in the City of Helsinki. Traffic Engineering \& Control. Vol. 34, No. 6, pp.308-310.

Raub, R. A., Wark, R. I., and Lucke, R. E. 2002. Seeking a Reduction in Aggressive Driving Through Different Strategies. Transportation Research Board $81^{\text {st }}$ Annual Meeting, Washington, D.C. CD-ROM, Paper No. 02-2786.

Rodriguez, R. J. 1990. Speed, Speed Dispersion, and the Highway Fatality Rate. Southern Economic Journal, Vol. 57, No.2. pp349-356.

Shinar, D. 1998. Aggressive Driving: The Contribution of the Drivers and the Situation. Transportation Research, 1F(2), Elsevier Ltd., pp137-160.

Sin, H. G. 2001. Field Evaluation Methodology for Quantifying Network-Wide Efficiency Energy, Emission, and Safety Impacts of Operational-Level Transportation Projects. Doctoral Dissertation. Virginia Polytechnic Institute and State University, Blacksburg, Virginia.

Snyder, D. 1989. Speeding, Coordination, and the 55-MPH Limit: Comment. The American Economic Review, Vol. 79(4), pp 922-25.

Solomon, D. 1964. Accidents on Main Rural Highways Related to Speed, Driver, and Vehicle. Bureau of Public Roads, U.S. Department of Commerce. Cited in Liu and Popoff (1997). 
Transport Canada. 2004. Road Safety Program. Road Safety in Canada-An Overview. Ottawa, Ontario.

Transport Canada. 2006. Road Safety and Motor Vehicle Regulation Directorate. Road Safety in Canada-2003. Ottawa, Ontario.

Transport Canada. 2007a. 2005 Annual Report on Road Safety Vision 2010. Ottawa, Ontario. http://www.tc.gc.ca/roadsafety/vision/2005/pdf/rsv2005se.pdf. Accessed on July 25, 2007.

Transport Canada. 2007b. Canadian Motor Vehicle Traffic Collision Statistics: 2005. Ottawa, Ontario. http://www.tc.gc.ca/roadsafety/tp/tp3322/2005/page1.htm. Accessed on June 05, 2007.

Transportation Association of Canada (TAC). 1999. Geometric Design Guide for Canadian Roads. Ottawa, Ontario.

TRB. 2001. Special Report 260: Strategic Highway Research: Saving Lives, Reducing Congestion, Improving Quality of Life. National Research Council, Washington, D.C.

Van Aerde, M. and Yagar, S. 1983. Volume Effects on Speeds of 2-Lane Highways in Ontario. Transportation Research, 17A(4), Pergamon Press, pp.301-313.

Wang, J., Dixon, K. K., Li, H., and Ogle, J. 2004. Normal Acceleration Behavior of Passenger Vehicles Starting from Rest at All-Way Stop-Controlled Intersections. Transportation Research Record 1883, Journal of Transportation Research Board, TRB, Transportation Research Council, Washington, D.C., pp.158-166. 
Wortman, R. H. and Matthias, J. S. 1983. Evaluation of Driver Behavior at Signalized Intersections. Transportation Research Record 904, TRB, Transportation Research Council, Washington, D.C., pp.10-20.

Yagar, S. and Van Aerde, M. 1983. Geometric and Environmental Effects on Speeds of 2-Lane Highways. Transportation Research, 17A(4), Pergamon Press, pp.315-325.

Zaal, D. 1994. Traffic Law Enforcement: A Review of the Literature, Report No. 53. Federal Office of Road Safety, Canberra. Australia. 


\section{APPENDIX A: SPEED STATISTICS OF INDIVIDUAL DRIVERS FOR DIFFERENT ROAD CLASSES}


Table A - 1: Speed Statistics of Individual Drivers on Urban Freeway under Mixed Flow Conditions.

\begin{tabular}{|c|c|c|c|c|}
\hline \multirow{2}{*}{ Driver ID } & \multicolumn{4}{|c|}{ Speed Parameter $(\mathrm{km} / \mathrm{h})$} \\
\cline { 2 - 5 } & Minimum & Maximum & Mean & Std. Deviation \\
\hline Driver 02 & 85 & 121 & 103.79 & 8.56 \\
\hline Driver 03 & 75 & 113 & 97.01 & 10.37 \\
\hline Driver 04 & 75 & 107 & 96.86 & 4.97 \\
\hline Driver 05 & 74 & 123 & 98.87 & 8.48 \\
\hline Driver 06 & 78 & 109 & 99.02 & 5.49 \\
\hline Driver 08 & 72 & 104 & 94.90 & 5.09 \\
\hline Driver 09 & 81 & 120 & 109.96 & 5.12 \\
\hline Driver 10 & 77 & 114 & 97.72 & 5.73 \\
\hline Driver 11 & 59 & 117 & 103.15 & 7.06 \\
\hline Driver 12 & 88 & 134 & 116.00 & 8.28 \\
\hline Driver 13 & 77 & 118 & 101.79 & 7.45 \\
\hline Driver 14 & 83 & 125 & 105.10 & 7.26 \\
\hline Driver 15 & 79 & 124 & 109.35 & 5.71 \\
\hline Driver 16 & 93 & 137 & 111.85 & 8.17 \\
\hline Driver 17 & 74 & 111 & 95.46 & 7.40 \\
\hline Driver 18 & 70 & 131 & 110.28 & 11.19 \\
\hline Driver 19 & 61 & 109 & 91.14 & 7.78 \\
\hline Driver 20 & 80 & 124 & 104.39 & 8.72 \\
\hline Driver 21 & 84 & 112 & 102.04 & 4.67 \\
\hline Driver 22 & 34 & 116 & 88.51 & 18.30 \\
\hline Driver 23 & 60 & 101 & 89.42 & 5.23 \\
\hline Driver 24 & 70 & 111 & 101.03 & 5.08 \\
\hline Driver 25 & 77 & 118 & 100.84 & 6.81 \\
\hline Driver 26 & 74 & 123 & 107.35 & 6.98 \\
\hline Driver 31 & 74 & 118 & 102.85 & 6.24 \\
\hline Driver 32 & 88 & 124 & 104.87 & 6.32 \\
\hline Total & 34 & 137 & 101.23 & 10.43 \\
\hline
\end{tabular}


Table A - 2: Speed Statistics of Individual Drivers on Urban Freeway under Free-Flow Conditions.

\begin{tabular}{|c|c|c|c|c|}
\hline \multirow{2}{*}{ Driver ID } & \multicolumn{4}{|c|}{ Speed Parameter $(\mathrm{km} / \mathrm{h})$} \\
\cline { 2 - 5 } & Minimum & Maximum & Mean & Std. Deviation \\
\hline Driver 02 & 94 & 121 & 108.81 & 5.55 \\
\hline Driver 03 & 81 & 112 & 101.17 & 6.48 \\
\hline Driver 04 & 75 & 104 & 96.23 & 5.38 \\
\hline Driver 05 & 91 & 123 & 105.04 & 6.66 \\
\hline Driver 06 & 91 & 109 & 102.49 & 2.90 \\
\hline Driver 08 & 88 & 102 & 98.47 & 2.77 \\
\hline Driver 09 & 104 & 120 & 112.95 & 3.27 \\
\hline Driver 10 & 94 & 107 & 100.01 & 4.04 \\
\hline Driver 11 & 104 & 114 & 107.60 & 2.16 \\
\hline Driver 12 & 108 & 132 & 118.94 & 6.64 \\
\hline Driver 13 & 88 & 114 & 102.62 & 6.86 \\
\hline Driver 14 & 106 & 123 & 112.72 & 4.78 \\
\hline Driver 15 & 98 & 124 & 111.23 & 5.04 \\
\hline Driver 16 & 94 & 137 & 113.70 & 12.68 \\
\hline Driver 17 & 87 & 107 & 98.73 & 4.36 \\
\hline Driver 18 & 97 & 131 & 114.73 & 7.91 \\
\hline Driver 19 & 87 & 102 & 96.34 & 3.75 \\
\hline Driver 20 & 86 & 122 & 107.49 & 7.60 \\
\hline Driver 21 & 94 & 112 & 104.92 & 3.46 \\
\hline Driver 22 & 75 & 108 & 100.56 & 6.19 \\
\hline Driver 23 & 60 & 97 & 88.46 & 4.97 \\
\hline Driver 24 & 95 & 111 & 103.55 & 4.21 \\
\hline Driver 25 & 87 & 113 & 101.42 & 5.45 \\
\hline Driver 26 & 77 & 118 & 106.98 & 9.80 \\
\hline Driver 31 & 102 & 115 & 107.83 & 2.96 \\
\hline Driver 32 & 98 & 115 & 108.86 & 5.01 \\
\hline Total & 60 & 137 & 104.32 & 9.31 \\
\hline
\end{tabular}


Table A - 3: Speed Statistics of Individual Drivers on Urban Freeway under Non-FreeFlow Conditions.

\begin{tabular}{|c|c|c|c|c|}
\hline \multirow{2}{*}{ Driver ID } & \multicolumn{4}{|c|}{ Speed Parameter $(\mathrm{km} / \mathrm{h})$} \\
\hline & Minimum & Maximum & Mean & Std. Deviation \\
\hline Driver 02 & 85 & 118 & 97.88 & 7.67 \\
\hline Driver 03 & 75 & 113 & 95.92 & 10.91 \\
\hline Driver 04 & 81 & 107 & 97.00 & 4.88 \\
\hline Driver 05 & 74 & 114 & 96.08 & 7.70 \\
\hline Driver 06 & 78 & 107 & 97.46 & 5.66 \\
\hline Driver 08 & 72 & 104 & 94.57 & 5.13 \\
\hline Driver 09 & 81 & 118 & 108.32 & 5.21 \\
\hline Driver 10 & 77 & 114 & 97.42 & 5.86 \\
\hline Driver 11 & 59 & 117 & 102.39 & 7.32 \\
\hline Driver 12 & 88 & 134 & 115.66 & 8.39 \\
\hline Driver 13 & 77 & 118 & 101.54 & 7.61 \\
\hline Driver 14 & 83 & 125 & 104.72 & 7.16 \\
\hline Driver 15 & 79 & 120 & 107.87 & 5.77 \\
\hline Driver 16 & 93 & 129 & 111.56 & 7.16 \\
\hline Driver 17 & 74 & 111 & 94.06 & 7.98 \\
\hline Driver 18 & 70 & 128 & 106.81 & 12.11 \\
\hline Driver 19 & 61 & 109 & 90.55 & 7.90 \\
\hline Driver 20 & 80 & 124 & 102.95 & 8.83 \\
\hline Driver 21 & 84 & 111 & 100.49 & 4.50 \\
\hline Driver 22 & 34 & 116 & 86.00 & 18.98 \\
\hline Driver 23 & 72 & 101 & 90.58 & 5.30 \\
\hline Driver 24 & 70 & 111 & 100.58 & 5.09 \\
\hline Driver 25 & 77 & 118 & 100.55 & 7.38 \\
\hline Driver 26 & 74 & 123 & 107.41 & 6.34 \\
\hline Driver 31 & 74 & 118 & 102.19 & 6.27 \\
\hline Driver 32 & 88 & 124 & 104.01 & 6.25 \\
\hline Total & 34 & 134 & 100.23 & 10.58 \\
\hline
\end{tabular}


Table A - 4: Speed Statistics of Individual Drivers on Two-Lane Rural Highways under Mixed Flow Conditions.

\begin{tabular}{|c|c|c|c|c|}
\hline \multirow{2}{*}{ Driver ID } & \multicolumn{4}{|c|}{ Speed Parameter $(\mathrm{km} / \mathrm{h})$} \\
\hline & Minimum & Maximum & Mean & Std. Deviation \\
\hline Driver 02 & 0 & 107 & 82.16 & 15.81 \\
\hline Driver 03 & 0 & 94 & 77.64 & 13.63 \\
\hline Driver 04 & 0 & 99 & 79.56 & 15.88 \\
\hline Driver 05 & 0 & 99 & 79.95 & 16.04 \\
\hline Driver 06 & 0 & 95 & 80.98 & 14.29 \\
\hline Driver 08 & 0 & 100 & 79.81 & 16.12 \\
\hline Driver 09 & 0 & 112 & 87.65 & 17.11 \\
\hline Driver 10 & 0 & 101 & 79.38 & 15.91 \\
\hline Driver 11 & 0 & 96 & 78.64 & 14.54 \\
\hline Driver 12 & 0 & 133 & 94.49 & 23.61 \\
\hline Driver 13 & 0 & 133 & 96.86 & 22.40 \\
\hline Driver 14 & 0 & 101 & 81.41 & 14.91 \\
\hline Driver 15 & 0 & 103 & 82.49 & 16.58 \\
\hline Driver 16 & 0 & 119 & 87.61 & 17.42 \\
\hline Driver 17 & 0 & 98 & 67.80 & 13.73 \\
\hline Driver 18 & 0 & 123 & 87.72 & 21.55 \\
\hline Driver 19 & 0 & 98 & 73.40 & 17.63 \\
\hline Driver 20 & 0 & 114 & 86.53 & 18.52 \\
\hline Driver 21 & 0 & 99 & 83.05 & 15.67 \\
\hline Driver 22 & 0 & 110 & 80.79 & 14.55 \\
\hline Driver 23 & 0 & 94 & 72.45 & 12.20 \\
\hline Driver 24 & 0 & 93 & 73.85 & 13.30 \\
\hline Driver 25 & 0 & 98 & 68.46 & 16.85 \\
\hline Driver 26 & 0 & 115 & 86.96 & 17.01 \\
\hline Driver 31 & 0 & 102 & 80.02 & 17.79 \\
\hline Driver 32 & 0 & 121 & 91.39 & 23.04 \\
\hline Total & 0 & 133 & 80.98 & 18.23 \\
\hline
\end{tabular}


Table A - 5: Speed Statistics of Individual Drivers on Two-Lane Rural Highways under Free-Flow Conditions.

\begin{tabular}{|c|c|c|c|c|}
\hline \multirow{2}{*}{ Driver ID } & \multicolumn{4}{|c|}{ Speed Parameter $(\mathrm{km} / \mathrm{h})$} \\
\hline & Minimum & Maximum & Mean & Std. Deviation \\
\hline Driver 02 & 0 & 107 & 83.21 & 14.69 \\
\hline Driver 03 & 0 & 94 & 78.16 & 12.14 \\
\hline Driver 04 & 0 & 99 & 80.56 & 14.76 \\
\hline Driver 05 & 0 & 99 & 79.99 & 15.76 \\
\hline Driver 06 & 0 & 94 & 80.79 & 14.37 \\
\hline Driver 08 & 0 & 100 & 77.94 & 17.56 \\
\hline Driver 09 & 0 & 110 & 86.48 & 17.26 \\
\hline Driver 10 & 0 & 101 & 78.03 & 15.94 \\
\hline Driver 11 & 0 & 95 & 78.58 & 14.53 \\
\hline Driver 12 & 0 & 133 & 94.21 & 25.69 \\
\hline Driver 13 & 0 & 133 & 103.63 & 24.26 \\
\hline Driver 14 & 0 & 101 & 81.53 & 12.21 \\
\hline Driver 15 & 0 & 103 & 82.61 & 16.59 \\
\hline Driver 16 & 0 & 119 & 87.83 & 17.08 \\
\hline Driver 17 & 0 & 98 & 68.13 & 13.47 \\
\hline Driver 18 & 0 & 123 & 92.04 & 21.36 \\
\hline Driver 19 & 0 & 98 & 76.16 & 15.96 \\
\hline Driver 20 & 0 & 114 & 89.00 & 18.06 \\
\hline Driver 21 & 0 & 99 & 83.57 & 14.56 \\
\hline Driver 22 & 0 & 110 & 79.30 & 14.20 \\
\hline Driver 23 & 0 & 94 & 72.41 & 12.22 \\
\hline Driver 24 & 0 & 93 & 73.89 & 13.08 \\
\hline Driver 25 & 0 & 93 & 73.54 & 10.23 \\
\hline Driver 26 & 0 & 115 & 89.18 & 24.11 \\
\hline Driver 31 & 0 & 102 & 83.61 & 14.70 \\
\hline Driver 32 & 0 & 121 & 97.65 & 19.60 \\
\hline Total & 0 & 133 & 81.29 & 17.59 \\
\hline
\end{tabular}


Table A - 6: Speed Statistics of Individual Drivers on Two-Lane Rural Highways under Non-Free-Flow Conditions.

\begin{tabular}{|c|c|c|c|c|}
\hline \multirow{2}{*}{ Driver ID } & \multicolumn{4}{|c|}{ Speed Parameter $(\mathrm{km} / \mathrm{h})$} \\
\hline & Minimum & Maximum & Mean & Std. Deviation \\
\hline Driver 02 & 0 & 92 & 75.17 & 20.56 \\
\hline Driver 03 & 0 & 90 & 72.10 & 23.83 \\
\hline Driver 04 & 0 & 94 & 58.75 & 22.69 \\
\hline Driver 05 & 0 & 94 & 79.38 & 19.40 \\
\hline Driver 06 & 0 & 95 & 90.11 & 2.99 \\
\hline Driver 08 & 0 & 95 & 83.82 & 11.53 \\
\hline Driver 09 & 0 & 112 & 91.03 & 16.25 \\
\hline Driver 10 & 0 & 99 & 90.88 & 9.97 \\
\hline Driver 11 & 0 & 96 & 82.55 & 15.28 \\
\hline Driver 12 & 0 & 126 & 95.37 & 15.42 \\
\hline Driver 13 & 0 & 122 & 89.21 & 17.14 \\
\hline Driver 14 & 0 & 100 & 81.07 & 21.16 \\
\hline Driver 15 & 0 & 102 & 82.32 & 16.58 \\
\hline Driver 16 & 0 & 112 & 86.77 & 18.69 \\
\hline Driver 17 & 0 & 86 & 65.27 & 15.34 \\
\hline Driver 18 & 0 & 113 & 78.51 & 18.92 \\
\hline Driver 19 & 0 & 92 & 69.18 & 19.16 \\
\hline Driver 20 & 0 & 105 & 82.45 & 18.57 \\
\hline Driver 21 & 0 & 98 & 66.70 & 32.02 \\
\hline Driver 22 & 0 & 107 & 84.03 & 14.81 \\
\hline Driver 23 & 0 & 83 & 80.50 & 1.31 \\
\hline Driver 24 & 0 & 89 & 72.96 & 17.93 \\
\hline Driver 25 & 0 & 98 & 55.37 & 22.55 \\
\hline Driver 26 & 0 & 108 & 85.63 & 10.45 \\
\hline Driver 31 & 0 & 102 & 69.52 & 21.51 \\
\hline Driver 32 & 0 & 119 & 80.66 & 24.51 \\
\hline Total & 0 & 126 & 79.84 & 20.37 \\
\hline
\end{tabular}


Table A - 7: Speed Statistics of Individual Drivers on Rural Freeway under Mixed Flow Conditions.

\begin{tabular}{|c|c|c|c|c|}
\hline \multirow{2}{*}{ Driver ID } & \multicolumn{4}{|c|}{ Speed Parameter $(\mathrm{km} / \mathrm{h})$} \\
\hline & Minimum & Maximum & Mean & Std. Deviation \\
\hline Driver 02 & 73 & 119 & 107.34 & 5.03 \\
\hline Driver 03 & 73 & 115 & 107.07 & 4.64 \\
\hline Driver 04 & 69 & 120 & 106.02 & 10.26 \\
\hline Driver 05 & 74 & 112 & 103.21 & 4.18 \\
\hline Driver 06 & 65 & 110 & 103.37 & 4.67 \\
\hline Driver 08 & 72 & 112 & 101.13 & 6.39 \\
\hline Driver 09 & 60 & 120 & 113.55 & 7.97 \\
\hline Driver 10 & 73 & 119 & 104.57 & 5.49 \\
\hline Driver 11 & 74 & 112 & 105.82 & 4.07 \\
\hline Driver 12 & 67 & 132 & 123.10 & 9.34 \\
\hline Driver 13 & 78 & 146 & 129.62 & 10.78 \\
\hline Driver 14 & 67 & 127 & 109.27 & 7.83 \\
\hline Driver 15 & 64 & 116 & 108.38 & 6.01 \\
\hline Driver 16 & 76 & 146 & 116.78 & 12.10 \\
\hline Driver 17 & 66 & 104 & 95.23 & 4.61 \\
\hline Driver 18 & 69 & 137 & 122.47 & 8.72 \\
\hline Driver 19 & 58 & 104 & 96.82 & 5.67 \\
\hline Driver 20 & 67 & 120 & 108.92 & 6.43 \\
\hline Driver 21 & 75 & 115 & 106.89 & 4.27 \\
\hline Driver 22 & 83 & 115 & 108.08 & 3.93 \\
\hline Driver 23 & 65 & 102 & 91.72 & 4.83 \\
\hline Driver 24 & 72 & 105 & 95.82 & 3.81 \\
\hline Driver 25 & 69 & 104 & 91.02 & 5.87 \\
\hline Driver 26 & 75 & 139 & 124.51 & 8.03 \\
\hline Driver 31 & 72 & 117 & 105.21 & 5.40 \\
\hline Driver 32 & 84 & 134 & 124.78 & 7.35 \\
\hline Total & 58 & 146 & 107.18 & 12.02 \\
\hline
\end{tabular}


Table A - 8: Speed Statistics of Individual Drivers on Rural Freeway under Free-Flow Conditions.

\begin{tabular}{|c|c|c|c|c|}
\hline \multirow{2}{*}{ Driver ID } & \multicolumn{4}{|c|}{ Speed Parameter $(\mathrm{km} / \mathrm{h})$} \\
\hline & Minimum & Maximum & Mean & Std. Deviation \\
\hline Driver 02 & 73 & 119 & 109.33 & 8.22 \\
\hline Driver 03 & 101 & 115 & 107.22 & 3.09 \\
\hline Driver 04 & 84 & 120 & 107.55 & 8.55 \\
\hline Driver 05 & 98 & 112 & 105.07 & 3.32 \\
\hline Driver 06 & 65 & 110 & 103.35 & 6.63 \\
\hline Driver 08 & 98 & 112 & 104.08 & 3.19 \\
\hline Driver 09 & 60 & 120 & 113.60 & 8.28 \\
\hline Driver 10 & 95 & 118 & 103.72 & 6.13 \\
\hline Driver 11 & 99 & 112 & 106.62 & 2.79 \\
\hline Driver 12 & 67 & 132 & 122.84 & 9.91 \\
\hline Driver 13 & 78 & 146 & 133.12 & 10.56 \\
\hline Driver 14 & 67 & 124 & 107.41 & 12.53 \\
\hline Driver 15 & 64 & 115 & 106.52 & 9.27 \\
\hline Driver 16 & 98 & 143 & 117.83 & 11.04 \\
\hline Driver 17 & 66 & 104 & 94.83 & 5.00 \\
\hline Driver 18 & 69 & 137 & 122.25 & 11.05 \\
\hline Driver 19 & 58 & 103 & 96.35 & 6.66 \\
\hline Driver 20 & 67 & 120 & 109.98 & 11.43 \\
\hline Driver 21 & 75 & 113 & 106.86 & 4.14 \\
\hline Driver 23 & 65 & 102 & 90.95 & 4.85 \\
\hline Driver 24 & 72 & 103 & 94.87 & 3.24 \\
\hline Driver 25 & 76 & 104 & 92.46 & 5.42 \\
\hline Driver 26 & 116 & 139 & 128.17 & 5.45 \\
\hline Driver 31 & 72 & 109 & 98.67 & 8.83 \\
\hline Driver 32 & 123 & 134 & 128.19 & 2.12 \\
\hline Total & 58 & 146 & 106.95 & 13.75 \\
\hline
\end{tabular}


Table A - 9: Speed Statistics of Individual Drivers on Rural Freeway under Non-FreeFlow Conditions.

\begin{tabular}{|c|c|c|c|c|}
\hline \multirow{2}{*}{ Driver ID } & \multicolumn{4}{|c|}{ Speed Parameter $(\mathrm{km} / \mathrm{h})$} \\
\cline { 2 - 5 } & Minimum & Maximum & Mean & Std. Deviation \\
\hline Driver 02 & 94 & 114 & 106.68 & 3.10 \\
\hline Driver 03 & 73 & 115 & 106.90 & 5.92 \\
\hline Driver 04 & 69 & 112 & 99.67 & 13.81 \\
\hline Driver 05 & 74 & 110 & 102.43 & 4.26 \\
\hline Driver 06 & 95 & 110 & 103.38 & 2.87 \\
\hline Driver 08 & 72 & 112 & 99.96 & 6.95 \\
\hline Driver 09 & 106 & 119 & 113.16 & 4.35 \\
\hline Driver 10 & 73 & 119 & 104.87 & 5.22 \\
\hline Driver 11 & 74 & 111 & 105.00 & 4.92 \\
\hline Driver 12 & 116 & 131 & 124.57 & 4.94 \\
\hline Driver 13 & 100 & 139 & 124.29 & 8.76 \\
\hline Driver 14 & 96 & 127 & 109.75 & 5.96 \\
\hline Driver 15 & 99 & 116 & 109.33 & 2.85 \\
\hline Driver 16 & 76 & 146 & 115.72 & 13.04 \\
\hline Driver 17 & 86 & 103 & 95.92 & 3.73 \\
\hline Driver 18 & 101 & 133 & 122.66 & 6.08 \\
\hline Driver 19 & 89 & 104 & 97.48 & 3.84 \\
\hline Driver 20 & 100 & 119 & 108.66 & 4.40 \\
\hline Driver 21 & 95 & 115 & 106.95 & 4.51 \\
\hline Driver 22 & 83 & 115 & 108.08 & 3.93 \\
\hline Driver 23 & 89 & 101 & 94.85 & 3.23 \\
\hline Driver 24 & 90 & 105 & 99.37 & 3.68 \\
\hline Driver 25 & 69 & 98 & 88.94 & 5.90 \\
\hline Driver 26 & 75 & 133 & 121.60 & 8.55 \\
\hline Driver 31 & 92 & 117 & 105.94 & 4.31 \\
\hline Driver 32 & 84 & 132 & 123.64 & 8.09 \\
\hline Total & 69 & 146 & 107.38 & 10.22 \\
\hline & & & & \\
\hline & & & \\
\hline
\end{tabular}


Table A - 10: Speed Statistics of Individual Drivers on Urban/Suburban Roads under Mixed Flow Conditions.

\begin{tabular}{|c|c|c|c|c|}
\hline \multirow{2}{*}{ Driver ID } & \multicolumn{4}{|c|}{ Speed Parameter $(\mathrm{km} / \mathrm{h})$} \\
\hline & Minimum & Maximum & Mean & Std. Deviation \\
\hline Driver 02 & 0 & 88 & 58.38 & 17.64 \\
\hline Driver 03 & 0 & 87 & 57.74 & 18.64 \\
\hline Driver 04 & 0 & 87 & 55.91 & 18.81 \\
\hline Driver 05 & 0 & 77 & 54.57 & 15.71 \\
\hline Driver 06 & 0 & 86 & 52.10 & 22.15 \\
\hline Driver 08 & 0 & 90 & 52.10 & 20.11 \\
\hline Driver 09 & 0 & 97 & 57.40 & 18.46 \\
\hline Driver 10 & 0 & 82 & 56.05 & 16.66 \\
\hline Driver 11 & 0 & 77 & 54.88 & 17.53 \\
\hline Driver 12 & 0 & 98 & 56.50 & 22.57 \\
\hline Driver 13 & 0 & 103 & 54.37 & 18.82 \\
\hline Driver 14 & 0 & 83 & 54.40 & 17.90 \\
\hline Driver 15 & 0 & 92 & 58.77 & 19.93 \\
\hline Driver 16 & 0 & 90 & 56.25 & 18.36 \\
\hline Driver 17 & 0 & 78 & 55.88 & 13.40 \\
\hline Driver 18 & 0 & 108 & 61.60 & 23.64 \\
\hline Driver 19 & 0 & 85 & 54.13 & 18.08 \\
\hline Driver 20 & 0 & 86 & 60.73 & 20.93 \\
\hline Driver 21 & 0 & 84 & 53.83 & 17.50 \\
\hline Driver 22 & 0 & 90 & 57.15 & 18.61 \\
\hline Driver 23 & 0 & 81 & 51.41 & 18.12 \\
\hline Driver 24 & 0 & 74 & 54.89 & 16.54 \\
\hline Driver 25 & 0 & 81 & 55.28 & 16.78 \\
\hline Driver 26 & 0 & 83 & 57.18 & 16.48 \\
\hline Driver 31 & 0 & 99 & 60.47 & 19.60 \\
\hline Driver 32 & 0 & 97 & 62.00 & 19.63 \\
\hline Total & 0 & 108 & 56.18 & 18.85 \\
\hline
\end{tabular}


Table A - 11: Speed Statistics of Individual Drivers on Urban/Suburban Roads under

Free-Flow Conditions.

\begin{tabular}{|c|c|c|c|c|}
\hline \multirow{2}{*}{ Driver ID } & \multicolumn{4}{|c|}{ Speed Parameter $(\mathrm{km} / \mathrm{h})$} \\
\cline { 2 - 5 } & Minimum & Maximum & Mean & Std. Deviation \\
\hline Driver 02 & 0 & 88 & 66.38 & 20.59 \\
\hline Driver 03 & 0 & 80 & 59.87 & 16.90 \\
\hline Driver 04 & 0 & 77 & 55.36 & 18.41 \\
\hline Driver 05 & 0 & 76 & 54.54 & 14.95 \\
\hline Driver 06 & 0 & 86 & 63.44 & 19.63 \\
\hline Driver 08 & 0 & 90 & 57.58 & 21.21 \\
\hline Driver 09 & 0 & 97 & 66.60 & 21.78 \\
\hline Driver 10 & 0 & 82 & 58.12 & 15.87 \\
\hline Driver 11 & 0 & 77 & 58.23 & 17.15 \\
\hline Driver 12 & 0 & 92 & 61.88 & 17.22 \\
\hline Driver 13 & 0 & 99 & 63.12 & 21.02 \\
\hline Driver 14 & 0 & 83 & 58.23 & 18.79 \\
\hline Driver 15 & 0 & 87 & 56.65 & 21.75 \\
\hline Driver 16 & 0 & 90 & 61.02 & 18.09 \\
\hline Driver 17 & 0 & 78 & 54.48 & 11.61 \\
\hline Driver 18 & 0 & 103 & 74.86 & 23.13 \\
\hline Driver 19 & 0 & 85 & 64.37 & 18.74 \\
\hline Driver 20 & 0 & 85 & 63.01 & 20.90 \\
\hline Driver 21 & 0 & 77 & 56.53 & 12.40 \\
\hline Driver 22 & 0 & 90 & 63.92 & 16.07 \\
\hline Driver 23 & 0 & 79 & 53.35 & 20.42 \\
\hline Driver 24 & 0 & 74 & 60.60 & 11.12 \\
\hline Driver 25 & 0 & 81 & 58.88 & 14.74 \\
\hline Driver 26 & 0 & 74 & 61.59 & 12.97 \\
\hline Driver 31 & 0 & 99 & 61.39 & 19.33 \\
\hline Driver 32 & 0 & 97 & 70.40 & 21.75 \\
\hline Total & 0 & 103 & 60.45 & 18.16 \\
\hline & & & & \\
\hline & 03 & \\
\hline
\end{tabular}


Table A - 12: Speed Statistics of Individual Drivers on Urban/Suburban Roads under Non-Free-Flow Conditions.

\begin{tabular}{|c|c|c|c|c|}
\hline \multirow{2}{*}{ Driver ID } & \multicolumn{4}{|c|}{ Speed Parameter $(\mathrm{km} / \mathrm{h})$} \\
\cline { 2 - 5 } & Minimum & Maximum & Mean & Std. Deviation \\
\hline Driver 02 & 0 & 87 & 53.99 & 13.99 \\
\hline Driver 03 & 0 & 87 & 55.93 & 19.84 \\
\hline Driver 04 & 0 & 87 & 56.04 & 18.91 \\
\hline Driver 05 & 0 & 77 & 54.60 & 16.53 \\
\hline Driver 06 & 0 & 86 & 47.97 & 21.57 \\
\hline Driver 08 & 0 & 83 & 49.09 & 18.84 \\
\hline Driver 09 & 0 & 83 & 55.01 & 16.70 \\
\hline Driver 10 & 0 & 75 & 50.48 & 17.48 \\
\hline Driver 11 & 0 & 75 & 50.30 & 17.04 \\
\hline Driver 12 & 0 & 98 & 54.00 & 24.27 \\
\hline Driver 13 & 0 & 103 & 52.91 & 18.04 \\
\hline Driver 14 & 0 & 82 & 52.71 & 17.23 \\
\hline Driver 15 & 0 & 92 & 59.77 & 18.95 \\
\hline Driver 16 & 0 & 86 & 50.92 & 17.16 \\
\hline Driver 17 & 0 & 77 & 57.20 & 14.78 \\
\hline Driver 18 & 0 & 108 & 55.89 & 21.48 \\
\hline Driver 19 & 0 & 75 & 49.92 & 16.02 \\
\hline Driver 20 & 0 & 86 & 58.65 & 20.77 \\
\hline Driver 21 & 0 & 84 & 53.04 & 18.67 \\
\hline Driver 22 & 0 & 78 & 52.01 & 18.78 \\
\hline Driver 23 & 0 & 81 & 51.01 & 17.60 \\
\hline Driver 24 & 0 & 71 & 46.22 & 19.42 \\
\hline Driver 25 & 0 & 75 & 44.47 & 17.89 \\
\hline Driver 26 & 0 & 83 & 56.25 & 17.00 \\
\hline Driver 31 & 0 & 83 & 58.57 & 20.06 \\
\hline Driver 32 & 0 & 83 & 58.10 & 17.24 \\
\hline Total & 0 & 108 & 53.44 & 18.77 \\
\hline & & & & \\
\hline & 08 & \\
\hline
\end{tabular}




\section{APPENDIX B: SPEED DISTRIBUTION OF INDIVIDUAL DRIVERS AND THE ESTIMATED SPEED DISTRIBUTION OF THE COMMON DRIVER POPULATION}




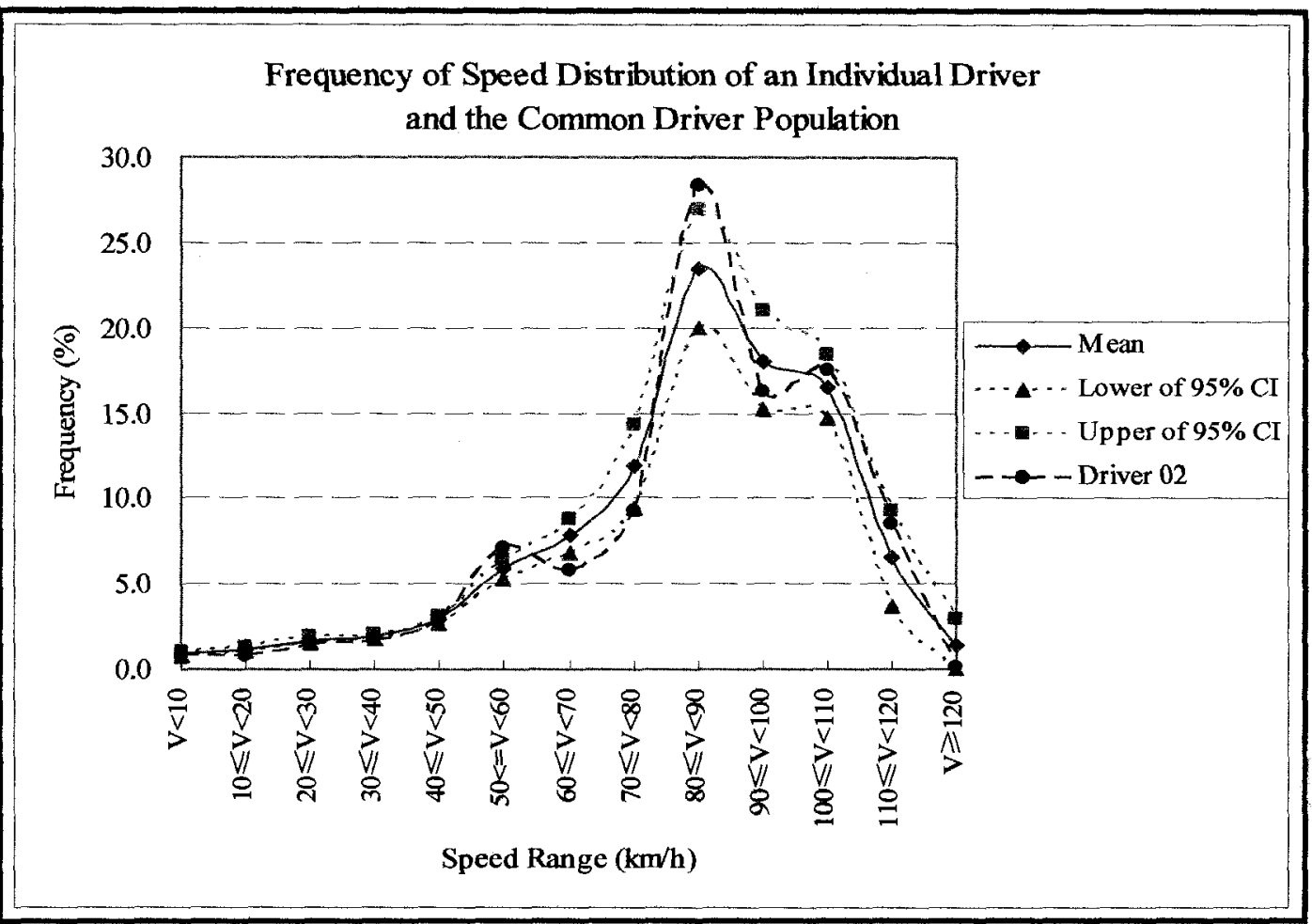

Figure B - 1: Speed Distribution of Driver 02 and the Common Driver Population.

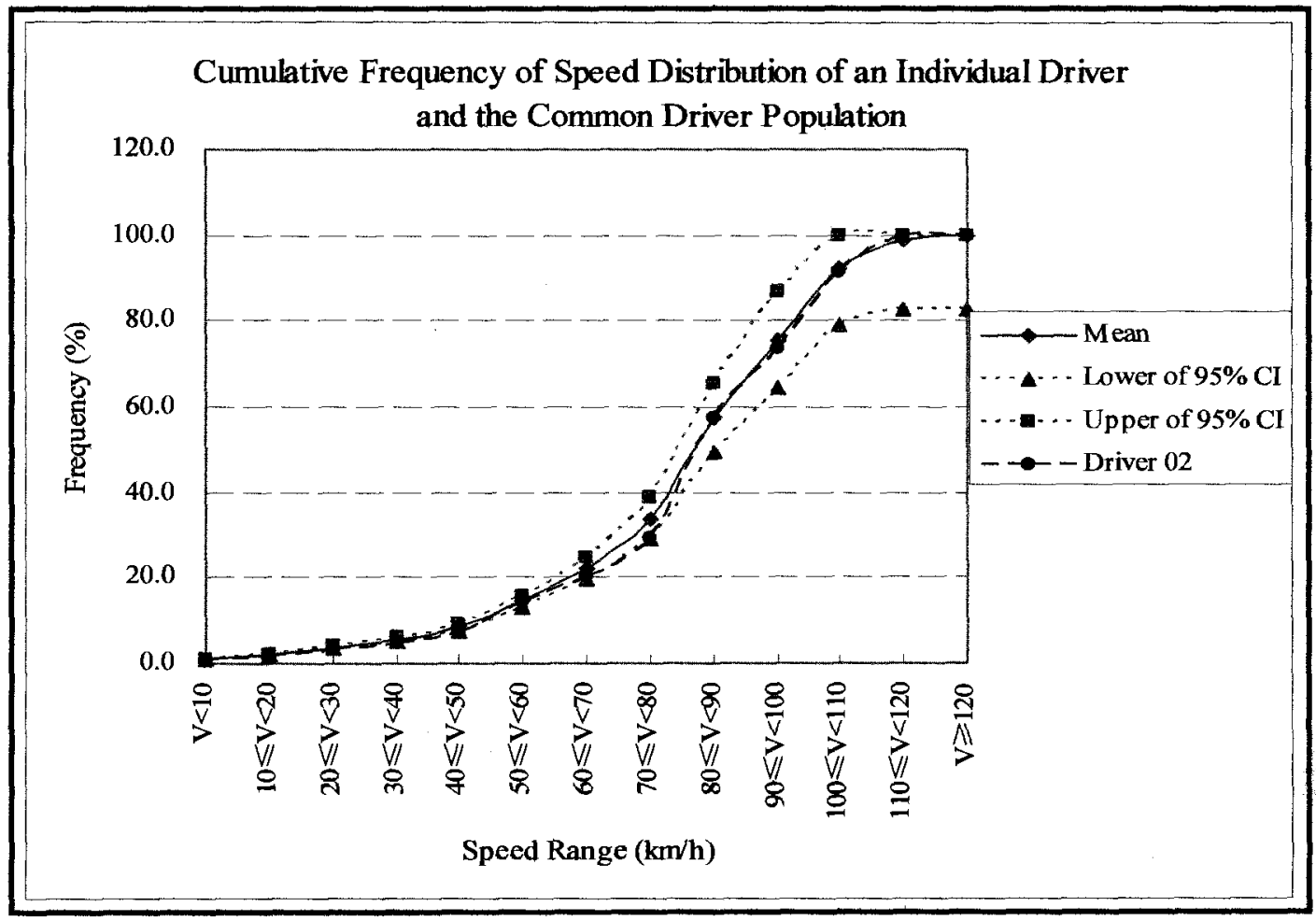

Figure B - 2: Cumulative Frequency of Speed Distribution of Driver 02 and the Common Driver Population. 


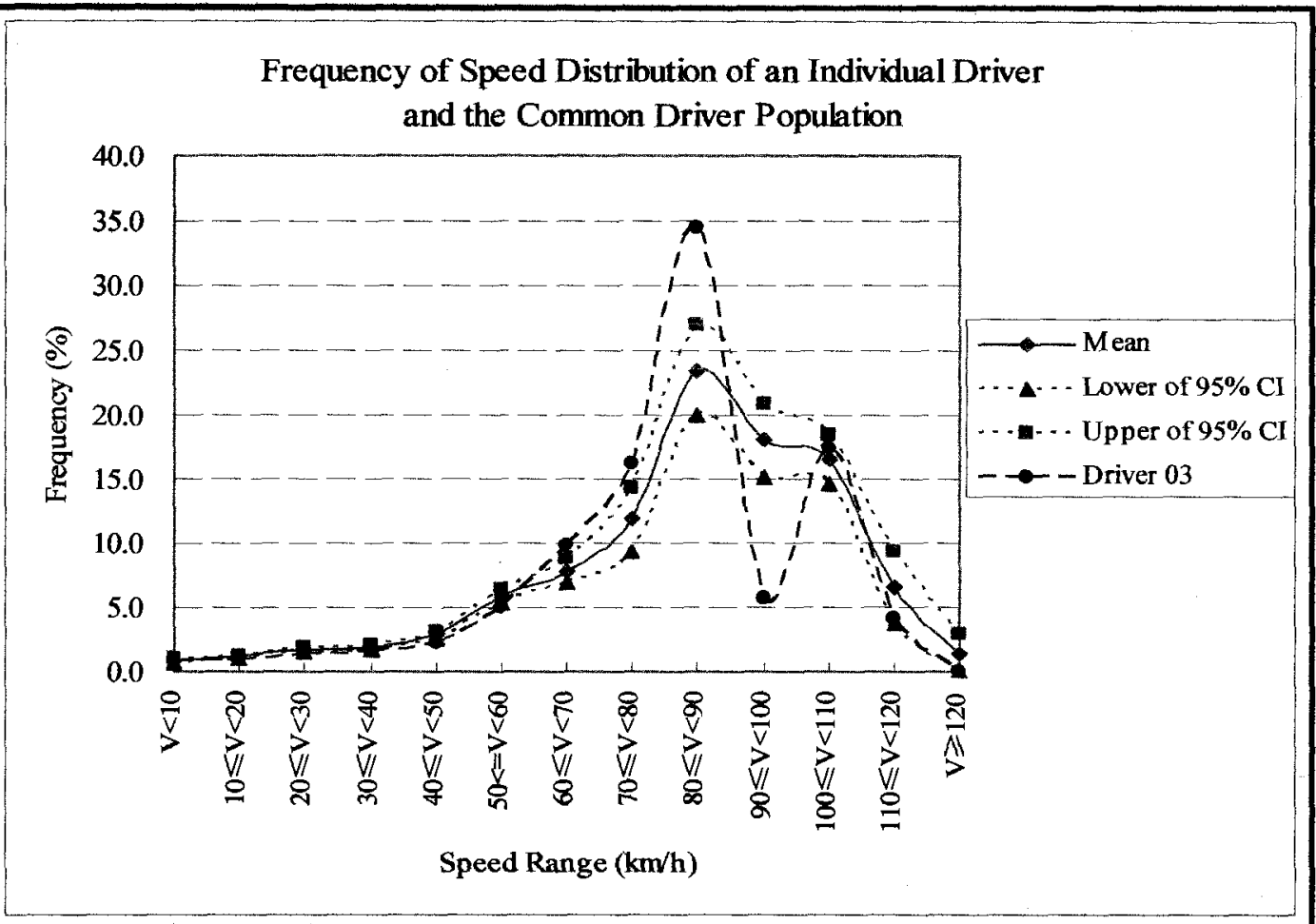

Figure B - 3: Speed Distribution of Driver 03 and the Common Driver Population.

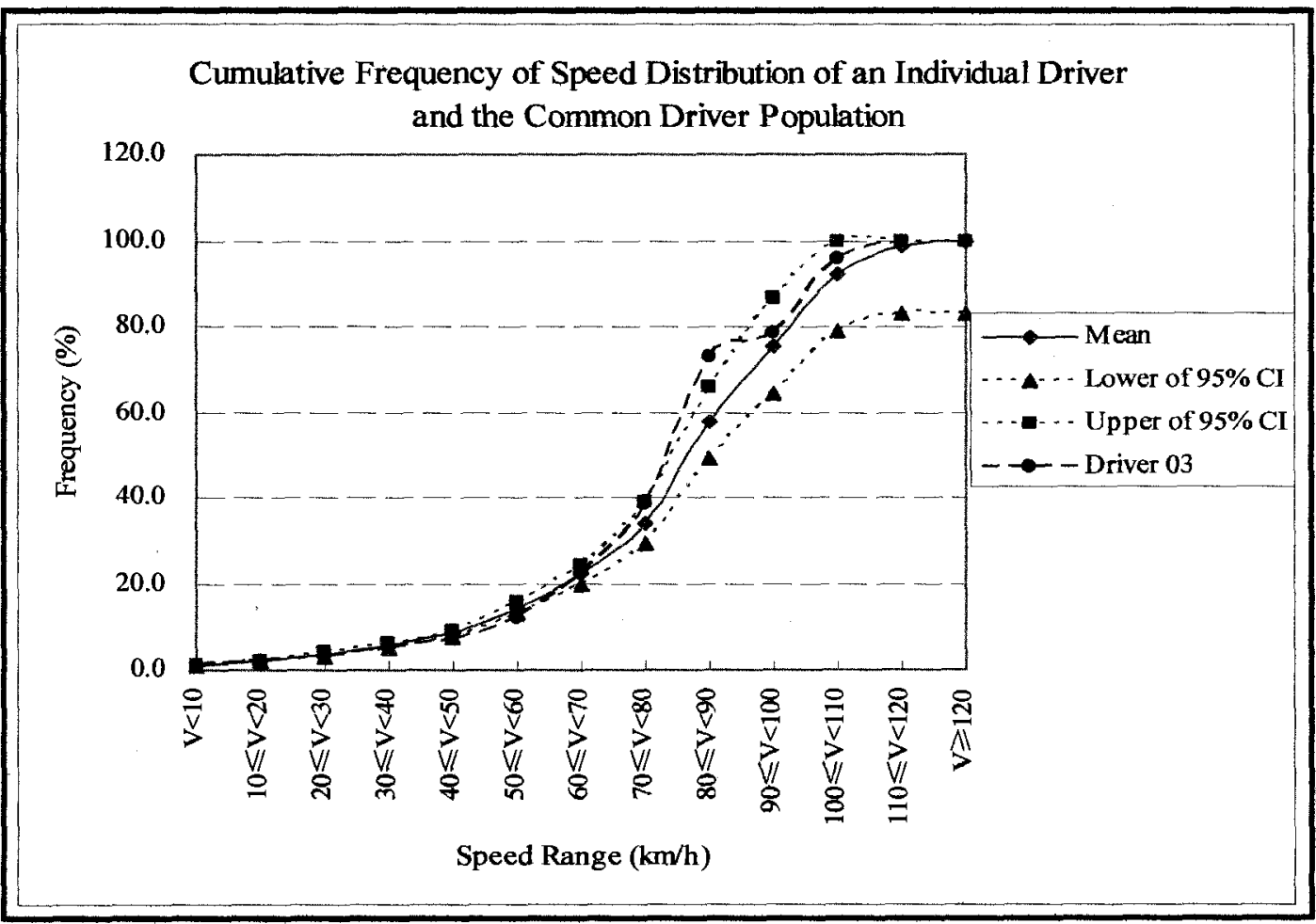

Figure B - 4: Cumulative Frequency of Speed Distribution of Driver 03 and the Common Driver Population. 


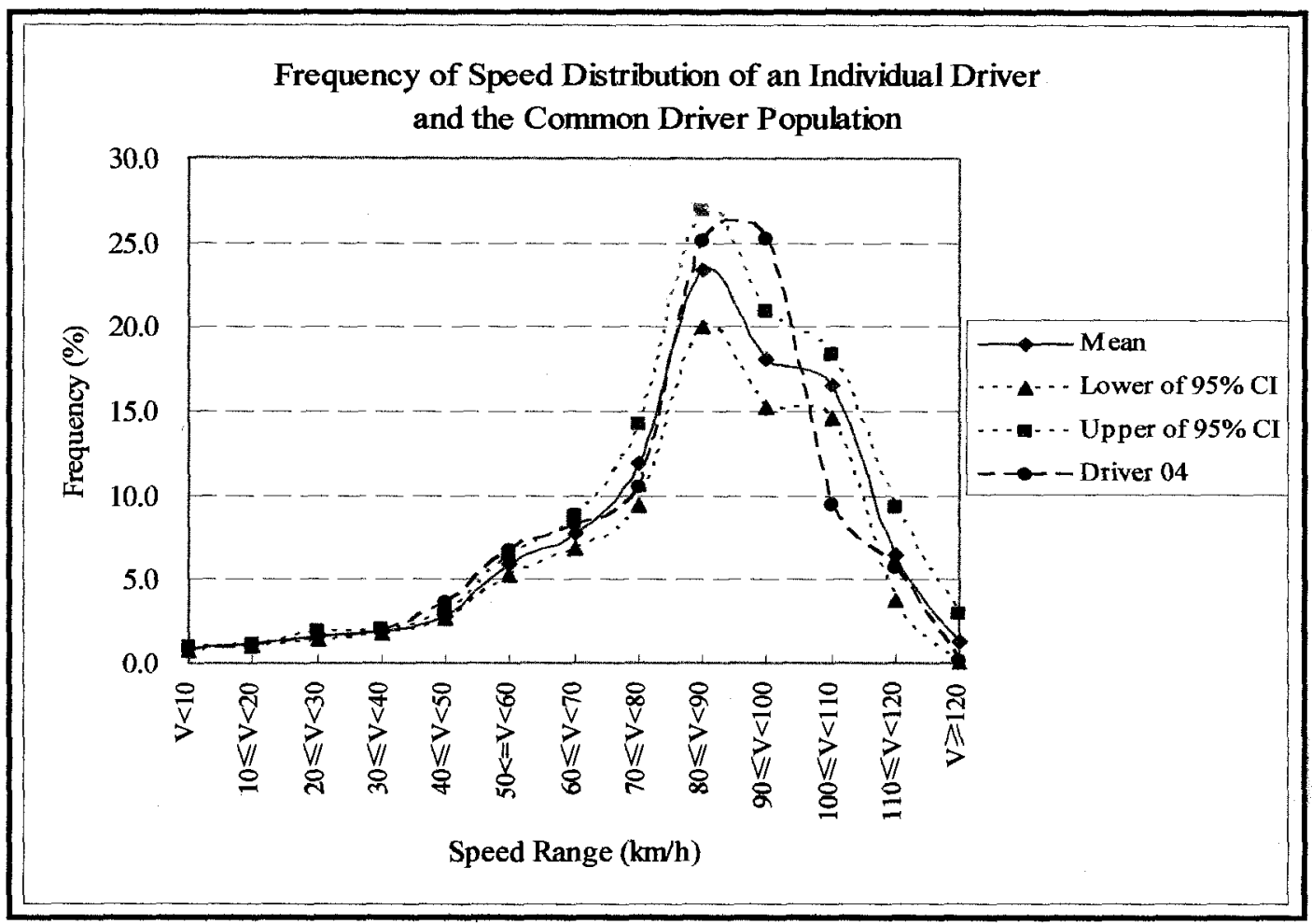

Figure B - 5: Speed Distribution of Driver 04 and the Common Driver Population.

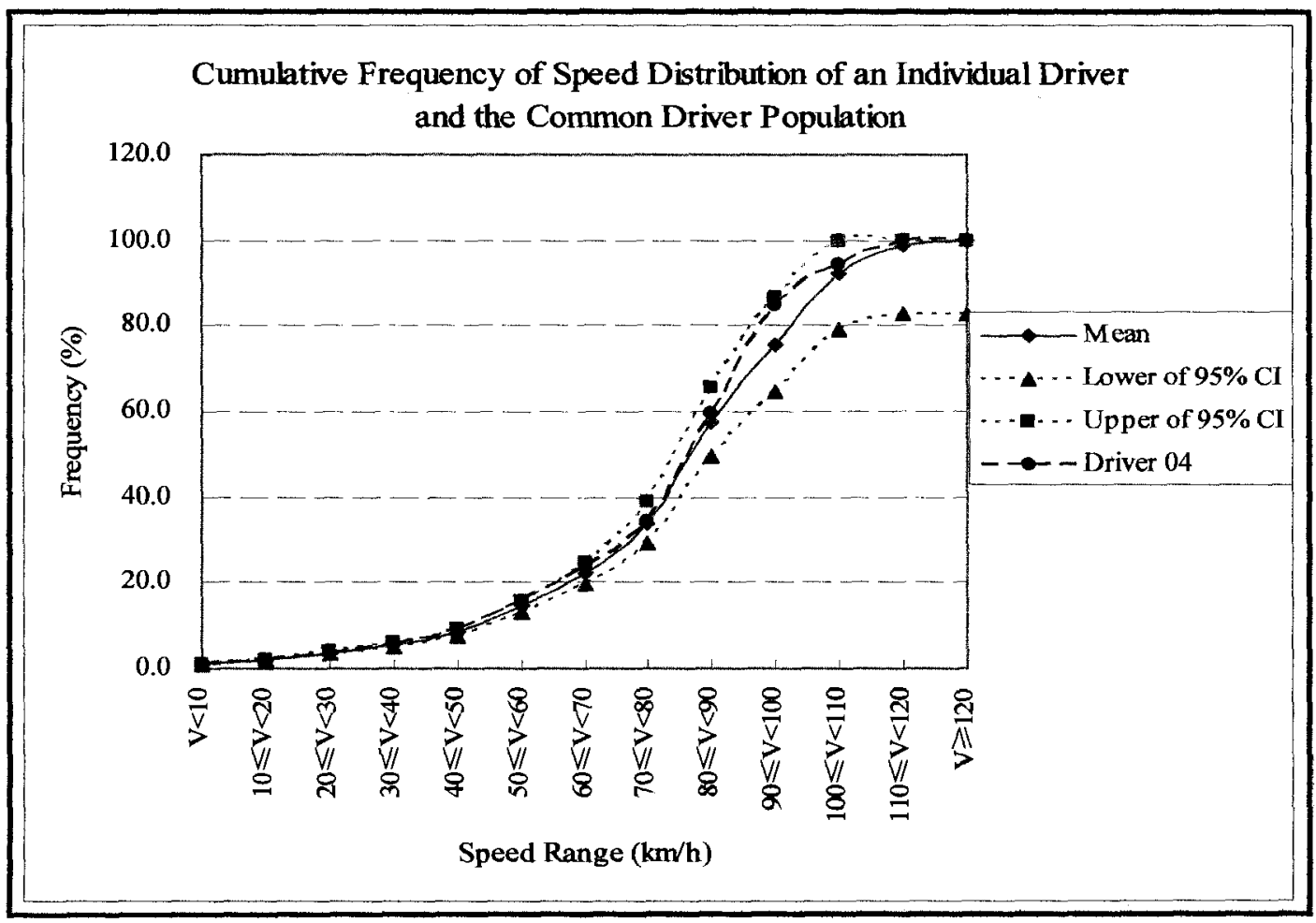

Figure B - 6: Cumulative Frequency of Speed Distribution of Driver 04 and the Common Driver Population. 


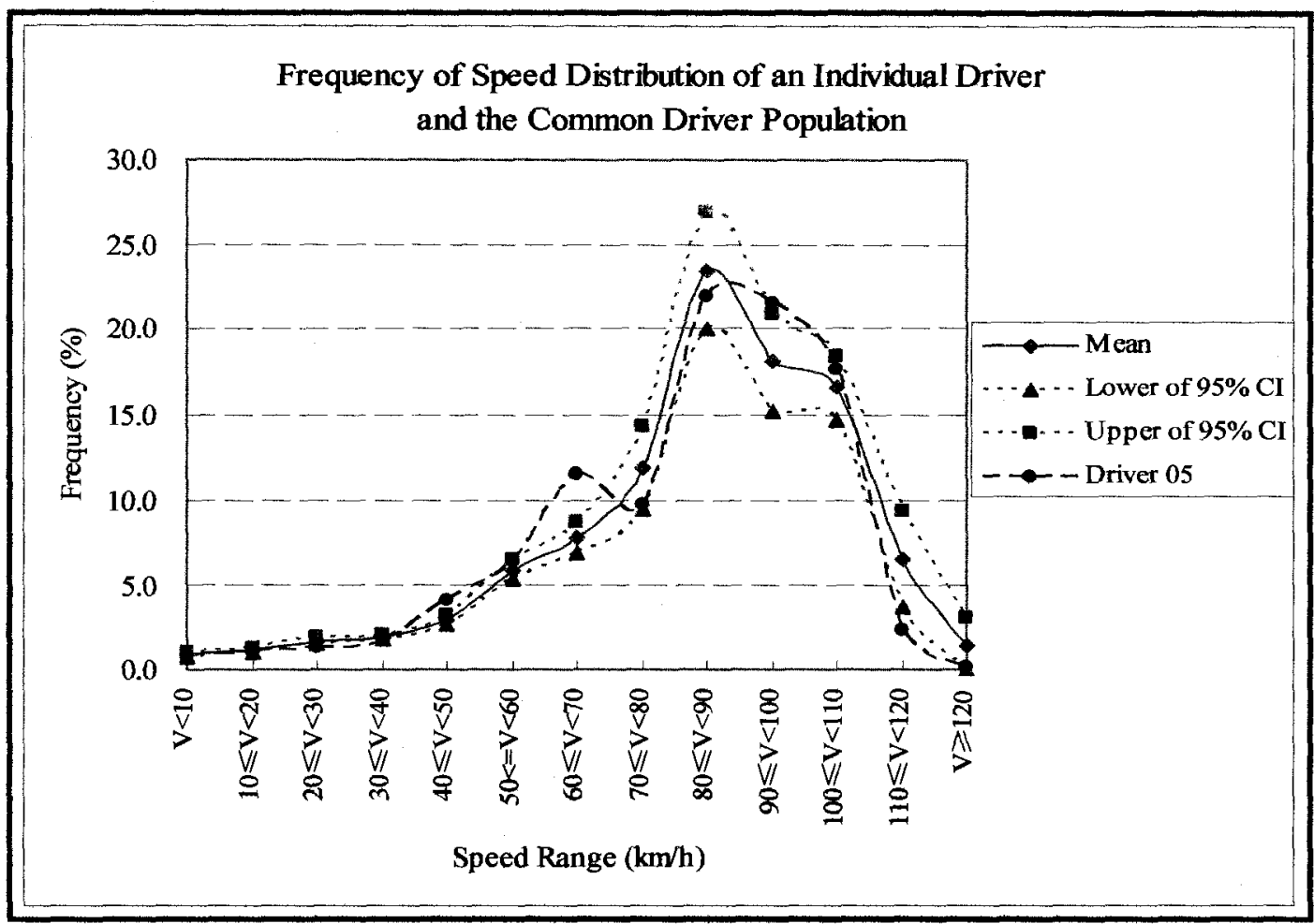

Figure B - 7: Speed Distribution of Driver 05 and the Common Driver Population.

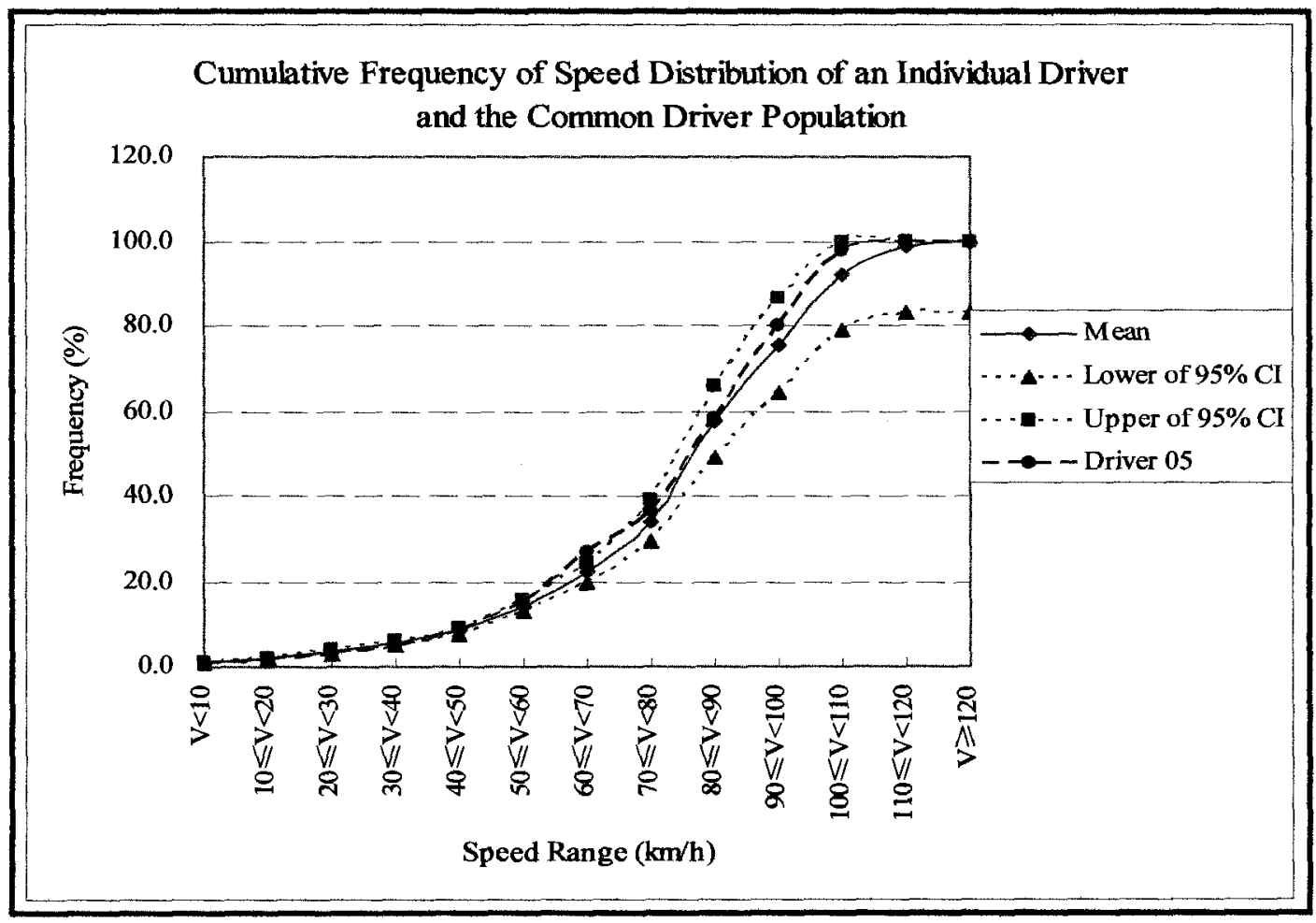

Figure B - 8: Cumulative Frequency of Speed Distribution of Driver 05 and the Common Driver Population. 


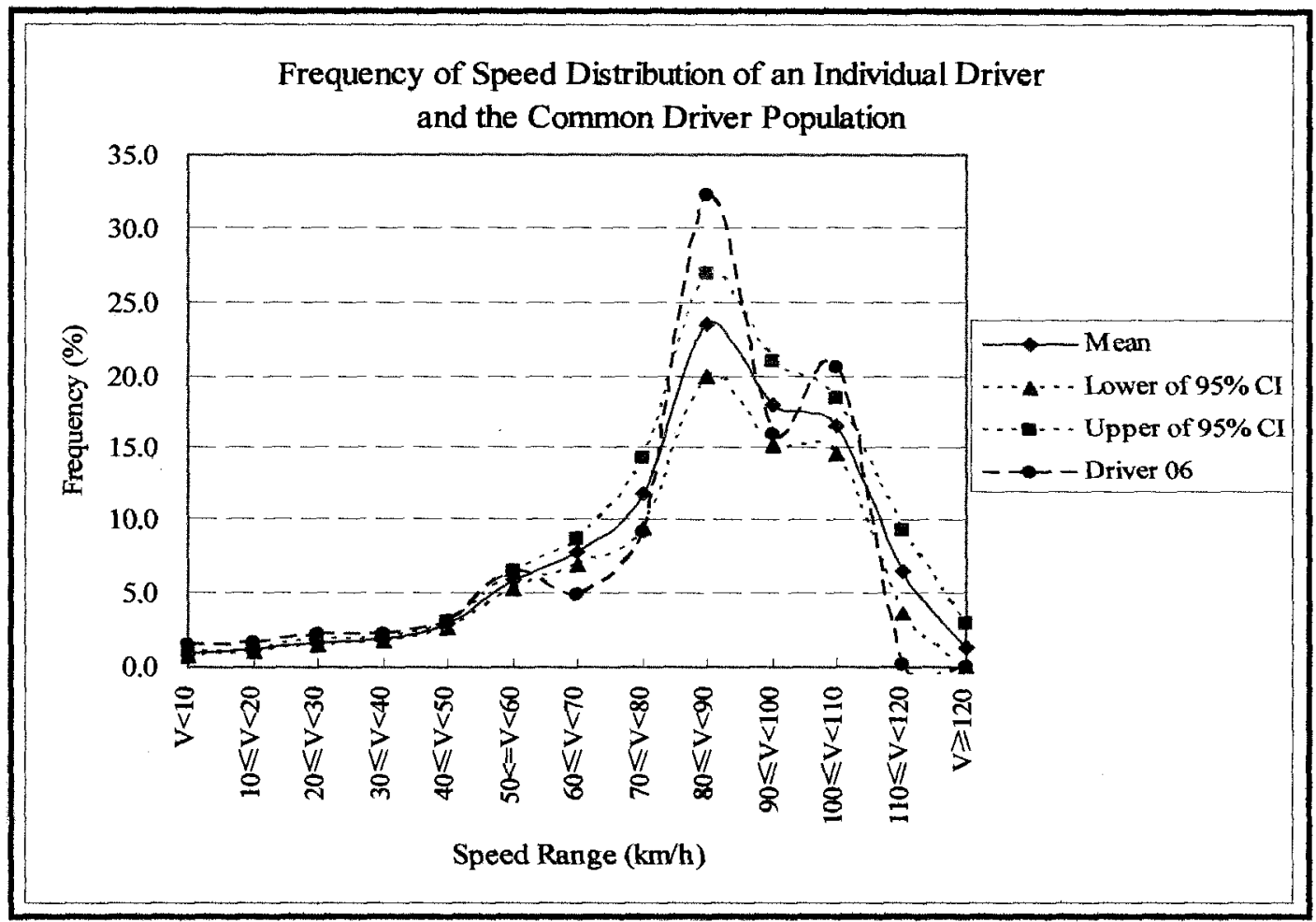

Figure B - 9: Speed Distribution of Driver 06 and the Common Driver Population.

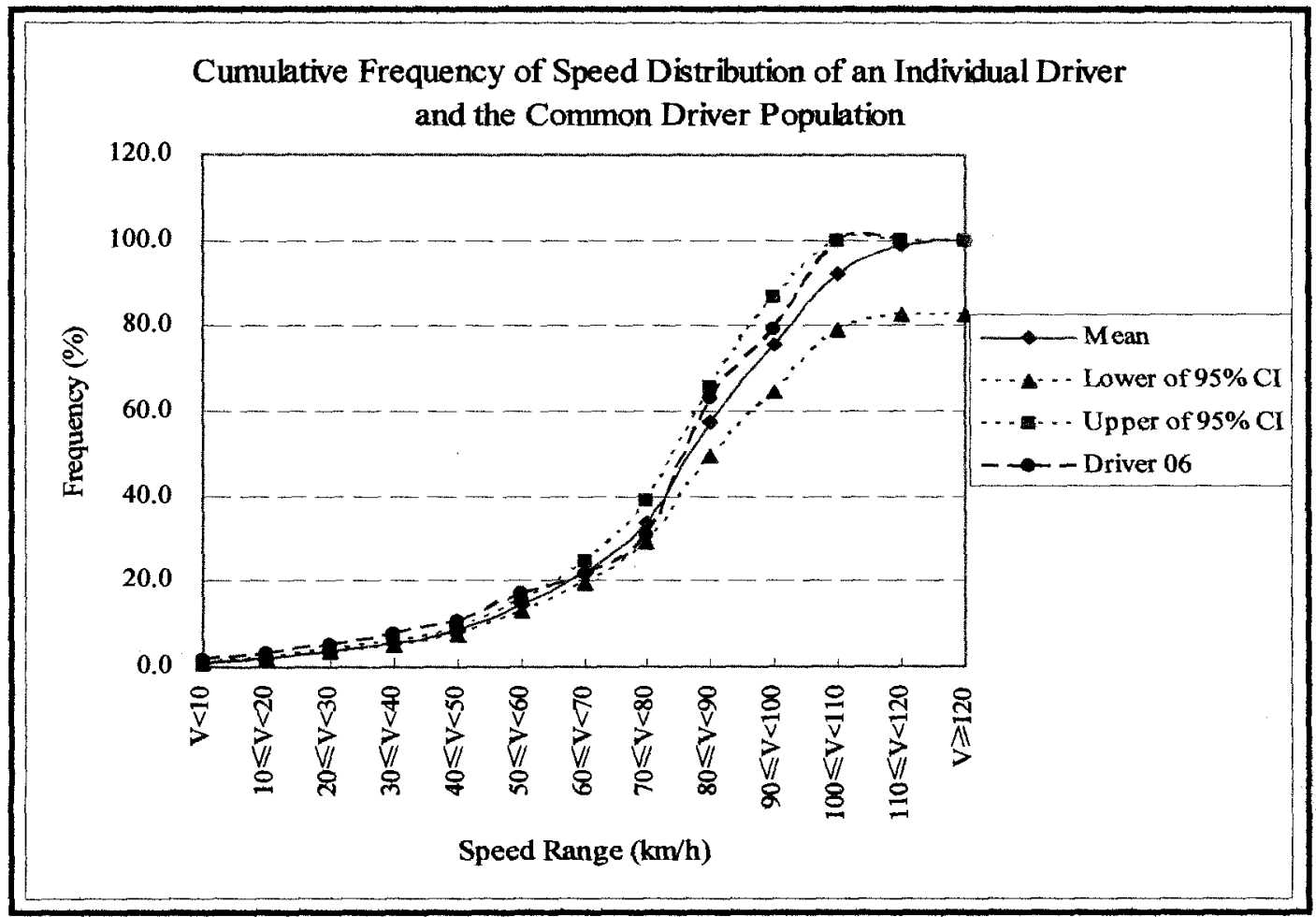

Figure B - 10: Cumulative Frequency of Speed Distribution of Driver 06 and the Common Driver Population. 


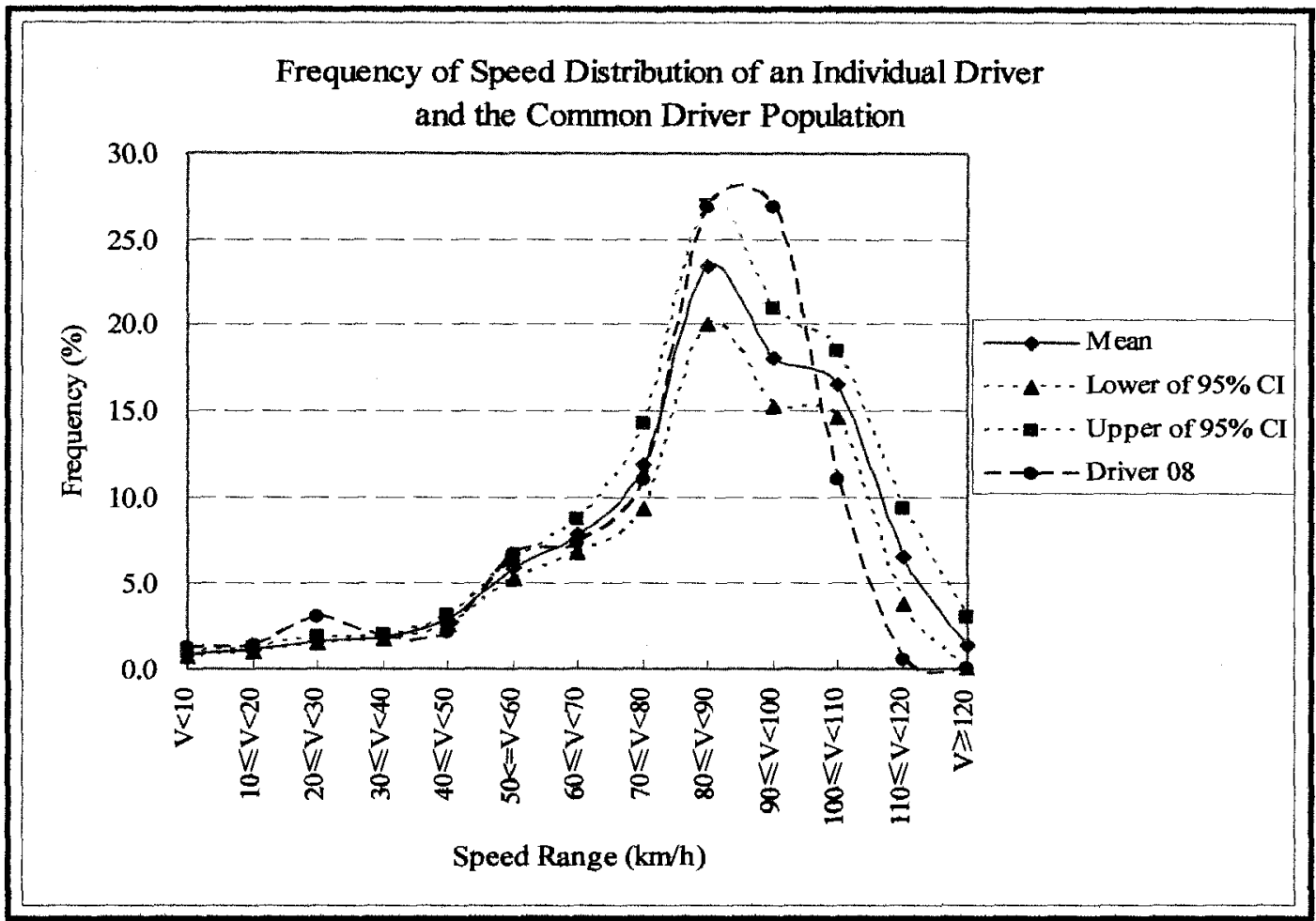

Figure B - 11: Speed Distribution of Driver 08 and the Common Driver Population.

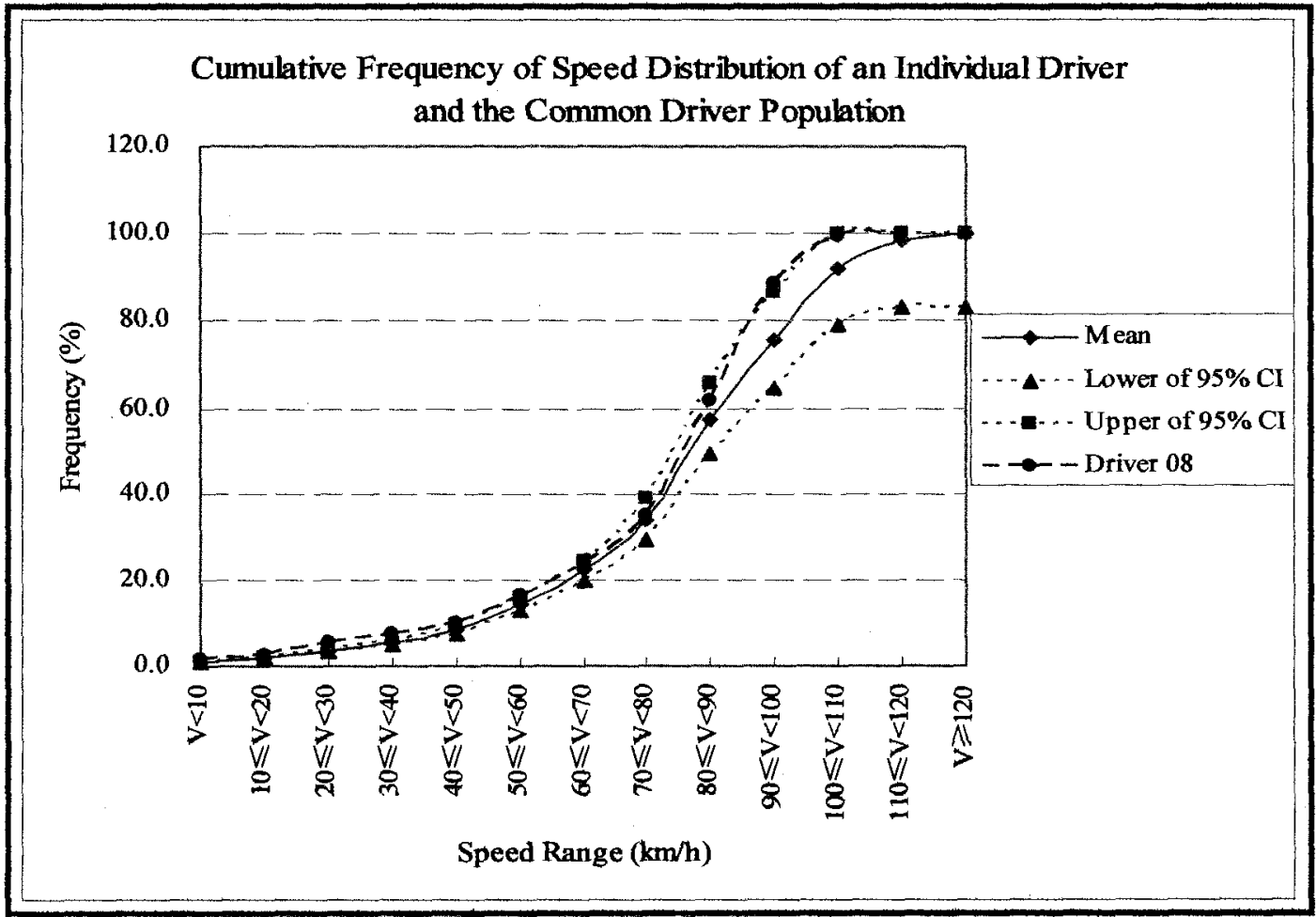

Figure B - 12: Cumulative Frequency of Speed Distribution of Driver 08 and the Common Driver Population. 


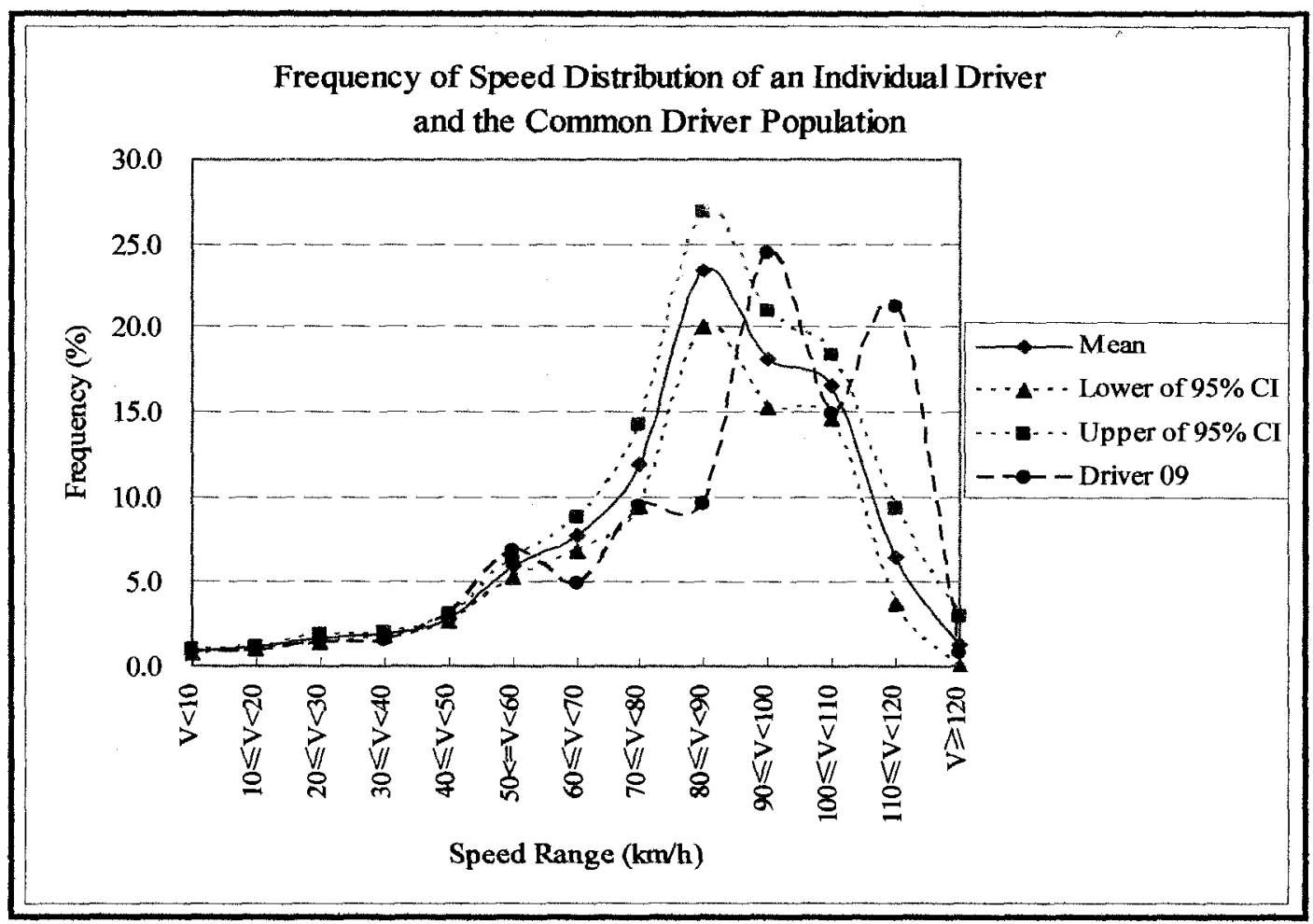

Figure B - 13: Speed Distribution of Driver 09 and the Common Driver Population.

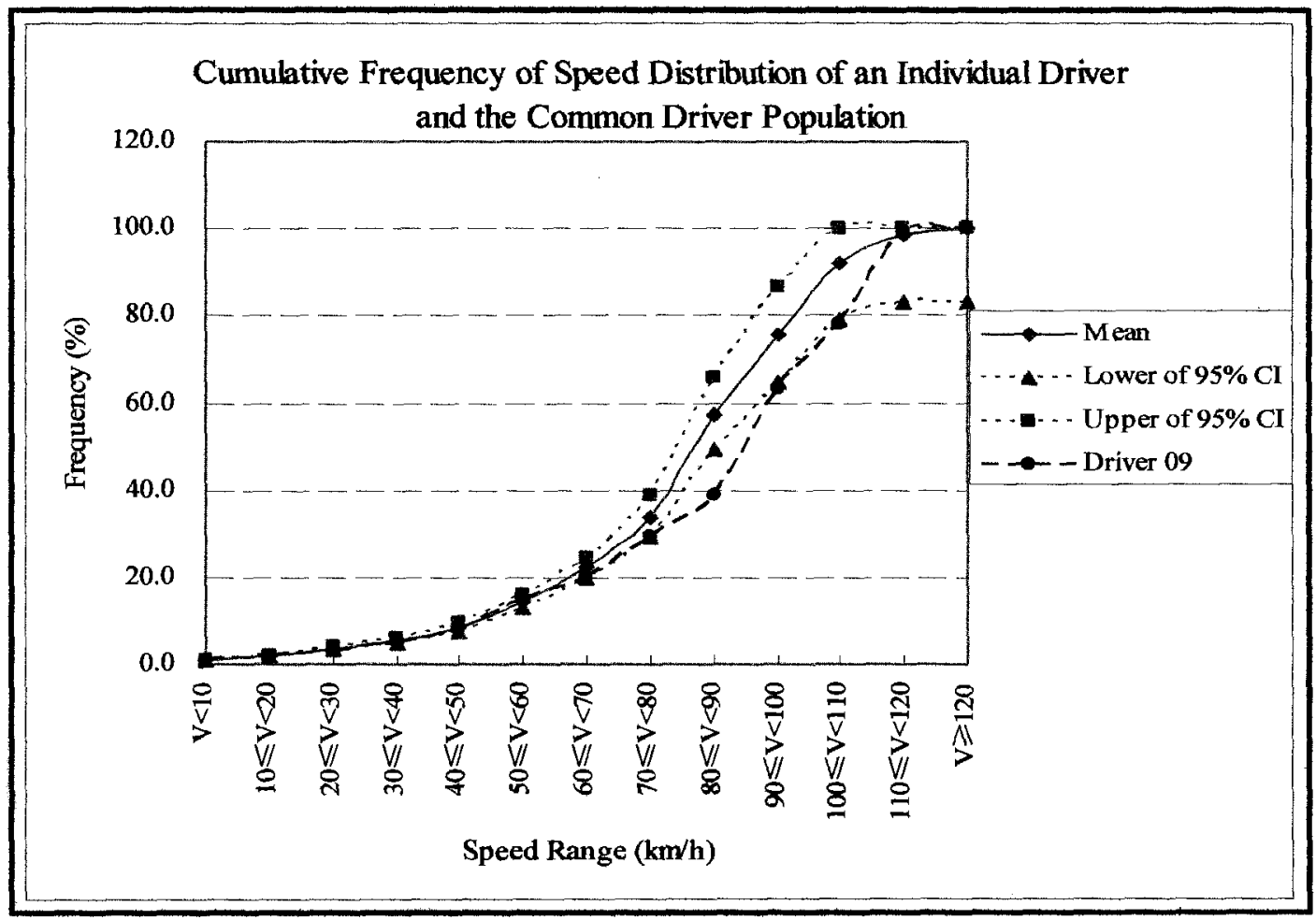

Figure B - 14: Cumulative Frequency of Speed Distribution of Driver 09 and the Common Driver Population. 


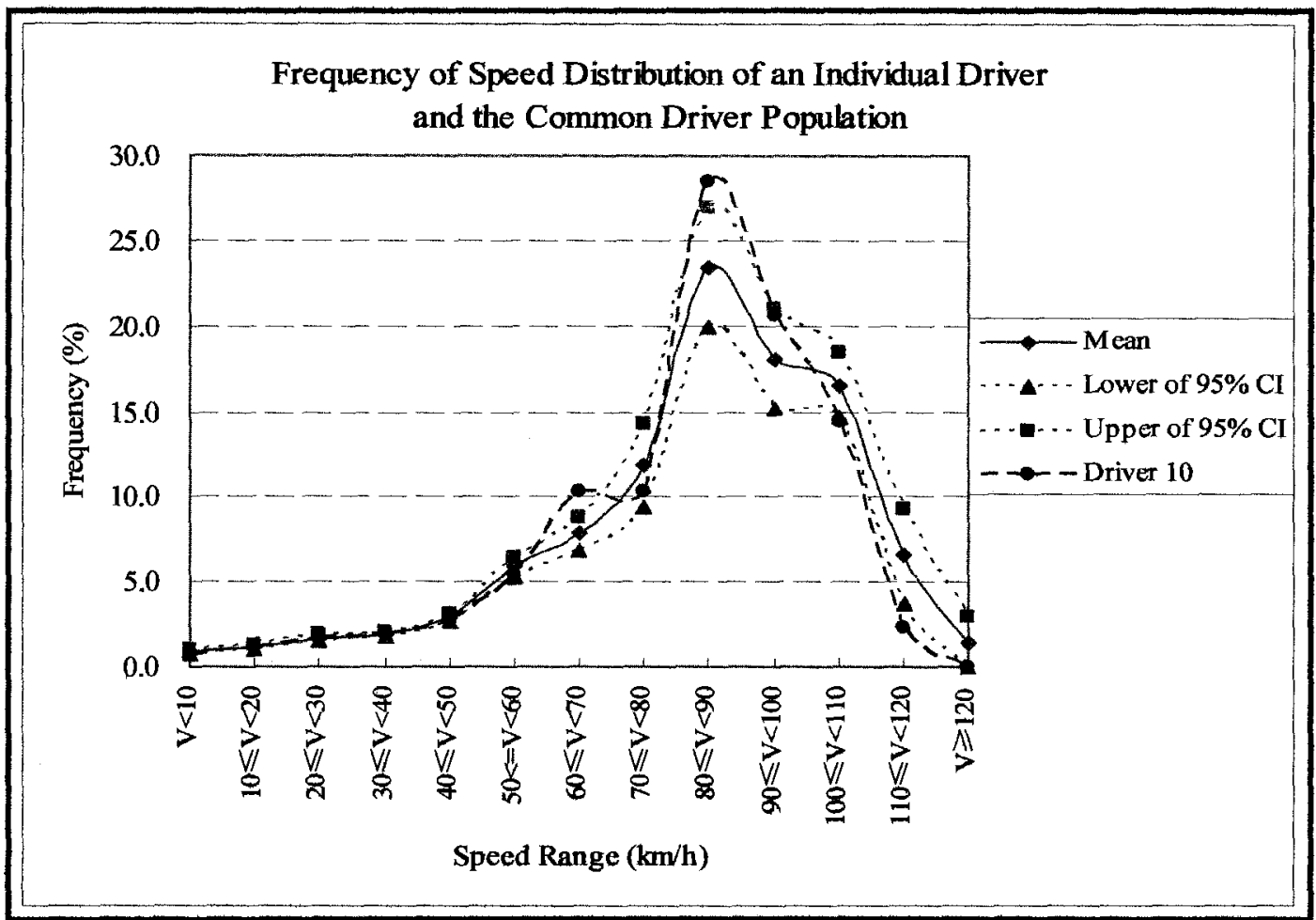

Figure B - 15: Speed Distribution of Driver 10 and the Common Driver Population.

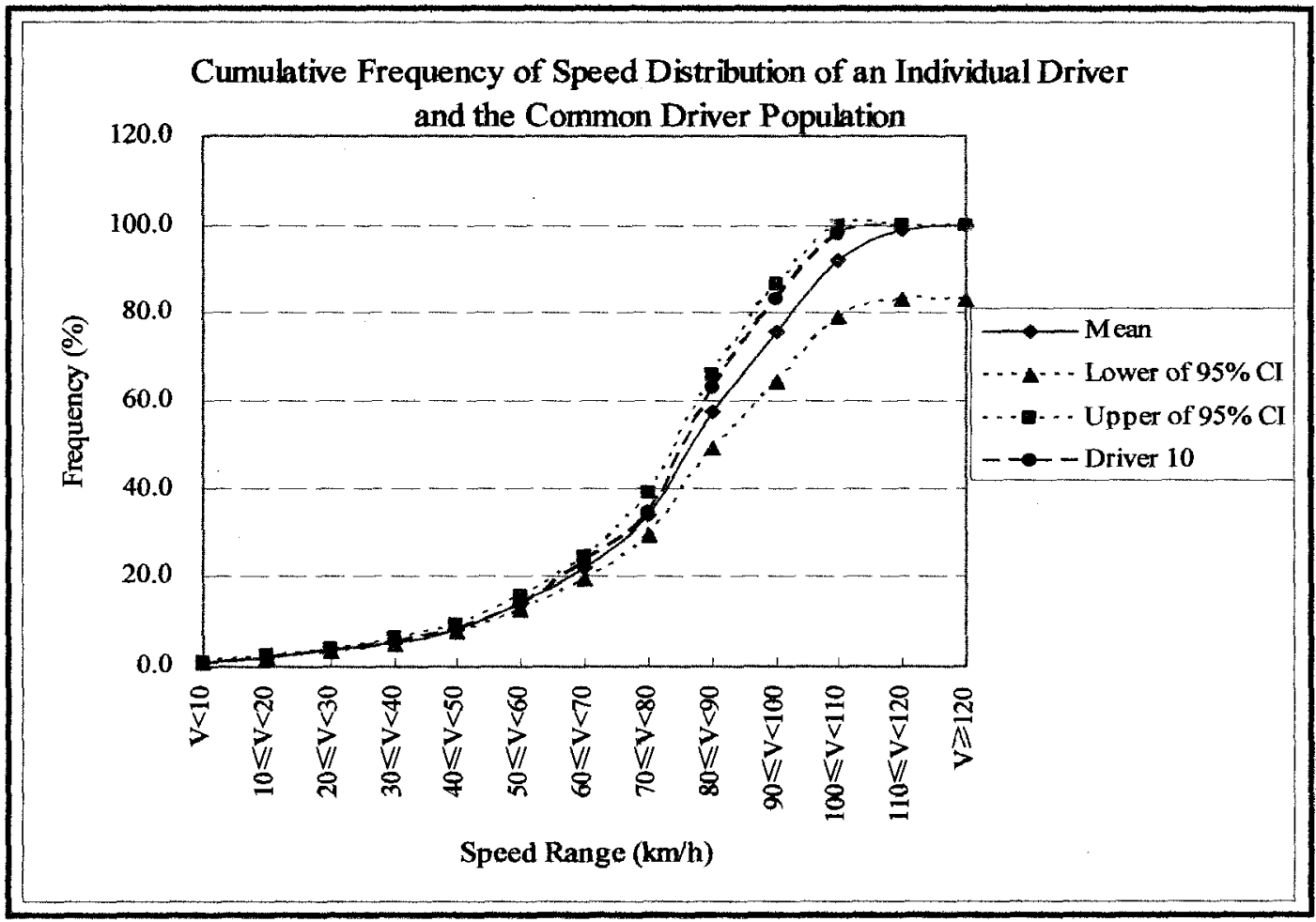

Figure B - 16: Cumulative Frequency of Speed Distribution of Driver 10 and the Common Driver Population. 


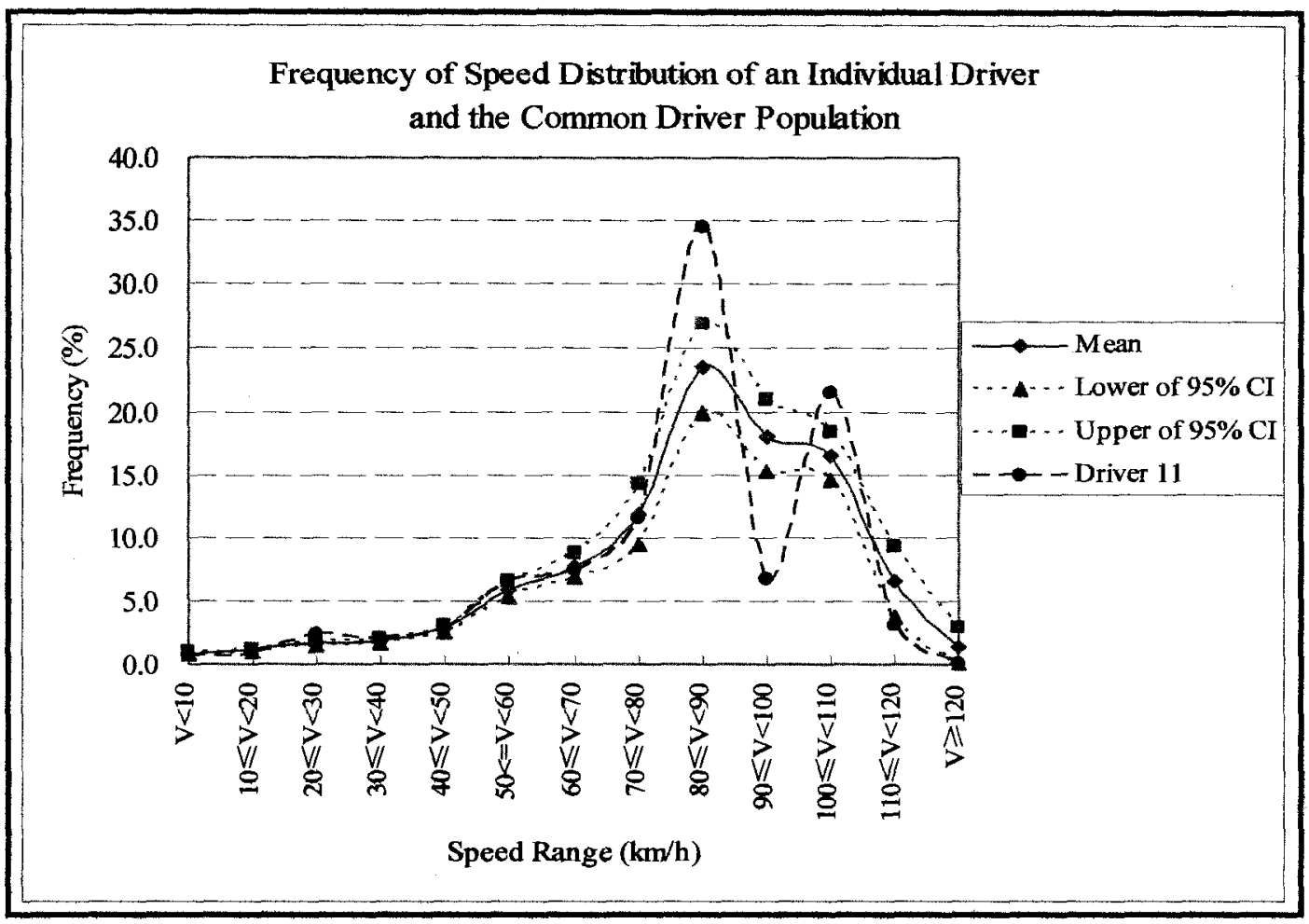

Figure B - 17: Speed Distribution of Driver 11 and the Common Driver Population.

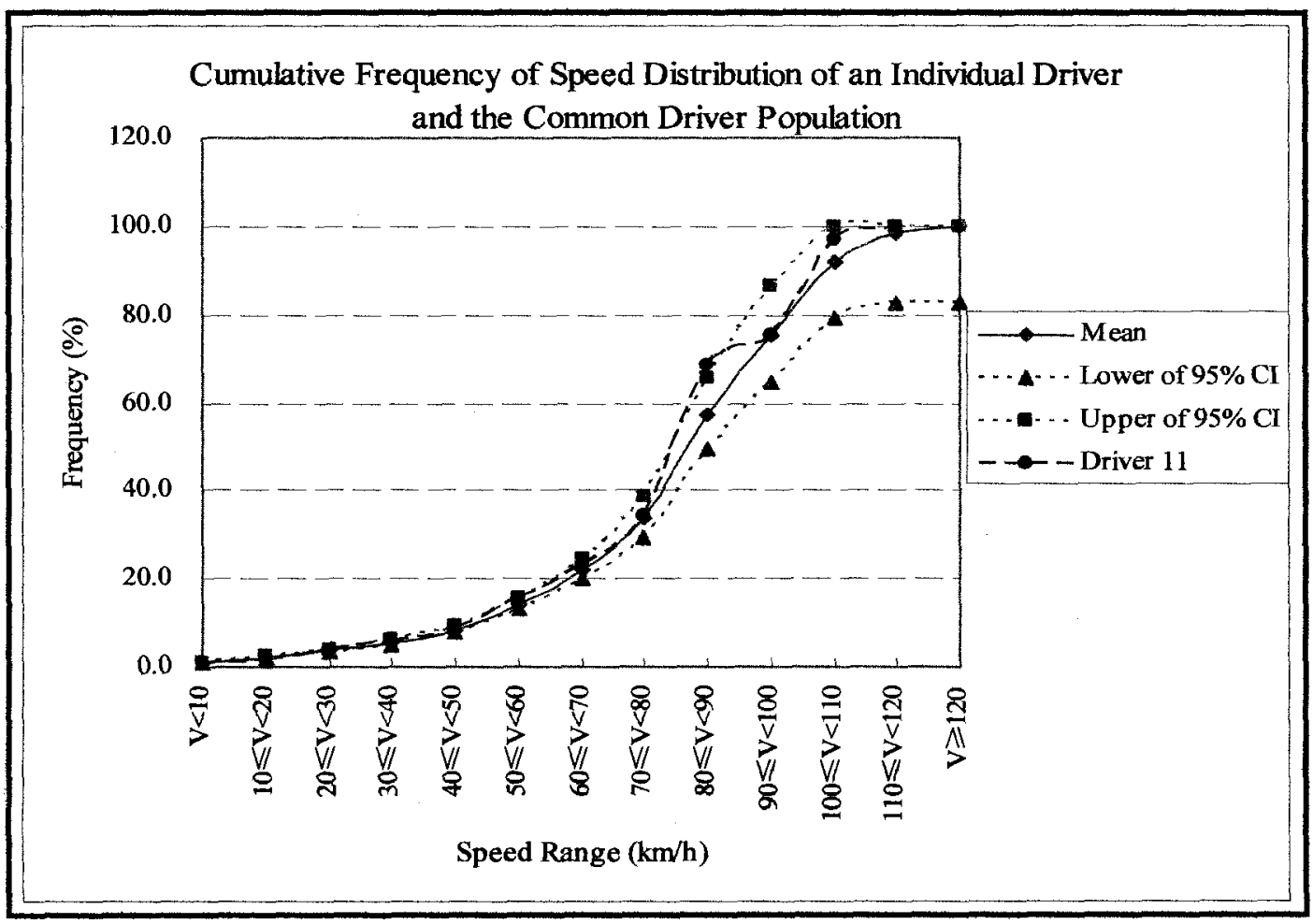

Figure B - 18: Cumulative Frequency of Speed Distribution of Driver 11 and the Common Driver Population. 


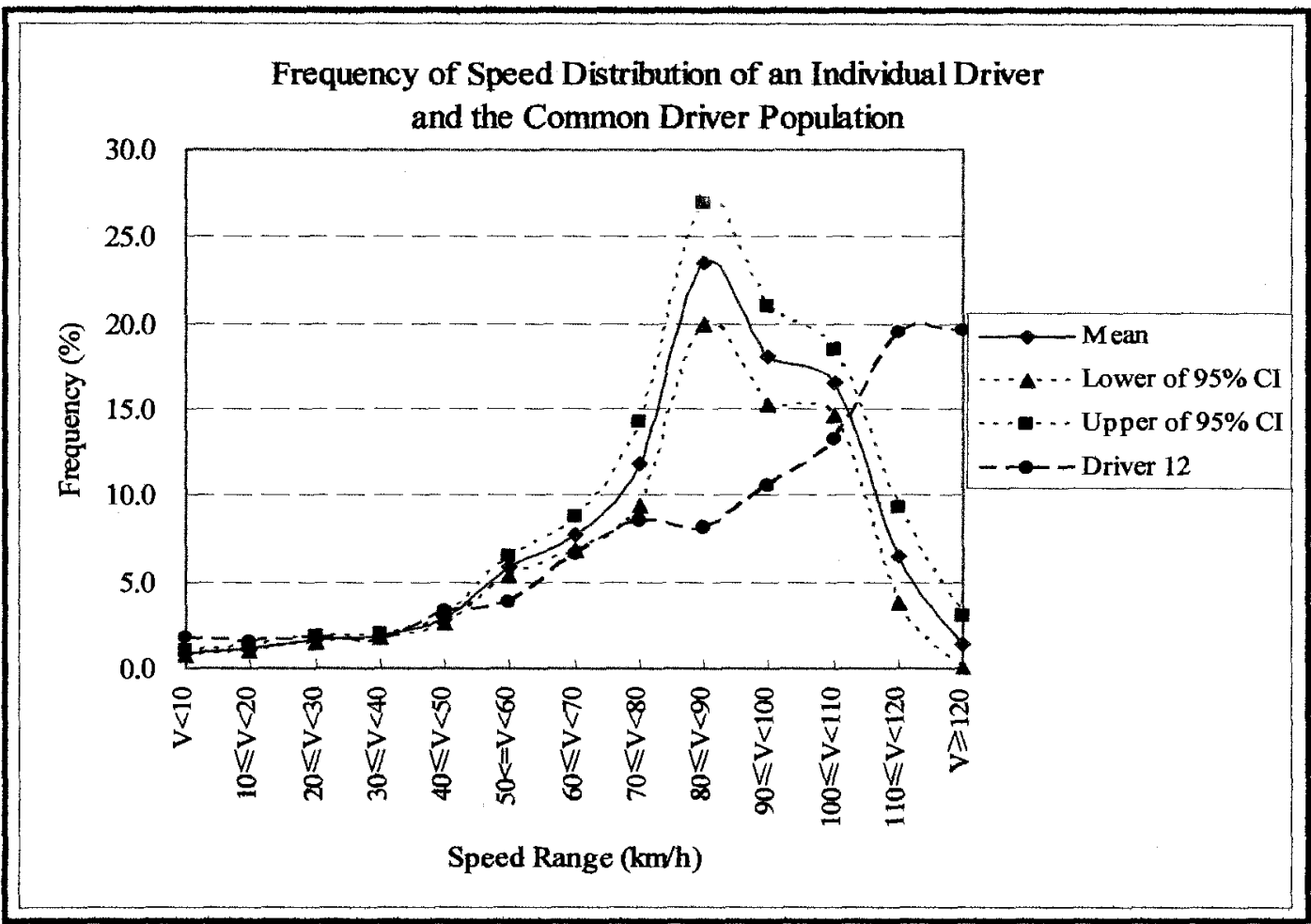

Figure B - 19: Speed Distribution of Driver 12 and the Common Driver Population.

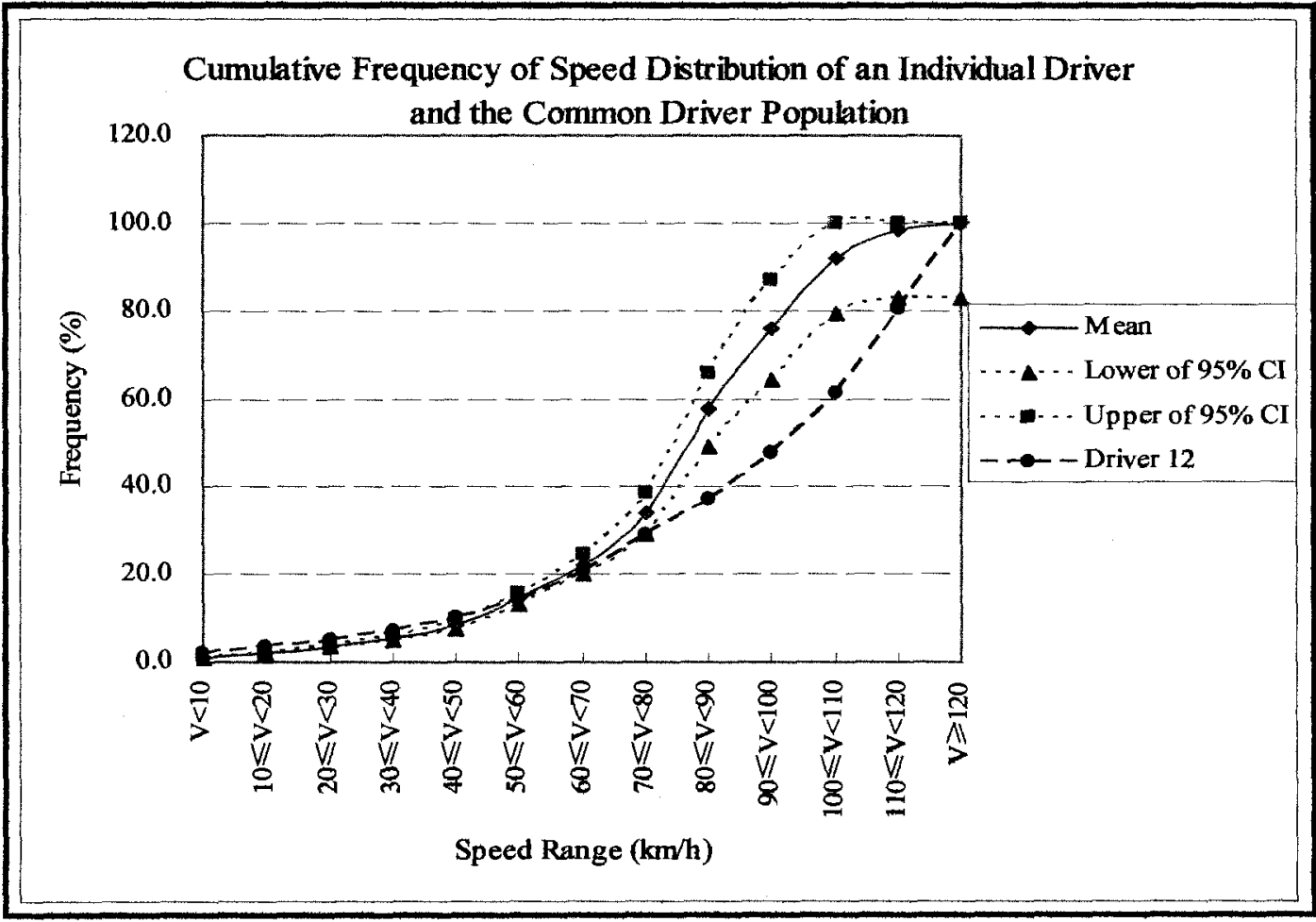

Figure B - 20: Cumulative Frequency of Speed Distribution of Driver 12 and the Common Driver Population. 


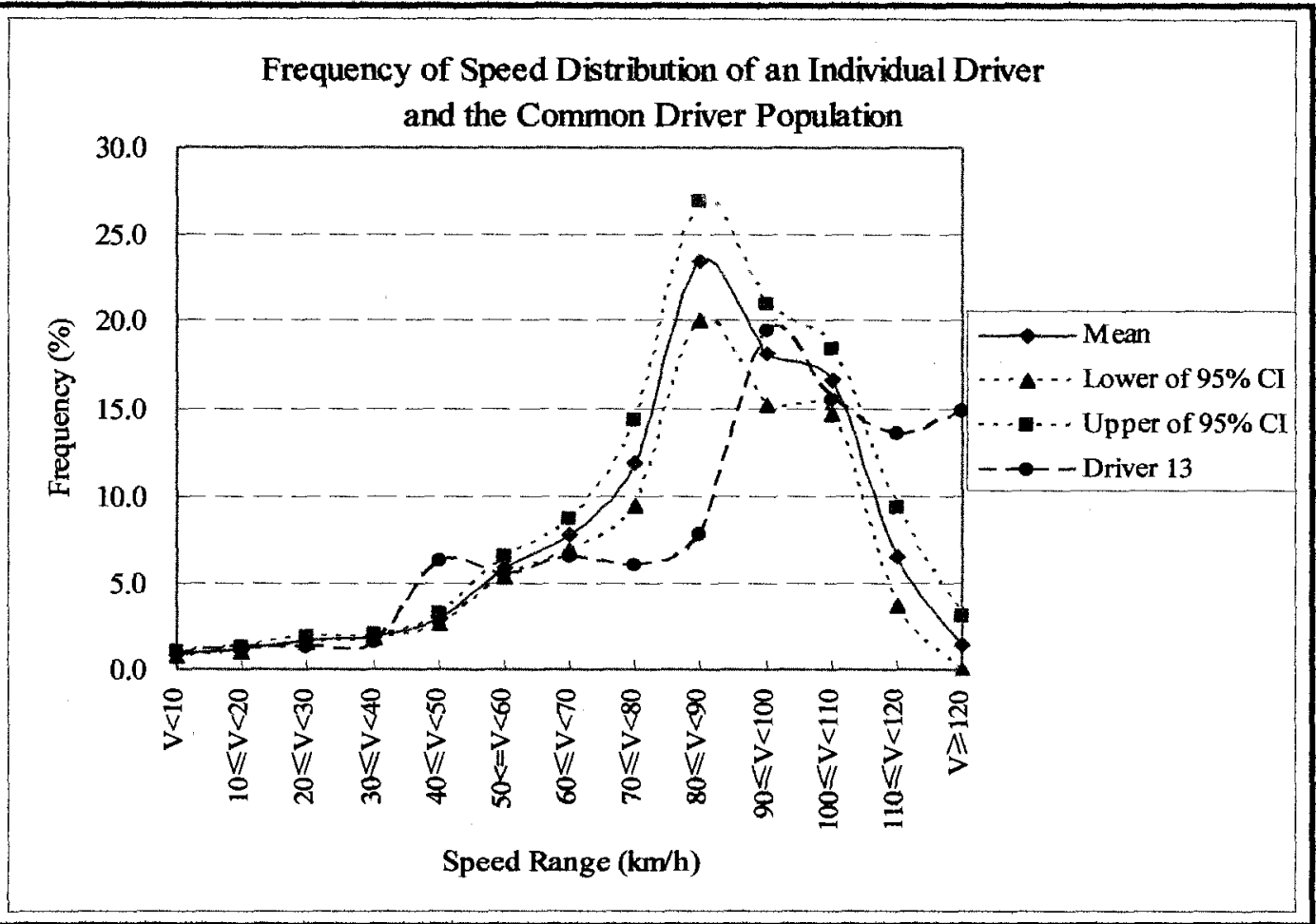

Figure B - 21: Speed Distribution of Driver 13 and the Common Driver Population.

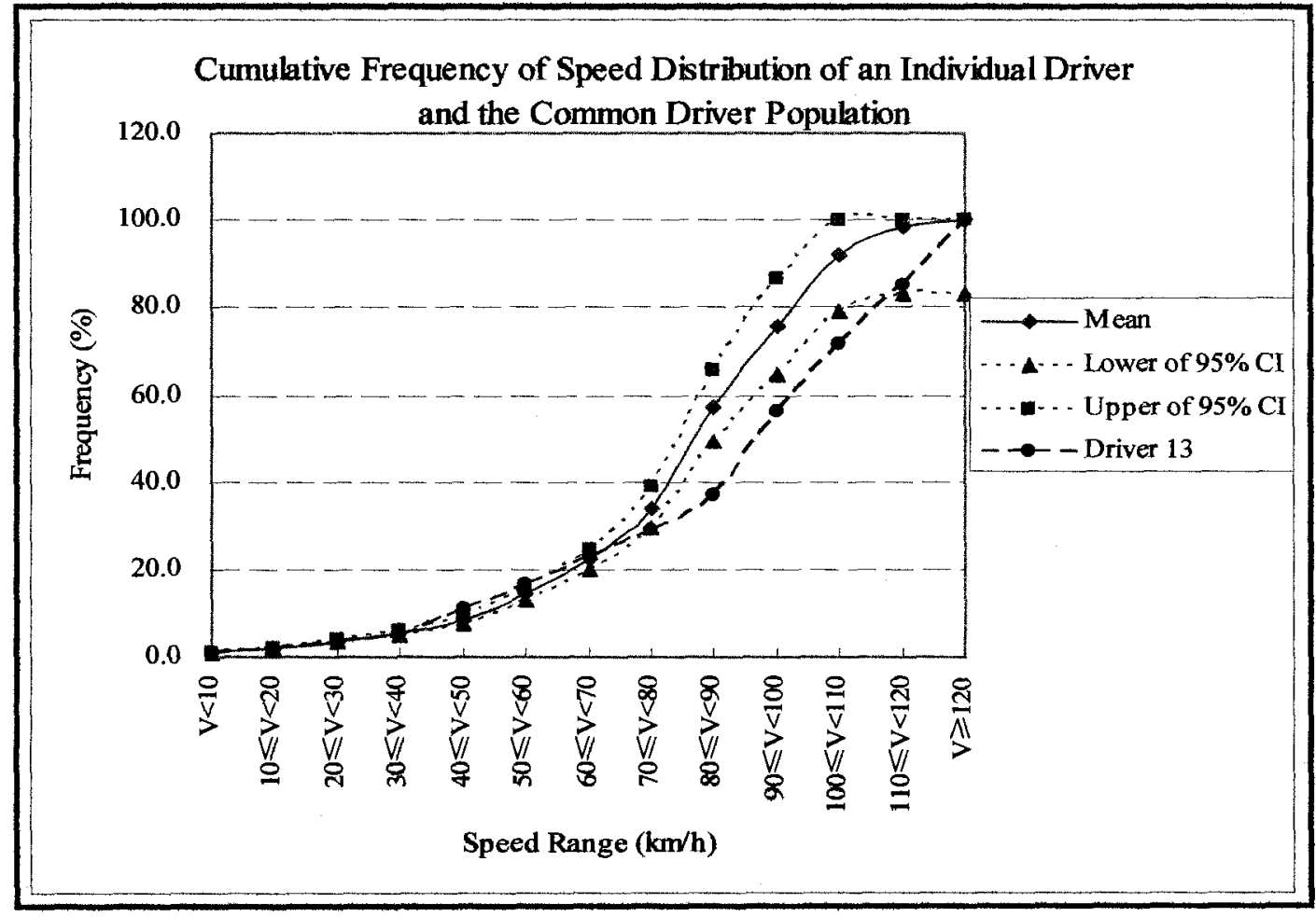

Figure B - 22: Cumulative Frequency of Speed Distribution of Driver 13 and the Common Driver Population. 


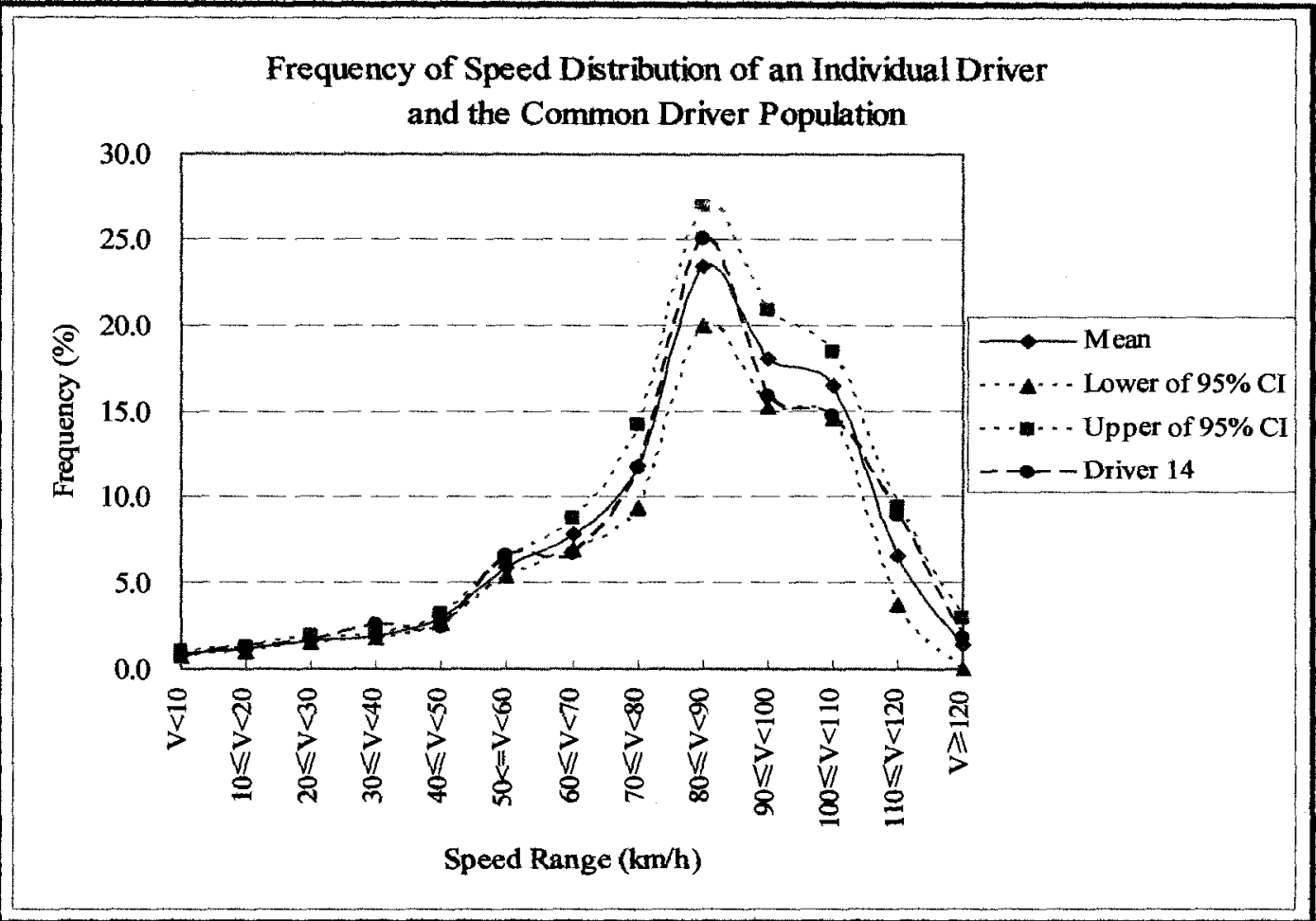

Figure B - 23: Speed Distribution of Driver 14 and the Common Driver Population.

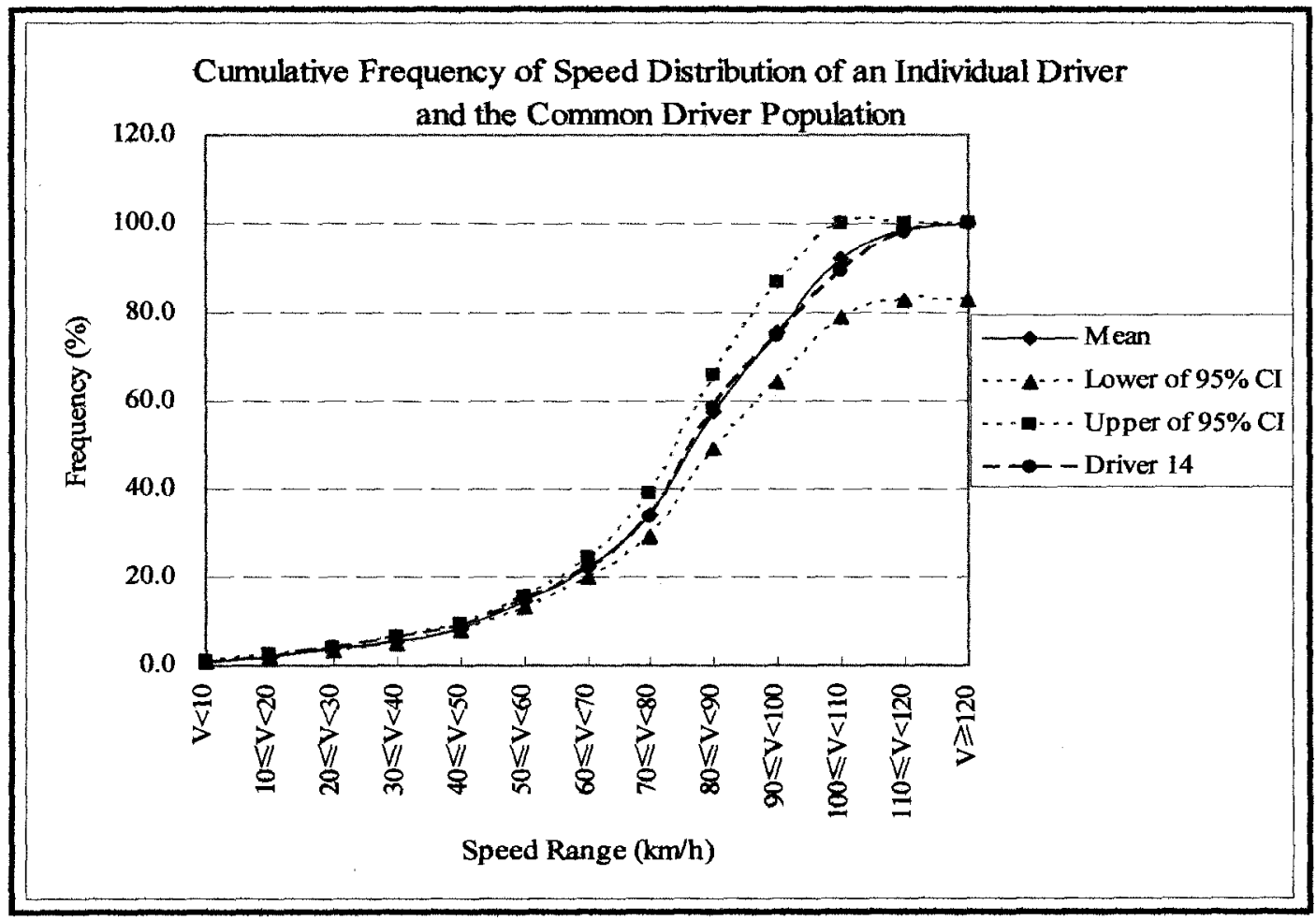

Figure B - 24: Cumulative Frequency of Speed Distribution of Driver 14 and the Common Driver Population. 


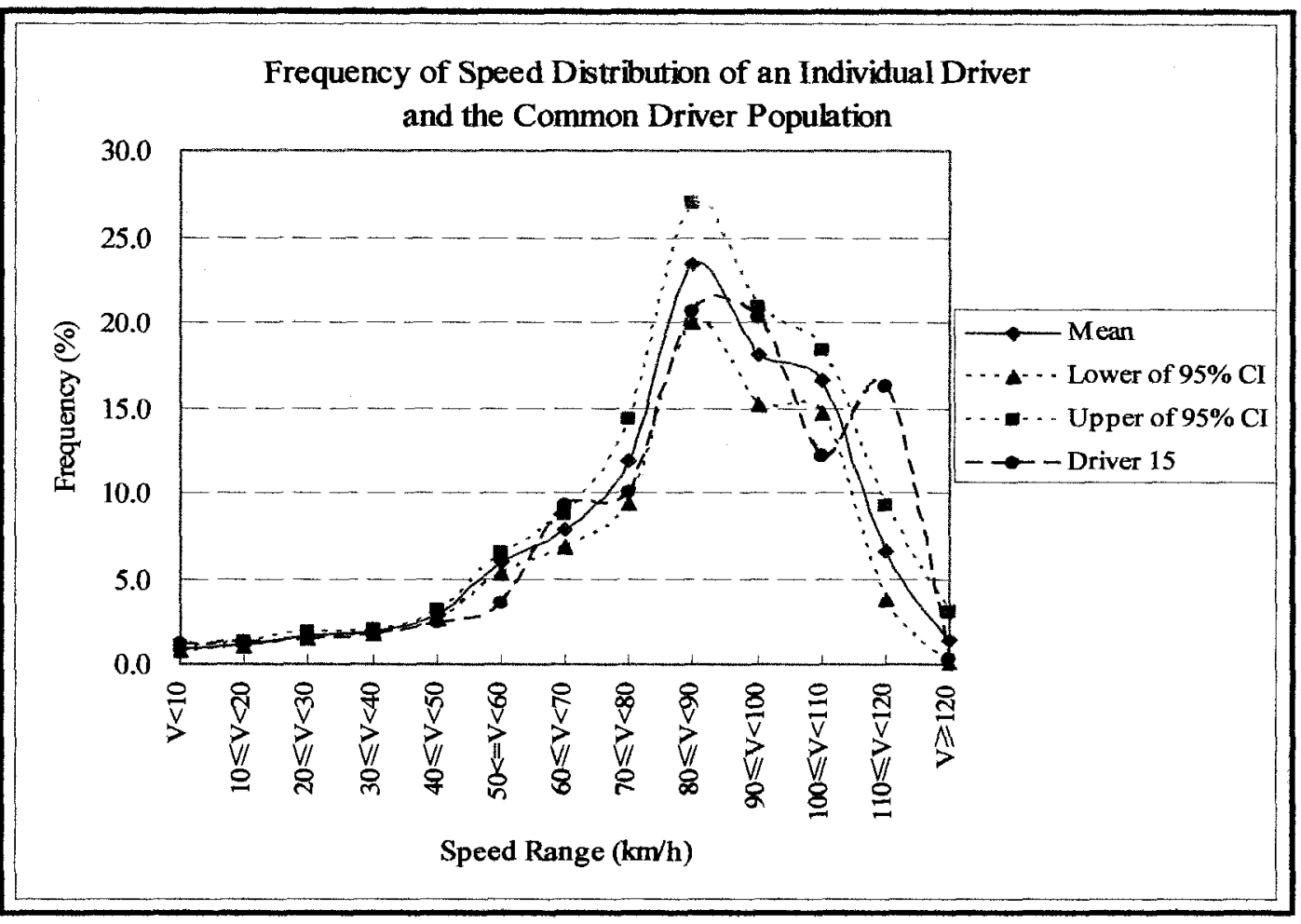

Figure B -25: Speed Distribution of Driver 15 and the Common Driver Population.

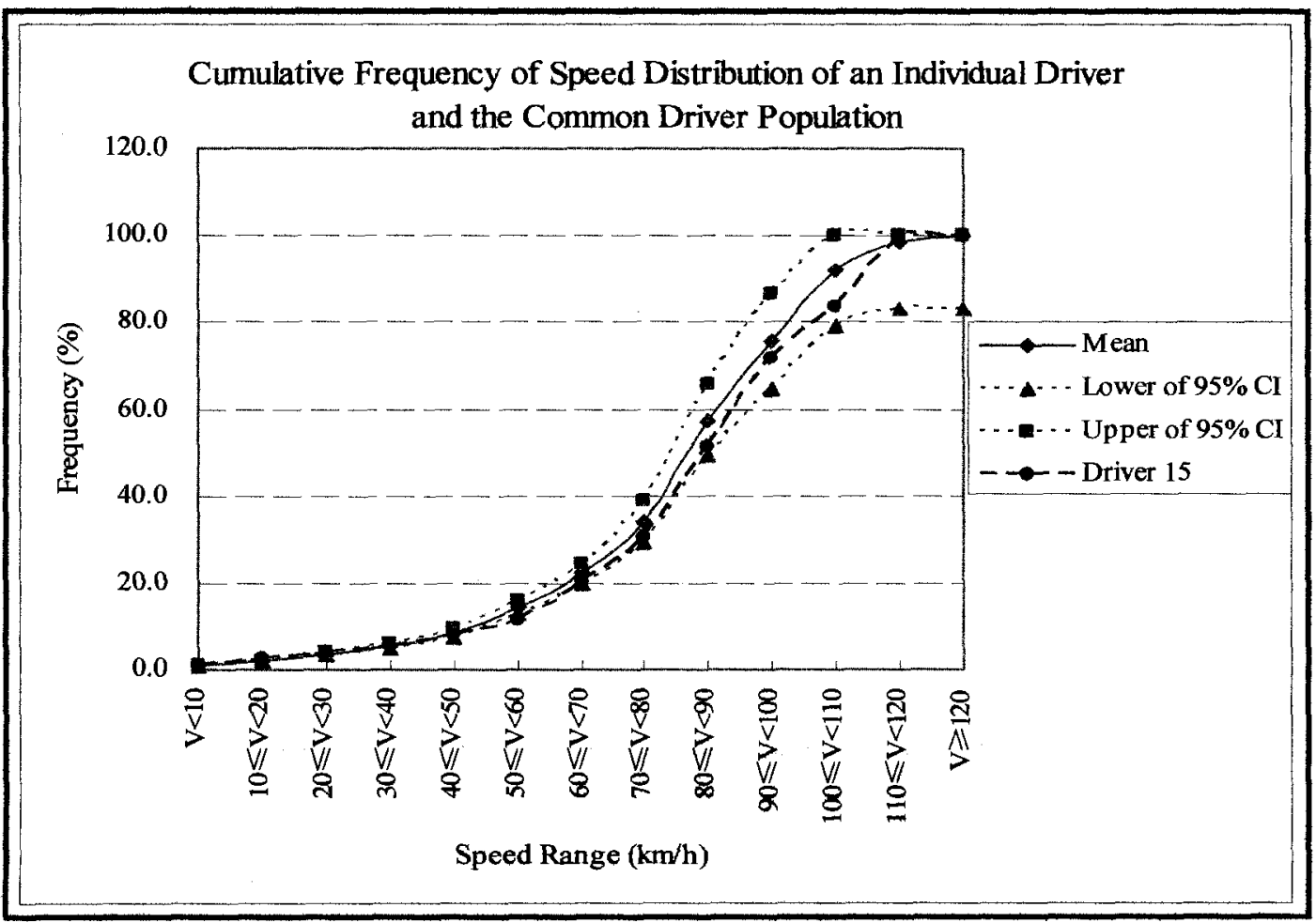

Figure B - 26: Cumulative Frequency of Speed Distribution of Driver 15 and the Common Driver Population. 


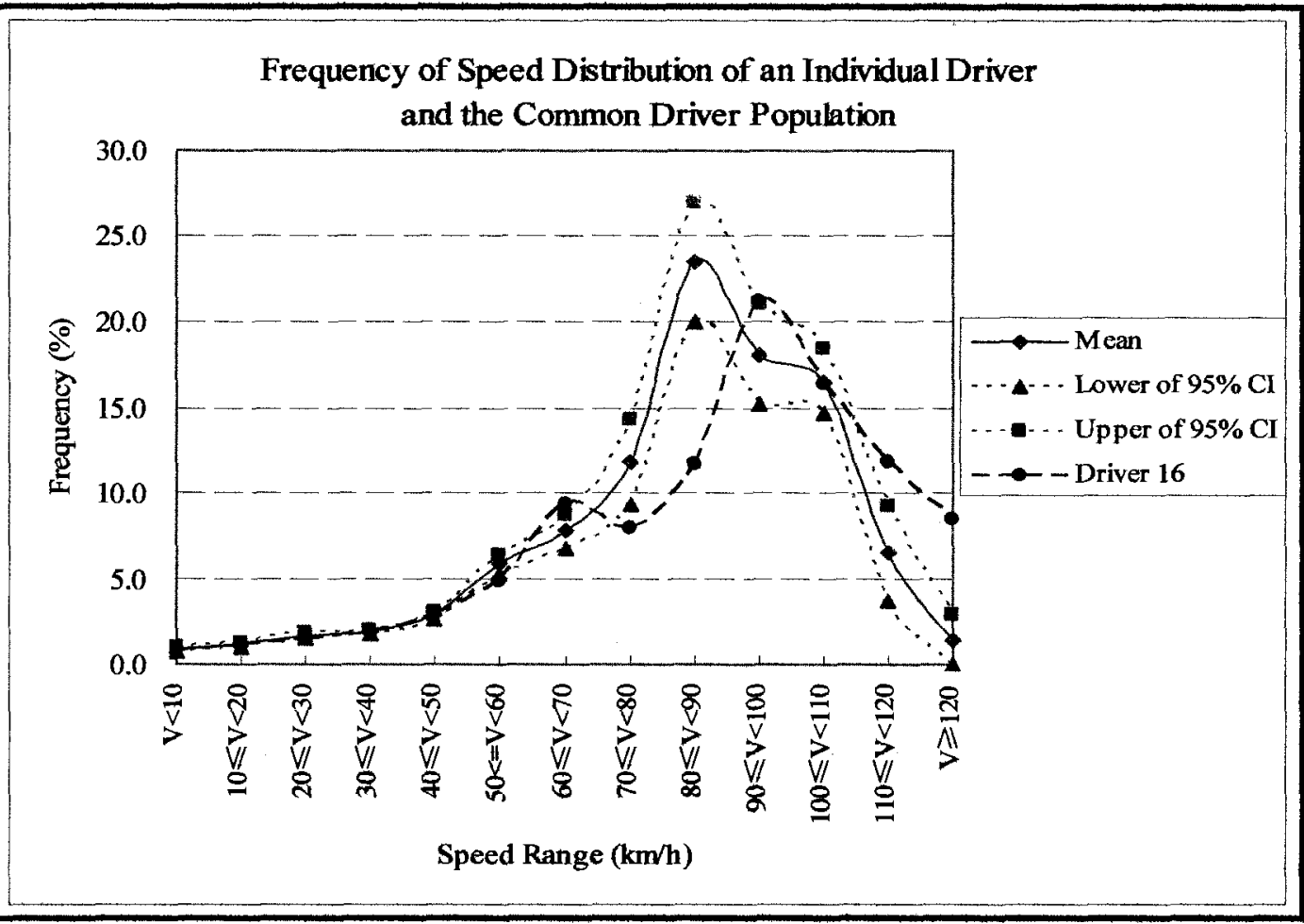

Figure B - 27: Speed Distribution of Driver 16 and the Common Driver Population.

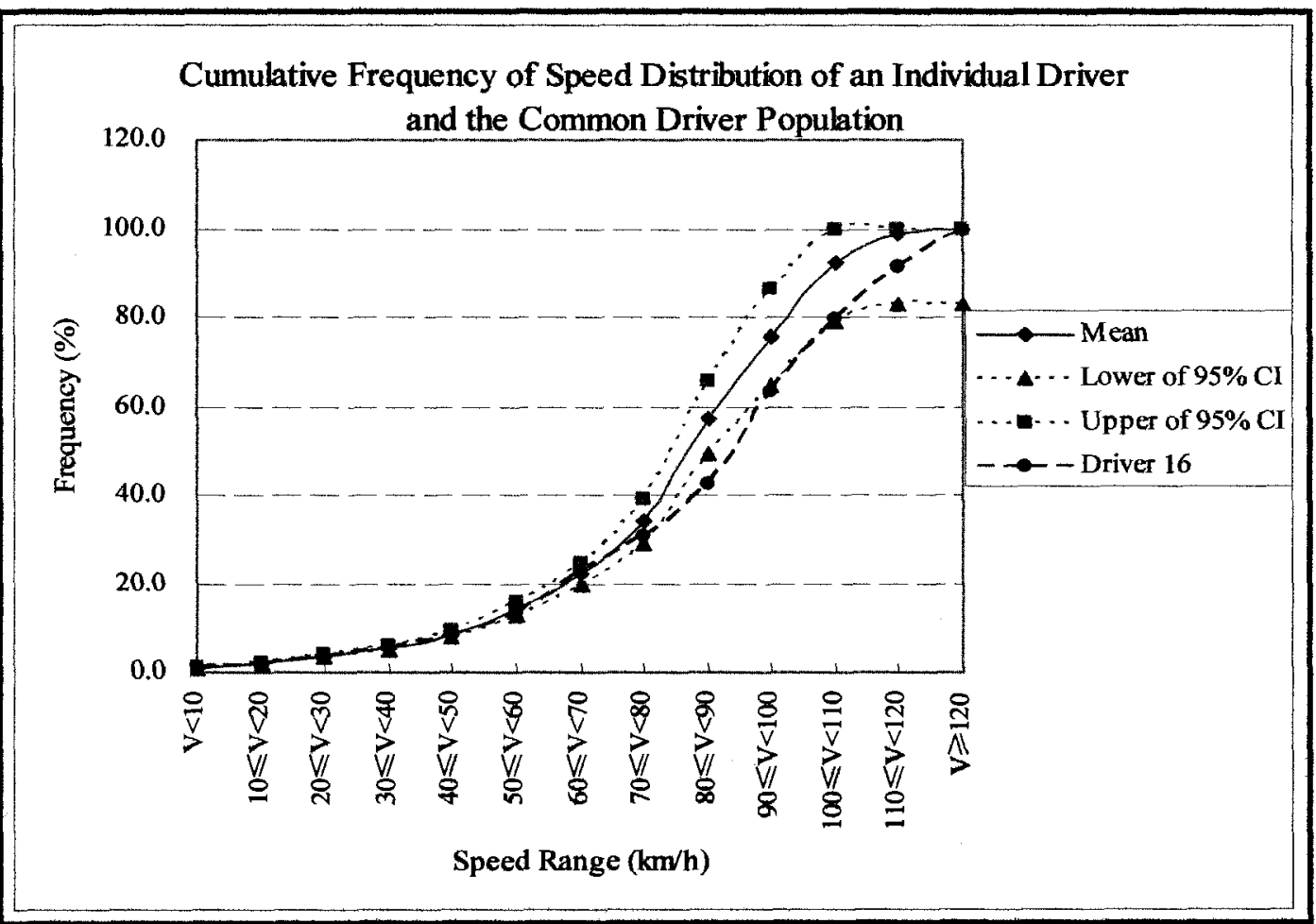

Figure B - 28: Cumulative Frequency of Speed Distribution of Driver 16 and the Common Driver Population. 


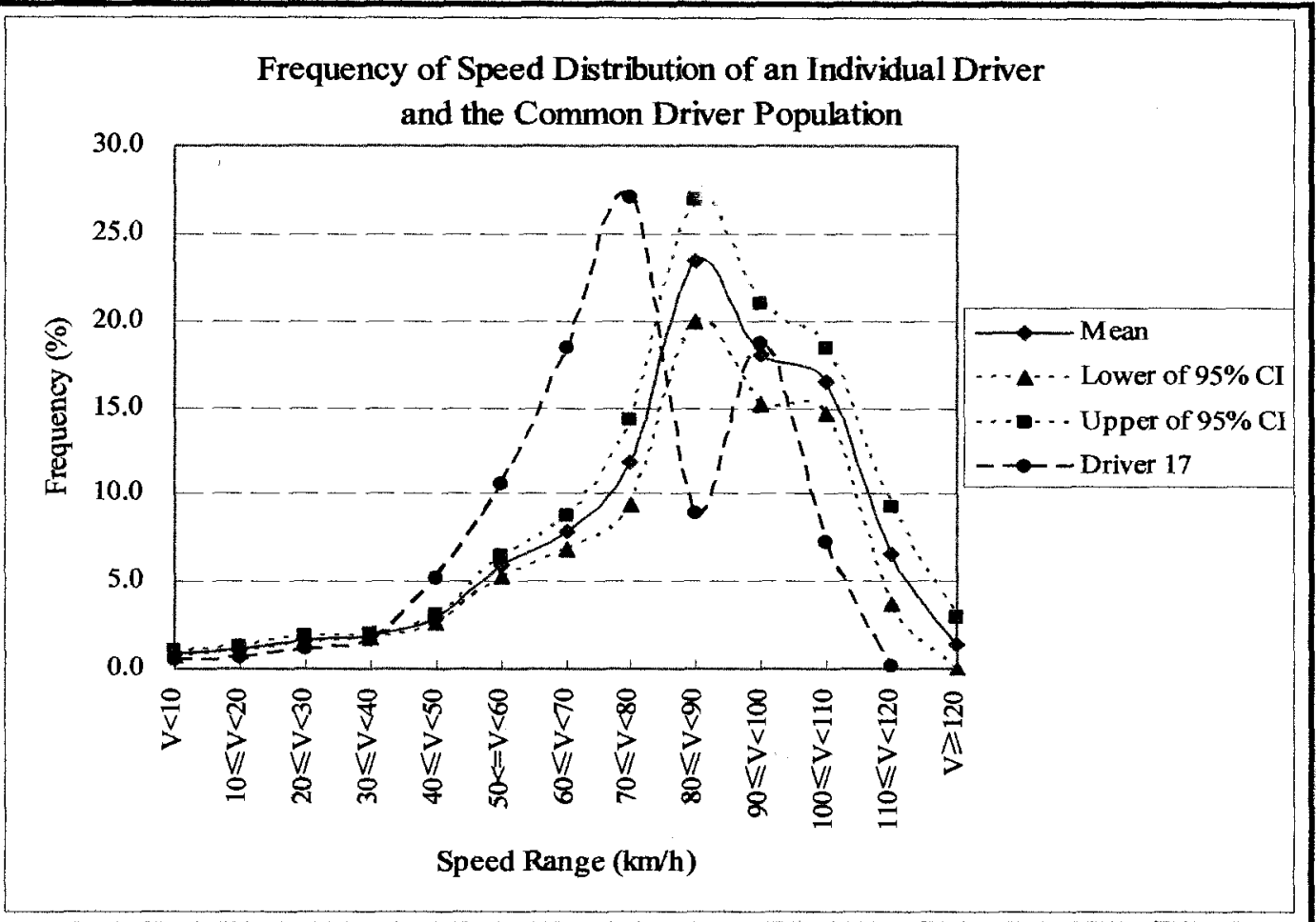

Figure B - 29: Speed Distribution of Driver 17 and the Common Driver Population.

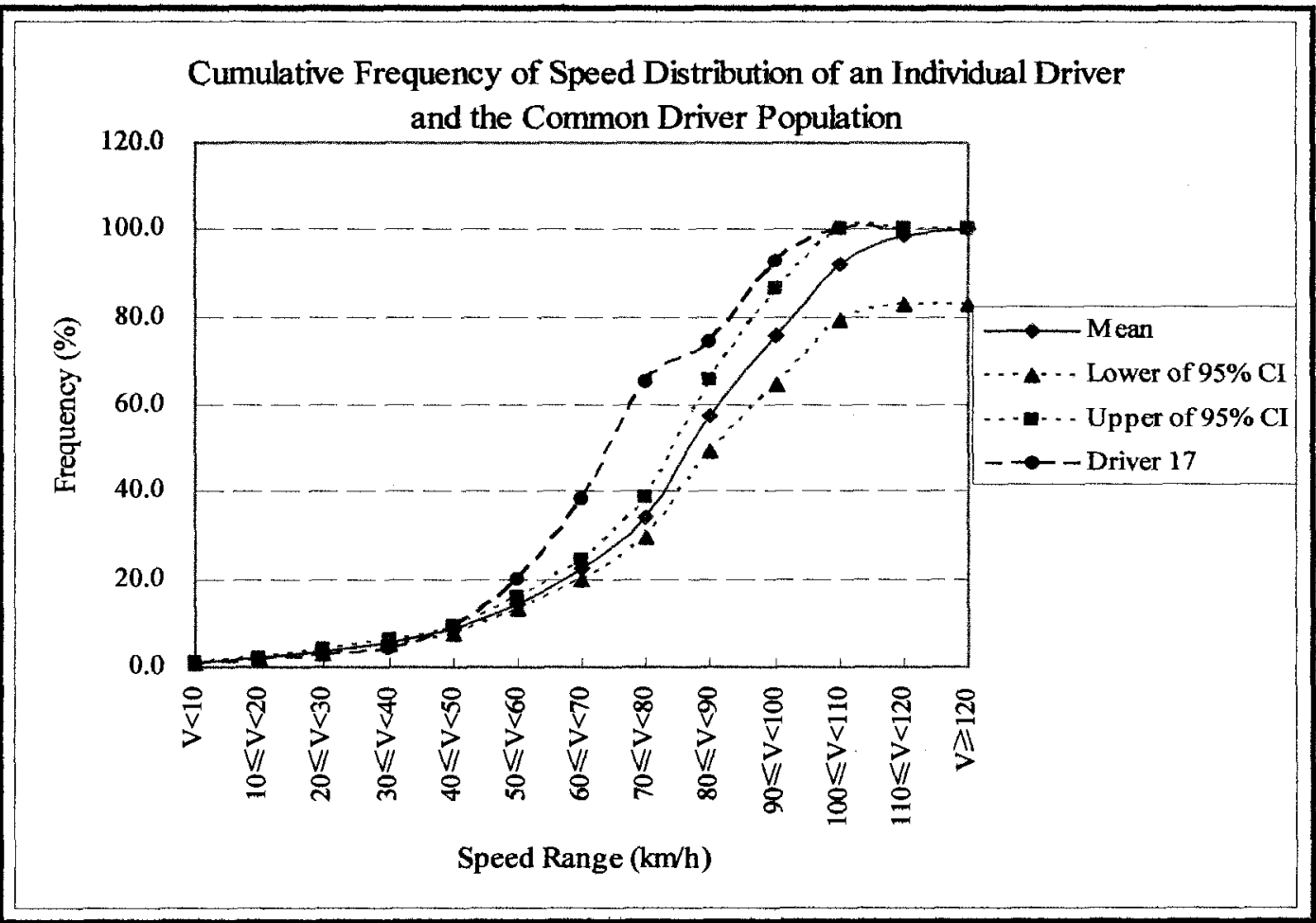

Figure B - 30: Cumulative Frequency of Speed Distribution of Driver 17 and the Common Driver Population. 


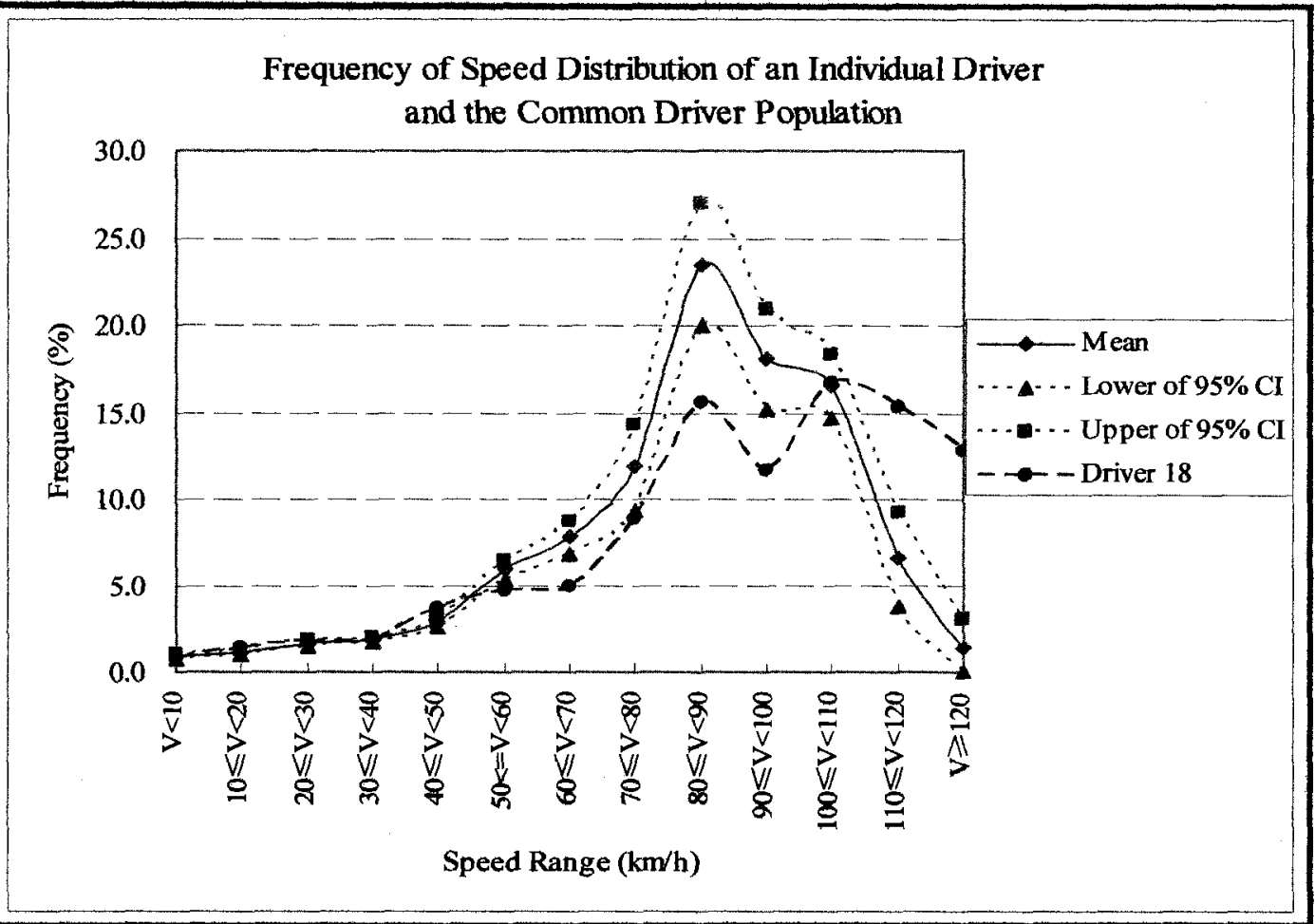

Figure B - 31: Speed Distribution of Driver 18 and the Common Driver Population.

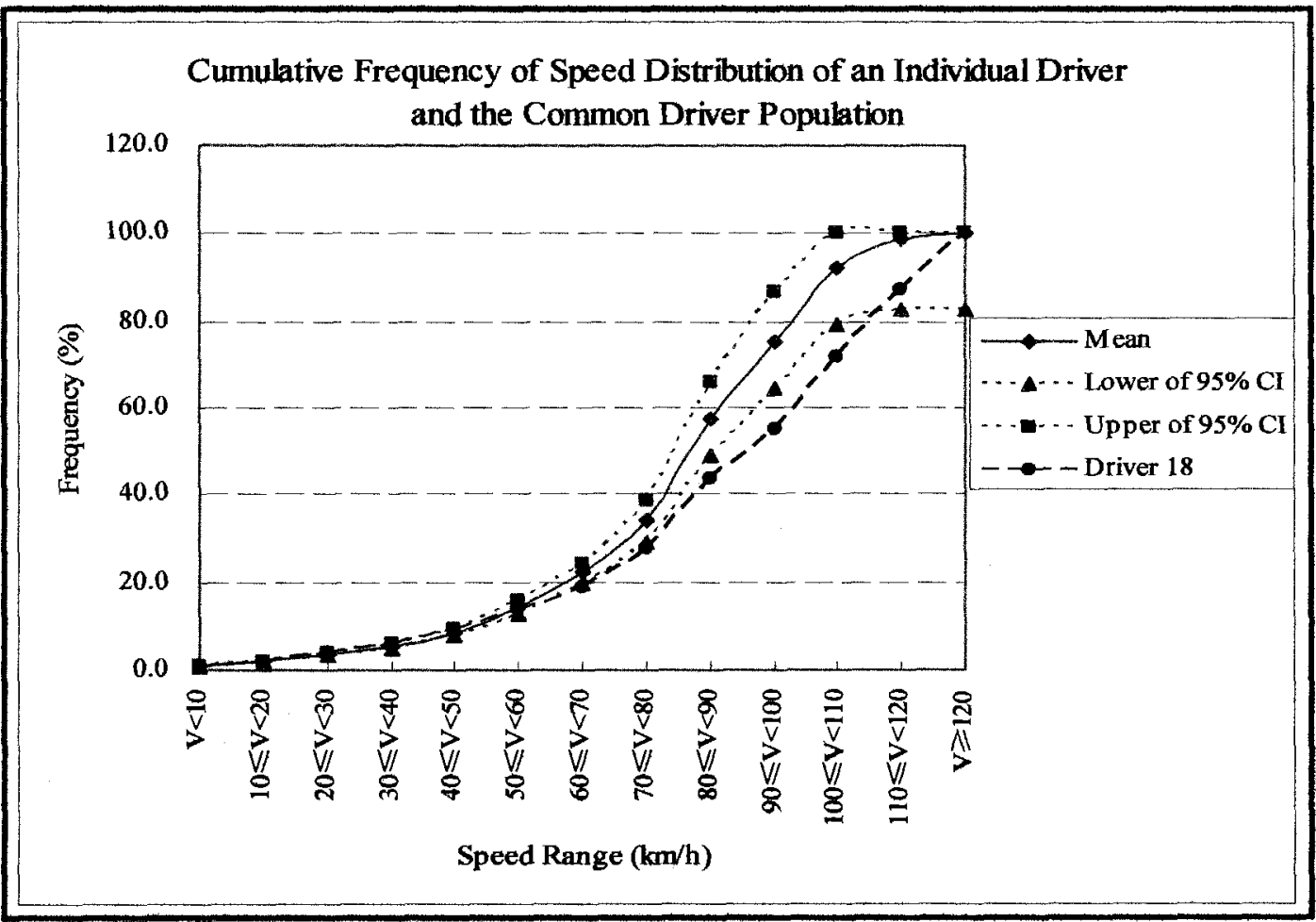

Figure B - 32: Cumulative Frequency of Speed Distribution of Driver 18 and the Common Driver Population. 


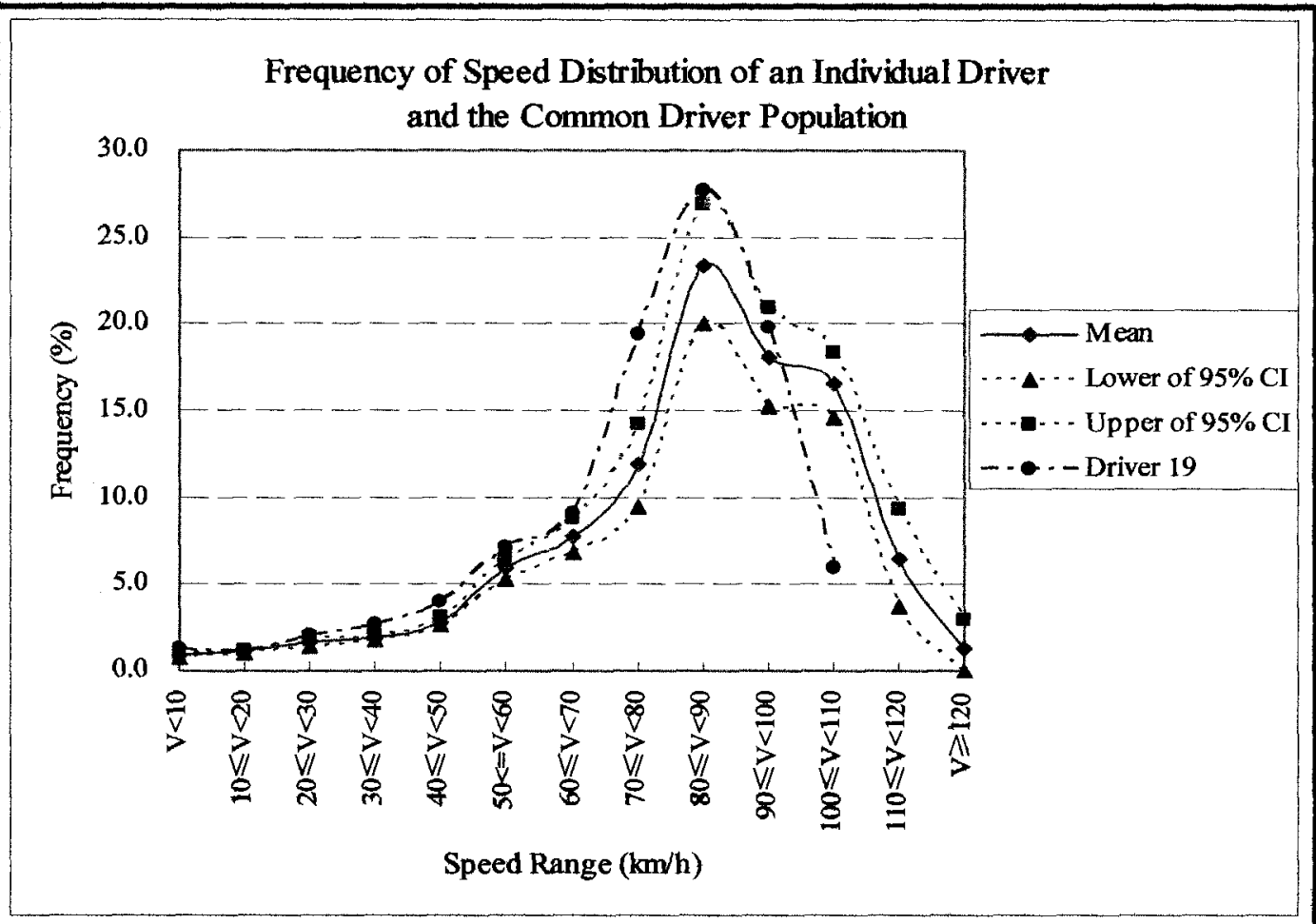

Figure B - 33: Speed Distribution of Driver 19 and the Common Driver Population.

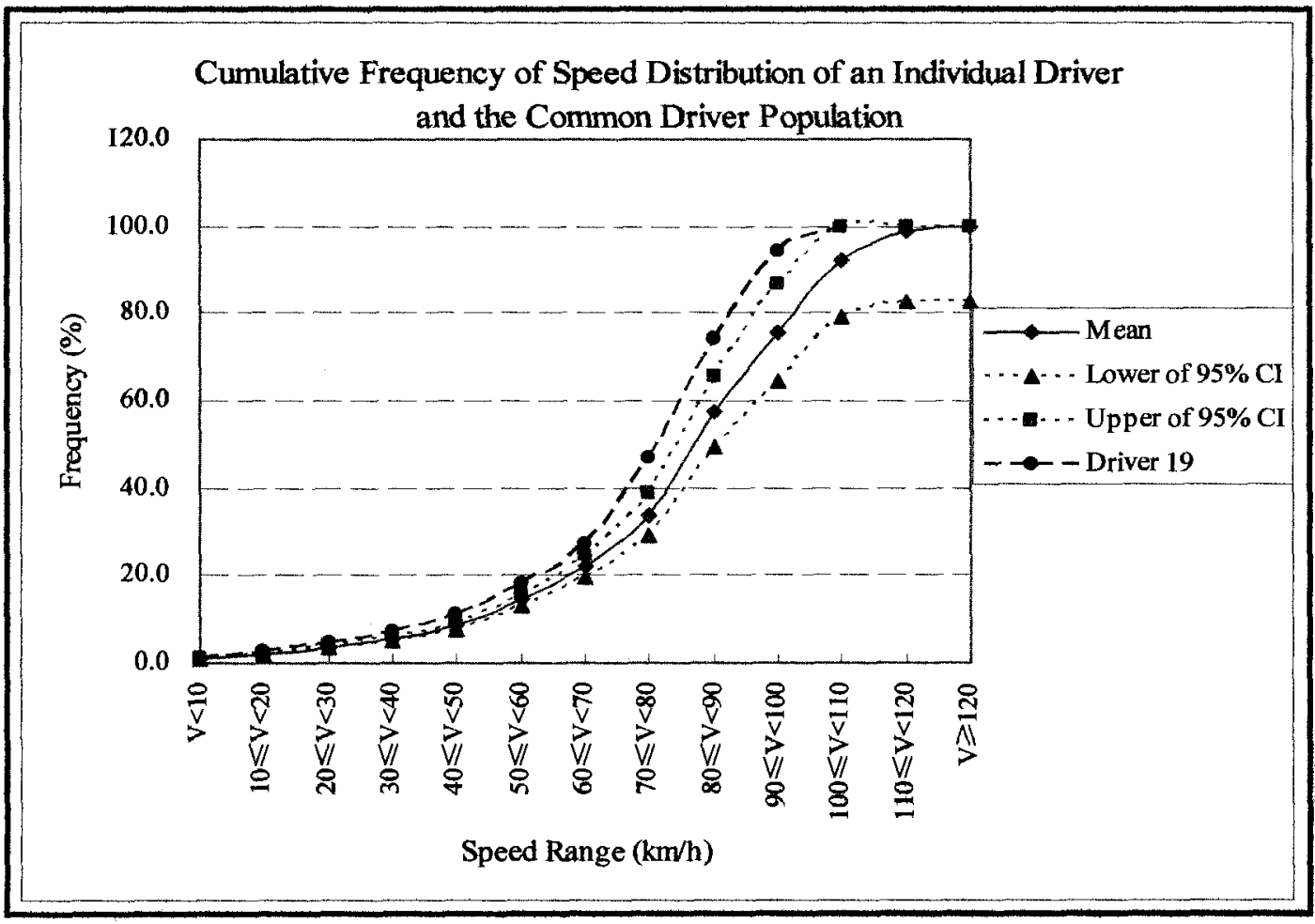

Figure B - 34: Cumulative Frequency of Speed Distribution of Driver 19 and the Common Driver Population. 


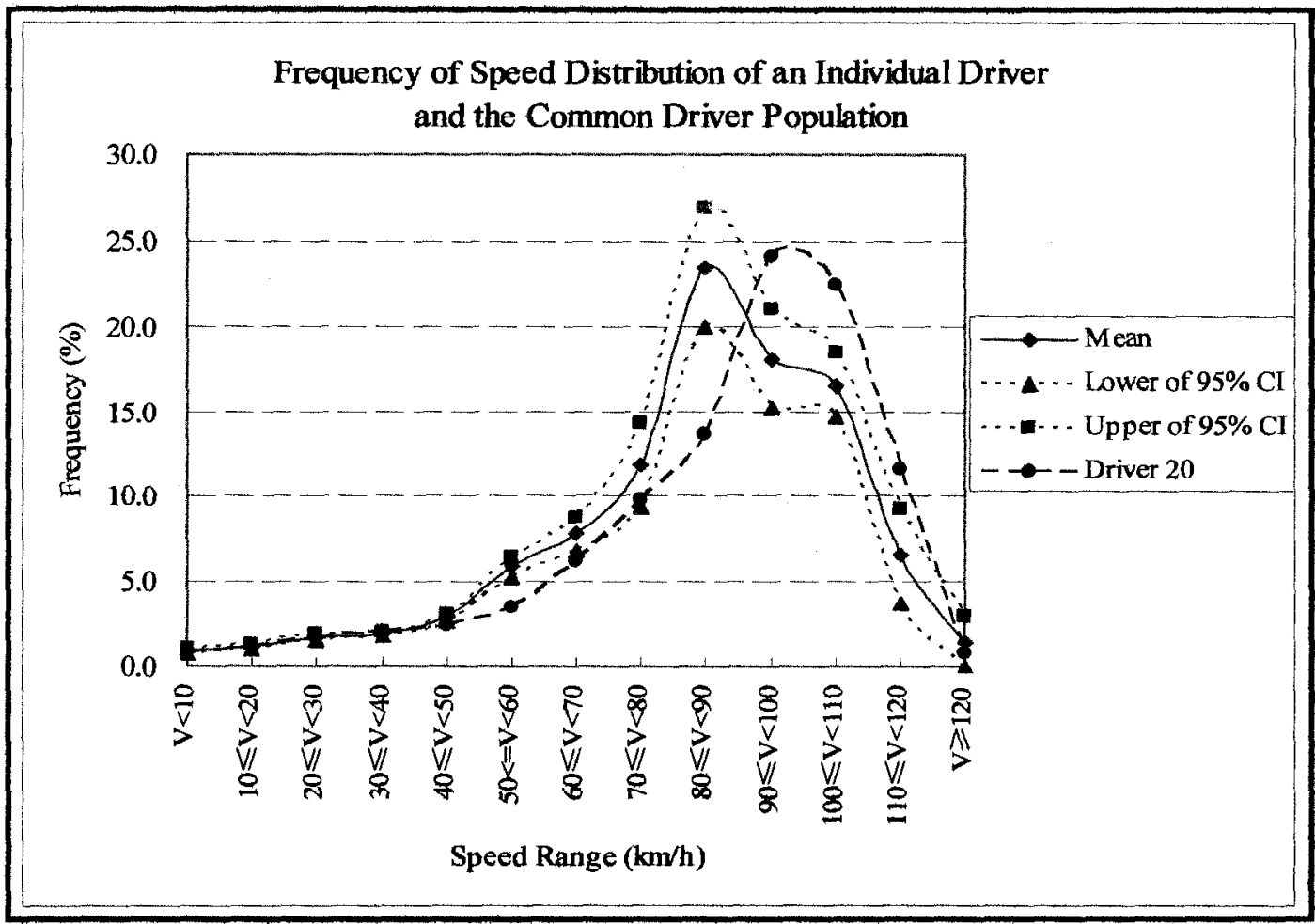

Figure B - 35: Speed Distribution of Driver 20 and the Common Driver Population.

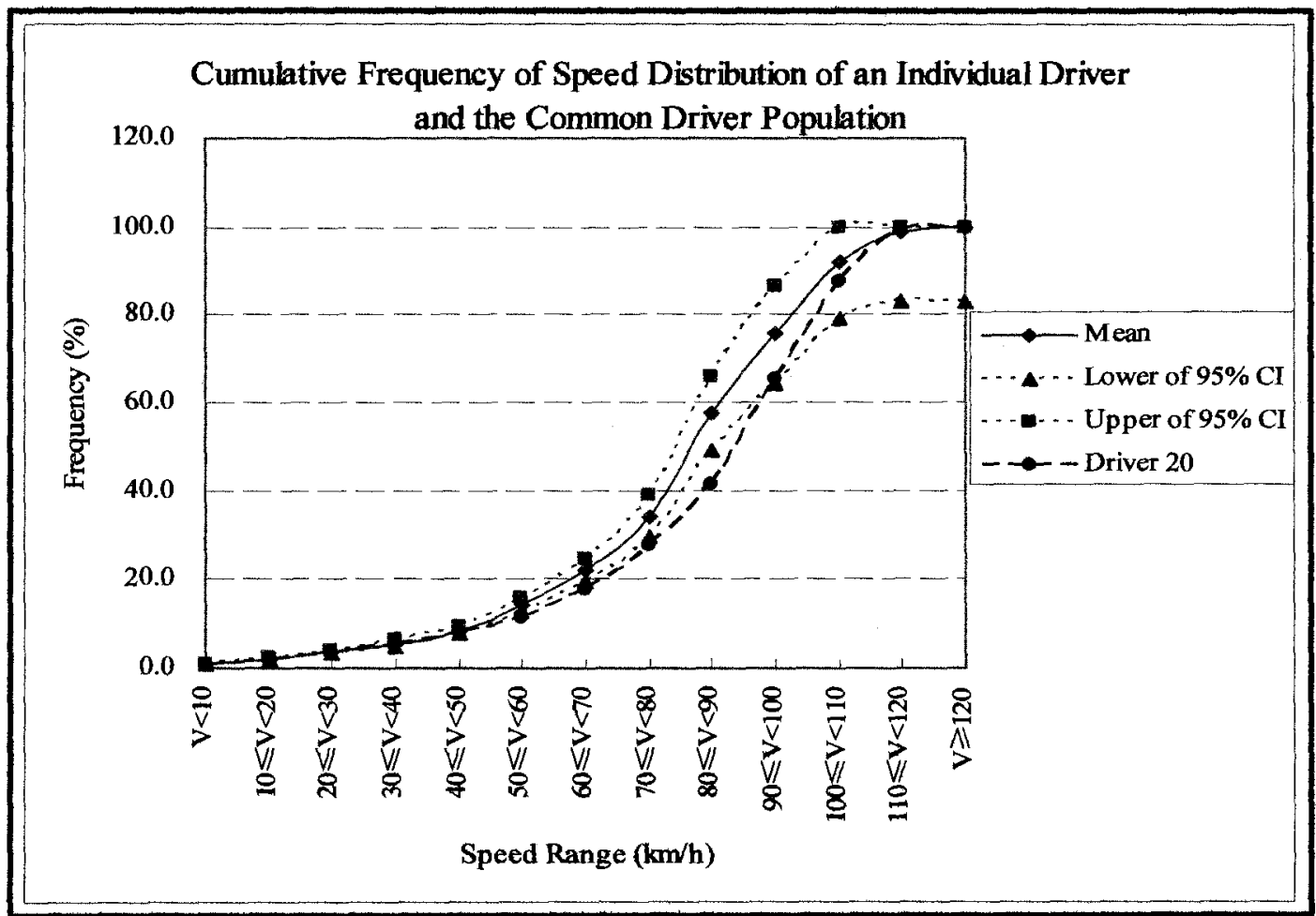

Figure B - 36: Cumulative Frequency of Speed Distribution of Driver 20 and the Common Driver Population. 


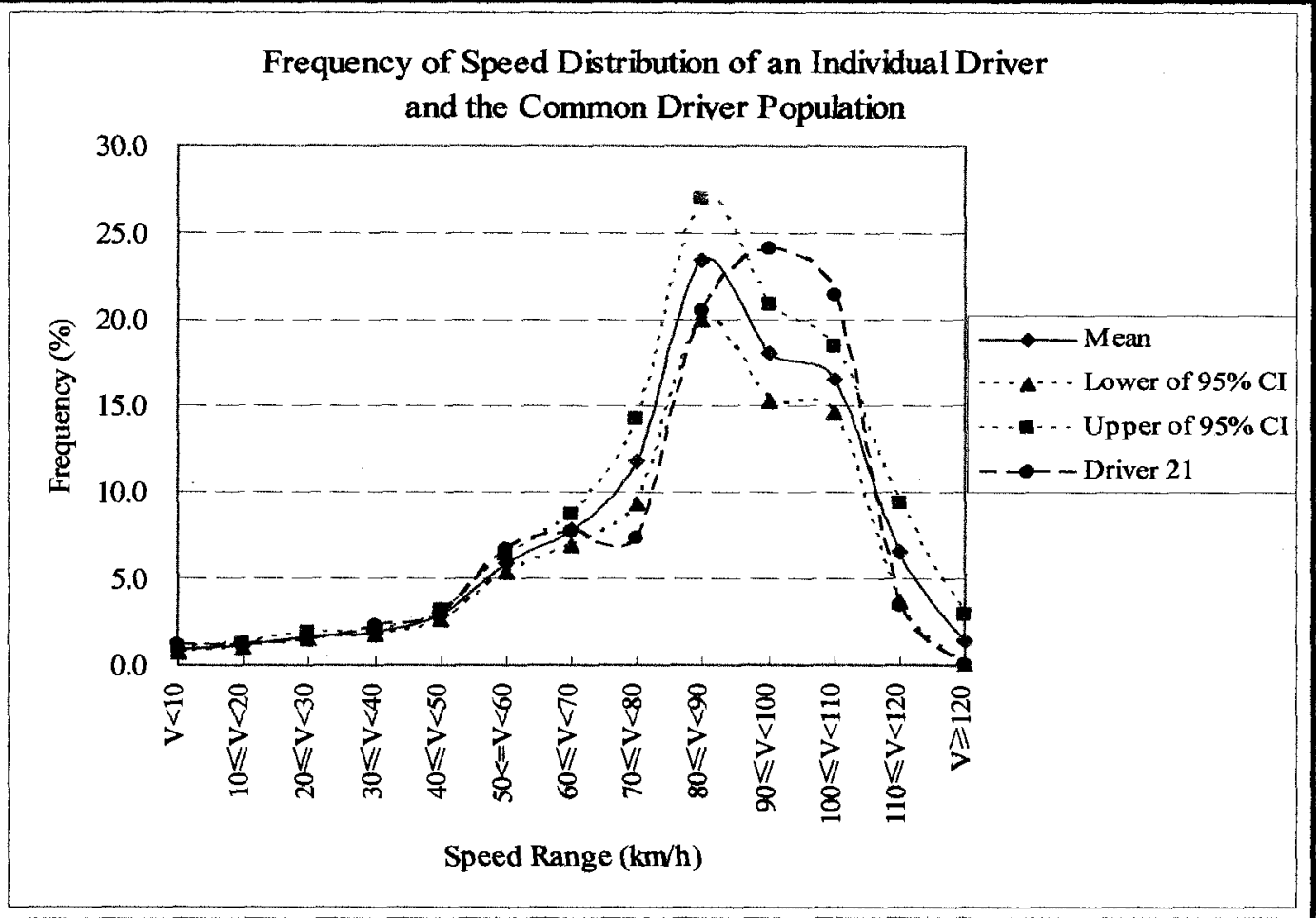

Figure B - 37: Speed Distribution of Driver 21 and the Common Driver Population.

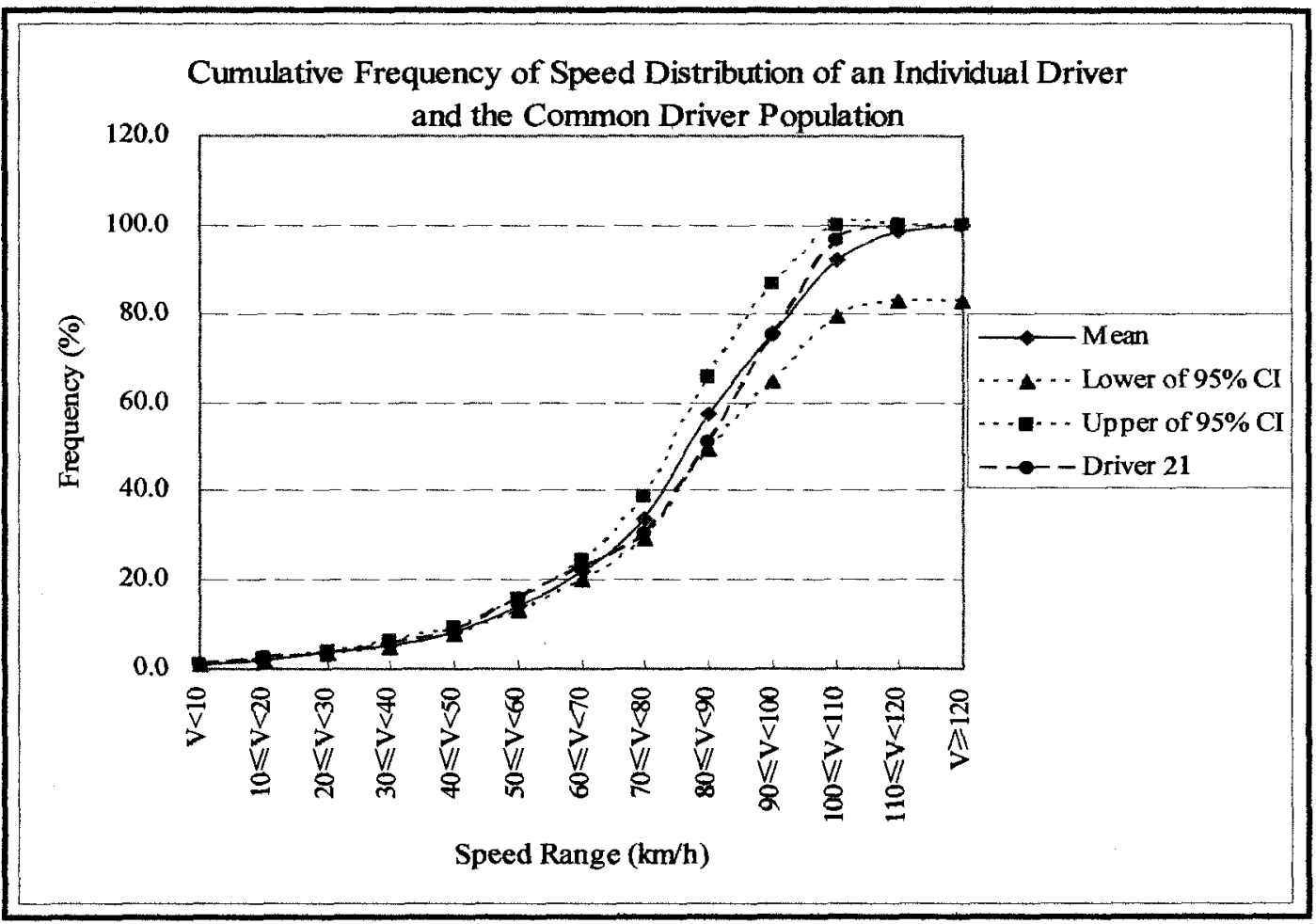

Figure B - 38: Cumulative Frequency of Speed Distribution of Driver 21 and the Common Driver Population. 


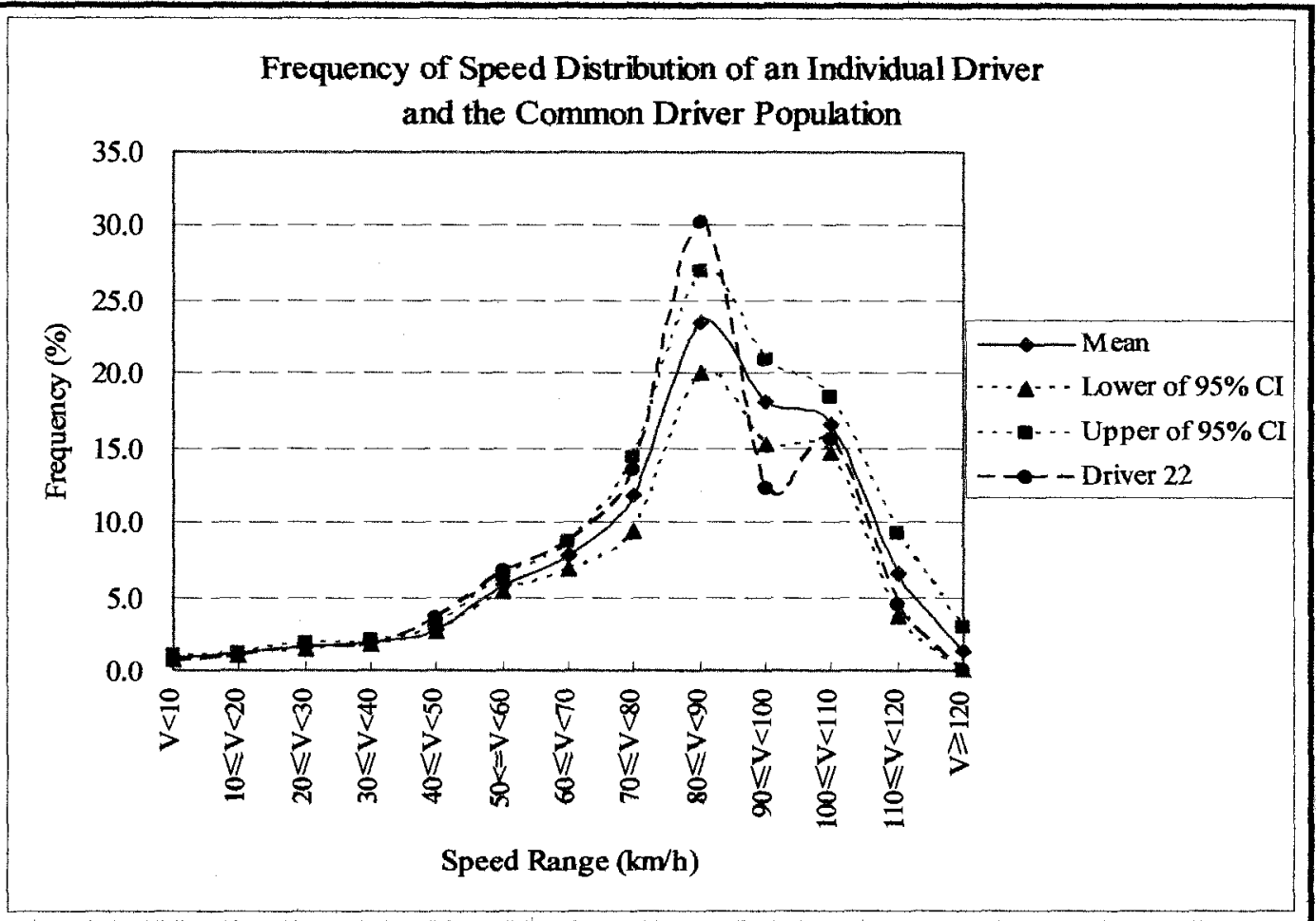

Figure B - 39: Speed Distribution of Driver 22 and the Common Driver Population.

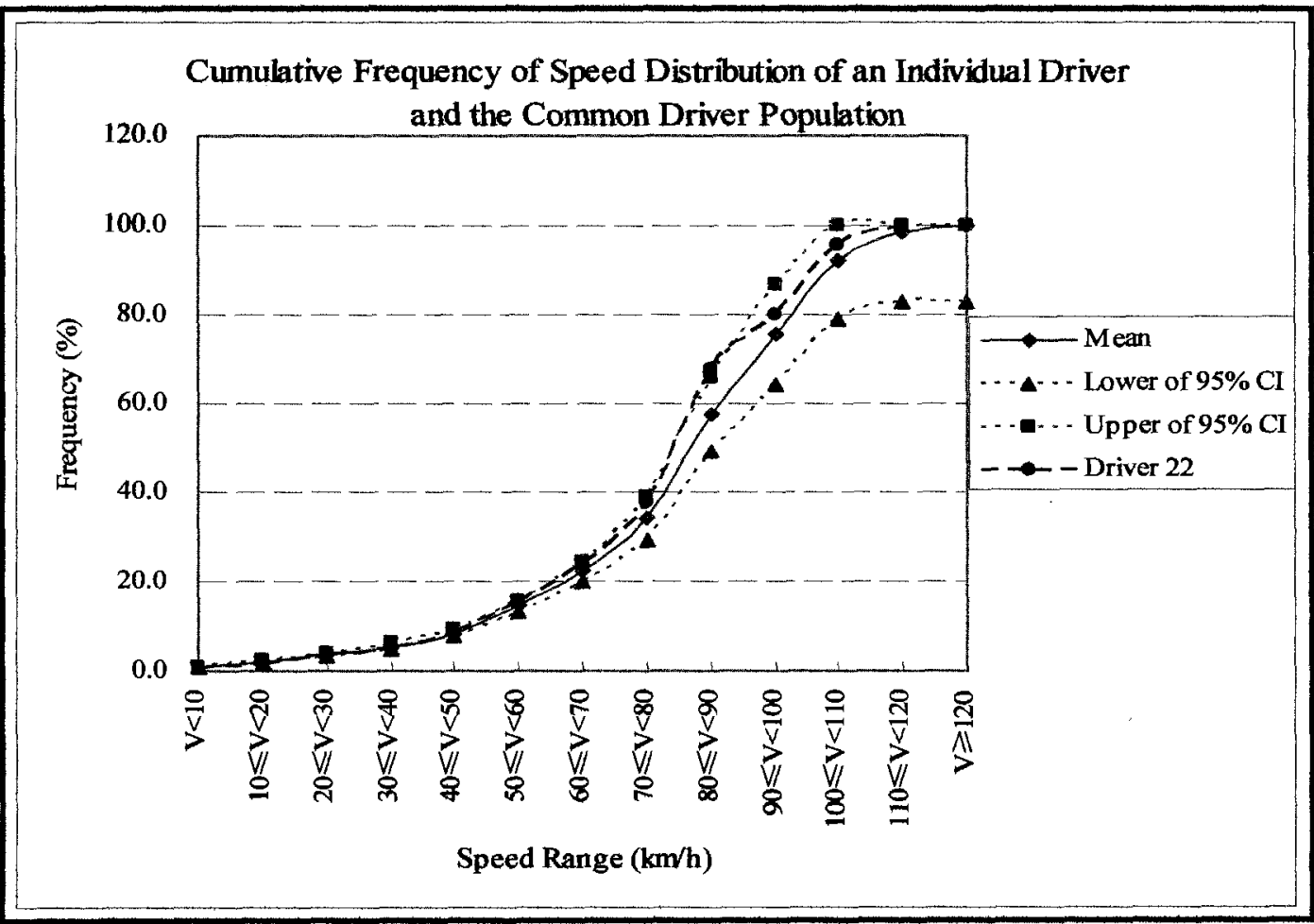

Figure B - 40: Cumulative Frequency of Speed Distribution of Driver 22 and the Common Driver Population. 


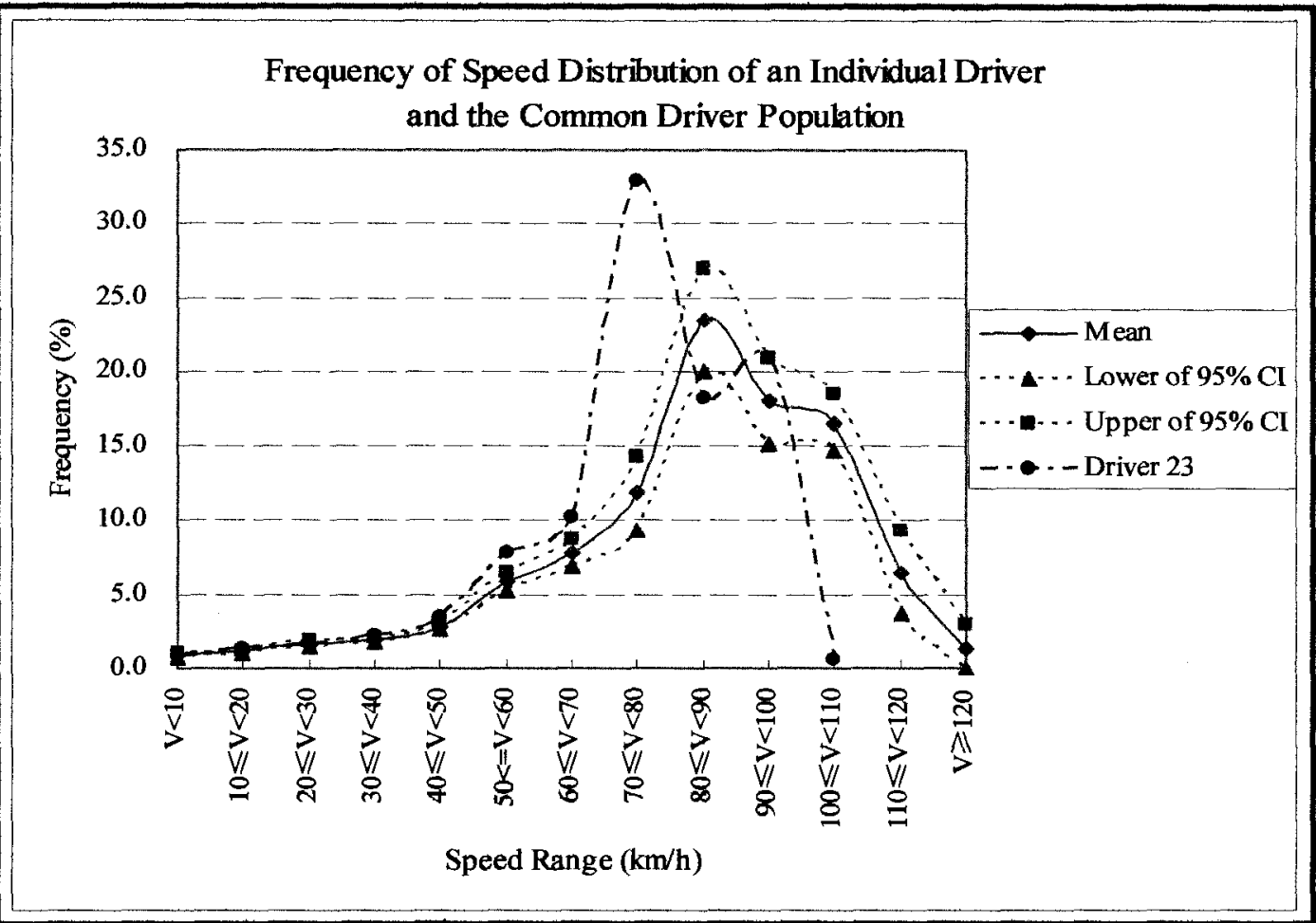

Figure B - 41: Speed Distribution of Driver 23 and the Common Driver Population.

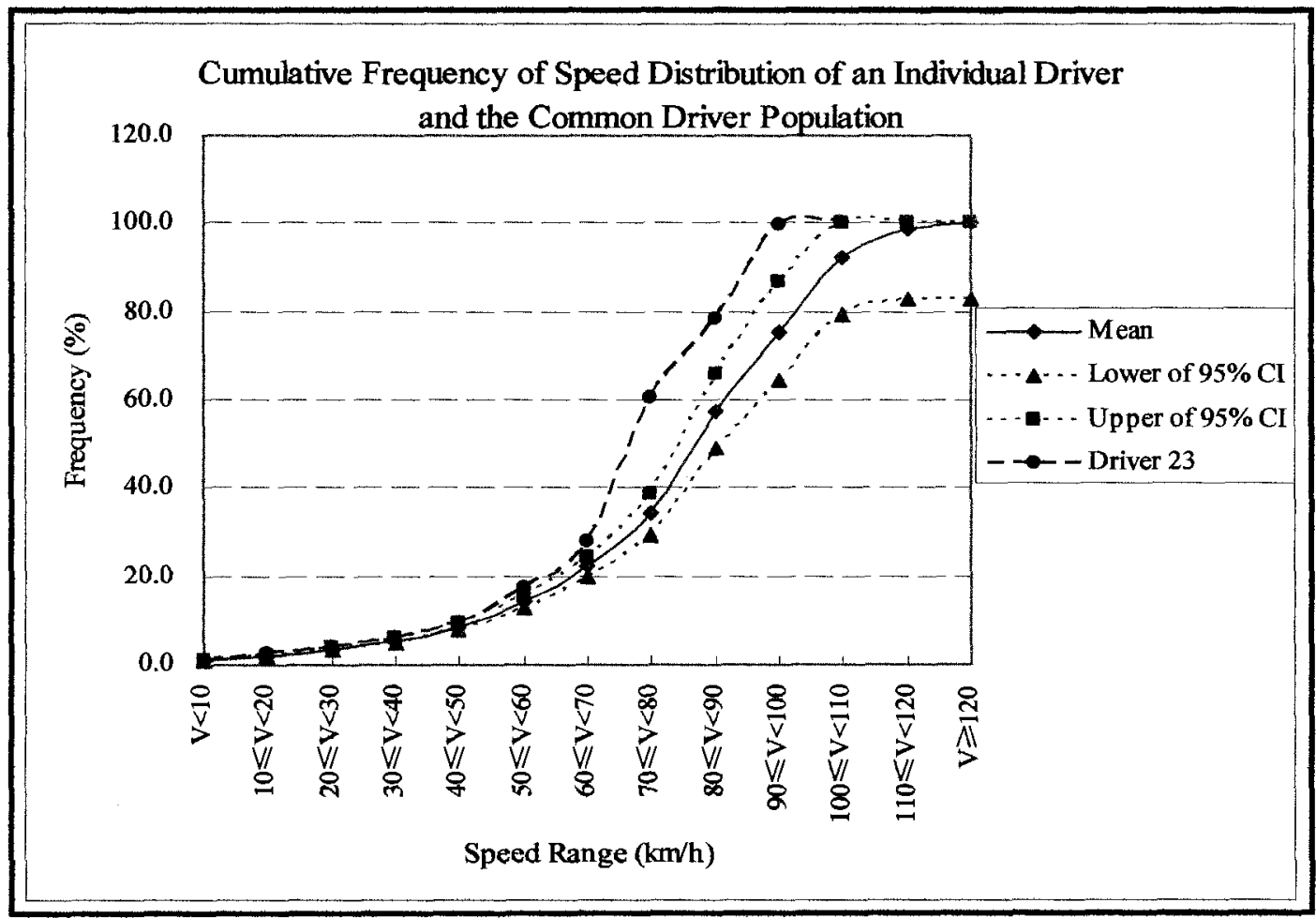

Figure B - 42: Cumulative Frequency of Speed Distribution of Driver 23 and the Common Driver Population. 


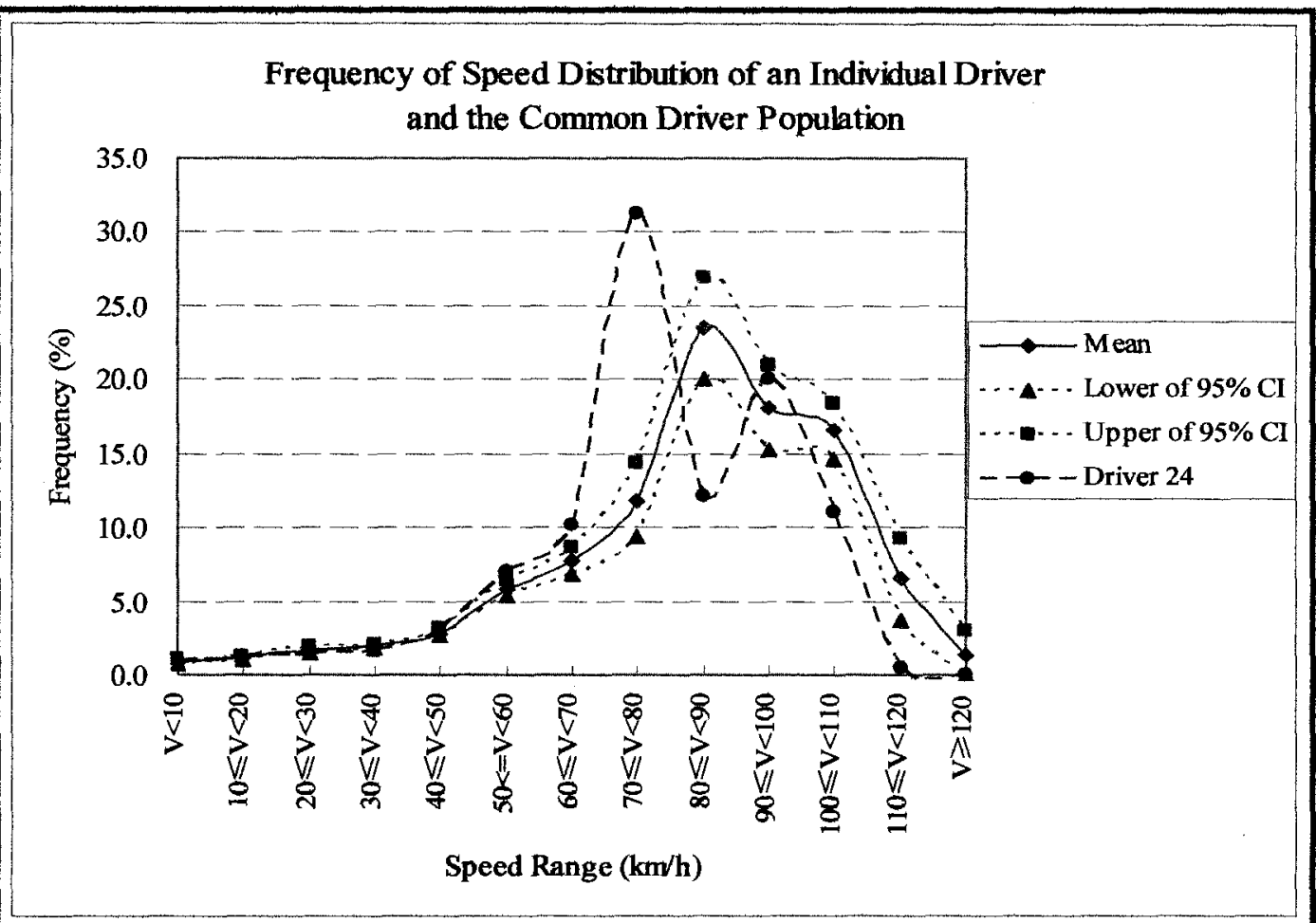

Figure B - 43: Speed Distribution of Driver 24 and the Common Driver Population.

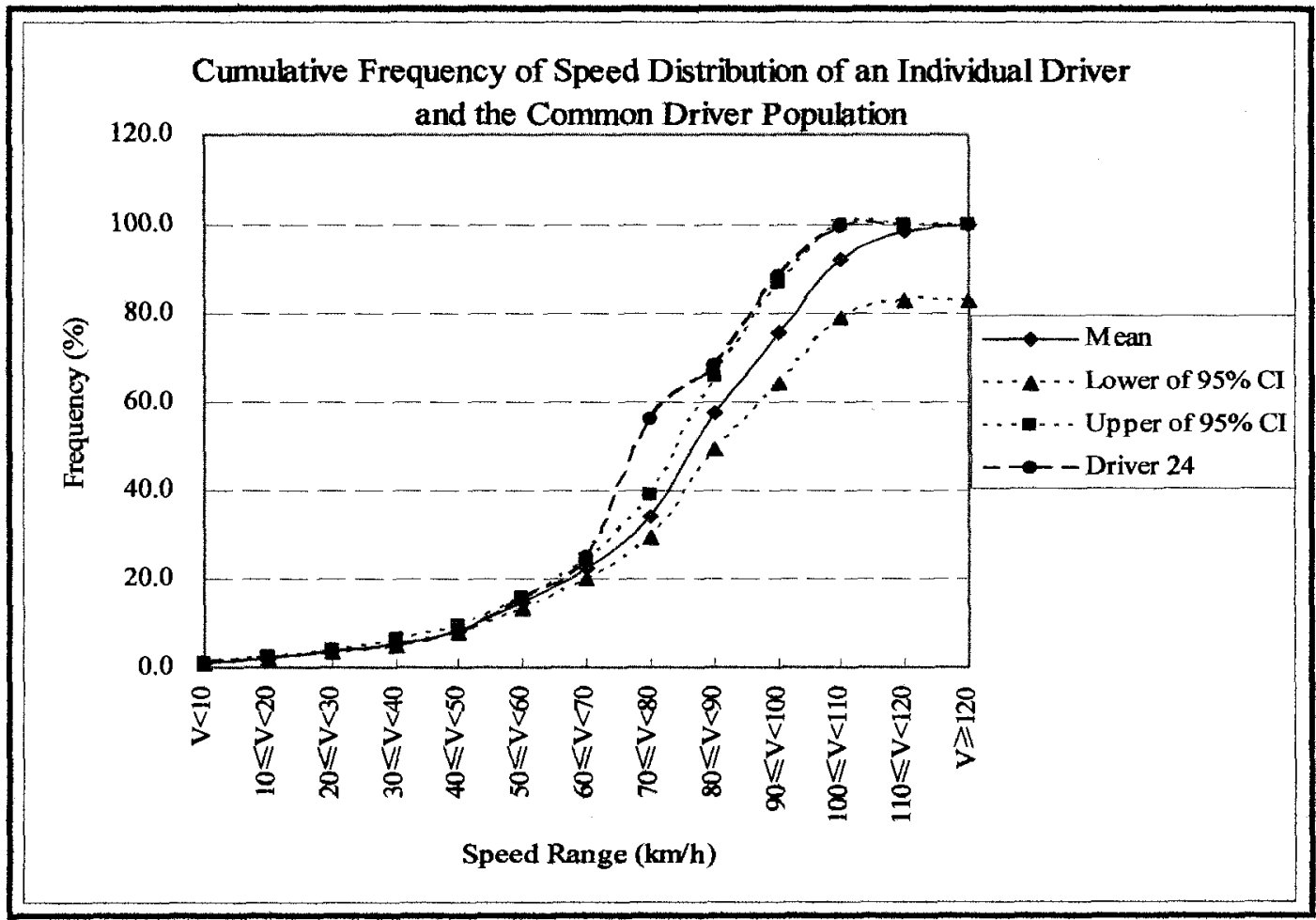

Figure B - 44: Cumulative Frequency of Speed Distribution of Driver 24 and the Common Driver Population. 


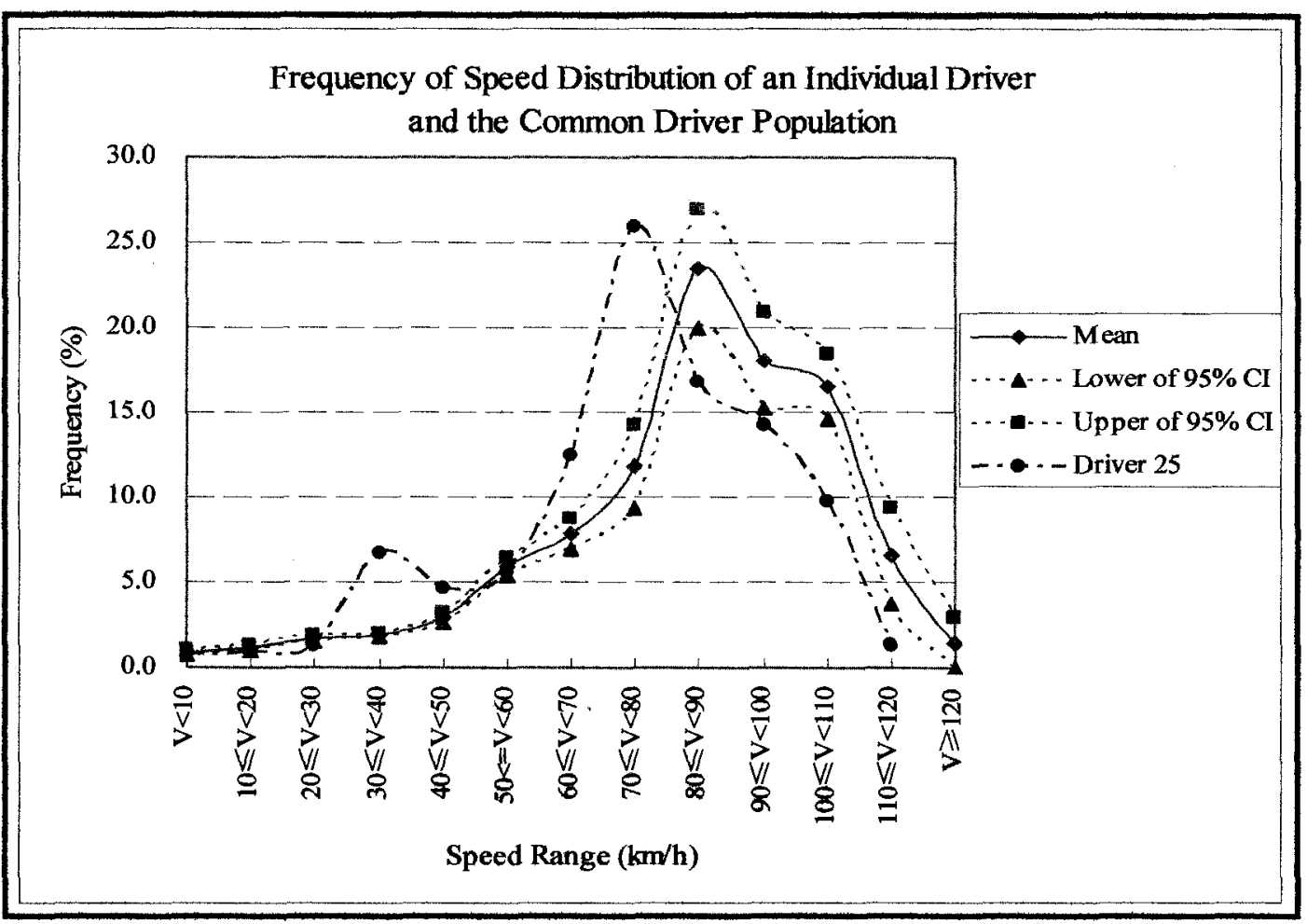

Figure B - 45: Speed Distribution of Driver 25 and the Common Driver Population.

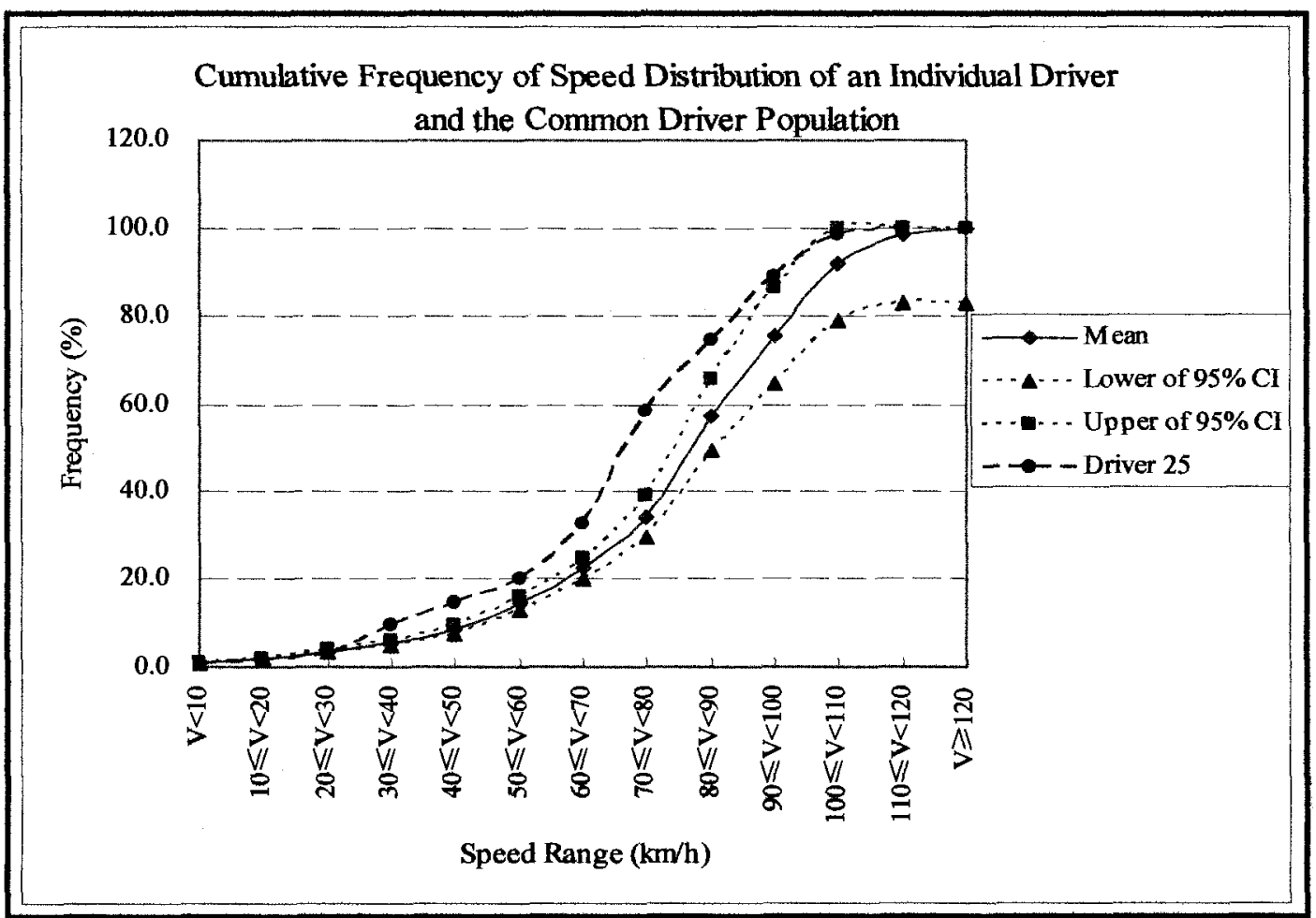

Figure B - 46: Cumulative Frequency of Speed Distribution of Driver 25 and the Common Driver Population. 


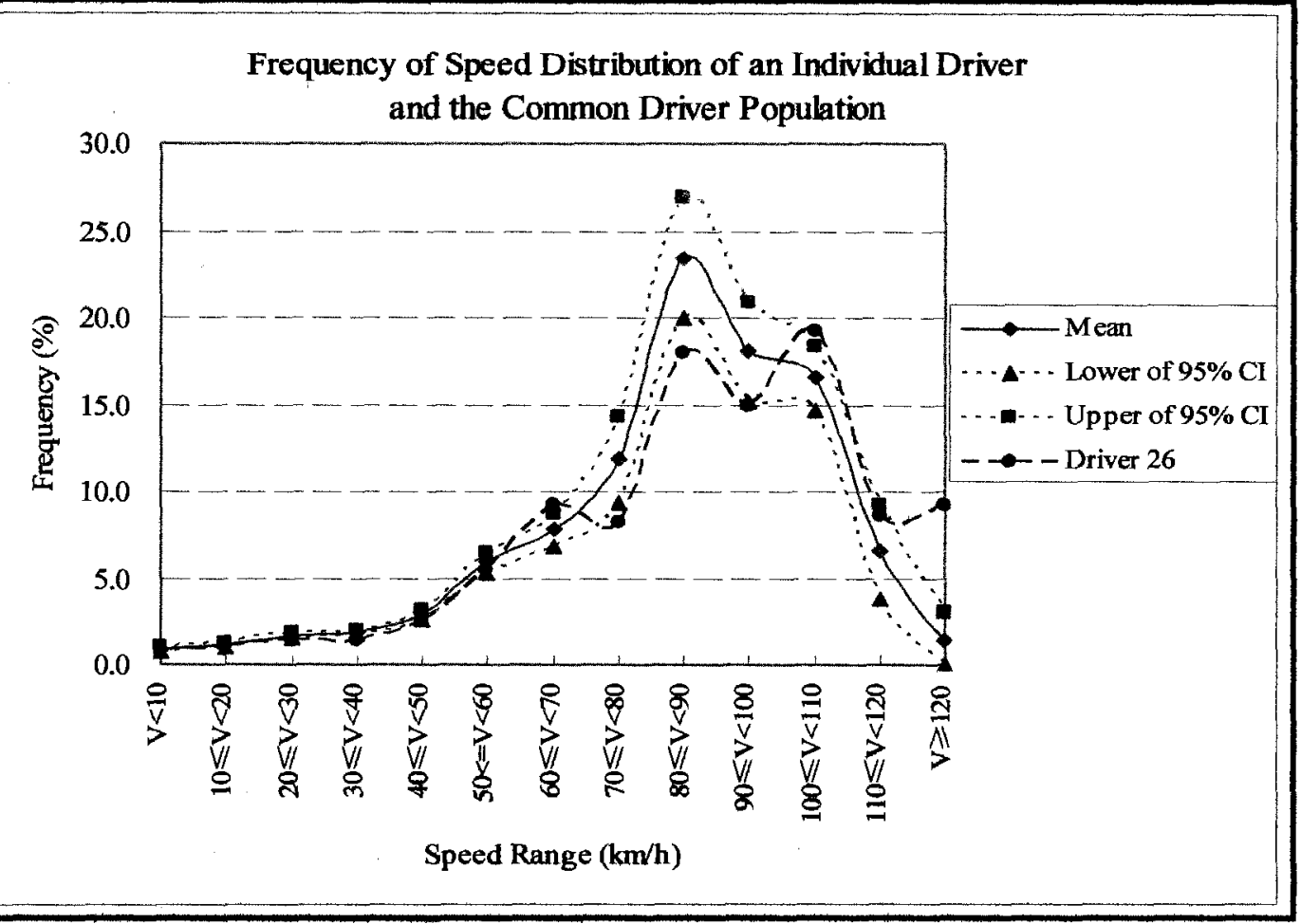

Figure B - 47: Speed Distribution of Driver 26 and the Common Driver Population.

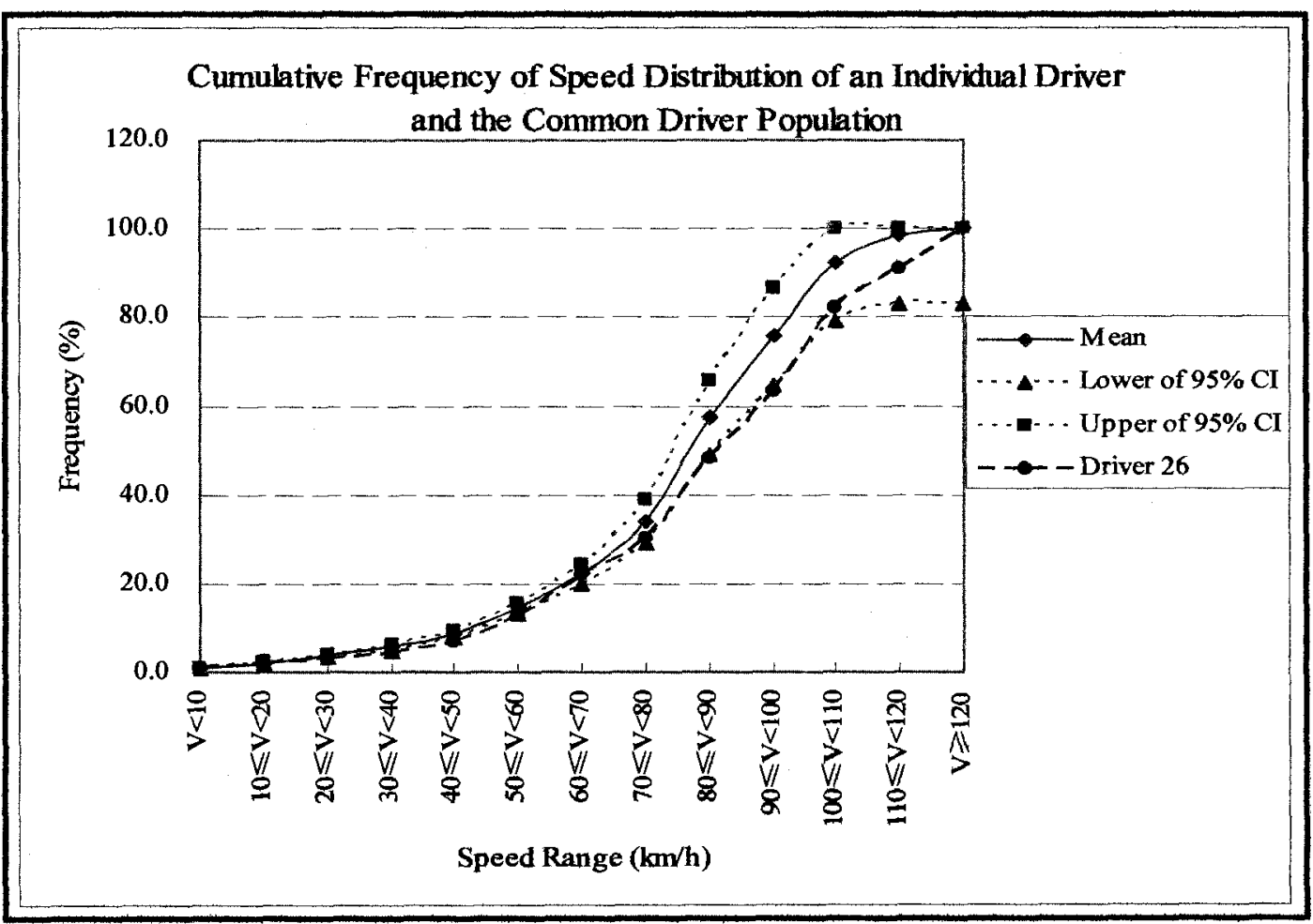

Figure B - 48: Cumulative Frequency of Speed Distribution of Driver 26 and the Common Driver Population. 


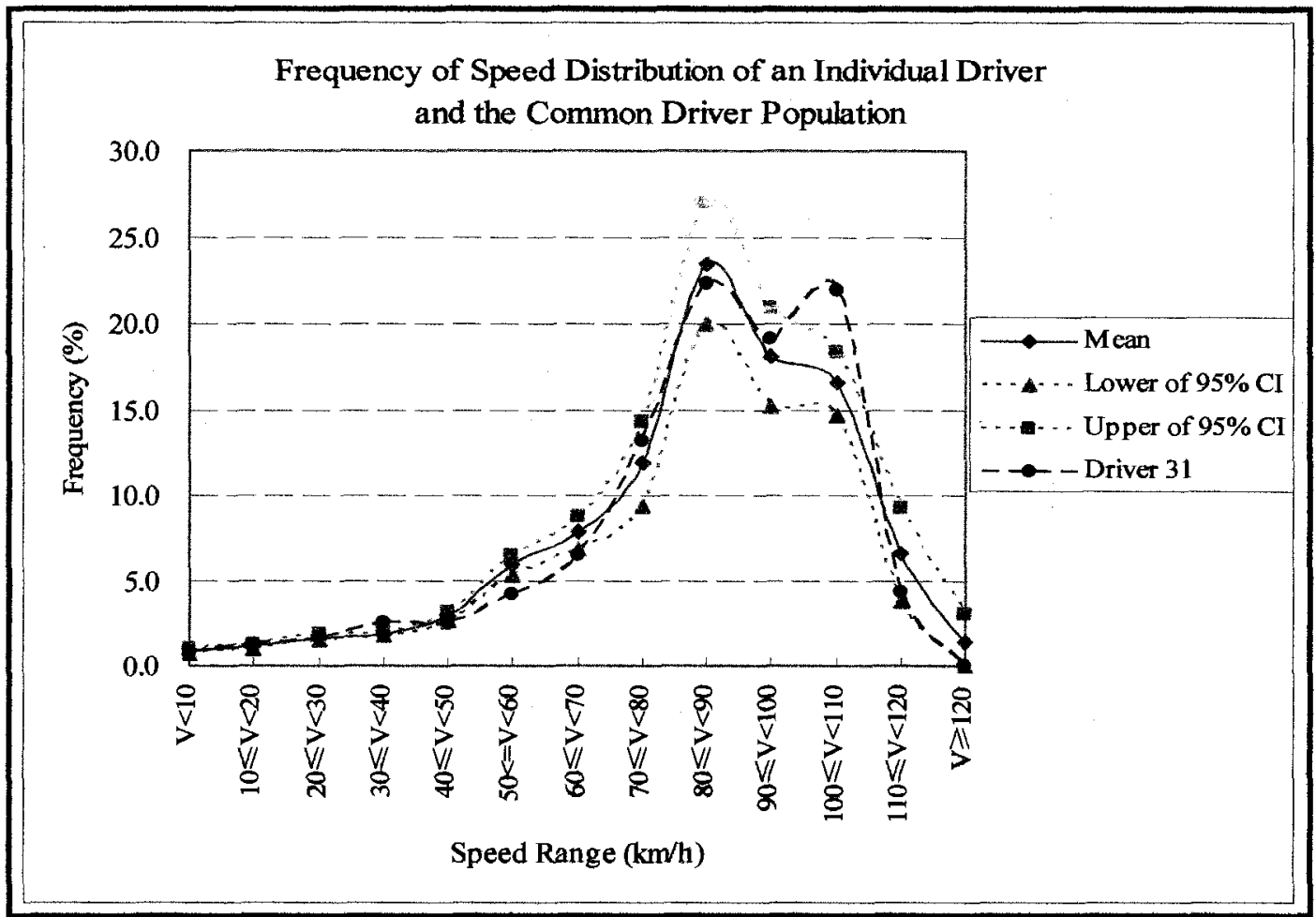

Figure B - 49: Speed Distribution of Driver 31 and the Common Driver Population.

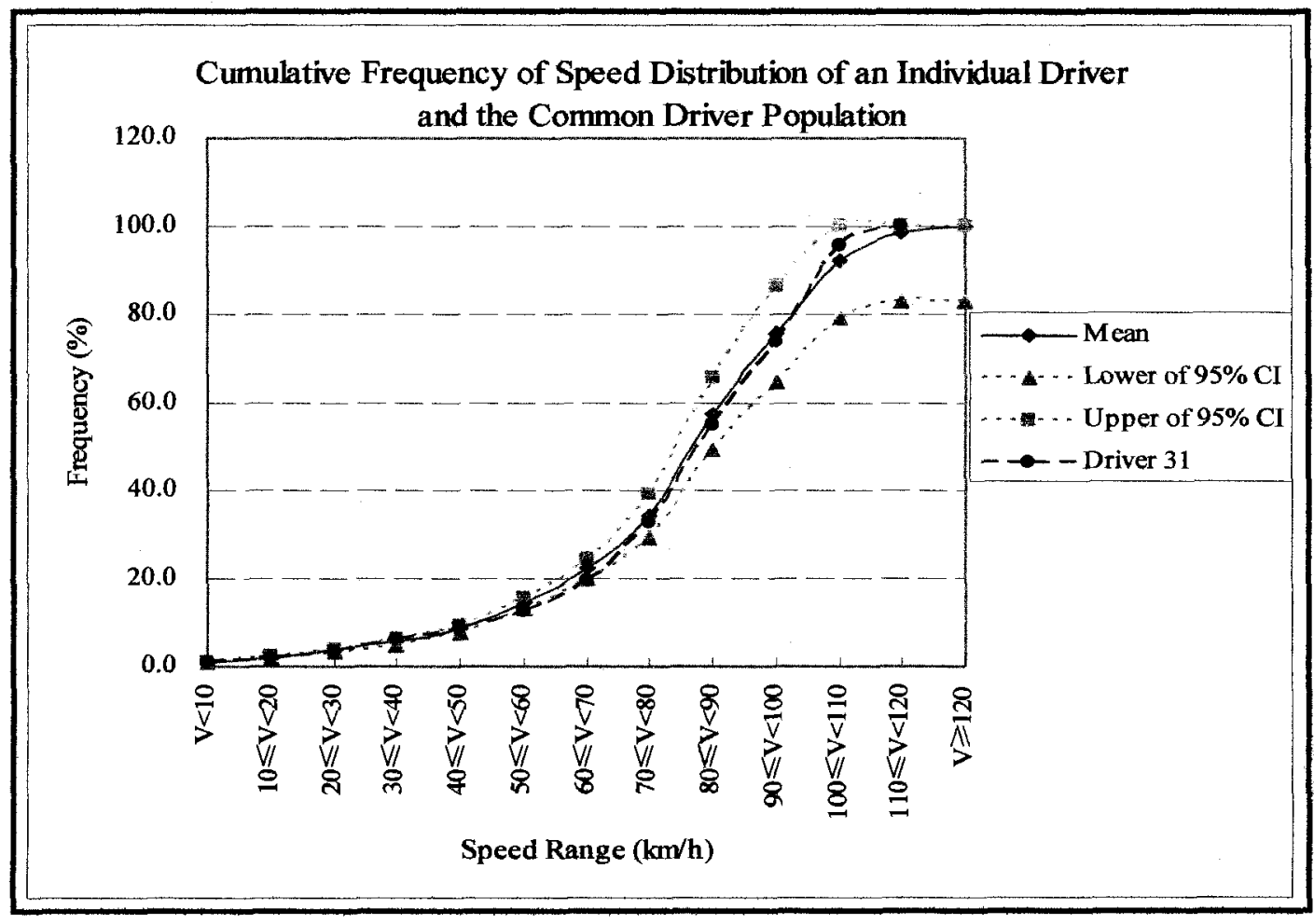

Figure B - 50: Cumulative Frequency of Speed Distribution of Driver 31 and the Common Driver Population. 


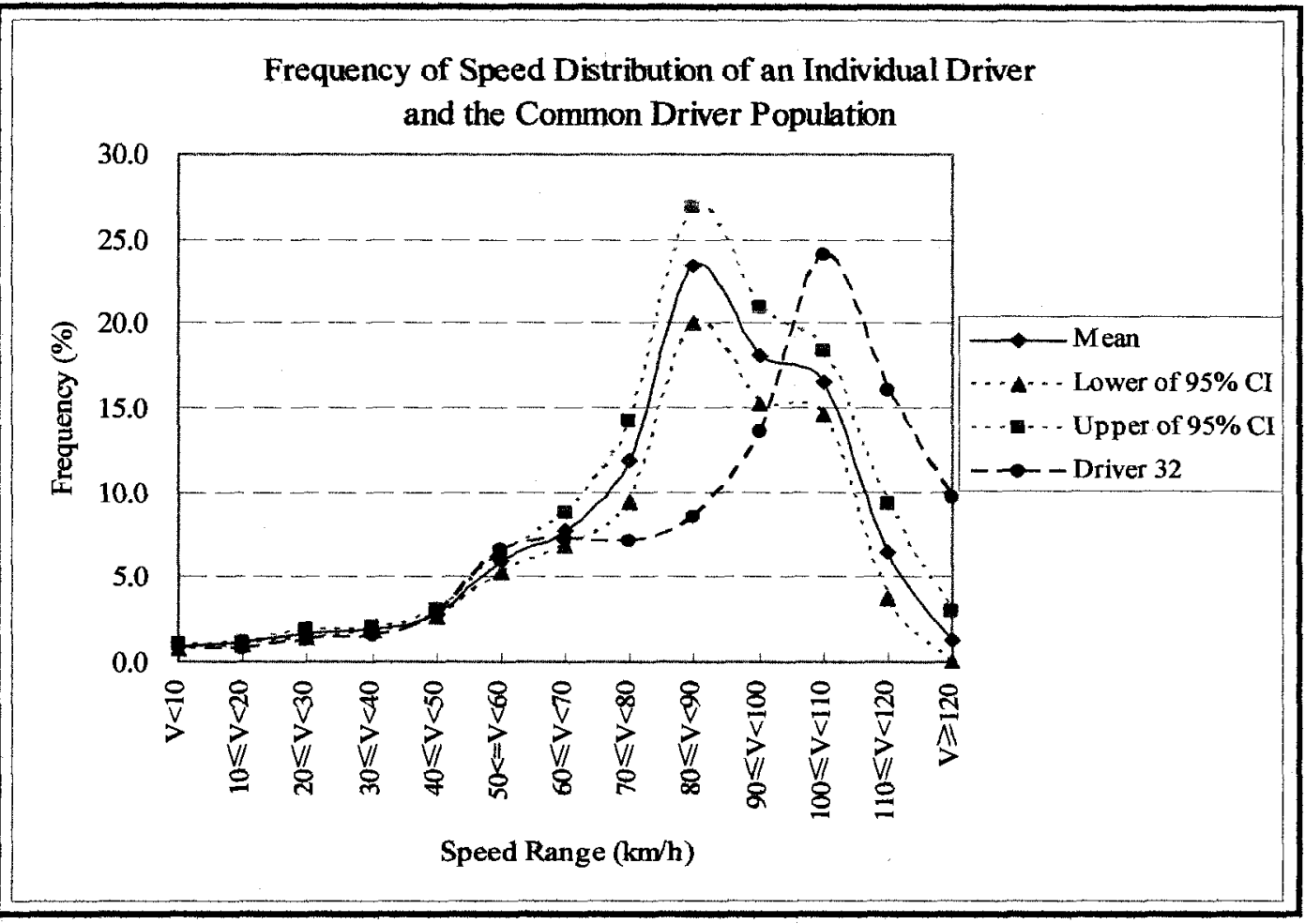

Figure B - 51: Speed Distribution of Driver 32 and the Common Driver Population.

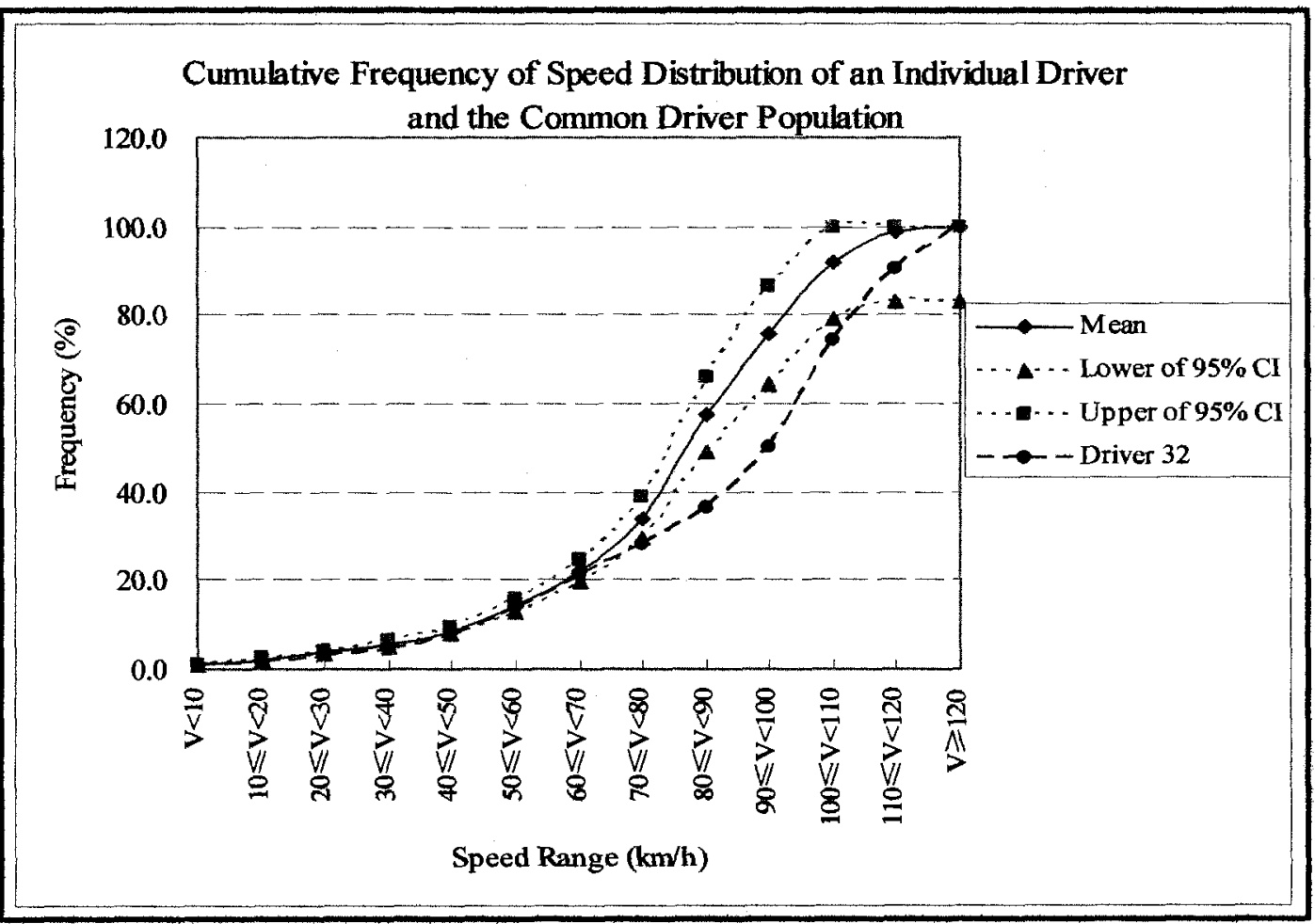

Figure B - 52: Cumulative Frequency of Speed Distribution of Driver 32 and the Common Driver Population. 


\section{APPENDIX C: ACCELERATION AND DECELERATION OF DIFFERENT TYPES OF DRIVERS}


Table C - 1: Statistics of Acceleration of the Three Driver Types under Free-Flow Conditions.

\begin{tabular}{|c|c|c|c|c|c|c|c|}
\hline \multirow{2}{*}{$\begin{array}{c}\text { Driver } \\
\text { Type }\end{array}$} & $\begin{array}{c}\text { Acceleration } \\
\text { Parameter } \\
\left(\mathrm{m} / \mathrm{s}^{2}\right)\end{array}$ & \multicolumn{6}{|c|}{ Speed Range $(\mathrm{km} / \mathrm{h})$} \\
\cline { 3 - 9 } & $\mathrm{V}<20$ & $20 \leq \mathrm{V}<40$ & $40 \leq \mathrm{V}<60$ & $60 \leq \mathrm{V}<80$ & $80 \leq \mathrm{V}<100$ & $\mathrm{~V} \geq 100$ \\
\hline \multirow{3}{*}{ Aggressive } & Mean & 1.322 & 1.235 & 0.907 & 0.488 & 0.316 & 0.210 \\
\cline { 2 - 9 } & $\mathrm{STD}$ & 0.633 & 0.547 & 0.499 & 0.331 & 0.238 & 0.155 \\
\hline \multirow{2}{*}{ Common } & Mean & 1.299 & 1.095 & 0.659 & 0.288 & 0.178 & 0.151 \\
\cline { 2 - 9 } & $\mathrm{STD}$ & 0.595 & 0.450 & 0.422 & 0.245 & 0.136 & 0.111 \\
\hline \multirow{2}{*}{ Defensive } & Mean & 1.260 & 1.115 & 0.513 & 0.222 & 0.165 & 0.166 \\
\cline { 2 - 9 } & $\mathrm{STD}$ & 0.622 & 0.541 & 0.400 & 0.180 & 0.127 & 0.113 \\
\hline
\end{tabular}

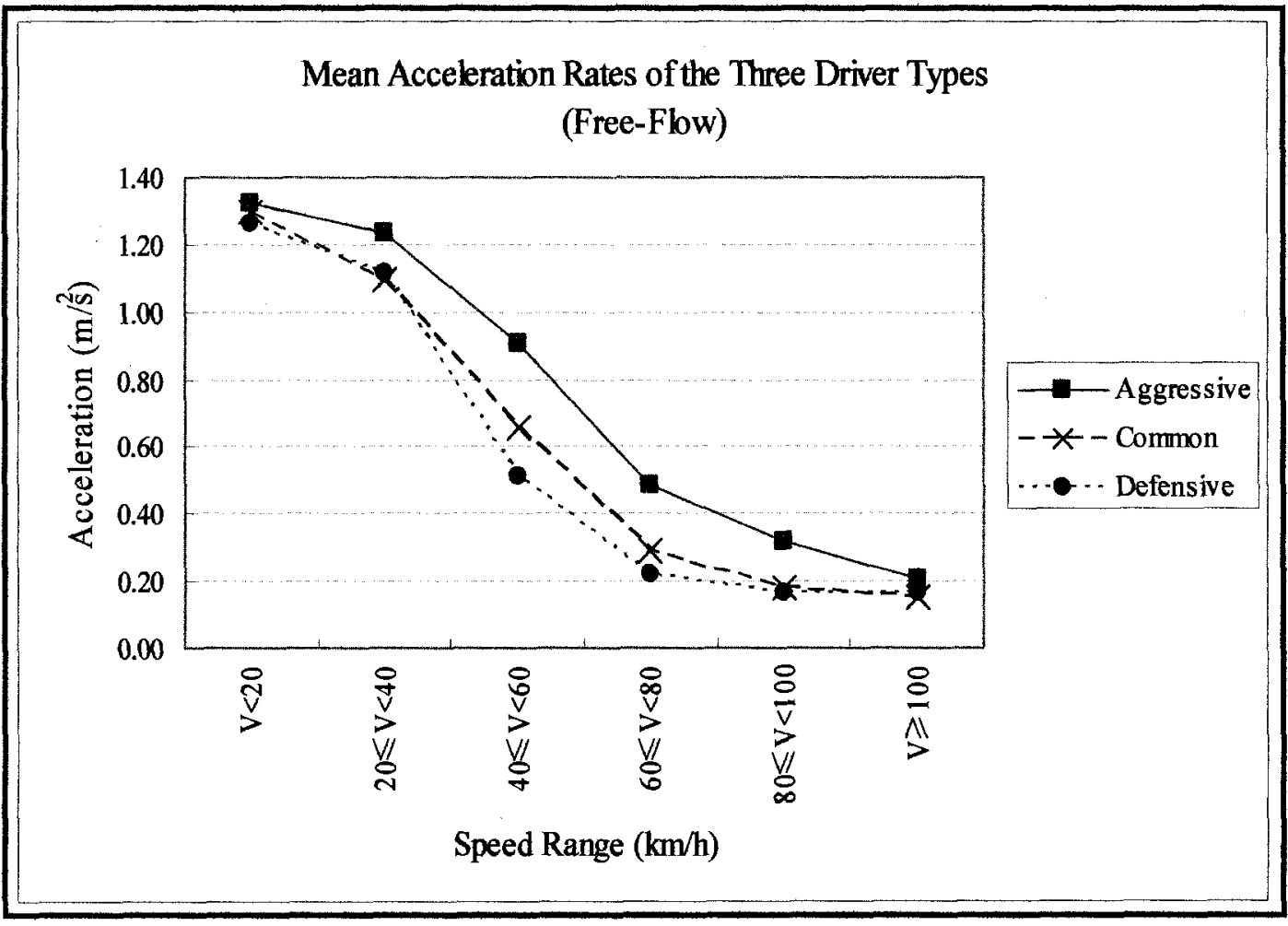

Figure C - 1: Acceleration of the Three Driver Types under Free-Flow Conditions. 
Table C - 2: Statistics of Acceleration of the Three Driver Types under Non-Free-Flow Conditions.

\begin{tabular}{|c|c|c|c|c|c|c|c|}
\hline \multirow{2}{*}{$\begin{array}{c}\text { Driver } \\
\text { Type }\end{array}$} & $\begin{array}{c}\text { Acceleration } \\
\text { Parameter } \\
\left(\mathrm{m} / \mathrm{s}^{2}\right)\end{array}$ & \multicolumn{6}{|c|}{ Speed Range $(\mathrm{km} / \mathrm{h})$} \\
\cline { 3 - 8 } & & $\mathrm{V}<20$ & $20 \leq \mathrm{V}<40$ & $40 \leq \mathrm{V}<60$ & $60 \leq \mathrm{V}<80$ & $80 \leq \mathrm{V}<100$ & $\mathrm{~V} \geq 100$ \\
\hline \multirow{2}{*}{ Aggressive } & Mean & 1.037 & 1.038 & 0.489 & 0.327 & 0.244 & 0.202 \\
\cline { 2 - 8 } & $\mathrm{STD}$ & 0.638 & 0.542 & 0.360 & 0.266 & 0.194 & 0.139 \\
\hline \multirow{2}{*}{ Common } & Mean & 1.049 & 0.727 & 0.395 & 0.259 & 0.200 & 0.148 \\
\cline { 2 - 8 } & $\mathrm{STD}$ & 0.616 & 0.444 & 0.306 & 0.196 & 0.155 & 0.108 \\
\hline \multirow{2}{*}{ Defensive } & Mean & 0.886 & 0.504 & 0.325 & 0.228 & 0.183 & 0.205 \\
\cline { 2 - 8 } & STD & 0.610 & 0.447 & 0.242 & 0.172 & 0.142 & 0.127 \\
\hline
\end{tabular}

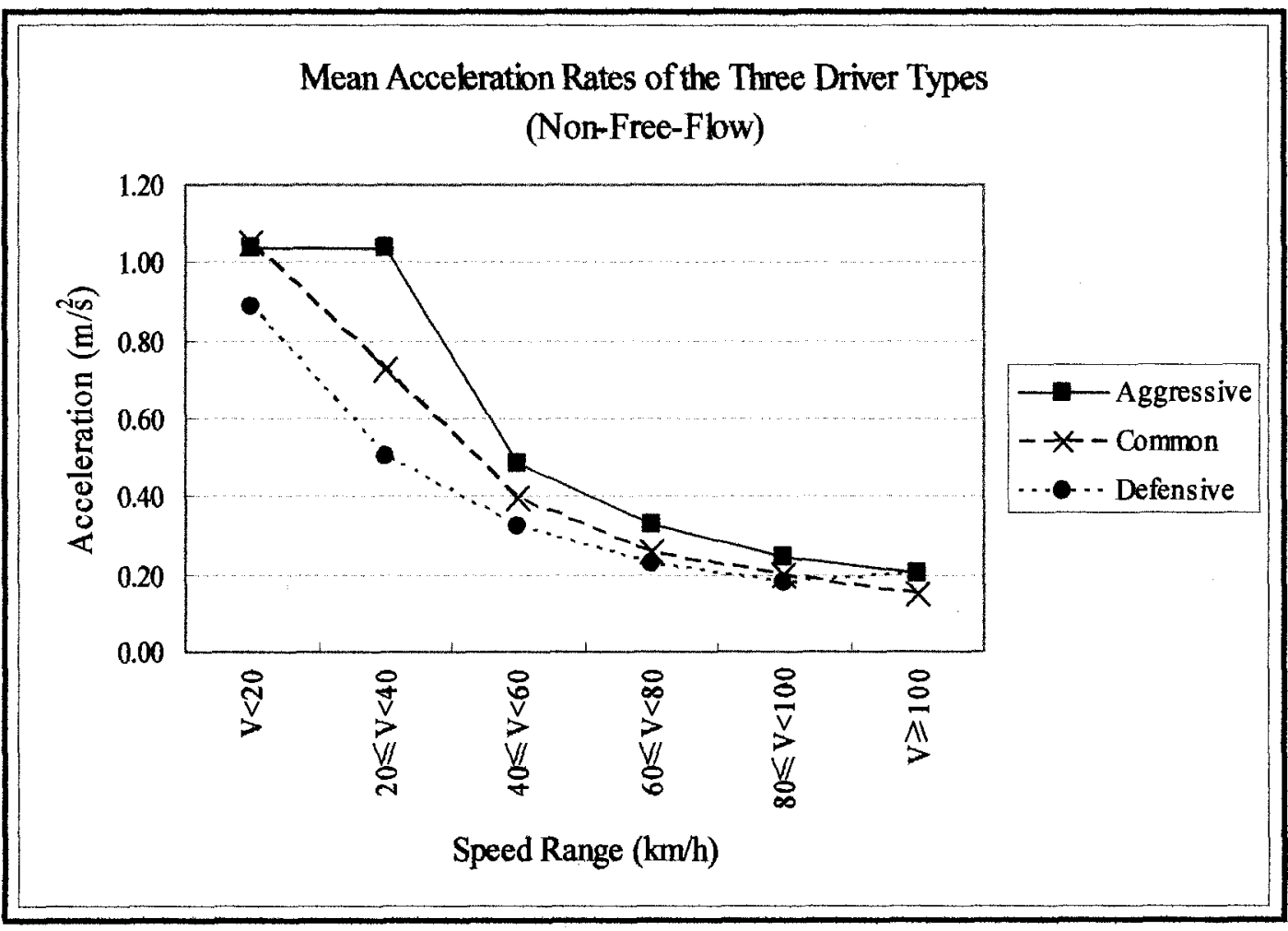

Figure C - 2: Acceleration of the Three Driver Types under Non-Free-Flow Conditions. 
Table C - 3: Statistics of Deceleration of the Three Driver Types under Free-Flow Conditions.

\begin{tabular}{|c|c|c|c|c|c|c|c|}
\hline \multirow{2}{*}{$\begin{array}{c}\text { Driver } \\
\text { Type }\end{array}$} & $\begin{array}{c}\text { Deceleration } \\
\text { Parameter } \\
\left(\mathrm{m} / \mathrm{s}^{2}\right)\end{array}$ & \multicolumn{6}{|c|}{ Speed Range $(\mathrm{km} / \mathrm{h})$} \\
\cline { 3 - 8 } & $\mathrm{V}<20$ & $20 \leq \mathrm{V}<40$ & $40 \leq \mathrm{V}<60$ & $60 \leq \mathrm{V}<80$ & $80 \leq \mathrm{V}<100$ & $\mathrm{~V} \geq 100$ \\
\hline \multirow{3}{*}{ Aggressive } & Mean & 0.855 & 1.692 & 1.113 & 0.513 & 0.359 & 0.248 \\
\cline { 2 - 8 } & $\mathrm{STD}$ & 0.690 & 0.852 & 0.874 & 0.567 & 0.353 & 0.248 \\
\hline \multirow{3}{*}{ Common } & Mean & 1.021 & 1.488 & 0.694 & 0.292 & 0.171 & 0.156 \\
\cline { 2 - 8 } & $\mathrm{STD}$ & 0.684 & 0.779 & 0.671 & 0.335 & 0.147 & 0.144 \\
\hline \multirow{3}{*}{ Defensive } & Mean & 0.998 & 1.280 & 0.485 & 0.212 & 0.171 & 0.188 \\
\cline { 2 - 8 } & $\mathrm{STD}$ & 0.619 & 0.706 & 0.528 & 0.200 & 0.126 & 0.159 \\
\hline
\end{tabular}

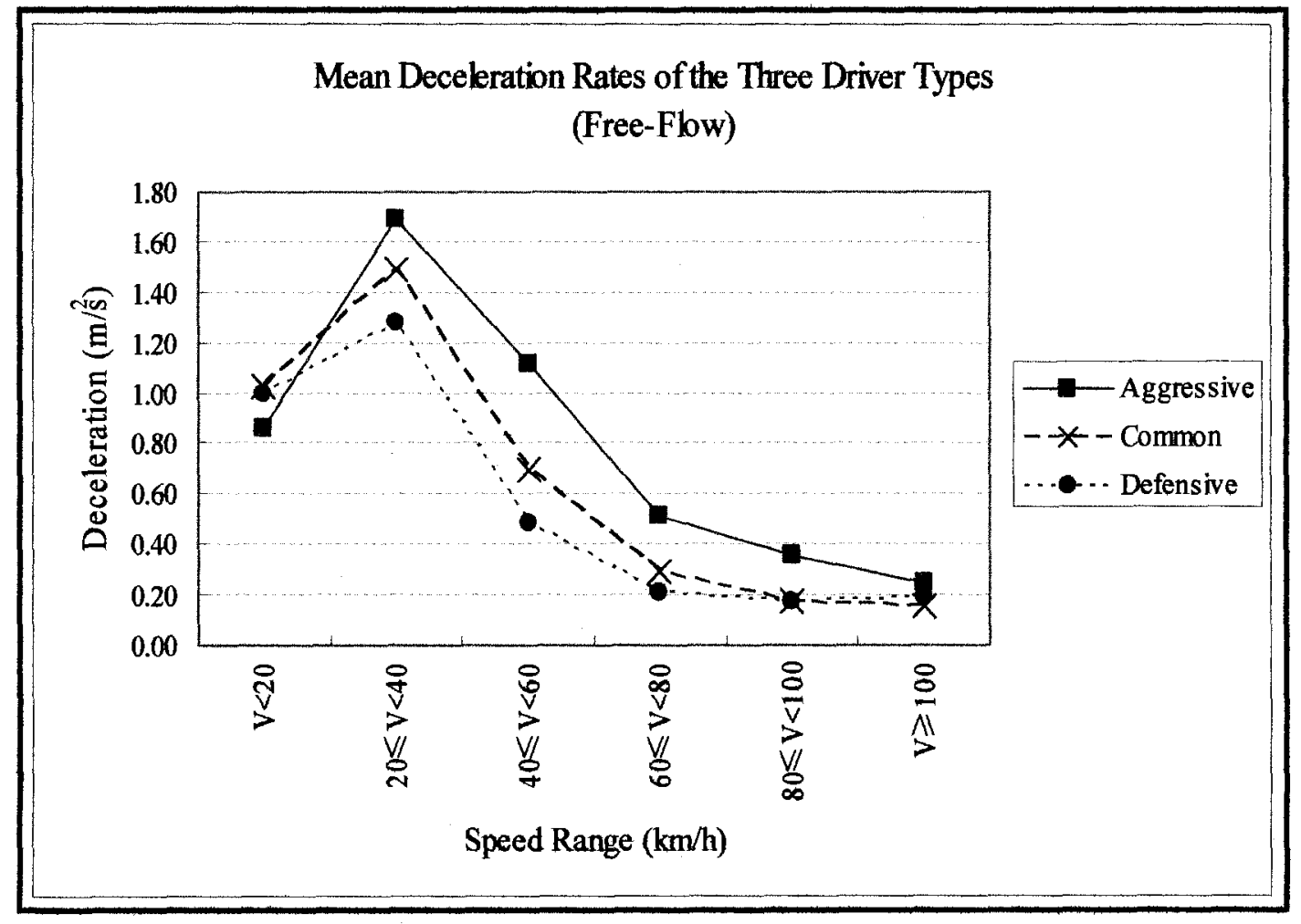

Figure C - 3: Deceleration of the Three Driver Types under Free-Flow Conditions. 
Table C - 4: Statistics of Deceleration of the Three Driver Types under Non-Free-Flow Conditions.

\begin{tabular}{|c|c|c|c|c|c|c|c|}
\hline \multirow{2}{*}{$\begin{array}{c}\text { Driver } \\
\text { Type }\end{array}$} & $\begin{array}{c}\text { Deceleration } \\
\text { Parameter } \\
\left(\mathrm{m} / \mathrm{s}^{2}\right)\end{array}$ & \multicolumn{6}{|c|}{ Speed Range $(\mathrm{km} / \mathrm{h})$} \\
\cline { 3 - 8 } & $\mathrm{V}<20$ & $20 \leq \mathrm{V}<40$ & $40 \leq \mathrm{V}<60$ & $60 \leq \mathrm{V}<80$ & $80 \leq \mathrm{V}<100$ & $\mathrm{~V} \geq 100$ \\
\hline \multirow{2}{*}{ Aggressive } & Mean & 1.086 & 1.220 & 0.573 & 0.393 & 0.272 & 0.248 \\
\cline { 2 - 8 } & $\mathrm{STD}$ & 0.751 & 0.769 & 0.592 & 0.394 & 0.259 & 0.235 \\
\hline \multirow{3}{*}{ Common } & Mean & 0.802 & 0.994 & 0.548 & 0.308 & 0.197 & 0.164 \\
\cline { 2 - 8 } & $\mathrm{STD}$ & 0.527 & 0.681 & 0.559 & 0.306 & 0.165 & 0.136 \\
\hline \multirow{2}{*}{ Defensive } & Mean & 1.071 & 0.676 & 0.425 & 0.239 & 0.193 & 0.196 \\
\cline { 2 - 8 } & $\mathrm{STD}$ & 0.673 & 0.731 & 0.475 & 0.216 & 0.153 & 0.158 \\
\hline
\end{tabular}

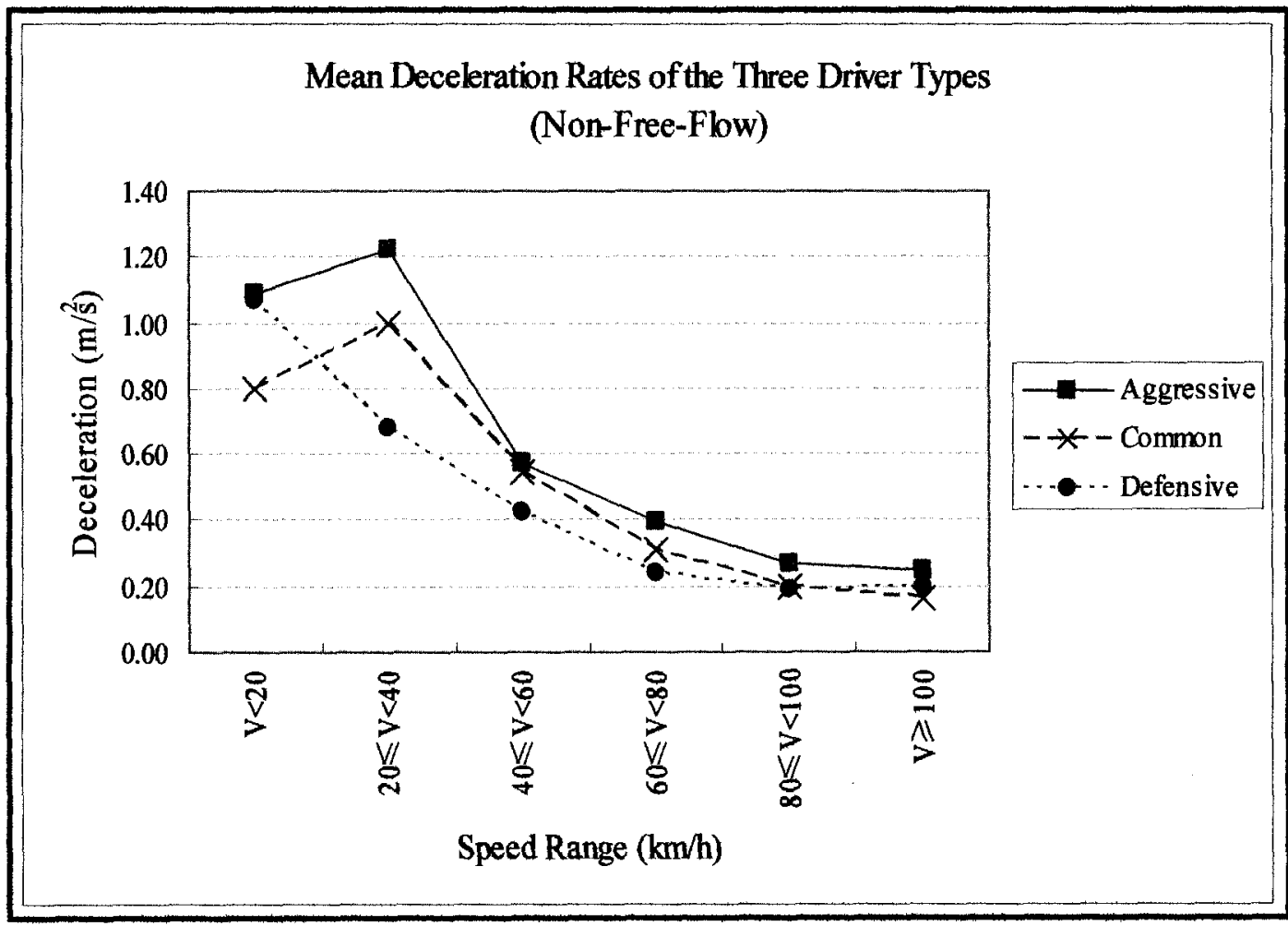

Figure C - 4: Deceleration of the Three Driver Types under Non-Free-Flow Conditions. 